Supporting Information for:

\title{
Silver-Assisted, Iridium-Catalyzed Allylation of Bis[(pinacolato)boryl]methane Allows the Synthesis of Enantioenriched Homoallylic Organoboronic Esters.
}

Miao Zhan ${ }^{*}$ Ren-zhe Lit, Ze-dong Mou* Chao-guo Cao ${ }^{*}$, Jie Liu*, Yuan-wei Chen*, Dawen Niu*

Department of Emergency, State Key Laboratory of Biotherapy and Cancer Center, West China Hospital, Sichuan University and Collaborative Innovation Center for Biotherapy, Chengdu 610041, China

E-mail:niudawen@scu.edu.cn; ywchen@scu.edu.cn; liujie2011@scu.edu.cn.

\section{Table of Contents}

$\begin{array}{lr}\text { 1. General Information } & \text { S2 }\end{array}$

$\begin{array}{lr}\text { 2. Figure S1 } & \text { S3 }\end{array}$

$\begin{array}{lr}\text { 3. Characterization Data of Substrates } & \text { S7 }\end{array}$

4. Ir-catalyzed Allylation of Bis[(pinacolato)boryl]methane (Table 2) S8-19

5. Derivatization of Homoallylic Boronates (Scheme 2)

$\begin{array}{ll}\text { 6. HPLC Data } & \text { S23 }\end{array}$

$\begin{array}{lr}\text { 7. NMR Spectra } & \text { S47 }\end{array}$

$\begin{array}{lr}\text { 8. References } & \text { S109 }\end{array}$ 


\section{General Information}

Flash column chromatography was performed using silica gel from Qindao Haiyang. Anhydrous solvents [tetrahydrofuran (THF), dichloromethane (DCM), and 1,4-dioxane] were purchased from Adamas, Energy Chemicals, or $\mathrm{J} \& \mathrm{~K}$, and used as received. $[\operatorname{Ir}(\mathrm{COD}) \mathrm{Cl}]_{2}$ was purchased from Adamas and used as received. Commercial $\mathrm{Ag}_{3} \mathrm{PO}_{4}$ (Energy Chemicals), AgOMs (Adamas), and AgOTf (Aldrich) were used as received. Chiral ligands ${ }^{1}$ and allylic carbonates used in this work were prepared according to literature procedures.

Bis[(pinacolato)boryl]methane were prepared according to literature procedure ${ }^{2}$ or purchased from TCI and used as received. NMR yields were determined by using 2-methyl naphthalene as an internal standard. All reported yields of the Ir/Ag-catalyzed allylation reactions are isolated yields following chromatography.

\section{General Analytical Information}

All new compounds were characterized by NMR spectroscopy, IR spectroscopy, high-resolution mass spectroscopy, and melting point (if solids). NMR spectra were recorded on a Bruker AMX 400 spectrometer and were calibrated using TMS or residual deuterated solvent as an internal reference $\left(\mathrm{CDCl}_{3}: 7.26 \mathrm{ppm}\right.$ for ${ }^{1} \mathrm{H}$ NMR and $77.16 \mathrm{ppm}$ for ${ }^{13} \mathrm{C}$ NMR). All IR spectra were taken on a Thermo Scientific Nicolet iS5 spectrometer (iD5 ATR, diamond). HRMS spectra were recorded on a Shimadzu LCMS-IT-TOF. Melting points (M.p.) were recorded on an INESA SGW X-4 melting point apparatus. GC/MS analyses were performed on an Agilent 6890/5977 instrument. The enantiomeric excesses (ee) of the products were determined by high-performance liquid chromatography (HPLC) analysis performed on Agilent 1100 Series or Waters 2695 Series chromatographs using a Diacel ${ }^{\circledR}$ chiral column $(25 \mathrm{~cm})$, or by ultraperformance convergence chromatography $\left(\mathrm{UPC}^{2}\right)$ on Waters Acquity UPC ${ }^{2}$ using Chiralpak IC-3 $(10 \mathrm{~cm})$. Optical rotations were measured on an INESA SGW-1 polarimeter with $[\alpha]_{\mathrm{D}}$ values reported in degrees; concentration (c) is in $\mathrm{g} / 100 \mathrm{~mL}$. 


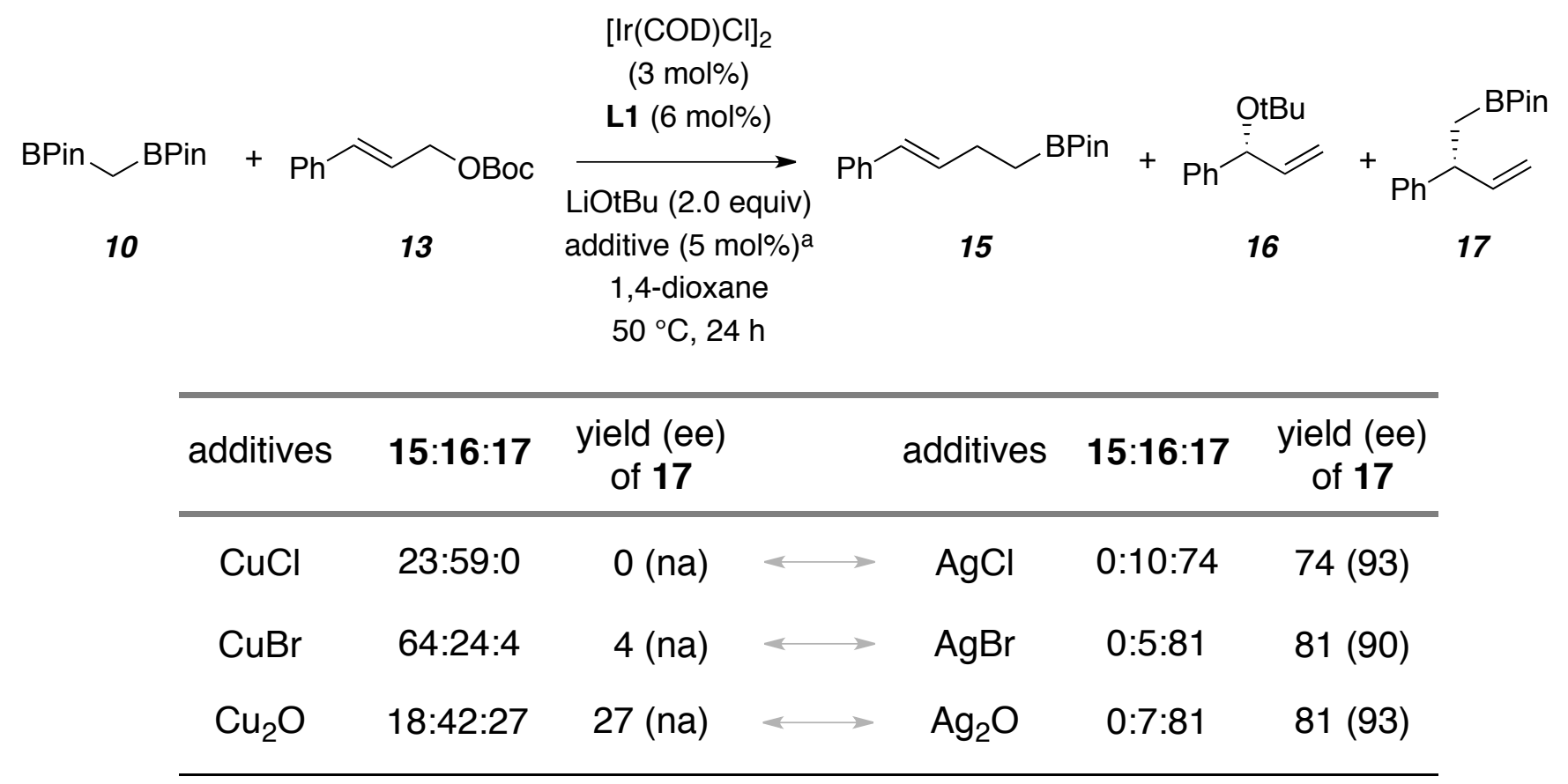

Figure S1. Comparison of Three Pairs of Silver and Copper Salts in the Ir-Catalyzed Allylation Reactions of Bisborylated Methane. 


\section{General Procedure for Ir-catalyzed Allylation of Bis[(pinacolato)boryl]methane}

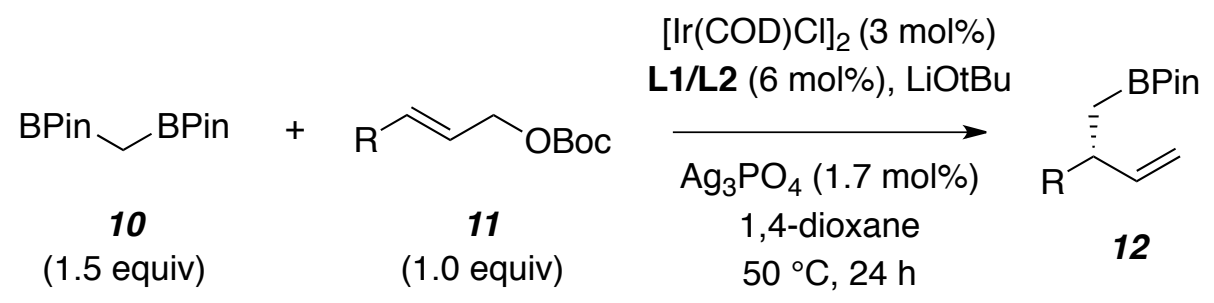

\section{Procedure A:}

$[\operatorname{Ir}(\mathrm{COD}) \mathrm{Cl}]_{2}(60 \mathrm{mg}, 0.090 \mathrm{mmol})$ and $\mathbf{L 1}(97 \mathrm{mg}, 0.18 \mathrm{mmol})$ were dissolved in anhydrous THF $(0.5 \mathrm{~mL})$ and propylamine $(0.5 \mathrm{~mL})$. The solution was heated at $50{ }^{\circ} \mathrm{C}$ for $20 \mathrm{~min}$, after which all volatiles were removed. The resulting yellow solid was then diluted in anhydrous 1,4-dioxane $(6.0 \mathrm{~mL})$ to get a stock solution.

In a nitrogen-filled glove-box, to a screw-capped vial containing $\mathrm{Ag}_{3} \mathrm{PO}_{4}(2.1 \mathrm{mg}, 0.0050 \mathrm{mmol}, 1.7 \mathrm{~mol} \%)$, bisborylmethane (10, $0.12 \mathrm{~g}, 0.45 \mathrm{mmol}, 1.5$ equiv), $\mathrm{LiO}^{t} \mathrm{Bu}(36 \mathrm{mg}, 0.45 \mathrm{mmol}, 1.5$ equiv), linear allylic tertbutyl carbonate 11 (if solid, $0.30 \mathrm{mmol}, 1.0$ equiv), and a stir bar were added a stock solution of the catalyst prepared in the above step $(0.6 \mathrm{~mL}, 0.009 \mathrm{mmol}, 3 \mathrm{~mol} \%)$. The vial was sealed with a cap containing a PTFE septum and removed from the glove box. Linear allylic tert-butyl carbonate, if liquid, was added via a syringe. The reaction was stirred at $50{ }^{\circ} \mathrm{C}$ for $24 \mathrm{~h}$. EtOAc $(10 \mathrm{~mL})$ was then added. The mixture was then washed with saturated $\mathrm{NaHCO}_{3}(5 \mathrm{~mL})$ and brine $(5 \mathrm{~mL})$. The organic phase was then dried $\left(\mathrm{Na}_{2} \mathrm{SO}_{4}\right)$, filtered, and concentrated under reduced pressure. Pure product was obtained after flash column chromatography.

\section{Procedure B:}

In a nitrogen-filled glove-box, anhydrous 1,4-dioxane $(0.60 \mathrm{~mL})$ was added to a screw-capped vial containing $[\mathrm{Ir}(\mathrm{COD}) \mathrm{Cl}]_{2}(6.0 \mathrm{mg}, 0.0090 \mathrm{mmol}, 3.0 \mathrm{~mol} \%)$ and $\mathbf{L 2}(0.018 \mathrm{mmol}, 6.0 \mathrm{~mol} \%), \mathrm{Ag}_{3} \mathrm{PO}_{4}(2.1 \mathrm{mg}, 0.0050 \mathrm{mmol}$, $1.7 \mathrm{~mol} \%$ ), bisborylmethane (10, $0.12 \mathrm{~g}, 0.45 \mathrm{mmol}, 1.5$ equiv), $\mathrm{LiO}^{t} \mathrm{Bu}$ (36 mg, $0.45 \mathrm{mmol}, 1.5$ equiv), linear allylic tert-butyl carbonate 11 (if solid, $0.30 \mathrm{mmol}, 1.0$ equiv), and a stir bar. The vial was sealed with a cap containing a PTFE septum and removed from the glove box. Linear allylic tert-butyl carbonate, if liquid, was added via a syringe. The reaction was stirred at $50{ }^{\circ} \mathrm{C}$ for $24 \mathrm{~h}$. EtOAc $(10 \mathrm{~mL})$ was then added. The mixture was then washed with saturated $\mathrm{NaHCO}_{3}(5 \mathrm{~mL})$ and brine $(5 \mathrm{~mL})$. The organic phase was then dried $\left(\mathrm{Na}_{2} \mathrm{SO}_{4}\right)$, filtered, and concentrated under reduced pressure. Pure product was obtained after flash column chromatography. 


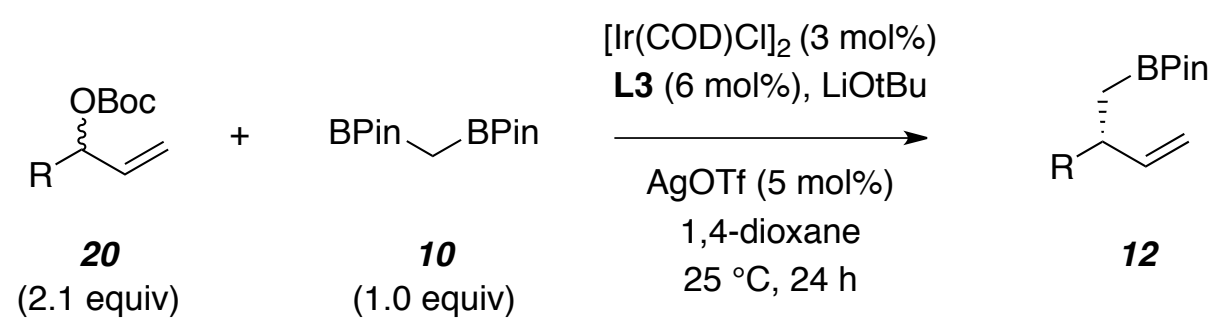

\section{Procedure C:}

In a nitrogen-filled glove-box, $[\operatorname{Ir}(\mathrm{COD}) \mathrm{Cl}]_{2}(6.0 \mathrm{mg}, 0.0090 \mathrm{mmol}, 3.0 \mathrm{~mol} \%)$ and $\mathbf{L 3}(18 \mathrm{mg}, 0.036 \mathrm{mmol}, 12$ mol\%) were added to a screw-capped vial equipped with a stir bar. Anhydrous 1,4-dioxane $(0.60 \mathrm{~mL})$ was added. The mixture was stirred for $20 \mathrm{~min}$ at room temperature before AgOTf (3.9 mg, $0.015 \mathrm{mmol}, 5.0 \mathrm{~mol} \%$ ), bisborylmethane ( $80 \mathrm{mg}, 0.30 \mathrm{mmol}, 1.0$ equiv), LiOtBu (36 mg, $0.45 \mathrm{mmol}, 1.5$ equiv) and branched allylic tertbutyl carbonate 20 ( $0.63 \mathrm{mmol}, 2.1$ equiv) were added. The vial was then sealed with a cap containing a PTFE septum and removed from the glove box. The reaction was stirred at $25^{\circ} \mathrm{C}$ for $24 \mathrm{~h}$. EtOAc $(10 \mathrm{~mL})$ was then added. The resulting mixture was washed with saturated $\mathrm{NaHCO}_{3}(5 \mathrm{~mL})$ and brine $(5 \mathrm{~mL})$. The organic phase was then dried $\left(\mathrm{Na}_{2} \mathrm{SO}_{4}\right)$, filtered, and concentrated under reduced pressure. Pure product was obtained after flash column chromatography. 


\section{Characterization Data for Allyl Carbonates.}<smiles>C=CC(OC(=O)OC(C)(C)C)c1ccc(Cl)c(Cl)c1</smiles>

tert-Butyl 1-(3,4-dichlorophenyl)allyl carbonate. IR (thin film, $\mathbf{~ c m}^{-\mathbf{1}}$ ) 3085, 1644, 1740, 1592, 1160, $850 ;{ }^{\mathbf{1}} \mathbf{H}$ NMR (400 MHz, $\left.\mathbf{C D C l}_{3}\right)$ d: $7.46(\mathrm{~d}, J=2.0 \mathrm{~Hz}, 1 \mathrm{H}), 7.43(\mathrm{~d}, J=8.3 \mathrm{~Hz}, 1 \mathrm{H}), 7.20(\mathrm{dd}, J=8.3,2.0 \mathrm{~Hz}, 1 \mathrm{H})$, 6.01-5.90 (m, 2H), 5.38-5.25 (m, 2H), 1.48 (s, 9H); ${ }^{13} \mathbf{C}$ NMR (101 MHz, $\mathbf{C D C l}_{3}$ ) d: 152.61, 139.16, 135.41, 132.87, 132.43, 130.71, 129.16, 126.48, 118.28, 83.01, 77.87, and 27.90; HRMS (DART-TOF) calculated for $\mathrm{C}_{14} \mathrm{H}_{16} \mathrm{Cl}_{2} \mathrm{NaO}_{3}[\mathrm{M}+\mathrm{Na}]^{+} \mathrm{m} / \mathrm{z} 325.0369$, found 325.0348.<smiles>C=CC(OC(=O)OC(C)(C)C)c1cccc(C#N)c1</smiles>

tert-Butyl 1-(3-cyanophenyl)allyl carbonate. IR (thin film, $\mathbf{c m}^{-1}$ ) 3079, 2233, 1746, 1641, 1584, 1144, $852 ;{ }^{1} \mathbf{H}$

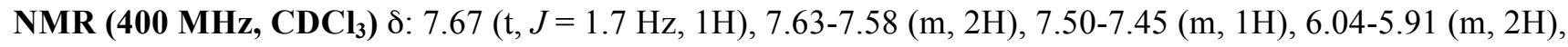

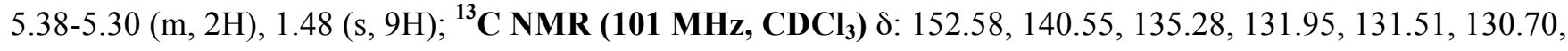
129.55, 118.66, 118.62, 112.95, 83.15, 78.01, and 27.89. HRMS (DART-TOF) calculated for $\mathrm{C}_{15} \mathrm{H}_{17} \mathrm{NNaO}_{3}$ $[\mathrm{M}+\mathrm{Na}]^{+} \mathrm{m} / \mathrm{z} 282.1104$, found 282.1101 .<smiles>Cn1ccc2cc(/C=C/COC(=O)OC(C)(C)C)ccc21</smiles>

(E)-tert-Butyl 3-(1-methyl-1H-indol-5-yl)allyl carbonate. IR (thin film, $\mathbf{c m}^{-1}$ ) 2984, 1737, 1616, 1513, 1158 , 857; ${ }^{1}$ H NMR (400 MHz, $\mathbf{C D C l}_{3}$ ) $\delta: 7.61$ (s, 1H), 7.34 (dd, J=8.6, 1.4 Hz, 1H), 7.26 (d, J=8.4 Hz, 1H), 7.03 $(\mathrm{d}, J=3.1 \mathrm{~Hz}, 1 \mathrm{H}), 6.79(\mathrm{~d}, J=15.8 \mathrm{~Hz}, 1 \mathrm{H}), 6.46(\mathrm{~d}, J=3.0 \mathrm{~Hz}, 1 \mathrm{H}), 6.25(\mathrm{dt}, J=15.7,6.8 \mathrm{~Hz}, 1 \mathrm{H}), 4.73(\mathrm{dd}, J$

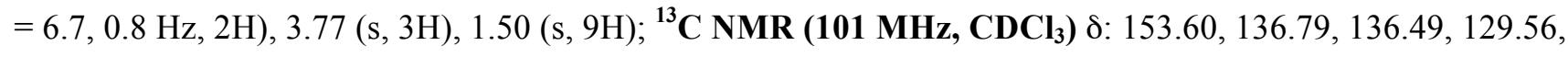
128.68, 127.87, 120.28, 120.12, 119.74, 109.49, 101.47, 82.18, 68.30, 33.05, and 27.94. HRMS (DART-TOF) calculated for $\mathrm{C}_{17} \mathrm{H}_{21} \mathrm{NNaO}_{3}[\mathrm{M}+\mathrm{Na}]^{+} \mathrm{m} / \mathrm{z} 310.1414$, found 310.1416 . 


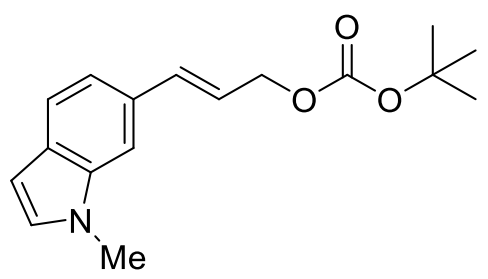

(E)-tert-butyl 3-(1-methyl-1H-indol-6-yl)allyl carbonate. IR (thin film, $\mathbf{c m}^{-\mathbf{1}}$ ) 2982, 1736, 1654, 1509, 1158, $856 ;{ }^{1}$ H NMR (400 MHz, CDCl $)$ ) $7.55(\mathrm{~d}, J=8.2 \mathrm{~Hz}, 1 \mathrm{H}), 7.30(\mathrm{~s}, 1 \mathrm{H}), 7.21(\mathrm{dd}, J=8.2,1.3 \mathrm{~Hz}, 1 \mathrm{H}), 7.04$ $(\mathrm{d}, J=3.1 \mathrm{~Hz}, 1 \mathrm{H}), 6.81(\mathrm{~d}, J=15.8 \mathrm{~Hz}, 1 \mathrm{H}), 6.44(\mathrm{dd}, \mathrm{J}=3.0,0.6 \mathrm{~Hz}, 1 \mathrm{H}), 6.32(\mathrm{dt}, J=15.8,6.7 \mathrm{~Hz}, 1 \mathrm{H}), 4.75$ $(\mathrm{dd}, J=6.7,1.0 \mathrm{~Hz}, 2 \mathrm{H}), 3.77$ (s, 3H), 1.51 (s, 9H); ${ }^{13} \mathbf{C} \mathbf{N M R}\left(\mathbf{1 0 1} \mathbf{M H z}, \mathbf{C D C l}_{3}\right) \delta: 153.50,136.88,136.26$, 129.98, 129.81, 128.64, 120.91, 120.61, 118.20, 108.04, 101.09, 82.15, 68.04, 32.87, and 27.85. HRMS (DARTTOF) calculated for $\mathrm{C}_{17} \mathrm{H}_{21} \mathrm{NNaO}_{3}[\mathrm{M}+\mathrm{Na}]^{+} \mathrm{m} / \mathrm{z} 310.1414$, found 310.1416 .<smiles>COc1ccc(/C=C/COC(=O)OC(C)(C)C)cn1</smiles>

(E)-tert-Butyl 3-(6-methoxypyridin-3-yl)allyl carbonate. IR (thin film, $\mathbf{c m}^{-\mathbf{1}}$ ) 2983, 1739, 1603, 1159, $844 ;{ }^{1} \mathbf{H}$ NMR (400 MHz, CDCl $)$ ) $8: 8.12(\mathrm{~d}, J=2.1 \mathrm{~Hz}, 1 \mathrm{H}), 7.67(\mathrm{dd}, J=8.7,2.3 \mathrm{~Hz}, 1 \mathrm{H}), 6.72(\mathrm{~d}, J=8.6 \mathrm{~Hz}, 1 \mathrm{H})$, $6.61(\mathrm{~d}, J=15.9 \mathrm{~Hz}, 1 \mathrm{H}), 6.19(\mathrm{dt}, J=15.9,6.5 \mathrm{~Hz}, 1 \mathrm{H}), 4.71(\mathrm{~d}, J=6.5 \mathrm{~Hz}, 2 \mathrm{H}), 3.94(\mathrm{~s}, 3 \mathrm{H}), 1.50(\mathrm{~s}, 9 \mathrm{H}) ;{ }^{13} \mathbf{C}$

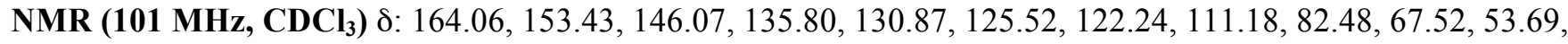
and 27.90; HRMS (DART-TOF) calculated for $\mathrm{C}_{14} \mathrm{H}_{19} \mathrm{NO}_{3}[\mathrm{M}+\mathrm{H}]^{+} \mathrm{m} / \mathrm{z} 266.1392$, found 266.1393. 


\section{Characterization Data for Compounds in Table 2.}

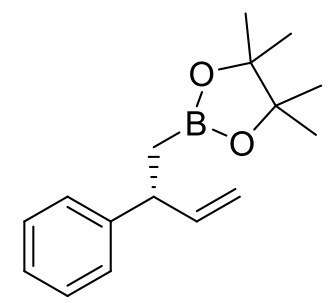

(R)-4,4,5,5-tetramethyl-2-(2-phenylbut-3-enyl)-1,3,2-dioxaborolane (17). Following the General Procedure A using tert-butyl cinnamyl carbonate ${ }^{3}\left(1.0 \mathrm{~g}, 4.3 \mathrm{mmol}, 1.0\right.$ equiv), $\mathrm{Ag}_{3} \mathrm{PO}_{4}(30 \mathrm{mg}, 0.071 \mathrm{mmol}, 1.7 \mathrm{~mol} \%)$, bisborylmethane (10, $1.7 \mathrm{~g}, 6.4 \mathrm{mmol}, 1.5$ equiv), $\mathrm{LiO}^{t} \mathrm{Bu}(0.51 \mathrm{~g}, 6.4 \mathrm{mmol}, 1.5$ equiv) and stock solution of iridium complex $(8.5 \mathrm{~mL}, 0.13 \mathrm{mmol})$. The product was isolated as a yellow oil $(1.0 \mathrm{~g}, 4.0 \mathrm{mmol}, 94 \%)$. IR (thin film, $\left.\mathbf{c m}^{-1}\right)$ 3062, 3028, 1638, 1601, 847; ${ }^{1} \mathbf{H}$ NMR (400 MHz, $\left.\mathbf{C D C l}_{3}\right)$ 8: 7.34-7.19 (m, 4H), 7.19-7.09 (m, 1H), 6.01 (ddd, $J=17.1,10.2,6.9 \mathrm{~Hz}, 1 \mathrm{H}), 5.03$ (dt, $J=17.1,1.5 \mathrm{~Hz}, 1 \mathrm{H}), 4.97$ (dt, $J=10.0,1.2 \mathrm{~Hz}, 1 \mathrm{H}), 3.61$ (ddd, $J$ $=7.7,7.7,7.7 \mathrm{~Hz}, 1 \mathrm{H}), 1.30(\mathrm{dd}, J=15.4,8.4 \mathrm{~Hz}, 1 \mathrm{H}), 1.24(\mathrm{dd}, J=15.4,8.0 \mathrm{~Hz}, 1 \mathrm{H})$, and $1.14(\mathrm{~s}, 12 \mathrm{H}) ;{ }^{13} \mathbf{C}$

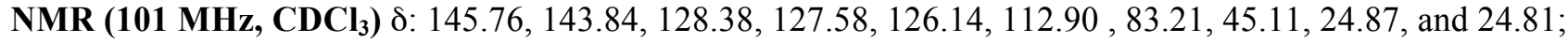
HRMS (DART-TOF) calculated for $\mathrm{C}_{16} \mathrm{H}_{23} \mathrm{BFO}_{2}{ }^{-}[\mathrm{M}+\mathrm{F}]^{-} \mathrm{m} / \mathrm{z}$ 277.1781, found 277.1774; HPLC analysis of the product after being oxidized to the corresponding alcohol by $\mathrm{NaBO}_{3} \cdot 4 \mathrm{H}_{2} \mathrm{O}(\mathrm{AD}-\mathrm{H}, \mathrm{Hexanes}: i \mathrm{PrOH}=99.6: 0.4,1$ $\mathrm{mL} / \mathrm{min})$ indicated $93 \%$ ee: $\mathrm{t}_{\mathrm{R}}($ major $)=45.4 \mathrm{~min}, \mathrm{t}_{\mathrm{R}}($ minor $)=39.2 \mathrm{~min} ;[\alpha]_{\mathbf{D}}{ }^{13}=50.8\left(\mathrm{c}=0.1, \mathrm{CHCl}_{3}\right)$.

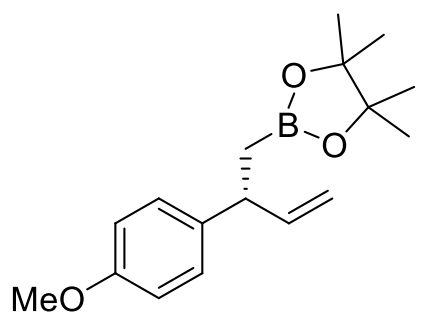

(R)-2-(2-(4-methoxyphenyl)but-3-enyl)-4,4,5,5-tetramethyl-1,3,2-dioxaborolane (12a). Following the

General Procedure A using (E)-tert-butyl 3-(4-methoxyphenyl)allyl carbonate ${ }^{3}$ (79 mg, 0.30 mmol, 1.0 equiv), $\mathrm{Ag}_{3} \mathrm{PO}_{4}$ (2.1 mg, $0.0050 \mathrm{mmol}, 1.7 \mathrm{~mol} \%$ ), bisborylmethane (10, $0.12 \mathrm{~g}, 0.45 \mathrm{mmol}, 1.5$ equiv), LiO ${ }^{t} \mathrm{Bu}(36 \mathrm{mg}$, $0.45 \mathrm{mmol}, 1.5$ equiv) and stock solution of iridium catalyst $(0.6 \mathrm{~mL}, 0.009 \mathrm{mmol})$. The product was isolated as a yellow oil (61 mg, $0.21 \mathrm{mmol}, 71 \%)$. IR (thin film, $\mathbf{~ c m}^{-1}$ ) 3078, 1636, 1511, 1145, 847; ${ }^{1} \mathrm{H} \mathrm{NMR} \mathrm{(400} \mathrm{MHz,}$ $\left.\mathbf{C D C l}_{3}\right) \delta: 7.14(\mathrm{~d}, J=8.5 \mathrm{~Hz}, 2 \mathrm{H}), 6.82(\mathrm{~d}, J=8.7 \mathrm{~Hz}, 2 \mathrm{H}), 5.98(\mathrm{ddd}, J=17.1,10.2,6.8 \mathrm{~Hz}, 1 \mathrm{H}), 5.01(\mathrm{dt}, J=$ $17.2,1.5 \mathrm{~Hz}, 1 \mathrm{H}), 4.94(\mathrm{dt}, J=10.4,1.4 \mathrm{~Hz}, 1 \mathrm{H}), 3.77(\mathrm{~s}, 3 \mathrm{H}), 3.56(\mathrm{ddd}, J=8.0,8.0,8.0 \mathrm{~Hz}, 1 \mathrm{H}), 1.27(\mathrm{dd}, J=$ 15.3, 8.2 Hz, 1H), $1.21(\mathrm{dd}, J=15.3,7.9 \mathrm{~Hz}, 1 \mathrm{H})$., and $1.15(\mathrm{~s}, 12 \mathrm{H}) ;{ }^{13} \mathbf{C} \mathbf{N M R}\left(\mathbf{1 0 1} \mathbf{M H z}, \mathbf{C D C l}_{3}\right) \delta: 158.01$, 144.20, 137.96, 128.49, 113.78, 112.59, 83.22, 55.40, 44.26, 24.90, and 24.86; HRMS (DART-TOF) calculated for $\mathrm{C}_{17} \mathrm{H}_{25} \mathrm{BO}_{3}[\mathrm{M}+\mathrm{H}]^{+} \mathrm{m} / \mathrm{z} 289.1975$, found 289.1985. HPLC analysis of the product after being oxidized to the 
corresponding alcohol by $\mathrm{NaBO}_{3} \cdot 4 \mathrm{H}_{2} \mathrm{O}(\mathrm{AD}-\mathrm{H}$, Hexanes: $i \mathrm{PrOH}=95: 5,1 \mathrm{~mL} / \mathrm{min})$ indicated $93 \%$ ee: $t_{\mathrm{R}}($ major $)$ $=12.2 \min , \mathrm{t}_{\mathrm{R}}($ minor $)=14.2 \mathrm{~min} ;[\boldsymbol{\alpha}]_{\mathbf{D}}{ }^{13}=51.8\left(\mathrm{c}=0.1, \mathrm{CHCl}_{3}\right)$.

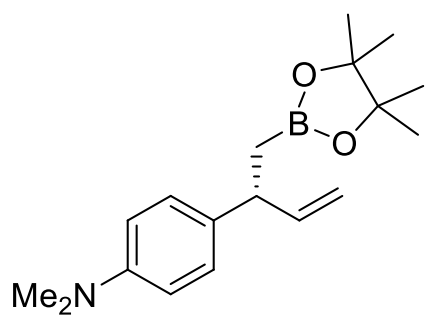

(R)-N,N-dimethyl-4-(1-(4,4,5,5-tetramethyl-1,3,2-dioxaborolan-2-yl)but-3-en-2-yl)aniline (12b). Following the General Procedure A using (E)-tert-butyl 3-(4-(dimethylamino)phenyl)allyl carbonate (83 mg, $0.30 \mathrm{mmol}$, 1.0 equiv), $\mathrm{Ag}_{3} \mathrm{PO}_{4}$ (2.1 mg, $0.0050 \mathrm{mmol}, 1.7 \mathrm{~mol} \%$ ), bisborylmethane (10, $0.12 \mathrm{~g}, 0.45 \mathrm{mmol}, 1.5$ equiv), $\mathrm{LiO}^{t} \mathrm{Bu}(36 \mathrm{mg}, 0.45 \mathrm{mmol}, 1.5$ equiv) and stock solution of iridium catalyst $(0.6 \mathrm{~mL}, 0.009 \mathrm{mmol})$. The reaction was stirred at $25{ }^{\circ} \mathrm{C}$ for $24 \mathrm{~h}$. The product was isolated as a yellow oil (71 mg, $\left.0.23 \mathrm{mmol}, 78 \%\right)$. IR (thin film, $\left.\mathbf{c m}^{-1}\right)$ 2978, 1614, 1520, 1141, and 846; ${ }^{1} \mathbf{H}$ NMR (400 MHz, $\left.\mathbf{C D C l}_{3}\right) \delta: 7.10$ (d, $\left.J=8.6 \mathrm{~Hz}, 2 \mathrm{H}\right), 6.68(\mathrm{~d}, J=8.7$ Hz, 2H), 5.98 (ddd, $J=17.1,10.2,6.9 \mathrm{~Hz}, 1 \mathrm{H}), 5.01$ (dt, $J=17.1,1.5 \mathrm{~Hz}, 1 \mathrm{H}), 4.92$ (dt, $J=10.4,1.6 \mathrm{~Hz}, 1 \mathrm{H})$, 3.53 (ddd, $J=7.6,7.6,7.6 \mathrm{~Hz}, 1 \mathrm{H}), 2.89$ (s, 6H), 1.27 (dd, $J=15.2,8.4 \mathrm{~Hz}, 1 \mathrm{H}), 1.21$ (dd, $J=15.2,7.6 \mathrm{~Hz}, 1 \mathrm{H})$. (m, 2H), 1.16 (s, 6H), 1.16 (s, 6H); ${ }^{13} \mathbf{C}$ NMR (101 MHz, CDCI $)$ ): 149.36, 144.45, 134.18, 128.07, 113.12, 112.23, 83.15, 44.14, 41.08, 24.94, and 24.87; HRMS (DART-TOF) calculated for $\mathrm{C}_{18} \mathrm{H}_{28} \mathrm{BO}_{2}[\mathrm{M}+\mathrm{H}]^{+} \mathrm{m} / \mathrm{z}$ 302.2291, found 302.2307. HPLC analysis of the product after being oxidized to the corresponding alcohol by $\mathrm{NaBO}_{3} \cdot 4 \mathrm{H}_{2} \mathrm{O}(\mathrm{AD}-\mathrm{H}$, Hexanes: $i \mathrm{PrOH}=95: 5,1 \mathrm{~mL} / \mathrm{min})$ indicated $93 \%$ ee: $\mathrm{t}_{\mathrm{R}}($ major $)=12.8 \mathrm{~min}, \mathrm{t}_{\mathrm{R}}($ minor $)=$ $15.3 \mathrm{~min} ;[\boldsymbol{\alpha}]_{\mathbf{D}}{ }^{13}=11.5\left(\mathrm{c}=0.2, \mathrm{CHCl}_{3}\right)$.

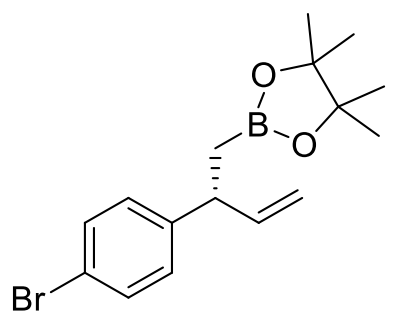

(R)-2-(2-(4-bromophenyl)but-3-enyl)-4,4,5,5-tetramethyl-1,3,2-dioxaborolane (12c). Following the General Procedure A using (E)-3-(4-bromophenyl)allyl tert-butyl carbonate 3 (94 mg, 0.30 mmol, 1.0 equiv), $\mathrm{Ag}_{3} \mathrm{PO}_{4}(3.5$ $\mathrm{mg}, 0.0083 \mathrm{mmol}, 2.8 \mathrm{~mol} \%$ ), bisborylmethane (10, $0.12 \mathrm{~g}, 0.45 \mathrm{mmol}, 1.5$ equiv), $\mathrm{LiO}^{t} \mathrm{Bu}$ (36 mg, $0.45 \mathrm{mmol}$, 1.5 equiv) and stock solution of the iridium catalyst $(0.6 \mathrm{~mL}, 0.025 \mathrm{M}, 0.015 \mathrm{mmol})$. The reaction was stirred at $50{ }^{\circ} \mathrm{C}$ for $36 \mathrm{~h}$. The product was isolated as a yellow oil ( $\left.81 \mathrm{mg}, 0.24 \mathrm{mmol}, 80 \%\right)$. IR (thin film, $\left.\mathbf{~ c m}^{-1}\right) 3078$, 1647, 1487, 1144, 847; ${ }^{1} \mathbf{H}$ NMR (400 MHz, $\mathbf{C D C l}_{3}$ ) $8: 7.39$ (d, $\left.J=8.4 \mathrm{~Hz}, 2 \mathrm{H}\right), 7.10$ (d, $\left.J=8.3 \mathrm{~Hz}, 2 \mathrm{H}\right), 5.95$ $(\mathrm{ddd}, J=17.0,10.2,6.8 \mathrm{~Hz}, 1 \mathrm{H}), 5.07-4.93(\mathrm{~m}, 2 \mathrm{H}), 3.57(\mathrm{ddd}, J=7.7,7.7,7.7 \mathrm{~Hz}, 1 \mathrm{H}), 1.28(\mathrm{dd}, J=15.3,8.1$ 


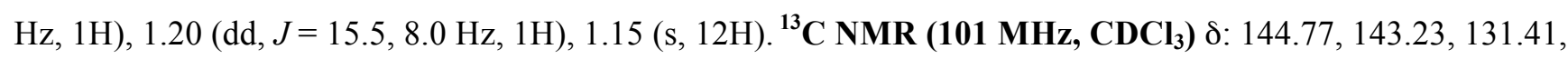
129.43, 119.85, 113.40, 83.34, 44.51, 24.87, and 24.85; HRMS (DART-TOF) calculated for $\mathrm{C}_{16} \mathrm{H}_{22} \mathrm{BBrFO}_{2}{ }^{-}$ $[\mathrm{M}+\mathrm{F}]^{-} \mathrm{m} / \mathrm{z}$ 355.0886, found 355.0899; HPLC analysis of the product after being oxidized to the corresponding alcohol by $\mathrm{NaBO}_{3} \cdot 4 \mathrm{H}_{2} \mathrm{O}(\mathrm{AD}-\mathrm{H}$, Hexanes: $i \mathrm{PrOH}=95: 5,1 \mathrm{~mL} / \mathrm{min})$ indicated $93 \%$ ee: $\mathrm{t}_{\mathrm{R}}$ (major) $=9.5 \mathrm{~min}, \mathrm{t}_{\mathrm{R}}$ $($ minor $)=11.1 \mathrm{~min} ;[\alpha]_{\mathbf{D}}{ }^{13}=36.6\left(\mathrm{c}=0.1, \mathrm{CHCl}_{3}\right)$.

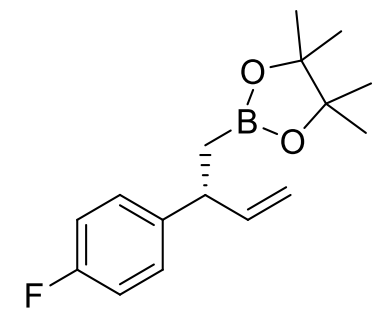

(R)-2-(2-(4-fluorophenyl)but-3-enyl)-4,4,5,5-tetramethyl-1,3,2-dioxaborolane (12d). Following the General Procedure A using (E)-tert-butyl 3-(4-fluorophenyl)allyl carbonate ${ }^{4}$ (76 mg, 0.30 mmol, 1.0 equiv), $\mathrm{Ag}_{3} \mathrm{PO}_{4}(2.1$ $\mathrm{mg}, 0.0050 \mathrm{mmol}, 1.7 \mathrm{~mol} \%$ ), bisborylmethane (10, $0.12 \mathrm{~g}, 0.45 \mathrm{mmol}, 1.5$ equiv), LiO ${ }^{t} \mathrm{Bu}(36 \mathrm{mg}, 0.45 \mathrm{mmol}$, 1.5 equiv) and stock solution of the iridium catalyst $(0.6 \mathrm{~mL}, 0.009 \mathrm{mmol})$. The product was isolated as a yellow oil (72 mg, 0.26 mmol, 87\%). IR (thin film, $\mathbf{c m}^{-1}$ ) 3079, 3038, 1637, 1509, 1145, 847; ${ }^{1}$ H NMR (400 MHz,

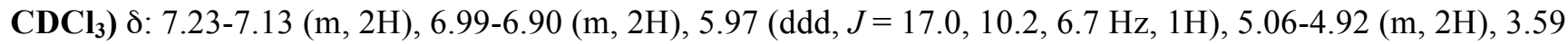
$(\mathrm{ddd}, J=7.7,7.7,7.7 \mathrm{~Hz}, 1 \mathrm{H}), 1.28(\mathrm{dd}, J=15.3,7.9 \mathrm{~Hz}, 1 \mathrm{H}), 1.21(\mathrm{dd}, J=15.4,8.2 \mathrm{~Hz}, 1 \mathrm{H}), 1.14(\mathrm{~s}, 12 \mathrm{H}) .{ }^{13} \mathbf{C}$ NMR (101 MHz, CDCl $)$ ) $\delta: 161.46(\mathrm{~d}, J=243.4 \mathrm{~Hz}), 143.75,141.37(\mathrm{~d}, J=3.1 \mathrm{~Hz}), 129.02(\mathrm{~d}, J=7.8 \mathrm{~Hz})$, $115.05(\mathrm{~d}, J=21.1 \mathrm{~Hz}), 113.03,83.29,44.33,24.86$, and 24.84; ${ }^{\mathbf{1 9}} \mathbf{F}$ NMR (376 MHz, CDCl $\left.\mathbf{3}\right) \delta:-117.6$;

HRMS (DART-TOF) calculated for $\mathrm{C}_{16} \mathrm{H}_{22} \mathrm{BF}_{2} \mathrm{O}_{2}{ }^{-}[\mathrm{M}+\mathrm{F}]^{-} \mathrm{m} / \mathrm{z}$ 295.1686, found 295.1683; HPLC analysis of the product after being oxidized to the corresponding alcohol by $\mathrm{NaBO}_{3} \cdot 4 \mathrm{H}_{2} \mathrm{O}(\mathrm{AD}-\mathrm{H}, \mathrm{Hexanes}: i \mathrm{PrOH}=99: 1,1$ $\mathrm{mL} / \mathrm{min})$ indicated $92 \%$ ee: $\mathrm{t}_{\mathrm{R}}($ major $)=19.2 \mathrm{~min}, \mathrm{t}_{\mathrm{R}}($ minor $)=21.3 \mathrm{~min} ;[\boldsymbol{\alpha}]_{\mathbf{D}}{ }^{\mathbf{1 3}}=30.8\left(\mathrm{c}=0.1, \mathrm{CHCl}_{3}\right)$.<smiles>C=C[C@H](CB1OC(C)(C)C(C)(C)O1)c1ccc(Cl)cc1</smiles>

(R)-2-(2-(4-chlorophenyl)but-3-enyl)-4,4,5,5-tetramethyl-1,3,2-dioxaborolane (12e). Following the General Procedure A using (E)-tert-butyl 3-(4-chlorophenyl)allyl carbonate ${ }^{5}$ (81 mg, 0.30 mmol, 1.0 equiv), $\mathrm{Ag}_{3} \mathrm{PO}_{4}(3.5$ $\mathrm{mg}, 0.0083 \mathrm{mmol}, 2.8 \mathrm{~mol} \%$ ), bisborylmethane (10, $0.12 \mathrm{~g}, 0.45 \mathrm{mmol}, 1.5$ equiv), $\mathrm{LiO}^{t} \mathrm{Bu}$ (36 mg, $0.45 \mathrm{mmol}$, 1.5 equiv) and stock solution $(0.6 \mathrm{~mL}, 0.015 \mathrm{mmol})$. The reaction was stirred at $50{ }^{\circ} \mathrm{C}$ for $36 \mathrm{~h}$. The product was

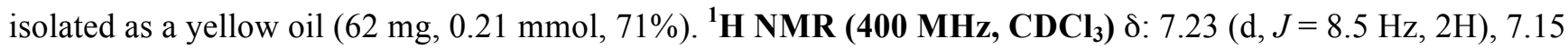


$(\mathrm{d}, J=8.5 \mathrm{~Hz}, 2 \mathrm{H}), 5.96(\mathrm{ddd}, J=17.0,10.2,6.8 \mathrm{~Hz}, 1 \mathrm{H}), 5.05-4.94(\mathrm{~m}, 2 \mathrm{H}), 3.58(\mathrm{ddd}, J=7.7,7.7,7.7 \mathrm{~Hz}, 1 \mathrm{H})$, $1.28(\mathrm{dd}, J=15.2,8.0 \mathrm{~Hz}, 1 \mathrm{H}), 1.20(\mathrm{dd}, J=14.7,7.3 \mathrm{~Hz}, 1 \mathrm{H}), 1.15(\mathrm{~s}, 12 \mathrm{H}) .{ }^{\mathbf{1 3}} \mathbf{C} \mathbf{N M R}\left(\mathbf{1 0 1} \mathbf{M H z}, \mathbf{C D C l}_{3}\right) \delta$ : 144.26, 143.36, 131.79, 129.02, 128.46, 113.34, 83.34, 44.46, 24.87, and 24.85; HRMS (DART-TOF) calculated for $\mathrm{C}_{16} \mathrm{H}_{22} \mathrm{BClFO}_{2}{ }^{-}[\mathrm{M}+\mathrm{F}]^{-} \mathrm{m} / \mathrm{z}$ 311.1391, found 311.1397; HPLC analysis of the product after being oxidized to the corresponding alcohol by $\mathrm{NaBO}_{3} \cdot 4 \mathrm{H}_{2} \mathrm{O}(\mathrm{AS}-\mathrm{H}$, Hexanes: $i \mathrm{PrOH}=95: 5,1 \mathrm{~mL} / \mathrm{min})$ indicated $92 \%$ ee: $\mathrm{t}_{\mathrm{R}}$ $($ major $)=9.5 \mathrm{~min}, \mathrm{t}_{\mathrm{R}}($ minor $)=8.8 \mathrm{~min} ;[\alpha]_{\mathbf{D}}{ }^{13}=69.7\left(\mathrm{c}=0.2, \mathrm{CHCl}_{3}\right)$.

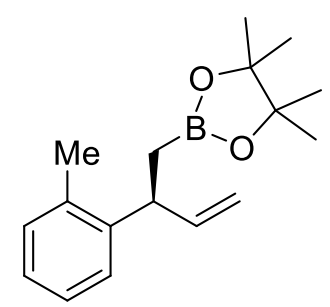

(S)-4,4,5,5-tetramethyl-2-(2-o-tolylbut-3-enyl)-1,3,2-dioxaborolane (12f). Following the General Procedure B using (E)-tert-butyl 3-o-tolylallyl carbonate ${ }^{4}\left(75 \mathrm{mg}, 0.30 \mathrm{mmol}, 1.0\right.$ equiv), $\mathrm{Ag}_{3} \mathrm{PO}_{4}(2.1 \mathrm{mg}, 0.0050 \mathrm{mmol}, 1.7$ mol\%), bisborylmethane (10, $0.12 \mathrm{~g}, 0.45 \mathrm{mmol}, 1.5$ equiv), $\mathrm{LiO}^{t} \mathrm{Bu}(36 \mathrm{mg}, 0.45 \mathrm{mmol}, 1.5 \mathrm{equiv}),[\operatorname{Ir}(\mathrm{COD}) \mathrm{Cl}]_{2}$ (6.0 mg, $0.0090 \mathrm{mmol}, 3.0 \mathrm{~mol} \%$ ) and $\mathbf{L} 2(8.3 \mathrm{mg}, 0.018 \mathrm{mmol}, 6.0 \mathrm{~mol} \%)$. The product was isolated as a yellow

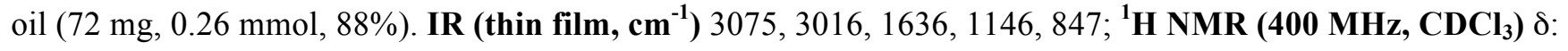
7.21-7.02 (m, 4H), $5.94(\mathrm{ddd}, J=17.0,10.5,6.6 \mathrm{~Hz}, 1 \mathrm{H}), 4.99-4.88(\mathrm{~m}, 1 \mathrm{H}), 3.84(\mathrm{ddd}, J=7.9,7.9,7.9 \mathrm{~Hz}, 1 \mathrm{H})$, $2.36(\mathrm{~s}, 3 \mathrm{H}), 1.30(\mathrm{dd}, J=12.2,5.0 \mathrm{~Hz}, 1 \mathrm{H}), 1.25(\mathrm{dd}, J=12.6,4.4 \mathrm{~Hz}, 1 \mathrm{H}), 1.11(\mathrm{~s}, 6 \mathrm{H}), 1.09(\mathrm{~s}, 6 \mathrm{H}) ;{ }^{13} \mathbf{C} \mathbf{N M R}$

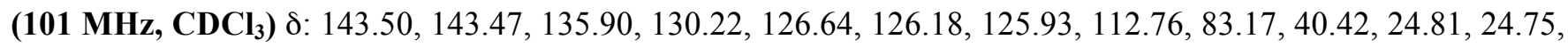
and 19.75; HRMS (DART-TOF) calculated for $\mathrm{C}_{17} \mathrm{H}_{25} \mathrm{BFO}_{2}{ }^{-}[\mathrm{M}+\mathrm{F}]^{-} \mathrm{m} / \mathrm{z}$ 291.1937, found 291.1946; HPLC analysis of the product after being oxidized to the corresponding alcohol by $\mathrm{NaBO}_{3} \cdot 4 \mathrm{H}_{2} \mathrm{O}(\mathrm{AD}-\mathrm{H}, \mathrm{Hexanes}$ : $i \operatorname{PrOH}=99.6: 0.4,0.3 \mathrm{~mL} / \mathrm{min})$ indicated $92 \%$ ee: $\mathrm{t}_{\mathrm{R}}($ major $)=108.3 \mathrm{~min}, \mathrm{t}_{\mathrm{R}}(\operatorname{minor})=133.4 \mathrm{~min} ;[\boldsymbol{\alpha}]_{\mathbf{D}}{ }^{\mathbf{1 3}}=$ $14.831\left(\mathrm{c}=0.2, \mathrm{CHCl}_{3}\right)$.

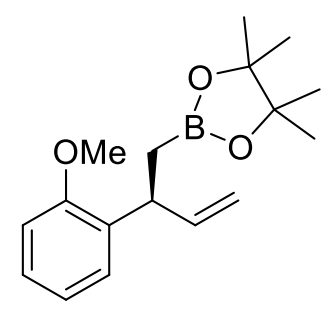

(S)-2-(2-(2-methoxyphenyl)but-3-enyl)-4,4,5,5-tetramethyl-1,3,2-dioxaborolane (12g). Following the General Procedure B using (E)-tert-butyl 3-(2-methoxyphenyl)allyl carbonate ${ }^{6}$ (79 mg, 0.30 mmol, 1.0 equiv), $\mathrm{Ag}_{3} \mathrm{PO}_{4}$ (2.1 mg, $0.0050 \mathrm{mmol}, 1.7 \mathrm{~mol} \%$ ), bisborylmethane (10, $0.12 \mathrm{~g}, 0.45 \mathrm{mmol}, 1.5$ equiv), $\mathrm{LiO}^{t} \mathrm{Bu}(36 \mathrm{mg}, 0.45$ mmol, 1.5 equiv), $[\operatorname{Ir}(\mathrm{COD}) \mathrm{Cl}]_{2}(6.0 \mathrm{mg}, 0.0090 \mathrm{mmol}, 3.0 \mathrm{~mol} \%)$ and $\mathbf{L 2}(8.3 \mathrm{mg}, 0.018 \mathrm{mmol}, 6.0 \mathrm{~mol} \%)$. The product was isolated as a yellow oil (74 $\mathrm{mg}, 0.26 \mathrm{mmol}, 85 \%)$. IR (thin film, $\mathbf{~ c m}^{-1}$ ) 3077, 1637, 1599, 1145, 847; 
${ }^{1}$ H NMR (400 MHz, CDCl $)$ ) $:$ 7.20-7.10 (m, 2H), 6.89 (td, $\left.J=7.5,0.9 \mathrm{~Hz}, 1 \mathrm{H}\right), 6.82(\mathrm{~d}, J=8.1 \mathrm{~Hz}, 1 \mathrm{H}), 6.05$ $(\mathrm{ddd}, J=17.0,10.2,6.7 \mathrm{~Hz}, 1 \mathrm{H}), 5.00(\mathrm{dt}, J=17.2,1.4 \mathrm{~Hz}, 1 \mathrm{H}), 4.93(\mathrm{dt}, J=10.0,1.2 \mathrm{~Hz}, 1 \mathrm{H}), 4.05(\mathrm{ddd}, J=$ 7.6, 7.6, 7.6 Hz, 1H), $3.81(\mathrm{~s}, 3 \mathrm{H}), 1.30(\mathrm{dd}, J=13.1,6.6 \mathrm{~Hz}, 1 \mathrm{H}), 1.25(\mathrm{dd}, J=13.1,5.5 \mathrm{~Hz}, 1 \mathrm{H}), 1.14(\mathrm{~s}, 6 \mathrm{H})$, 1.13 (s, 6H); ${ }^{13} \mathbf{C}$ NMR (101 MHz, $\mathbf{C D C l}_{3}$ ) $\delta: ~ 156.95,143.30,134.11,127.89,127.03,120.62,112.62,110.72$, 83.05, 55.55, 37.94, 24.86, and 24.77; HRMS (DART-TOF) calculated for $\mathrm{C}_{17} \mathrm{H}_{25} \mathrm{BO}_{3}[\mathrm{M}+\mathrm{H}]^{+} \mathrm{m} / \mathrm{z} 289.1975$, found 289.1973. HPLC analysis of the product after being oxidized to the corresponding alcohol by $\mathrm{NaBO}_{3} \cdot 4 \mathrm{H}_{2} \mathrm{O}$ (IA, Hexanes: $\left.i \mathrm{PrOH}=99.3: 0.7,1 \mathrm{~mL} / \mathrm{min}\right)$ indicated $86 \%$ ee: $\mathrm{t}_{\mathrm{R}}($ major $)=28.2 \mathrm{~min}, \mathrm{t}_{\mathrm{R}}($ minor $)=$ $30.2 \mathrm{~min} ;[\alpha]_{\mathbf{D}}{ }^{13}=-56.5\left(\mathrm{c}=0.1, \mathrm{CHCl}_{3}\right)$.

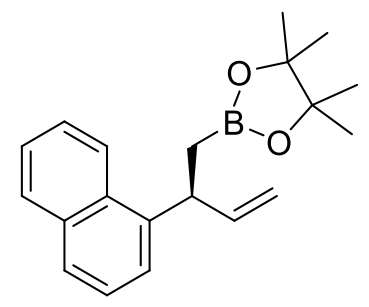

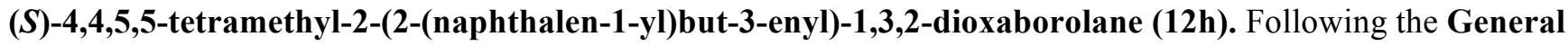
Procedure B using (E)-tert-butyl 3-(naphthalen-1-yl)allyl carbonate 5 (85 mg, $0.30 \mathrm{mmol}, 1.0$ equiv), $\mathrm{Ag}_{3} \mathrm{PO}_{4}(2.1$ $\mathrm{mg}, 0.0050 \mathrm{mmol}, 1.7 \mathrm{~mol} \%)$, bisborylmethane (10, $0.12 \mathrm{~g}, 0.45 \mathrm{mmol}, 1.5$ equiv), $\mathrm{LiO}^{t} \mathrm{Bu}$ (36 mg, $0.45 \mathrm{mmol}$, 1.5 equiv), $[\mathrm{Ir}(\mathrm{COD}) \mathrm{Cl}]_{2}(6.0 \mathrm{mg}, 0.0090 \mathrm{mmol}, 3.0 \mathrm{~mol} \%)$ and $\mathbf{L 2}(8.3 \mathrm{mg}, 0.018 \mathrm{mmol}, 6.0 \mathrm{~mol} \%)$. The product was isolated as a yellow oil (71 mg, $0.23 \mathrm{mmol}, 77 \%)$. IR (thin film, $\left.\mathbf{c m}^{-1}\right)$ 3048, 1636, 1597, 1145, 847; ${ }^{1} \mathbf{H}$ NMR (400 MHz, $\left.\mathbf{C D C l}_{3}\right) \delta: 8.21(\mathrm{~d}, J=8.4 \mathrm{~Hz}, 1 \mathrm{H}), 7.83(\mathrm{~d}, J=7.9 \mathrm{~Hz}, 1 \mathrm{H}), 7.69(\mathrm{dd}, J=6.7,2.6 \mathrm{~Hz}, 1 \mathrm{H})$, $7.52-7.39(\mathrm{~m}, 4 \mathrm{H}), 6.13(\mathrm{ddd}, J=17.0,10.3,6.4 \mathrm{~Hz}, 1 \mathrm{H}), 5.08(\mathrm{dt}, J=17.2,1.4 \mathrm{~Hz}, 1 \mathrm{H}), 5.03(\mathrm{dt}, J=10.3,1.3$ $\mathrm{Hz}, 1 \mathrm{H}), 4.47$ (ddd, $J=7.6,7.6,7.6 \mathrm{~Hz}, 1 \mathrm{H}), 1.50-1.39(\mathrm{~m}, 2 \mathrm{H}), 1.11(\mathrm{~s}, 6 \mathrm{H}), 1.07(\mathrm{~s}, 6 \mathrm{H}) ;{ }^{13} \mathbf{C} \mathbf{N M R}(\mathbf{1 0 1} \mathbf{M H z}$, $\left.\mathbf{C D C l}_{3}\right) \delta: 143.46,141.77,134.06,131.64,128.83,126.79,125.69,125.65,125.38,124.09,124.05,113.52,83.24$, 39.74, 24.85, and 24.74; HRMS (DART-TOF) calculated for $\mathrm{C}_{20} \mathrm{H}_{25} \mathrm{BFO}_{2}{ }^{-}[\mathrm{M}+\mathrm{F}]^{-} \mathrm{m} / \mathrm{z} 327.1937$, found 327.1940 ; HPLC analysis of the product after being oxidized to the corresponding alcohol by $\mathrm{NaBO}_{3} \cdot 4 \mathrm{H}_{2} \mathrm{O}(\mathrm{AD}-$ $\mathrm{H}$, Hexanes: $i \mathrm{PrOH}=95: 5,1 \mathrm{~mL} / \mathrm{min})$ indicated $90 \%$ ee: $\mathrm{t}_{\mathrm{R}}($ major $)=14.6 \mathrm{~min}, \mathrm{t}_{\mathrm{R}}(\operatorname{minor})=13.2 \mathrm{~min} ;[\boldsymbol{\alpha}]_{\mathbf{D}}{ }^{\mathbf{1 3}}=$ $28.3\left(\mathrm{c}=0.1, \mathrm{CHCl}_{3}\right)$. 


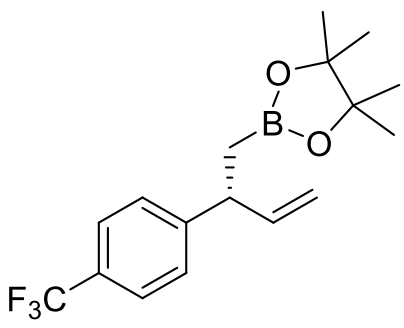

\section{(R)-4,4,5,5-tetramethyl-2-(2-(4-(trifluoromethyl)phenyl)but-3-enyl)-1,3,2-}

dioxaborolane (12i). Following the General Procedure C using tert-butyl 1-(4-

(trifluoromethyl)phenyl)allyl carbonate ${ }^{3}(0.19 \mathrm{~g}, 0.63 \mathrm{mmol}, 2.1$ equiv),

$[\operatorname{Ir}(\mathrm{COD}) \mathrm{Cl}]_{2}(6.0 \mathrm{mg}, 0.0090 \mathrm{mmol}, 3.0 \mathrm{~mol} \%)$ and $\mathbf{L 3}(18 \mathrm{mg}, 0.036 \mathrm{mmol}, 12$ mol\%), AgOTf (3.9 mg, $0.015 \mathrm{mmol}, 5.0 \mathrm{~mol} \%$ ), bisborylmethane (80 mg, 0.30 mmol, 1.0 equiv), LiOtBu ( $36 \mathrm{mg}, 0.45 \mathrm{mmol}, 1.5$ equiv). The product was isolated as a yellow oil (77 mg, $0.24 \mathrm{mmol}, 79 \%)$. IR (thin film, $\mathbf{~ c m}^{-1}$ ) 2925, 1618, 1409, 1162, 800; ${ }^{1} \mathbf{H}$ NMR (400 MHz, $\left.\mathbf{C D C l}_{3}\right) \delta: 7.53(\mathrm{~d}, J=8.1 \mathrm{~Hz}, 2 \mathrm{H}), 7.34(\mathrm{~d}, J=8.1 \mathrm{~Hz}, 2 \mathrm{H}), 5.98(\mathrm{ddd}, J=17.1,10.2,6.8 \mathrm{~Hz}, 1 \mathrm{H}), 5.08-4.98(\mathrm{~m}$, 2H), 3.67 (ddd, $J=7.6,7.6,7.6 \mathrm{~Hz}, 1 \mathrm{H}), 1.32(\mathrm{dd}, J=15.4,8.1 \mathrm{~Hz}, 1 \mathrm{H}), 1.24(\mathrm{dd}, J=15.5,7.9 \mathrm{~Hz}, 1 \mathrm{H}), 1.14(\mathrm{~s}$,

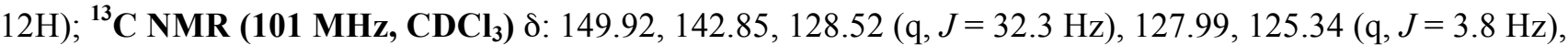
124.49 (q, $J=271.7 \mathrm{~Hz}), 113.84,83.41,44.95,24.87$, and 24.82; ${ }^{\mathbf{1 9}} \mathbf{F}$ NMR (376 MHz, CDCl 3 ) $\delta:-62.33$; HRMS (DART-TOF) calculated for $\mathrm{C}_{17} \mathrm{H}_{22} \mathrm{BF}_{4} \mathrm{O}_{2}{ }^{-}[\mathrm{M}+\mathrm{F}]^{-} \mathrm{m} / \mathrm{z} 345.1654$, found 345.1657 ; HPLC analysis of the product after being oxidized to the corresponding alcohol by $\mathrm{NaBO}_{3} \cdot 4 \mathrm{H}_{2} \mathrm{O}(\mathrm{AD}-\mathrm{H}$, Hexanes: $i \mathrm{PrOH}=99: 1,1$ $\mathrm{mL} / \mathrm{min}$ ) indicated $93 \%$ ee: $\mathrm{t}_{\mathrm{R}}($ major $)=20.6 \mathrm{~min}, \mathrm{t}_{\mathrm{R}}($ minor $)=25.7 \mathrm{~min} ;[\boldsymbol{\alpha}]_{\mathbf{D}}{ }^{13}=20.0\left(\mathrm{c}=0.075, \mathrm{CHCl}_{3}\right)$.<smiles>C=C[C@@H](CB1OC(C)(C)C(C)(C)O1)c1ccc(Cl)c(Cl)c1</smiles>

(R)-2-(2-(3,4-dichlorophenyl)but-3-enyl)-4,4,5,5-tetramethyl-1,3,2-dioxaborolane (12j). Following the General Procedure C using tert-butyl 1-(3,4-dichlorophenyl)allyl carbonate (0.19 g, 0.63 mmol, 2.1 equiv), $[\mathrm{Ir}(\mathrm{COD}) \mathrm{Cl}]_{2}(6.0 \mathrm{mg}, 0.0090 \mathrm{mmol}, 3.0 \mathrm{~mol} \%) \mathrm{L3}$ (18 mg, $\left.0.036 \mathrm{mmol}, 12 \mathrm{~mol} \%\right)$, AgOTf (3.9 mg, $0.015 \mathrm{mmol}$, $5.0 \mathrm{~mol} \%$ ), bisborylmethane ( $80 \mathrm{mg}, 0.30 \mathrm{mmol}, 1.0$ equiv), and LiOtBu ( $36 \mathrm{mg}, 0.45 \mathrm{mmol}, 1.5$ equiv). The product was isolated as a yellow oil (79 $\mathrm{mg}, 0.24 \mathrm{mmol}, 81 \%$ ). IR (thin film, $\mathbf{c m}^{-1}$ ) 3078, 1633, 1468, 1144, 847; ${ }^{1}$ H NMR (400 MHz, CDCl $)$ ) $:$ 7.34-7.32 (m, 2H), $7.06(\mathrm{dd}, J=8.3,2.0 \mathrm{~Hz}, 1 \mathrm{H}), 5.94(\mathrm{ddd}, J=17.0,10.2,6.8$ $\mathrm{Hz}, 1 \mathrm{H}), 5.08-4.99$ (m, 2H), 3.57 (ddd, $J=7.7,7.7,7.7 \mathrm{~Hz}, 1 \mathrm{H}), 1.29$ (dd, $J=18.2,10.4 \mathrm{~Hz}, 1 \mathrm{H}), 1.24-1.18$ (overlapping m, 1H), 1.16 (s, 12H); ${ }^{13} \mathbf{C}$ NMR (101 MHz, $\mathbf{C D C l}_{3}$ ) $\delta: ~ 146.14, ~ 142.56,132.19,130.29,129.95$, 129.81, 127.13, 113.98, 83.45, 44.23, and 24.88; HRMS (DART-TOF) calculated for $\mathrm{C}_{16} \mathrm{H}_{21} \mathrm{BCl}_{2} \mathrm{FO}_{2}{ }^{-}[\mathrm{M}+\mathrm{F}]^{-}$ $\mathrm{m} / \mathrm{z} 345.1001$, found 345.0993; HPLC analysis of the product after being oxidized to the corresponding alcohol by $\mathrm{NaBO}_{3} \cdot 4 \mathrm{H}_{2} \mathrm{O}(\mathrm{AD}-\mathrm{H}$, Hexanes: $i \mathrm{PrOH}=99: 1,1 \mathrm{~mL} / \mathrm{min})$ indicated $90 \%$ ee: $\mathrm{t}_{\mathrm{R}}$ (major) $=31.0$ min, $\mathrm{t}_{\mathrm{R}}$ (minor) $=34.7 \mathrm{~min} ;[\alpha]_{\mathbf{D}}{ }^{13}=15.5\left(\mathrm{c}=0.1, \mathrm{CHCl}_{3}\right)$. 


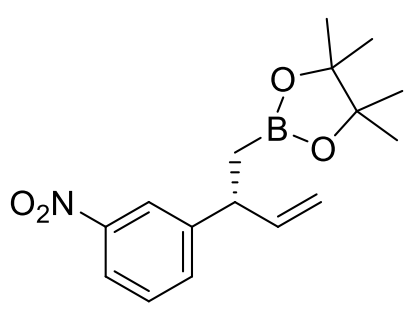

(R)-4,4,5,5-tetramethyl-2-(2-(3-nitrophenyl)but-3-enyl)-1,3,2-dioxaborolane (12k). Following the General Procedure C using tert-butyl 1-(3-nitrophenyl)allyl carbonate ${ }^{7}(0.18 \mathrm{~g}, 0.63 \mathrm{mmol}, 2.1 \text { equiv), [ } \operatorname{Ir}(\mathrm{COD}) \mathrm{Cl}]_{2}(6.0$ mg, $0.0090 \mathrm{mmol}, 3.0 \mathrm{~mol} \%), \mathbf{L 3}$ (18 mg, $0.036 \mathrm{mmol}, 12 \mathrm{~mol} \%)$, AgOTf (3.9 mg, $0.015 \mathrm{mmol}, 5.0 \mathrm{~mol} \%$ ), bisborylmethane ( $80 \mathrm{mg}, 0.30 \mathrm{mmol}, 1.0$ equiv), and $\mathrm{LiOtBu}(36 \mathrm{mg}, 0.45 \mathrm{mmol}, 1.5$ equiv). The product was isolated as a yellow oil (75 mg, 0.25 mmol, 82\%). IR (thin film, $\mathbf{c m}^{-1}$ ) 3080, 1638, 1530, 1143, 801; ${ }^{1} \mathbf{H}$ NMR $\left(400 \mathrm{MHz}, \mathbf{C D C l}_{3}\right) \delta: 8.12(\mathrm{t}, J=1.8 \mathrm{~Hz}, 1 \mathrm{H}), 8.04(\mathrm{ddd}, J=8.1,2.2,0.9 \mathrm{~Hz}, 1 \mathrm{H}), 7.57(\mathrm{~d}, J=7.7 \mathrm{~Hz}, 1 \mathrm{H}), 7.44$ $(\mathrm{t}, J=7.9 \mathrm{~Hz}, 1 \mathrm{H}), 6.00(\mathrm{ddd}, J=17.0,10.3,6.8 \mathrm{~Hz}, 1 \mathrm{H}), 5.13-5.03(\mathrm{~m}, 2 \mathrm{H}), 3.73(\mathrm{ddd}, J=7.6,7.6,7.6 \mathrm{~Hz}, 1 \mathrm{H})$, $1.35(\mathrm{dd}, J=15.5,7.8 \mathrm{~Hz}, 1 \mathrm{H}), 1.25(\mathrm{dd}, J=15.5,7.9 \mathrm{~Hz}, 1 \mathrm{H}), 1.150(\mathrm{~s}, 6 \mathrm{H}), 1.147(\mathrm{~s}, 6 \mathrm{H}) ;{ }^{13} \mathbf{C} \mathbf{N M R}(\mathbf{1 0 1}$ $\mathbf{M H z}, \mathbf{C D C l}_{3}$ ) $\delta: 148.26,147.84,142.12,133.89,129.11,122.66,121.23,114.31,83.36,44.55,24.723$, and 24.719; HRMS (DART-TOF) calculated for $\mathrm{C}_{16} \mathrm{H}_{22} \mathrm{BNFO}_{4}{ }^{-}[\mathrm{M}+\mathrm{F}]^{-} \mathrm{m} / \mathrm{z}$ 322.1631, found 322.1645; HPLC analysis of the product after being oxidized to the corresponding alcohol by $\mathrm{NaBO}_{3} \cdot 4 \mathrm{H}_{2} \mathrm{O}(\mathrm{AD}-\mathrm{H}$, Hexanes: $i \operatorname{PrOH}=99: 1,1 \mathrm{~mL} / \mathrm{min})$ indicated $91 \%$ ee: $\mathrm{t}_{\mathrm{R}}($ major $)=96.3 \mathrm{~min}, \mathrm{t}_{\mathrm{R}}($ minor $)=91.3 \mathrm{~min} ;[\alpha]_{\mathbf{D}}{ }^{13}=38.6(\mathrm{c}=0.1$, $\left.\mathrm{CHCl}_{3}\right)$.<smiles>C=C[C@@H](CB1OC(C)(C)C(C)(C)O1)c1cccc(C#N)c1</smiles>

(R)-3-(1-(4,4,5,5-tetramethyl-1,3,2-dioxaborolan-2-yl)but-3-en-2-yl)benzonitrile (12l). Following the General Procedure $\mathbf{C}$ using tert-butyl 1-(3-cyanophenyl)allyl carbonate $\left(0.16 \mathrm{~g}, 0.63 \mathrm{mmol}, 2.1\right.$ equiv), $[\operatorname{Ir}(\mathrm{COD}) \mathrm{Cl}]_{2}(6.0$ mg, $0.0090 \mathrm{mmol}, 3.0 \mathrm{~mol} \%$ ), $\mathbf{L 3}$ (18 mg, $0.036 \mathrm{mmol}, 12 \mathrm{~mol} \%$ ), AgOTf (3.9 mg, $0.015 \mathrm{mmol}, 5.0 \mathrm{~mol} \%$ ), bisborylmethane ( $80 \mathrm{mg}, 0.30 \mathrm{mmol}, 1.0$ equiv), and $\mathrm{LiOtBu}(36 \mathrm{mg}, 0.45 \mathrm{mmol}, 1.5$ equiv). The product was isolated as a yellow oil (72 mg, $0.26 \mathrm{mmol}, 85 \%$ ). IR (thin film, $\mathbf{c m}^{-1}$ ) 3081, 2230, 1637, 1600, 1144, 847; ${ }^{1} \mathbf{H}$ NMR (400 MHz, CDCl $)_{3}$ ): 7.53 (s, 1H), 7.49-7.45 (m, 2H), 7.37 (dd, $\left.J=8.3,7.1 \mathrm{~Hz}, 1 \mathrm{H}\right), 5.96$ (ddd, $J=17.6$, 9.8, $6.7 \mathrm{~Hz}, 1 \mathrm{H}), 5.10-5.00(\mathrm{~m}, 2 \mathrm{H}), 3.64(\mathrm{ddd}, J=7.7,7.7,7.7 \mathrm{~Hz}, 1 \mathrm{H}), 1.31$ (dd, $J=15.5,7.8 \mathrm{~Hz}, 1 \mathrm{H}), 1.21(\mathrm{dd}$,

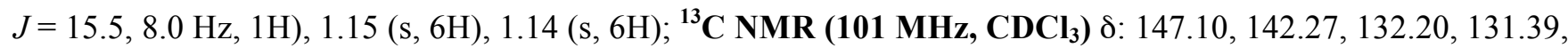
129.85, 129.04, 119.07, 114.09, 112.19, 83.33, 44.46, and 24.71; HRMS (DART-TOF) calculated for $\mathrm{C}_{17} \mathrm{H}_{22} \mathrm{BNO}_{2}{ }^{-}[\mathrm{M}+\mathrm{F}]^{-} \mathrm{m} / \mathrm{z} 302.1733$, found 302.1738 ; HPLC analysis of the product after being oxidized to the 
corresponding alcohol by $\mathrm{NaBO}_{3} \cdot 4 \mathrm{H}_{2} \mathrm{O}(\mathrm{AD}-\mathrm{H}$, Hexanes: $i \mathrm{PrOH}=99: 1,1 \mathrm{~mL} / \mathrm{min})$ indicated $92 \%$ ee: $\mathrm{t}_{\mathrm{R}}$ (major) $=117.2 \min , \mathrm{t}_{\mathrm{R}}($ minor $)=112.0 \mathrm{~min} ;[\boldsymbol{\alpha}]_{\mathbf{D}}{ }^{\mathbf{1 3}}=20.7\left(\mathrm{c}=0.1, \mathrm{CHCl}_{3}\right)$.

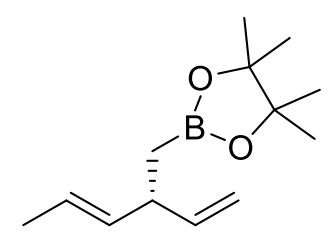

$(R, E)-4,4,5,5$-tetramethyl-2-(2-vinylpent-3-enyl)-1,3,2-dioxaborolane (12m). Following the General Procedure A using tert-butyl (2E,4E)-hexa-2,4-dienyl carbonate ${ }^{3}$ (60 mg, $0.30 \mathrm{mmol}, 1.0$ equiv), $\mathrm{Ag}_{3} \mathrm{PO}_{4}(3.5$ $\mathrm{mg}, 0.0083 \mathrm{mmol}, 2.8 \mathrm{~mol} \%$ ), bisborylmethane (10, $0.12 \mathrm{~g}, 0.45 \mathrm{mmol}, 1.5$ equiv), $\mathrm{LiO}^{t} \mathrm{Bu}$ (36 mg, $0.45 \mathrm{mmol}$, 1.5 equiv) and the stock solution of iridium catalyst $(0.6 \mathrm{~mL}, 0.015 \mathrm{mmol})$. The reaction was stirred at $50{ }^{\circ} \mathrm{C}$ for $36 \mathrm{~h}$. The product was isolated as a yellow oil (54 mg, $0.24 \mathrm{mmol}, 80 \%$ ). IR (thin film, $\mathbf{c m}^{-1}$ ) 3079, 2978, 1636,

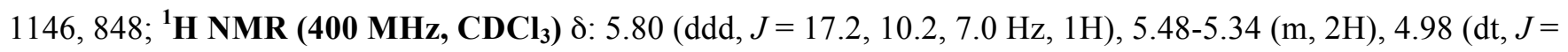
$17.2,1.6 \mathrm{~Hz}, 1 \mathrm{H}), 4.90(\mathrm{dt}, J=10.4,1.2 \mathrm{~Hz}, 1 \mathrm{H}), 2.96(\mathrm{dddd}, J=7.1,7.1,7.1,7.1 \mathrm{~Hz}, 1 \mathrm{H}), 1.64(\mathrm{~d}, J=5.4 \mathrm{~Hz}$, $3 \mathrm{H}), 1.23(\mathrm{~s}, 12 \mathrm{H}), 0.96(\mathrm{dd}, J=12.3,4.4 \mathrm{~Hz}, 1 \mathrm{H}), 0.91(\mathrm{dd}, J=12.3,4.9 \mathrm{~Hz}, 1 \mathrm{H}) ;{ }^{13} \mathbf{C} \mathbf{N M R}\left(\mathbf{1 0 1} \mathbf{M H z}, \mathbf{C D C l}_{3}\right)$

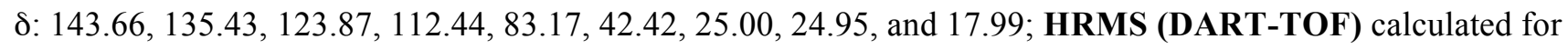
$\mathrm{C}_{13} \mathrm{H}_{23} \mathrm{BFO}_{2}{ }^{-}[\mathrm{M}+\mathrm{F}]^{-} \mathrm{m} / \mathrm{z} 241.1781$, found 241.1788; HPLC analysis of the product after being treated with a hydroboration/oxidation/dibenzoylation sequence (OD-H, Hexanes: $i \mathrm{PrOH}=99: 1,1 \mathrm{~mL} / \mathrm{min}$ ) indicated 93\% ee: $\mathrm{t}_{\mathrm{R}}($ major $)=16.5 \mathrm{~min}, \mathrm{t}_{\mathrm{R}}(\operatorname{minor})=18.9 \mathrm{~min} ; \quad[\alpha]_{\mathbf{D}}{ }^{13}=47.6\left(\mathrm{c}=0.1, \mathrm{CHCl}_{3}\right)$.

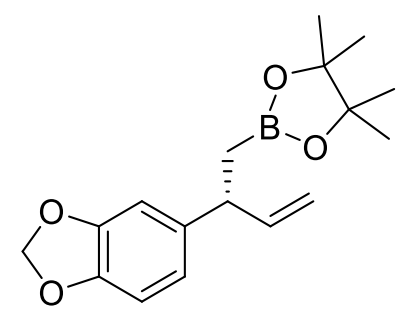

(R)-2-(2-(benzo[d][1,3]dioxol-5-yl)but-3-enyl)-4,4,5,5-tetramethyl-1,3,2-dioxaborolane (12n). Following the General Procedure A using (E)-3-(benzo[d][1,3]dioxol-5-yl)allyl tert-butyl carbonate ${ }^{5}(84 \mathrm{mg}, 0.30 \mathrm{mmol}, 1.0$ equiv), $\mathrm{Ag}_{3} \mathrm{PO}_{4}$ (2.1 mg, $0.0050 \mathrm{mmol}, 1.7 \mathrm{~mol} \%$ ), bisborylmethane (10, $\left.0.12 \mathrm{~g}, 0.45 \mathrm{mmol}, 1.5 \mathrm{equiv}\right), \mathrm{LiO}^{t} \mathrm{Bu}$ (36 mg, $0.45 \mathrm{mmol}, 1.5$ equiv) and stock solution $(0.6 \mathrm{~mL}, 0.009 \mathrm{mmol})$. The product was isolated as a yellow oil (58 mg, 0.19 mmol, 64\%). IR (thin film, $\mathbf{c m}^{-1}$ ) 3076, 1637, 1504, 1145, 848; ${ }^{1} \mathbf{H}_{\text {NMR (400 MHz, } \mathbf{C D C l}}$ ) $\delta$ : 6.75-6.66 (m, 3H), $5.95(\mathrm{ddd}, J=11.0,8.9,3.7 \mathrm{~Hz}, 1 \mathrm{H}), 5.90(\mathrm{~s}, 2 \mathrm{H}), 5.02(\mathrm{dt}, J=17.1,1.5 \mathrm{~Hz}, 1 \mathrm{H}), 4.96(\mathrm{dt}, J=$ 10.4, $1.2 \mathrm{~Hz}, 1 \mathrm{H}), 3.53(\mathrm{ddd}, J=7.8,7.8,7.8 \mathrm{~Hz}, 1 \mathrm{H}), 1.25(\mathrm{dd}, J=15.3,8.2 \mathrm{~Hz}, 1 \mathrm{H}), 1.21-1.15(\mathrm{~m}, 1 \mathrm{H}), 1.16$ (s,

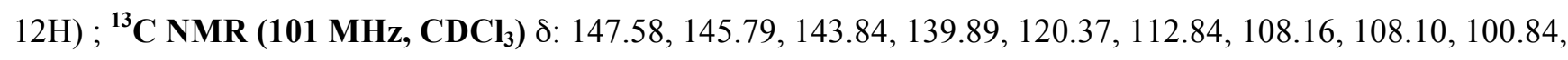
83.25, 44.78, 24.90, and 24.86; HRMS (DART-TOF) calculated for $\mathrm{C}_{17} \mathrm{H}_{23} \mathrm{BFO}_{4}{ }^{-}[\mathrm{M}+\mathrm{F}]^{-} \mathrm{m} / \mathrm{z} 321.1679$, found 321.1692; HPLC analysis of the product after being oxidized to the corresponding alcohol by $\mathrm{NaBO}_{3} \cdot 4 \mathrm{H}_{2} \mathrm{O}(\mathrm{AS}-$ 
H, Hexanes: $i \mathrm{PrOH}=90: 10,1 \mathrm{~mL} / \mathrm{min})$ indicated $92 \%$ ee: $\mathrm{t}_{\mathrm{R}}($ major $)=16.6 \mathrm{~min}, \mathrm{t}_{\mathrm{R}}(\operatorname{minor})=13.3 \mathrm{~min} ;[\boldsymbol{\alpha}]_{\mathbf{D}}{ }^{13}=$ $10.5\left(\mathrm{c}=0.1, \mathrm{CHCl}_{3}\right)$.

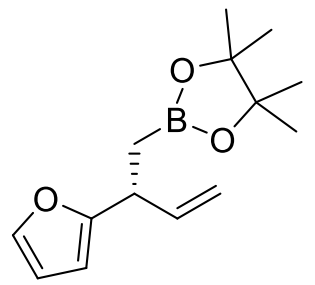

(R)-2-(2-(furan-2-yl)but-3-enyl)-4,4,5,5-tetramethyl-1,3,2-dioxaborolane (120). Following the General Procedure B using (E)-tert-butyl 3-(furan-2-yl)allyl carbonate ${ }^{3}$ (67 mg, 0.30 mmol, 1.0 equiv), $\mathrm{Ag}_{3} \mathrm{PO}_{4}(2.1 \mathrm{mg}$, $0.0050 \mathrm{mmol}, 1.7 \mathrm{~mol} \%$ ), bisborylmethane (10, $0.12 \mathrm{~g}, 0.45 \mathrm{mmol}, 1.5$ equiv), $\mathrm{LiO}^{t} \mathrm{Bu}$ (36 mg, $0.45 \mathrm{mmol}, 1.5$ equiv), $[\operatorname{Ir}(\mathrm{COD}) \mathrm{Cl}]_{2}(6.0 \mathrm{mg}, 0.0090 \mathrm{mmol}, 3.0 \mathrm{~mol} \%)$ and $(11 \mathrm{bS})-N$-benzhydryl- $N$-phenyldinaphtho[2,1-d:1',2'$f][1,3,2]$ dioxaphosphepin-4-amine ${ }^{1}$ as ligand $(10 \mathrm{mg}, 0.018 \mathrm{mmol}, 6.0 \mathrm{~mol} \%)$. The product was isolated as a yellow oil (63 mg, $0.25 \mathrm{mmol}, 84 \%$ ). IR (thin film, $\mathbf{~ c m}^{-1}$ ) 2980, 1505, 1366, 1144, 848; ${ }^{1} \mathrm{H} \mathrm{NMR} \mathrm{(400} \mathrm{MHz,}$ $\left.\mathbf{C D C l}_{3}\right) \delta: 7.30(\mathrm{dd}, J=1.8,0.7 \mathrm{~Hz}, 1 \mathrm{H}), 6.26(\mathrm{dd}, J=3.1,1.9 \mathrm{~Hz}, 1 \mathrm{H}), 6.01(\mathrm{~d}, J=3.2 \mathrm{~Hz}, 1 \mathrm{H}), 5.93(\mathrm{ddd}, J=$ 17.3, 10.1, $7.4 \mathrm{~Hz}, 1 \mathrm{H}), 5.08(\mathrm{dt}, J=17.1,1.3 \mathrm{~Hz}, 1 \mathrm{H}), 5.04-5.00(\mathrm{~m}, 1 \mathrm{H}), 3.67(\mathrm{ddd}, J=7.7,7.7,7.7 \mathrm{~Hz}, 1 \mathrm{H})$, $1.30(\mathrm{dd}, J=15.4,7.6 \mathrm{~Hz}, 1 \mathrm{H}), 1.25-1.22(\mathrm{~m}, 1 \mathrm{H}), 1.21(\mathrm{~s}, 6 \mathrm{H}), 1.20(\mathrm{~s}, 6 \mathrm{H}) ;{ }^{13} \mathbf{C}$ NMR (101 MHz, CDCl$)_{3} \delta:$ 158.71, 141.10, 140.87, 114.29, 110.06, 104.35, 83.33, 38.98, 24.92, and 24.87; HRMS (DART-TOF) calculated for $\mathrm{C}_{14} \mathrm{H}_{21} \mathrm{BO}_{3}[\mathrm{M}+\mathrm{H}]^{+} \mathrm{m} / \mathrm{z} 249.1662$, found 249.1669. HPLC analysis of the product after being oxidized to the corresponding alcohol by $\mathrm{NaBO}_{3} \cdot 4 \mathrm{H}_{2} \mathrm{O}(\mathrm{AS}-\mathrm{H}$, Hexanes: $i \mathrm{PrOH}=99: 1,1 \mathrm{~mL} / \mathrm{min})$ indicated $92 \%$ ee: $\mathrm{t}_{\mathrm{R}}$ (major) $=25.1 \mathrm{~min}, \mathrm{t}_{\mathrm{R}}($ minor $)=24.0 \mathrm{~min} ;[\boldsymbol{\alpha}]_{\mathbf{D}}{ }^{13}=-34.5\left(\mathrm{c}=0.1, \mathrm{CHCl}_{3}\right)$.

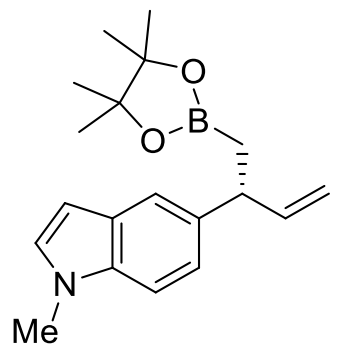

(R)-1-methyl-5-(1-(4,4,5,5-tetramethyl-1,3,2-dioxaborolan-2-yl)but-3-en-2-yl)-1H-indole (12p). Following the General Procedure A using (E)-tert-butyl 3-(1-methyl-1H-indol-5-yl)allyl carbonate (86 mg, $0.30 \mathrm{mmol}, 1.0$ equiv), $\mathrm{Ag}_{3} \mathrm{PO}_{4}$ (2.1 mg, $0.0050 \mathrm{mmol}, 1.7 \mathrm{~mol} \%$ ), bisborylmethane (10, $0.12 \mathrm{~g}, 0.45 \mathrm{mmol}, 1.5$ equiv), $\mathrm{LiO}^{t} \mathrm{Bu}$ (36 $\mathrm{mg}, 0.45 \mathrm{mmol}, 1.5$ equiv) and stock solution $(0.6 \mathrm{~mL}, 0.009 \mathrm{mmol})$. The product was isolated as a yellow oil (78 mg, 0.25 mmol, 84\%). IR (thin film, $\mathbf{c m}^{-1}$ ) 2977, 1636, 1514, 1143, 848; ${ }^{1} \mathbf{H ~ N M R ~ ( 4 0 0 ~ M H z , ~ C D C l}_{3}$ ) $\delta$ : $7.46(\mathrm{~d}, J=1.2 \mathrm{~Hz}, 1 \mathrm{H}), 7.22(\mathrm{~d}, J=8.5 \mathrm{~Hz}, 1 \mathrm{H}), 7.10(\mathrm{dd}, J=8.5,1.6 \mathrm{~Hz}, 1 \mathrm{H}), 6.99$ (d, $J=3.1 \mathrm{~Hz}, 1 \mathrm{H}), 6.40$ (d, $J=2.6 \mathrm{~Hz}, 1 \mathrm{H}), 6.07(\mathrm{ddd}, J=17.1,10.2,6.8 \mathrm{~Hz}, 1 \mathrm{H}), 5.05(\mathrm{dt}, J=17.1,1.5 \mathrm{~Hz}, 1 \mathrm{H}), 4.95(\mathrm{dt}, J=10.4,1.2 \mathrm{~Hz}$, 
$1 \mathrm{H}), 3.75$ (s, 3H), $3.72(\mathrm{ddd}, J=7.4,7.4,7.4 \mathrm{~Hz}, 1 \mathrm{H}) 1.36(\mathrm{dd}, J=15.2,8.6 \mathrm{~Hz}, 1 \mathrm{H}), 1.30$ (dd, $J=15.8,8.0 \mathrm{~Hz}$, 1H), 1.15 (s, 6H), 1.14 (s, 6H); ${ }^{13} \mathbf{C}$ NMR (101 MHz, $\mathbf{C D C l}_{3}$ ) $\delta: ~ 144.78,136.85,135.65,128.88,128.66,121.75$, 119.18, 112.24, 109.05, 100.83, 83.15, 45.09, 32.95, 24.93, and 24.88; HRMS (DART-TOF) calculated for $\mathrm{C}_{19} \mathrm{H}_{26} \mathrm{BNO}_{2}[\mathrm{M}+\mathrm{H}]^{+} \mathrm{m} / \mathrm{z}$ 312.2135, found 312.2149. HPLC analysis (AD-H, Hexanes: $i \mathrm{PrOH}=99: 1,1$ $\mathrm{mL} / \mathrm{min})$ indicated $95 \%$ ee: $\mathrm{t}_{\mathrm{R}}($ major $)=5.4 \mathrm{~min}, \mathrm{t}_{\mathrm{R}}($ minor $)=4.8 \mathrm{~min} ;[\boldsymbol{\alpha}]_{\mathbf{D}}{ }^{13}=29.155\left(\mathrm{c}=0.2, \mathrm{CHCl}_{3}\right)$.<smiles>C=C[C@@H](CB1OC(C)(C)C(C)(C)O1)c1ccc2ccn(C)c2c1</smiles>

(R)-1-methyl-6-(1-(4,4,5,5-tetramethyl-1,3,2-dioxaborolan-2-yl)but-3-en-2-yl)-1H-indole (12q). Following the General Procedure A using (E)-tert-butyl 3-(1-methyl-1H-indol-6-yl)allyl carbonate (86 mg, $0.30 \mathrm{mmol}, 1.0$ equiv), $\mathrm{Ag}_{3} \mathrm{PO}_{4}$ (2.1 mg, $0.0050 \mathrm{mmol}, 1.7 \mathrm{~mol} \%$ ), bisborylmethane (10, $\left.0.12 \mathrm{~g}, 0.45 \mathrm{mmol}, 1.5 \mathrm{equiv}\right), \mathrm{LiO}^{t} \mathrm{Bu}$ (36 mg, $0.45 \mathrm{mmol}, 1.5$ equiv) and stock solution $(0.6 \mathrm{~mL}, 0.009 \mathrm{mmol})$. The product was isolated as a light yellow solid (86 mg, $0.28 \mathrm{mmol}, 92 \%$ ). Melting point: 67-68 ${ }^{\circ} \mathrm{C}$; IR (thin film, $\mathbf{c m}^{-1}$ ) 2977, 1637, 1606, 1143, 846; ${ }^{1} \mathbf{H}$ NMR (400 MHz, $\mathbf{C D C l}_{3}$ ) $\delta: 7.51$ (d, $\left.J=8.2 \mathrm{~Hz}, 1 \mathrm{H}\right), 7.17$ (s, 1H), $7.00(\mathrm{dd}, J=8.2,1.4 \mathrm{~Hz}, 1 \mathrm{H}), 6.97$ (d, $J=3.1 \mathrm{~Hz}, 1 \mathrm{H}), 6.41(\mathrm{dd}, J=3.1,0.6 \mathrm{~Hz}, 1 \mathrm{H}), 6.09$ (ddd, $J=17.1,10.2,6.8 \mathrm{~Hz}, 1 \mathrm{H}), 5.08$ (dt, $J=17.1,1.5 \mathrm{~Hz}$, 1H), 4.98 (dt, $J=10.4,1.6 \mathrm{~Hz}, 1 \mathrm{H}), 3.753$ (ddd, $J=7.5,7.5,7.5 \mathrm{~Hz}, 1 \mathrm{H}), 3.746$ (s, 3H), 1.43-1.27 (m, 2H), 1.15 (s, 6H), 1.14 (s, 6H); ${ }^{13} \mathbf{C}$ NMR (101 MHz, $\mathbf{C D C l}_{3}$ ) $\delta:$ 144.42, 139.63, 137.01, 128.56, 126.92, 120.66, 119.62, 112.53, 107.65, 100.66, 83.17, 45.44, 32.86, 24.93, and 24.85; HRMS (DART-TOF) calculated for $\mathrm{C}_{19} \mathrm{H}_{26} \mathrm{BNO}_{2}$ $[\mathrm{M}+\mathrm{H}]^{+} \mathrm{m} / \mathrm{z}$ 312.2135, found 312.2142. HPLC analysis (AD-H, Hexanes: $i \mathrm{PrOH}=99: 1,1 \mathrm{~mL} / \mathrm{min}$ ) indicated $93 \%$ ee: $\mathrm{t}_{\mathrm{R}}($ major $)=4.5 \mathrm{~min}, \mathrm{t}_{\mathrm{R}}($ minor $)=4.0 \mathrm{~min} ;[\alpha]_{\mathbf{D}}{ }^{13}=32.0\left(\mathrm{c}=0.2, \mathrm{CHCl}_{3}\right)$.

\section{ORTEP rendering for $12 q$}

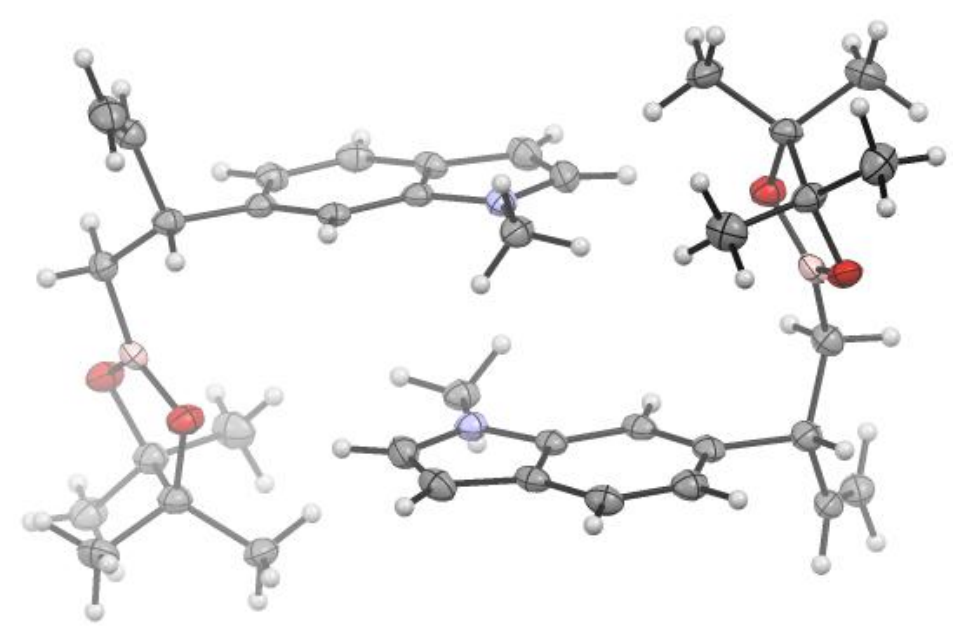




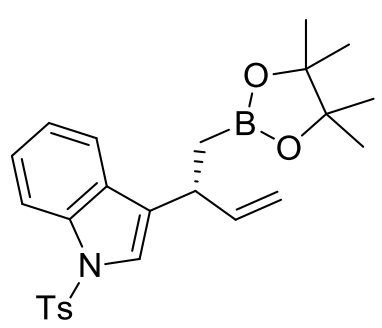

(R)-3-(1-(4,4,5,5-tetramethyl-1,3,2-dioxaborolan-2-yl)but-3-en-2-yl)-1-tosyl-1H-indole (12r). Following the General Procedure A using (E)-tert-butyl 3-(1-tosyl-1H-indol-3-yl)allyl carbonate $(0.13 \mathrm{~g}, 0.30 \mathrm{mmol}, 1.0$ equiv), $\mathrm{Ag}_{3} \mathrm{PO}_{4}$ (2.1 mg, $0.0050 \mathrm{mmol}, 1.7 \mathrm{~mol} \%$ ), bisborylmethane (10, $\left.0.12 \mathrm{~g}, 0.45 \mathrm{mmol}, 1.5 \mathrm{equiv}\right), \mathrm{LiO}^{t} \mathrm{Bu}$ (36 mg, $0.45 \mathrm{mmol}, 1.5$ equiv) and stock solution $(0.6 \mathrm{~mL}, 0.009 \mathrm{mmol})$. The product was isolated as a yellow oil

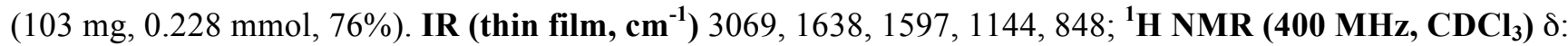
$7.95(\mathrm{~d}, J=8.3 \mathrm{~Hz}, 1 \mathrm{H}), 7.73(\mathrm{~d}, J=8.3 \mathrm{~Hz}, 2 \mathrm{H}), 7.54(\mathrm{~d}, J=7.8 \mathrm{~Hz}, 1 \mathrm{H}), 7.36(\mathrm{~s}, 1 \mathrm{H}), 7.30-7.24(\mathrm{~m}, 1 \mathrm{H}), 7.21-$ $7.15(\mathrm{~m}, 3 \mathrm{H}), 5.95$ (ddd, $J=17.2,10.1,7.1 \mathrm{~Hz}, 1 \mathrm{H}), 5.05(\mathrm{br} \mathrm{d}, J=17.1 \mathrm{~Hz}, 1 \mathrm{H}), 4.99$ (br d, $J=10.1 \mathrm{~Hz}, 1 \mathrm{H})$, $3.79(\mathrm{ddd}, J=7.5,7.5,7.5 \mathrm{~Hz}, 1 \mathrm{H}), 2.32(\mathrm{~s}, 3 \mathrm{H}), 1.36(\mathrm{dd}, J=14.0,5.8 \mathrm{~Hz}, 1 \mathrm{H}), 1.31(\mathrm{dd}, J=13.9,6.9 \mathrm{~Hz}, 1 \mathrm{H})$, 1.17 (s, 6H), and 1.15 (s, 6H); ${ }^{13} \mathbf{C}$ NMR (101 MHz, $\left.\mathbf{C D C l}_{3}\right)$ 8: 144.76, 142.02, 135.64, 135.60, 130.47, 129.88, $127.09,126.86,124.57,122.90,122.57,120.58,114.02,113.76,83.39,36.31,24.93,24.81$, and 21.65; HRMS (DART-TOF) calculated for $\mathrm{C}_{25} \mathrm{H}_{30} \mathrm{BNO}_{4} \mathrm{~S}\left[\mathrm{M}+\mathrm{NH}_{4}\right]^{+} \mathrm{m} / \mathrm{z}$ 469.2332, found 469.2359. HPLC analysis (AD-H, Hexanes: $i \mathrm{PrOH}=95: 5,1 \mathrm{~mL} / \mathrm{min})$ indicated $86 \%$ ee: $\mathrm{t}_{\mathrm{R}}($ major $)=10.9 \mathrm{~min}, \mathrm{t}_{\mathrm{R}}(\operatorname{minor})=8.4 \mathrm{~min} ;[\boldsymbol{\alpha}]_{\mathbf{D}}{ }^{13}=-17.2$ $\left(\mathrm{c}=0.1, \mathrm{CHCl}_{3}\right)$.

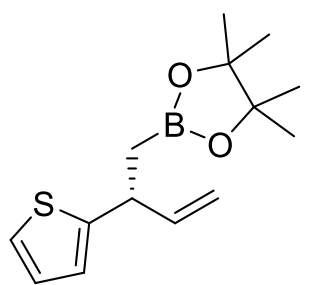

(R)-4,4,5,5-tetramethyl-2-(2-(thiophen-2-yl)but-3-enyl)-1,3,2-dioxaborolane (12s). Following the General Procedure A using (E)-tert-butyl 3-(thiophen-2-yl)allyl carbonate ${ }^{8}$ (72 mg, 0.30 mmol, 1.0 equiv), Ag $_{3} \mathrm{PO}_{4}(2.1$ $\mathrm{mg}, 0.0050 \mathrm{mmol}, 1.7 \mathrm{~mol} \%$ ), bisborylmethane (10, $0.12 \mathrm{~g}, 0.45 \mathrm{mmol}, 1.5$ equiv), $\mathrm{LiO}^{t} \mathrm{Bu}$ (36 mg, $0.45 \mathrm{mmol}$, 1.5 equiv) and stock solution $(0.6 \mathrm{~mL}, 0.009 \mathrm{mmol})$. The product was isolated as a yellow oil $(79 \mathrm{mg}, 0.30 \mathrm{mmol}$, 99\%). IR (thin film, $\mathbf{c m}^{-1}$ ) 3078, 1638, 1479, 1145, 847; ${ }^{1} \mathbf{H}$ NMR (400 MHz, $\mathbf{C D C l}_{3}$ ) $\delta: 7.11$ (dd, $J=5.1,1.2$ $\mathrm{Hz}, 1 \mathrm{H}), 6.90$ (dd, $J=5.1,3.5 \mathrm{~Hz}, 1 \mathrm{H}), 6.82$ (dt, $J=3.6,0.8 \mathrm{~Hz}, 1 \mathrm{H}), 5.99$ (ddd, $J=17.3,10.1,7.4 \mathrm{~Hz}, 1 \mathrm{H}), 5.09$ (dt, $J=17.0,1.3 \mathrm{~Hz}, 1 \mathrm{H}), 4.99(\mathrm{dt}, J=10.0,1.2 \mathrm{~Hz}, 1 \mathrm{H}), 3.88(\mathrm{ddd}, J=7.4,7.4,7.4 \mathrm{~Hz}, 1 \mathrm{H}), 1.37$ (dd, $J=14.8$,

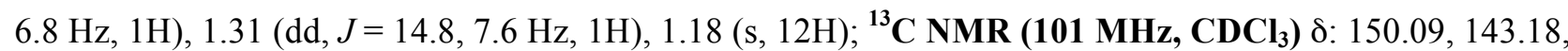
126.60, 123.23, 123.22, 113.51, 83.35, 40.63, 24.94, and 24.82; HRMS (DART-TOF) calculated for $\mathrm{C}_{14} \mathrm{H}_{21} \mathrm{BO}_{2} \mathrm{~S}$ $[\mathrm{M}+\mathrm{H}]^{+} \mathrm{m} / \mathrm{z}$ 265.1434, found 265.1421. HPLC analysis of the product after being oxidized to the corresponding 
alcohol by $\mathrm{NaBO}_{3} \cdot 4 \mathrm{H}_{2} \mathrm{O}($ AS-H, Hexanes: $i \mathrm{PrOH}=99: 1,1 \mathrm{~mL} / \mathrm{min})$ indicated $98 \%$ ee: $\mathrm{t}_{\mathrm{R}}$ (major) $=25.5 \mathrm{~min}, \mathrm{t}_{\mathrm{R}}$ $($ minor $)=23.7 \mathrm{~min} ;[\alpha]_{\mathbf{D}}{ }^{13}=-29.2\left(\mathrm{c}=0.1, \mathrm{CHCl}_{3}\right)$.

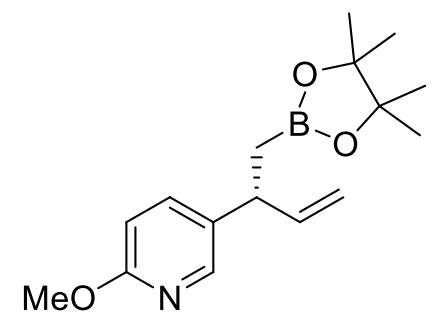

(R)-2-methoxy-5-(1-(4,4,5,5-tetramethyl-1,3,2-dioxaborolan-2-yl)but-3-en-2-yl)pyridine (12t). Following the General Procedure A using (E)-tert-butyl 3-(6-methoxypyridin-3-yl)allyl carbonate (80 mg, $0.30 \mathrm{mmol}, 1.0$ equiv), $\mathrm{Ag}_{3} \mathrm{PO}_{4}$ (2.1 mg, $\left.0.0050 \mathrm{mmol}, 1.7 \mathrm{~mol} \%\right)$, bisborylmethane (10, $\left.0.12 \mathrm{~g}, 0.45 \mathrm{mmol}, 1.5 \mathrm{equiv}\right), \mathrm{LiO}^{t} \mathrm{Bu}$ (36 mg, $0.45 \mathrm{mmol}, 1.5$ equiv) and stock solution $(0.6 \mathrm{~mL}, 0.009 \mathrm{mmol})$. The product was isolated as a yellow oil (78 mg, 0.27 mmol, 90\%). IR (thin film, $\mathbf{c m}^{-1}$ ) 2977, 1513, 1468, 1144, 848; ${ }^{1} \mathbf{H ~ N M R ~ ( 4 0 0 ~ M H z , ~ C D C l}_{3}$ ) $\delta$ : $8.02(\mathrm{~d}, J=2.4 \mathrm{~Hz}, 1 \mathrm{H}), 7.43(\mathrm{dd}, J=8.5,2.5 \mathrm{~Hz}, 1 \mathrm{H}), 6.67$ (d, $J=8.5 \mathrm{~Hz}, 1 \mathrm{H}), 5.96$ (ddd, $J=17.0,10.2,6.6 \mathrm{~Hz}$, $1 \mathrm{H}), 5.06-4.95(\mathrm{~m}, 2 \mathrm{H}), 3.91(\mathrm{~s}, 3 \mathrm{H}), 3.57$ (ddd, $J=7.8,7.8,7.8 \mathrm{~Hz}, 1 \mathrm{H}), 1.29(\mathrm{dd}, J=15.4,8.1 \mathrm{~Hz}, 1 \mathrm{H}), 1.21(\mathrm{dd}$, $J=15.6,7.6 \mathrm{~Hz}, 1 \mathrm{H}), 1.16$ (s, 12H); ${ }^{13} \mathbf{C} \mathbf{N M R}\left(\mathbf{1 0 1} \mathbf{M H z}, \mathbf{C D C l}_{3}\right.$ ) $\delta: 162.92,145.73,143.29,138.10,133.65$, 113.41, 110.53, 83.37, 53.45, 41.64, and 24.88; HRMS (DART-TOF) calculated for $\mathrm{C}_{16} \mathrm{H}_{24} \mathrm{BNO}_{3}[\mathrm{M}+\mathrm{H}]^{+} \mathrm{m} / \mathrm{z}$ 290.1927, found 290.1928. HPLC analysis of the product after being oxidized to the corresponding alcohol by $\mathrm{NaBO}_{3} \cdot 4 \mathrm{H}_{2} \mathrm{O}($ AS-H, Hexanes: $i \mathrm{PrOH}=90: 10,1 \mathrm{~mL} / \mathrm{min})$ indicated 94\% ee: $\mathrm{t}_{\mathrm{R}}($ major$)=16.2 \mathrm{~min}, \mathrm{t}_{\mathrm{R}}($ minor $)=$ $9.1 \mathrm{~min} ;[\alpha]_{\mathbf{D}}{ }^{13}=-16.2\left(\mathrm{c}=0.2, \mathrm{CHCl}_{3}\right)$. 


\section{Characterization Data for Products in Scheme 2.}

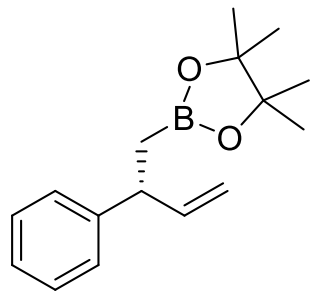

17

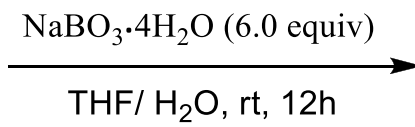

$\mathrm{THF} / \mathrm{H}_{2} \mathrm{O}, \mathrm{rt}, 12 \mathrm{~h}$

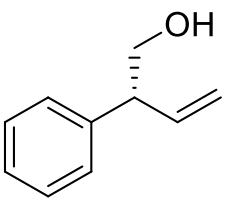

21

(R)-2-phenylbut-3-en-1-ol (21). ${ }^{9}$ A solution of $17(78 \mathrm{mg}, 0.30 \mathrm{mmol})$ in THF $(2.0 \mathrm{~mL})$ and $\mathrm{H}_{2} \mathrm{O}(2.0 \mathrm{~mL})$ was added $\mathrm{NaBO}_{3} \cdot 4 \mathrm{H}_{2} \mathrm{O}(0.28 \mathrm{~g}, 1.8 \mathrm{mmol}, 6.0$ equiv) at room temperature. The reaction mixture was stirred for $12 \mathrm{~h}$ and quenched by addition of saturated aq. $\mathrm{Na}_{2} \mathrm{~S}_{2} \mathrm{O}_{3}(5.0 \mathrm{~mL})$. The mixture was extracted with ethyl acetate $(3 \mathrm{X}$ $10 \mathrm{~mL})$. The combined organic layers were washed with water and brine, dried $\left(\mathrm{Na}_{2} \mathrm{SO}_{4}\right)$ and concentrated in vacuo to give a crude product. Purification by flash column chromatography (silica gel, eluting with petroleum ether/ethyl acetate, 20:1 to 10:1) afforded the known (R)-2-phenylbut-3-en-1-ol (21) as a light yellow solid (40

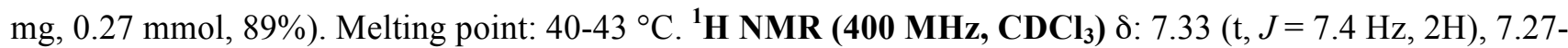
$7.19(\mathrm{~m}, 3 \mathrm{H}), 6.00(\mathrm{ddd}, J=17.7,10.4,7.7 \mathrm{~Hz}, 1 \mathrm{H}), 5.24-5.13(\mathrm{~m}, 2 \mathrm{H}), 3.80(\mathrm{~d}, J=7.0 \mathrm{~Hz}, 2 \mathrm{H}), 3.52(\mathrm{q}, J=7.3$ $\mathrm{Hz}, 1 \mathrm{H}) ;{ }^{13} \mathbf{C}$ NMR (101 MHz, $\mathbf{C D C l}_{3}$ ) $\delta: ~ 140.66,138.26,128.79,128.00,126.98,117.14,66.08$, and 52.54; HPLC analysis (AD-H, Hexanes: $i \mathrm{PrOH}=99.6: 0.4,1 \mathrm{~mL} / \mathrm{min})$ indicated 93\% ee: $\mathrm{t}_{\mathrm{R}}$ (major) $=45.4 \mathrm{~min}, \mathrm{t}_{\mathrm{R}}$ $(\operatorname{minor})=39.2 \mathrm{~min}$.

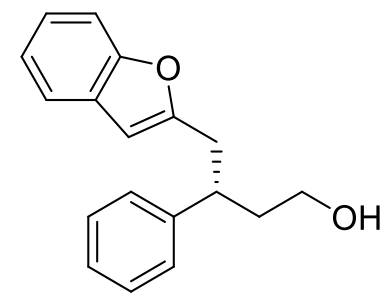

(S)-4-(benzofuran-2-yl)-3-phenylbutan-1-ol (23). To a solution of benzofuran (36 mg, $0.30 \mathrm{mmol}, 1.5$ equiv) in THF $(1 \mathrm{~mL})$ cooled at $-78{ }^{\circ} \mathrm{C}$ was added $\mathrm{nBuLi}(2.5 \mathrm{M}$ in hexanes, $0.12 \mathrm{~mL}, 0.30 \mathrm{mmol}, 1.5 \mathrm{equiv})$. The resulting solution was warmed to rt and stirred for $1 \mathrm{~h}$. The solution was cooled at $-78^{\circ} \mathrm{C}$ again, and a solution of 17 (52 $\mathrm{mg}, 0.2 \mathrm{mmol}, 1.0$ equiv) in THF $(0.5 \mathrm{~mL})$ was added slowly. The resulting solution was stirred at $-78{ }^{\circ} \mathrm{C}$ for $1 \mathrm{~h}$, at which time NBS (50 mg, $0.30 \mathrm{mmol}, 1.5$ equiv) in THF $(1.0 \mathrm{~mL})$ was added. After $1 \mathrm{~h}$ at $-78{ }^{\circ} \mathrm{C}$, saturated aqueous $\mathrm{Na}_{2} \mathrm{~S}_{2} \mathrm{O}_{3}$ was added. The resulting mixture was warmed to rt, and separated with between $\mathrm{H}_{2} \mathrm{O}$ and EtOAc. The aqueous layer was extracted once with EtOAc. The combined organic layers were dried, concentrated, and passed through a short pad of silica gel to give a colorless oil, which was used in the next step without further 
purification. To the product obtained was added THF $(0.5 \mathrm{~mL})$ and 9-BBN $(0.5 \mathrm{M}, 0.9 \mathrm{~mL})$. The resulting solution was left at $\mathrm{rt}$ for $4 \mathrm{~h} . \mathrm{H}_{2} \mathrm{O}_{2}$ and $\mathrm{NaOH}$ was added, and the resulting mixture was stirred at rt overnight. After extractive workup and column chromatography, compound $\mathbf{2 3}$ was isolated as a colorless oil (31 $\mathrm{mg}, 59 \%$

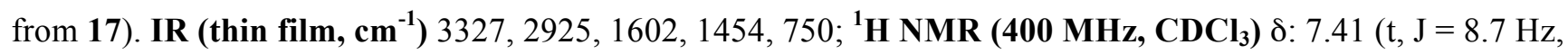
2H), 7.32-7.13 (m, 7H), $6.26(\mathrm{~s}, 1 \mathrm{H}), 3.59-3.52(\mathrm{~m}, 1 \mathrm{H}), 3.50-3.44(\mathrm{~m}, 1 \mathrm{H}), 3.34-3.24(\mathrm{~m}, 1 \mathrm{H}), 3.08(\mathrm{~d}, \mathrm{~J}=7.5 \mathrm{~Hz}$,

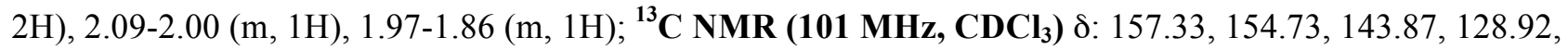
$128.75,127.60,126.79,123.35,122.54,120.45,110.89$, 103.61, 61.06, 41.28, 38.59, and 36.28; HPLC analysis (OD-H, Hexanes: $i \mathrm{PrOH}=70: 30,1 \mathrm{~mL} / \mathrm{min})$ indicated $92 \%$ ee: $\mathrm{t}_{\mathrm{R}}($ major$)=5.5 \mathrm{~min}, \mathrm{t}_{\mathrm{R}}($ minor $)=6.5 \mathrm{~min} ;[\alpha]_{\mathbf{D}}{ }^{13}$ $=94.3\left(\mathrm{c}=0.1, \mathrm{CHCl}_{3}\right)$.<smiles>C=C[C@H](CNC(=O)OCc1ccccc1)c1ccccc1</smiles>

(R)-tert-butyl 2-phenylbut-3-enylcarbamate (24). ${ }^{10}$ To a flame-dried test tube equipped with a magnetic stir bar and septum was added O-methylhydroxylamine solution ${ }^{11}(0.25 \mathrm{~mL}, 3.9 \mathrm{M}$ in THF as determined by ${ }^{1} \mathrm{H}$ NMR using 1,3,5-trimethoxybenzene as internal standard) and THF (2 mL) under $\mathrm{N}_{2}$ atomsphere. The flask was cooled to $-78^{\circ} \mathrm{C}$. $n \mathrm{BuLi}(0.4 \mathrm{~mL}, 1.0 \mathrm{mmol}, 2.5 \mathrm{M}$ in hexanes $)$ was added to the test tube dropwise and the solution was allowed to stir at $-78^{\circ} \mathrm{C}$ for $30 \mathrm{~min}$. Then, a solution of 17 (78 $\mathrm{mg}, 0.30$ $\mathrm{mmol})$ in THF $(1.0 \mathrm{~mL})$ was added dropwise to the test tube, which was then allowed to warm to rt, and further to $60{ }^{\circ} \mathrm{C}$. The test tube was kept at $60^{\circ} \mathrm{C}$ for $12 \mathrm{~h}$. The volatiles in the reaction was then removed under vacuum. To the residue was added $\mathrm{CHCl}_{3}(1 \mathrm{~mL})$ and $\mathrm{Boc}_{2} \mathrm{O}(0.3 \mathrm{~mL}, 1.3 \mathrm{mmol})$ at $\mathrm{rt}$. After stirring for $1 \mathrm{~h}$ the reaction mixture was concentrated and subjected to column chromatography (hexanes:EtOAc = 3:1) to give known $24(53 \mathrm{mg}, 0.21 \mathrm{mmol}, 71 \%)$ as a colorless oil. ${ }^{1}$ H NMR (400

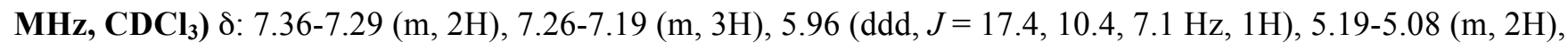

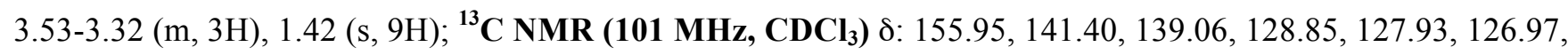
116.50, 49.95, 45.03, 28.52, and 27.56; HPLC analysis (OD-H, Hexanes: $i \mathrm{PrOH}=99: 1,1 \mathrm{~mL} / \mathrm{min})$ indicated $92 \%$ ee: $\mathrm{t}_{\mathrm{R}}($ major $)=18.8 \mathrm{~min}, \mathrm{t}_{\mathrm{R}}($ minor $)=17.5 \mathrm{~min} ;[\alpha]_{\mathbf{D}}{ }^{13}=29.9\left(\mathrm{c}=0.1, \mathrm{CHCl}_{3}\right)$. 


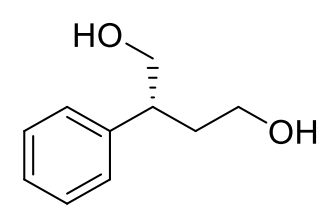

(R)-2-phenylbutane-1,4-diol (26). ${ }^{12}$ To a solution of $17(0.13 \mathrm{~g}, 0.50 \mathrm{mmol})$ in THF (1 mL) was added 9-BBN $(0.5 \mathrm{M}$ in hexanes, $1.5 \mathrm{~mL})$ dropwise under $\mathrm{N}_{2}$ atomsphere. The mixture was stirred at room temperature until all starting material was consumed (ca. $4 \mathrm{~h}$ ), as indicated by TLC. Then, $3 \mathrm{M} \mathrm{NaOH}(1.0 \mathrm{~mL})$ and $30 \% \mathrm{H}_{2} \mathrm{O}_{2}(0.9 \mathrm{~mL})$ was added, and the mixture was heated at $50{ }^{\circ} \mathrm{C}$ for $1.5 \mathrm{~h}$. After cooling to room temperature, the reaction was quenched by saturated aq. $\mathrm{Na}_{2} \mathrm{~S}_{2} \mathrm{O}_{3}(2.0 \mathrm{~mL})$. The mixture was extracted with EtOAc $(3 \mathrm{X} 15 \mathrm{~mL})$. The combined organic layer was washed with brine, dried $\left(\mathrm{Na}_{2} \mathrm{SO}_{4}\right)$, and concentrated. The residue was purified by column chromatography (20:1 DCM/MeOH) to afford known compound 26 as a colorless oil (61 mg, 74\% yield). ${ }^{1} \mathbf{H}$ NMR (400 MHz, $\left.\mathbf{C D C l}_{3}\right)$ 8: 7.37-7.29 (m, 2H), 7.27-7.20 (m, 3H), $3.78(\mathrm{~d}, J=6.7 \mathrm{~Hz}, 2 \mathrm{H}), 3.72-3.67(\mathrm{~m}, 1 \mathrm{H})$, 3.61-3.55 (m, 1H), 2.96 (dddd, $J=6.6,6.6,6.6,6.6 \mathrm{~Hz}, 1 \mathrm{H}), 2.07-1.97(\mathrm{~m}, 1 \mathrm{H}), 1.94-1.85(\mathrm{~m}, 1 \mathrm{H}) ;{ }^{13} \mathbf{C}$ NMR

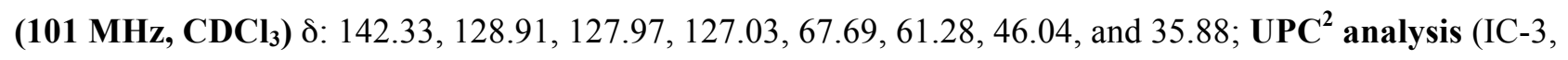
supercritical $\mathrm{CO}_{2}: i \mathrm{PrOH}=80: 20,2.0 \mathrm{~mL} / \mathrm{min}$ ) indicated 93\% ee: $\mathrm{t}_{\mathrm{R}}$ (major) $=2.7 \mathrm{~min}, \mathrm{t}_{\mathrm{R}}($ minor $)=2.3 \mathrm{~min}$; $[\alpha]_{\mathbf{D}}{ }^{13}=-40.6\left(\mathrm{c}=0.067, \mathrm{CHCl}_{3}\right)$. 


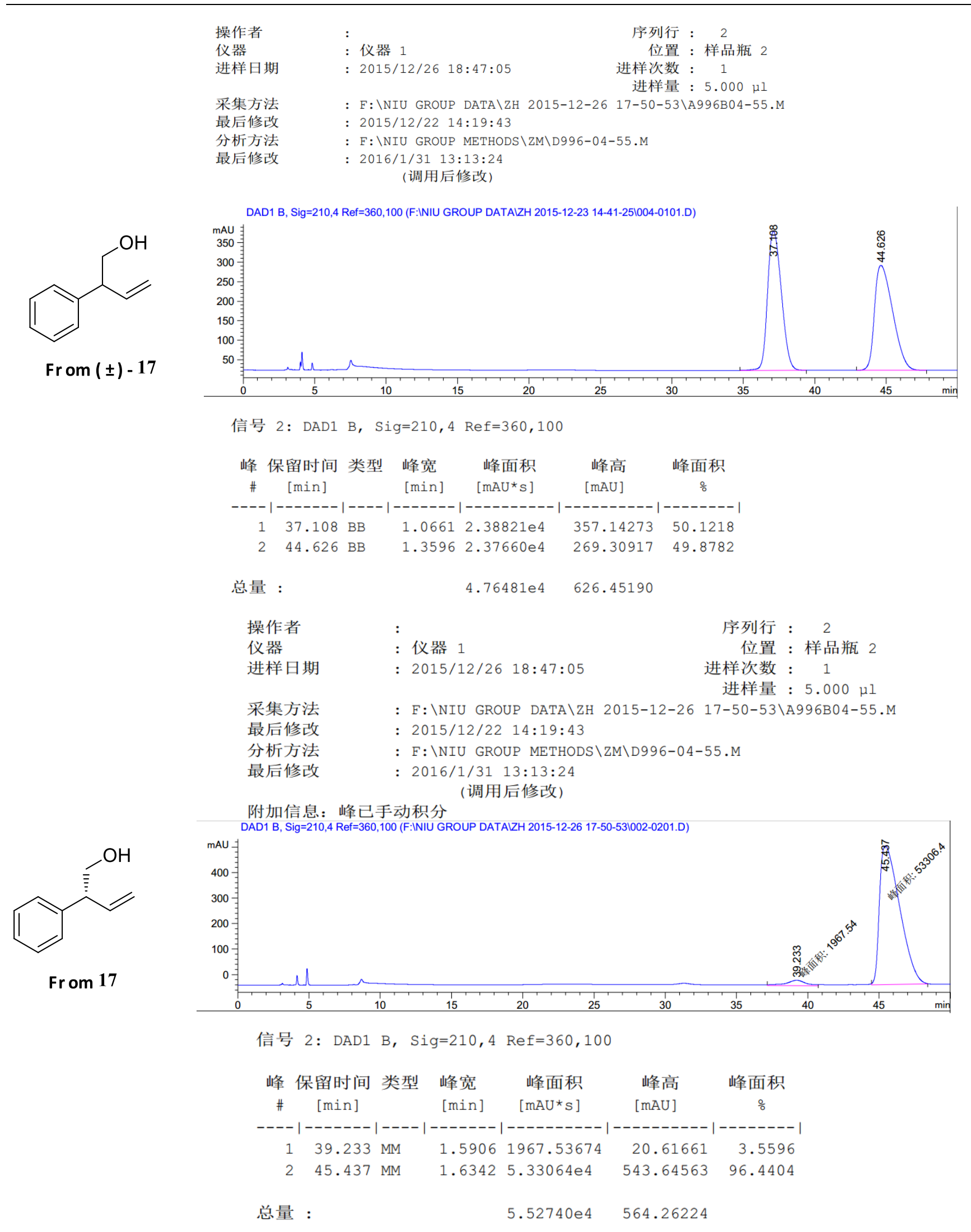


Homoallylic Boronic Esters

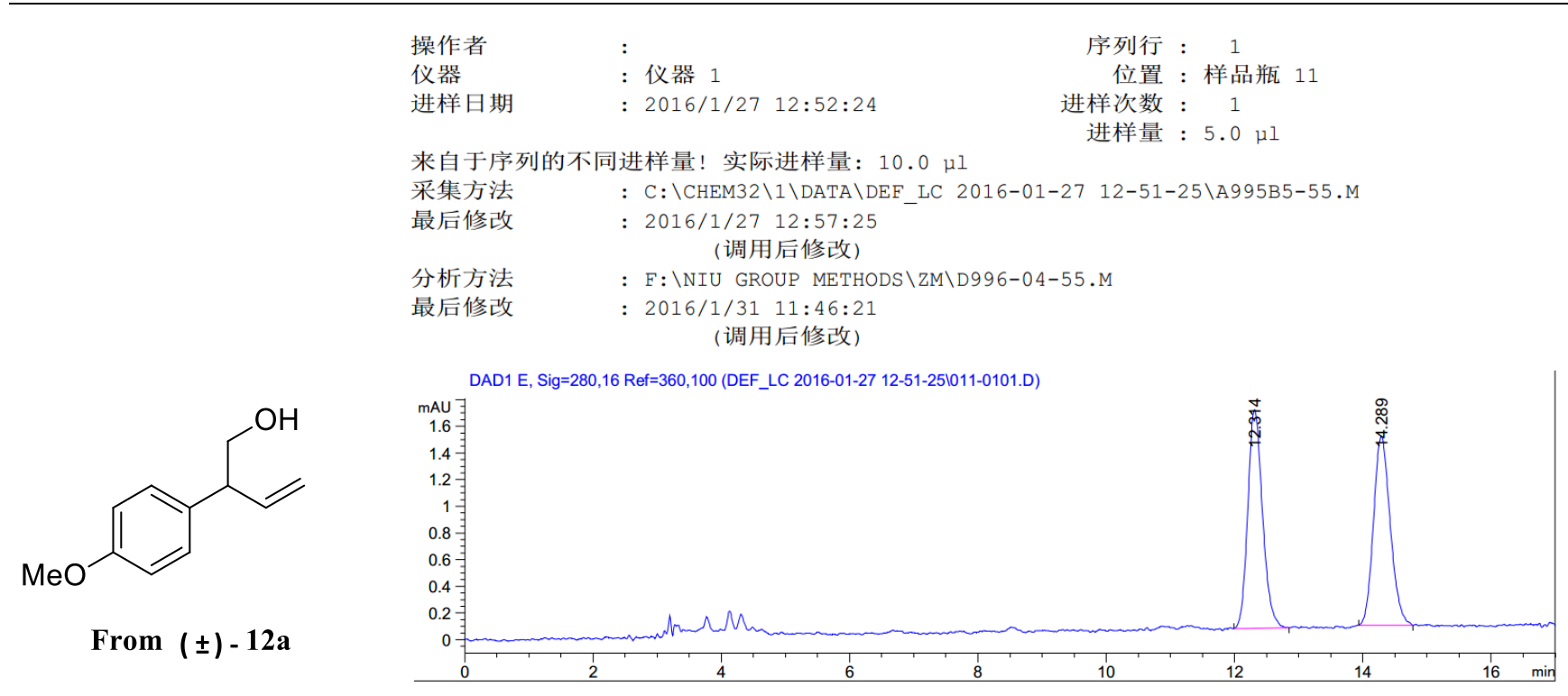

信号 5: DAD1 E, Sig=280,16 Ref $=360,100$

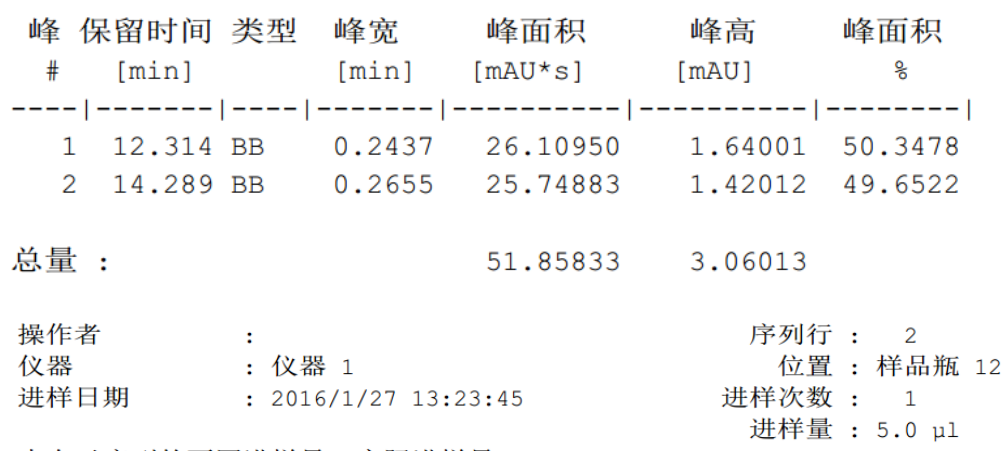

来自于序列的不同进样量! 实际进样量： $10.0 \mu \mathrm{l}$

采集方法 : C : \CHEM32\1\DATA \DEF_LC 2016-01-27 12-51-25\A995B5-55.M

最后修改 ：2016/1/27 13:47:41

(调用后修改)

分析方法 $\quad:$ F $: \backslash N I U$ GROUP METHODS $\backslash Z M \backslash D 996-04-55 . M$

最后修改 : 2016/1/31 11:46:21

(调用后修改)<smiles>C=C[C@@H](CO)c1ccc(OC)cc1</smiles>

From 12a

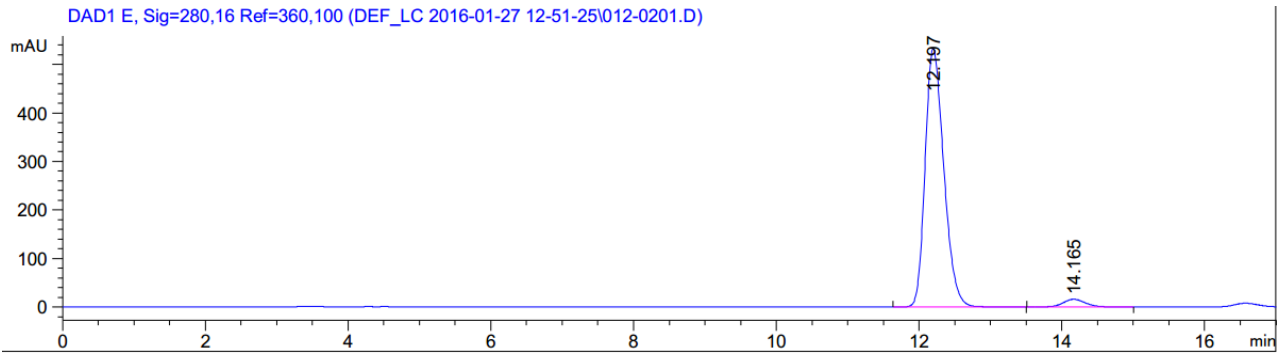

信号 5: DAD1 E， Sig=280,16 Ref=360,100

\begin{tabular}{|c|c|c|c|c|c|c|}
\hline $\begin{array}{r}\text { 峰 } \\
\text { \# }\end{array}$ & $\begin{array}{c}\text { 保留时间 } \\
\text { [min] }\end{array}$ & 类型 & $\begin{array}{l}\text { 峰宽 } \\
\text { [min] }\end{array}$ & $\begin{array}{l}\text { 峰面积 } \\
{\left[\mathrm{mAU}{ }^{*} \mathrm{~S}\right]}\end{array}$ & $\begin{array}{l}\text { 峰高 } \\
\text { [mAU] }\end{array}$ & $\begin{array}{c}\text { 峰面积 } \\
\text { 。 }\end{array}$ \\
\hline & 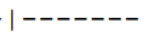 & & - & --- & --- & \\
\hline 1 & 12.197 & BB & 0.2824 & 9690.23730 & 532.36285 & 96.5 \\
\hline 2 & 14. & סי & 330 & 344.06680 & 15.86533 & 3.4 \\
\hline
\end{tabular}

总量 :

$1.00343 e 4 \quad 548.22819$ 
Homoallylic Boronic Esters

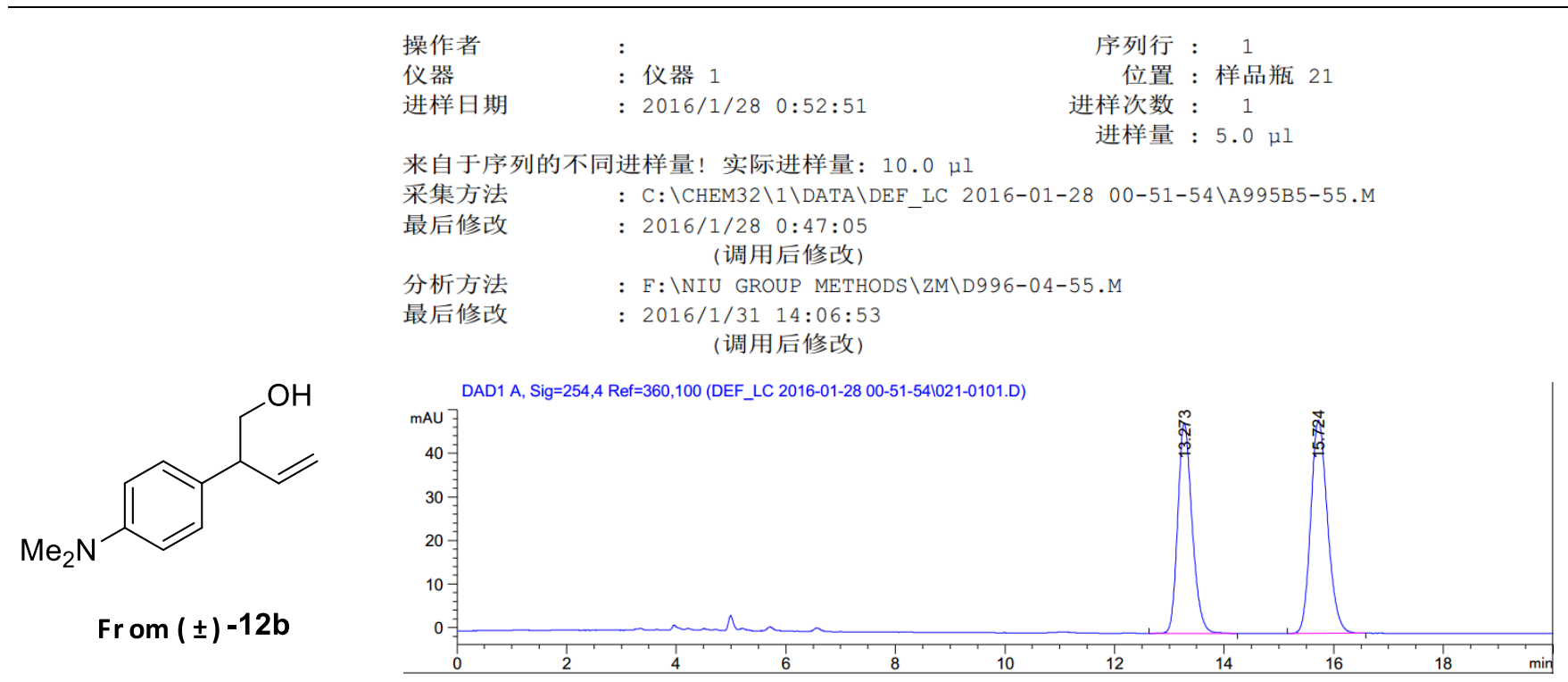

信号 1: DAD1 A, Sig=254,4 Ref=360,100

\begin{tabular}{|c|c|c|c|c|c|c|}
\hline $\begin{array}{r}\text { 峰 } \\
\text { \# }\end{array}$ & $\begin{array}{c}\text { 保留时间 } \\
\text { [min] }\end{array}$ & 类型 & $\begin{array}{l}\text { 峰宽 } \\
\text { [min] }\end{array}$ & $\begin{array}{l}\text { 峰面积 } \\
{\left[\mathrm{MAU}^{*} \mathrm{~S}\right]}\end{array}$ & $\begin{array}{l}\text { 峰高 } \\
\text { [mAU] }\end{array}$ & $\begin{array}{c}\text { 峰面积 } \\
\frac{\circ}{0}\end{array}$ \\
\hline & & & -0 & ----- & --- & \\
\hline & 13. & & 0.2811 & 881.16 & 48. & 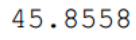 \\
\hline & 15 & & 0.3279 & 1040. & 917 & 54.14 \\
\hline
\end{tabular}

总量：

$1921.60718 \quad 97.21468$

操作者

仪器

进样日期

采集方法

最后修改

分析方法

最后修改
: 仪器 1

: 2016/1/28 1:24:06
序列行 : 2

位置：样品瓶 22

进样次数 : 1

进样量 : $5.0 \mu \mathrm{I}$

: C: \CHEM32\1\DATA \DEF_LC 2016-01-28 00-51-54\A995B5-55.M : 2016/1/28 0:47:05

(调用后修改)

F:\NIU GROUP METHODS $\backslash Z M \backslash D 996-04-55 . M$

: 2016/1/31 14:06:53

(调用后修改)<smiles>C=C[C@H](CO)c1ccc(N(C)C)cc1</smiles>

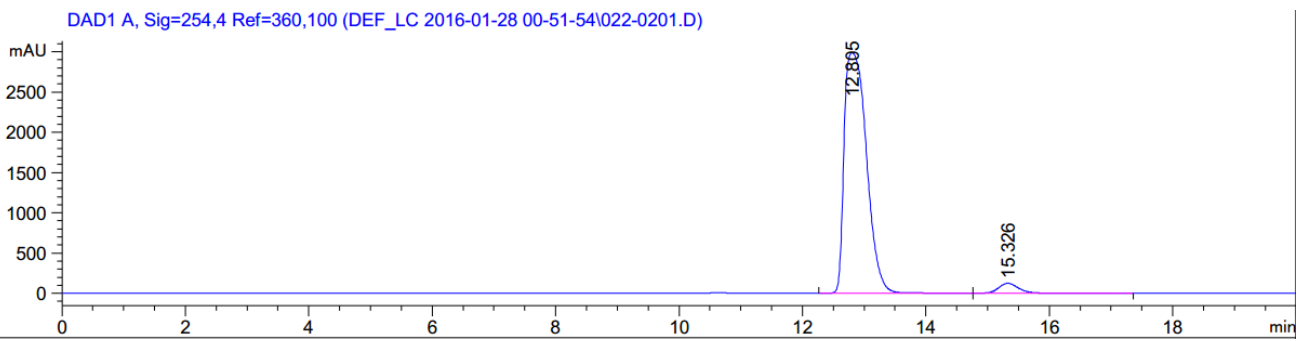

信号 1: DAD1 A， Sig=254,4 Ref=360,100

\begin{tabular}{|c|c|c|c|c|c|c|}
\hline $\begin{array}{r}\text { 峰 } \\
\#\end{array}$ & $\begin{array}{c}\text { 保留时间 } \\
\text { [min] }\end{array}$ & 类型 & $\begin{array}{l}\text { 峰宽 } \\
\text { [min] }\end{array}$ & $\begin{array}{c}\text { 峰面积 } \\
{\left[m A U^{*} \mathrm{~S}\right]}\end{array}$ & $\begin{array}{l}\text { 峰高 } \\
\text { [mAU] }\end{array}$ & $\begin{array}{c}\text { 峰面积 } \\
\text { 。 }\end{array}$ \\
\hline & & & & -- & --- & \\
\hline 1 & & ס & & $7.48791 \mathrm{e} 4$ & 2986.48340 & 190 \\
\hline ? & 15.326 & B & 92 & 2700.51196 & 122.51337 & 3.4810 \\
\hline
\end{tabular}

总量 :

$7.75796 \mathrm{e} 4 \quad 3108.99677$ 


\section{Homoallylic Boronic Esters}

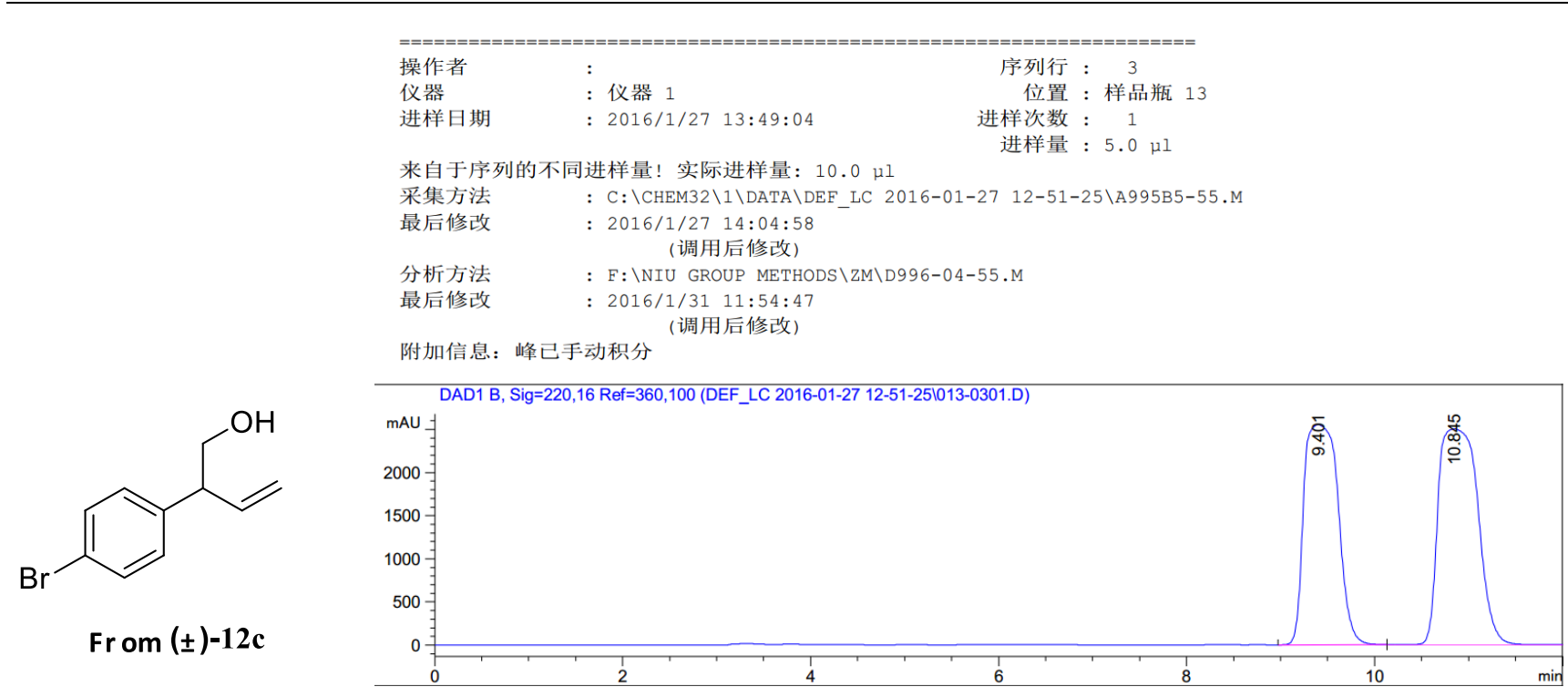

信号 2: DAD1 B, Sig=220,16 Ref=360,100

\begin{tabular}{|c|c|c|c|c|c|c|}
\hline $\begin{array}{r}\text { 峰 } \\
\text { \# }\end{array}$ & $\begin{array}{c}\text { 保留时间 } \\
\text { [min] }\end{array}$ & 类型 & $\begin{array}{l}\text { 峰宽 } \\
\text { [min] }\end{array}$ & $\begin{array}{l}\text { 峰面积 } \\
{\left[\mathrm{mAU}{ }^{\star} \mathrm{S}\right]}\end{array}$ & $\begin{array}{l}\text { 峰高 } \\
\text { [mAU] }\end{array}$ & $\begin{array}{c}\text { 峰面积 } \\
\text { 。 }\end{array}$ \\
\hline- & -- & & ---1 & | --------- & $|----------|$ & --- \\
\hline 1 & 9.401 & $\mathrm{BB}$ & 0.4091 & $6.40900 \mathrm{e} 4$ & 2551.02393 & 46.8763 \\
\hline 2 & 10.845 & $\mathrm{BB}$ & 0.4727 & $7.26315 \mathrm{e} 4$ & 2501.68750 & 53.1237 \\
\hline 总量 & : & & & 1.367 & 5052 & \\
\hline
\end{tabular}

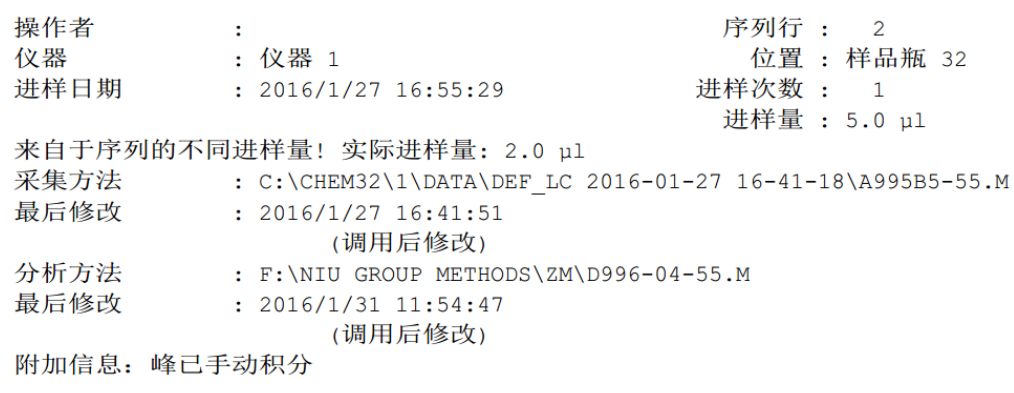<smiles>C=C[C@H](CO)c1ccc(Br)cc1</smiles>

From 12c

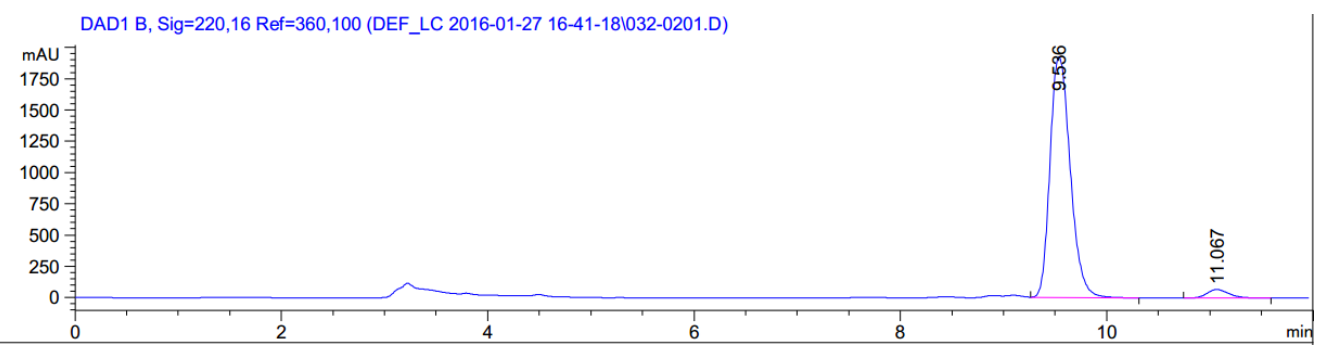

信号 2: DAD1 B, Sig=220,16 Ref=360,100

\begin{tabular}{|c|c|c|c|c|c|c|}
\hline $\begin{array}{r}\text { 峰 } \\
\text { \# }\end{array}$ & $\begin{array}{c}\text { 保留时间 } \\
\text { [min] }\end{array}$ & 类型 & $\begin{array}{l}\text { 峰宽 } \\
\text { [min] }\end{array}$ & $\begin{array}{l}\text { 峰面积 } \\
{\left[m A U^{\star} s\right]}\end{array}$ & $\begin{array}{l}\text { 峰高 } \\
\text { [mAU] }\end{array}$ & $\begin{array}{c}\text { 峰面积 } \\
\text { 。 }\end{array}$ \\
\hline- & $\mid-------$ & -- & -------1 & |---------- & $|----------|$ & $--------\mid$ \\
\hline 1 & 9.536 & $\mathrm{BB}$ & 0.2128 & $2.61955 \mathrm{e} 4$ & 1923.99585 & 96.3360 \\
\hline 2 & 11.067 & $\mathrm{BB}$ & 0.2245 & 996.31268 & 68.18327 & 3.6640 \\
\hline 总量 & 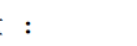 & & & $2.71918 \mathrm{e} 4$ & 1992.17912 & \\
\hline
\end{tabular}


Homoallylic Boronic Esters

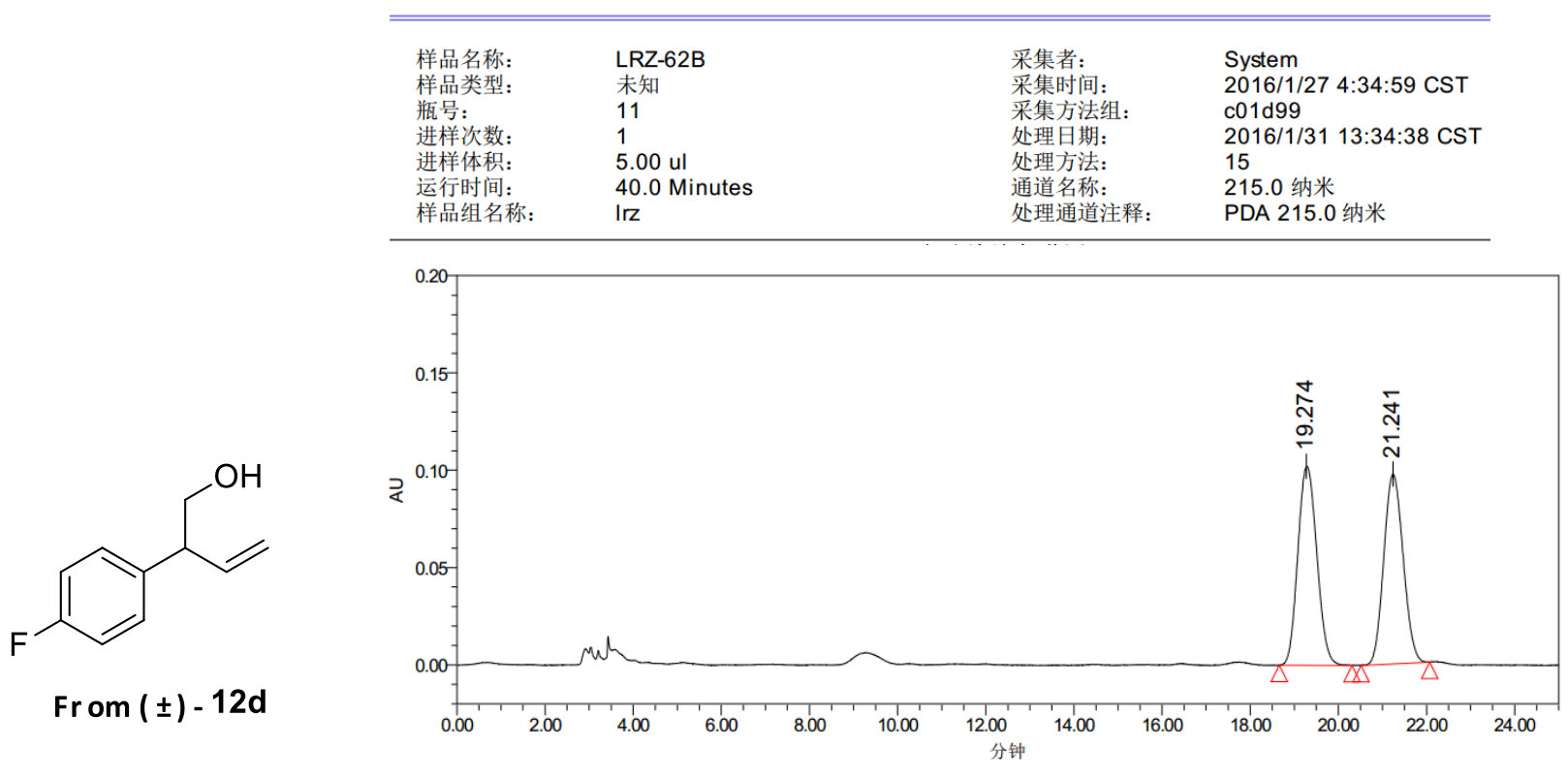

处理通道: PDA 215.0 纳米

\begin{tabular}{|c|c|c|c|r|c|}
\hline & 处理通道 & $\begin{array}{c}\text { 保留时间 } \\
\text { (分钟) }\end{array}$ & 面积 & \% 面积 & 峰高 \\
\hline 1 & PDA 215.0 纳米 & 19.274 & 3059457 & 50.63 & 102284 \\
\hline 2 & PDA 215.0 纳米 & 21.241 & 2983556 & 49.37 & 97559 \\
\hline
\end{tabular}

\begin{tabular}{llll}
\hline \hline & & & \\
样品名称: & ZM264 & 采集者: & System \\
样品类型: & 未知 & 采集时间: & $2016 / 1 / 275: 15: 37$ CST \\
瓶号: & 12 & 采集方法组: & c01d99 \\
进样次数: & 1 & 处理日期: & $2016 / 1 / 3113: 32: 18$ CST \\
进样体积: & 5.00 ul & 处理方法: & 14 \\
运行时间: & 40.0 Minutes & 通道名称: & 215.0 纳米 \\
样品组名称: & Irz & 处理通道注释: & PDA 215.0 纳米 \\
\hline
\end{tabular}<smiles>C=C[C@@H](CO)c1ccc(F)cc1</smiles>

From 12d

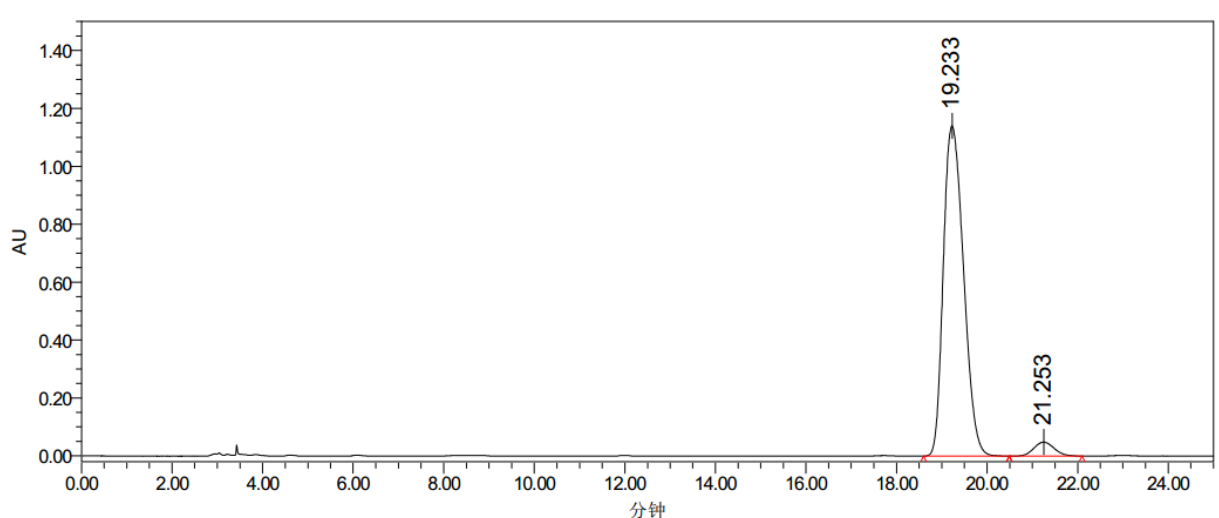

处理通道: PDA 215.0 纳米

\begin{tabular}{|r|c|r|r|r|r|}
\hline & 处理通道 & \multicolumn{1}{|c|}{$\begin{array}{c}\text { 保留时间 } \\
\text { (分钟) }\end{array}$} & 面积 & \% 面积 & 峰高 \\
\hline 1 & PDA 215.0 纳米 & 19.233 & 35272073 & 95.94 & 1140717 \\
\hline 2 & PDA 215.0 纳米 & 21.253 & 1491778 & 4.06 & 48031 \\
\hline
\end{tabular}


Homoallylic Boronic Esters

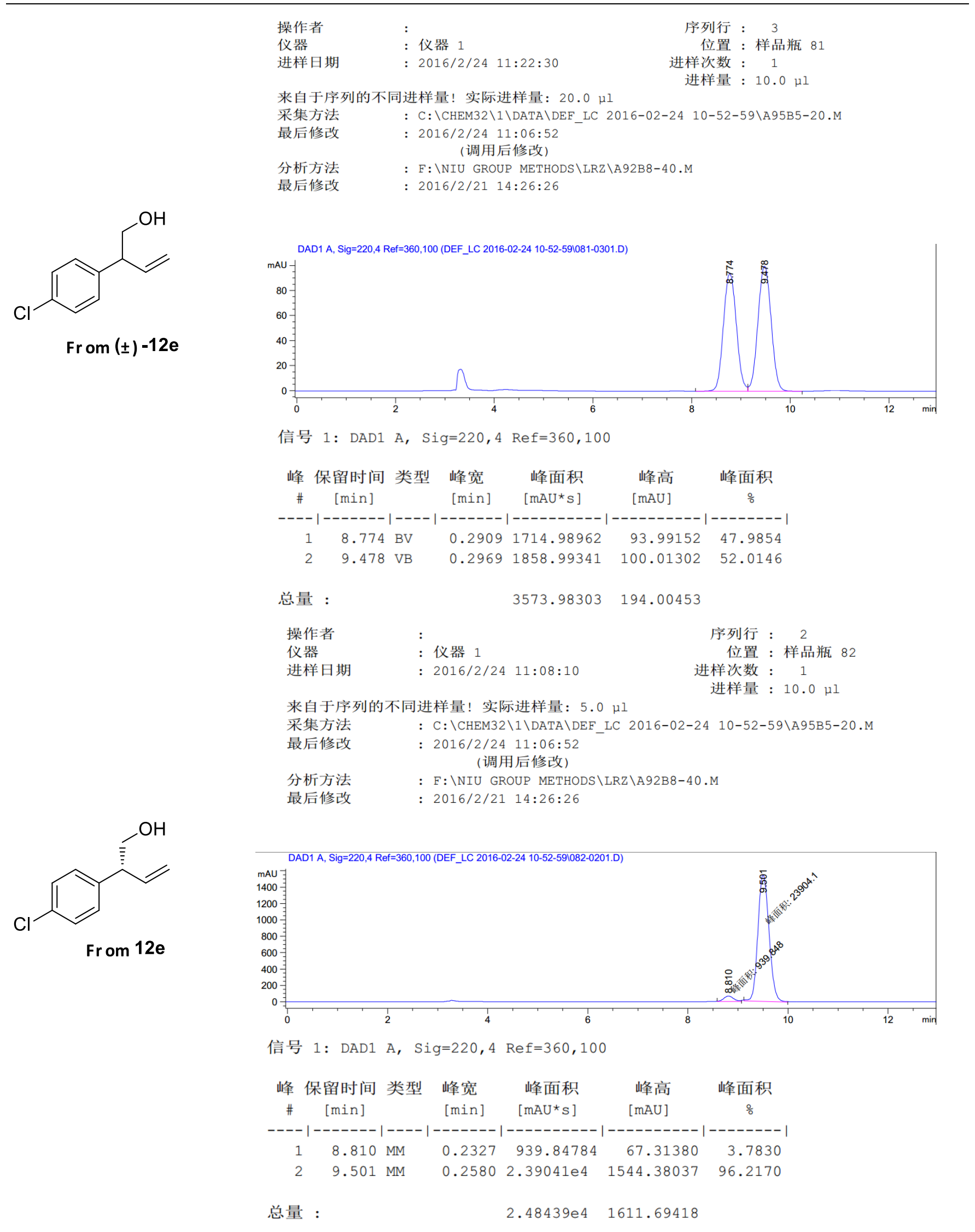


<smiles>C=CC(CO)c1ccccc1C</smiles>

From $( \pm)-12 f$<smiles>C=CC(CO)c1ccccc1C</smiles>

From $12 f$

\begin{tabular}{llll}
\hline \hline & & & \\
样品名称: & LRZ-66A-2 & 采集者: & System \\
样品类型: & 标准样 & 采集时间: & $2016 / 4 / 161: 13: 21$ CST \\
瓶号: & 70 & 采集方法组: & C004D996L \\
进样次数: & 1 & 处理日期: & $2016 / 4 / 2123: 13: 02$ CST \\
进样体积: & $5.00 \mathrm{ul}$ & 处理方法: & Default \\
运行时间: & 180.0 Minutes & 通道名称: & 210.0 纳米 \\
样品组名称: & $I r z$ & 处理通道注释: & PDA 210.0 纳米
\end{tabular}

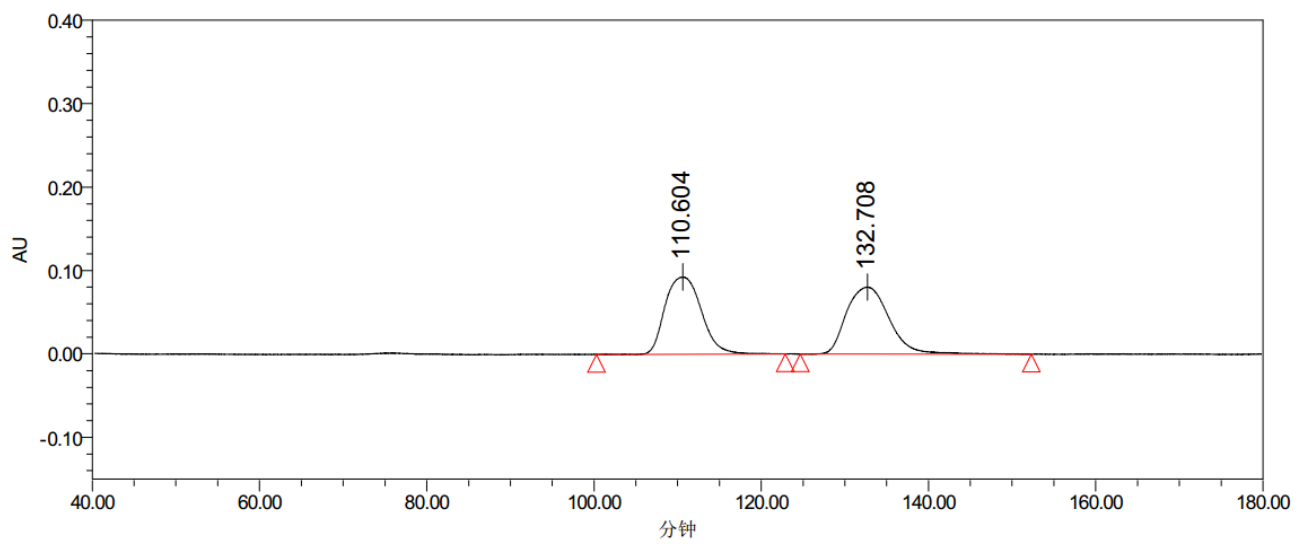

处理通道: PDA 210.0 纳米

\begin{tabular}{|c|c|c|c|r|r|}
\hline & 处理通道 & $\begin{array}{c}\text { 保留时间 } \\
\text { (分钟) }\end{array}$ & 面积 & \% 面积 & 峰高 \\
\hline 1 & PDA 210.0 纳米 & 110.604 & 28105945 & 49.57 & 92747 \\
\hline 2 & PDA 210.0 纳米 & 132.708 & 28592103 & 50.43 & 80582 \\
\hline
\end{tabular}

\begin{tabular}{|c|c|c|c|}
\hline 样品名称: & $38 b-1$ & 采集者: & System \\
\hline 样品类型: & 标准样 & 采集时间: & 2016/4/15 22:12:27 CST \\
\hline 瓶号: & 71 & 采集方法组: & C004D996L \\
\hline 进样次数: & 1 & 处理日期: & 2016/4/21 23:16:59 CST \\
\hline 进样体积: & $5.00 \mathrm{ul}$ & 处理方法: & 1234 \\
\hline 运行时间: & 180.0 Minutes & 通道名称: & 209.9 纳米 \\
\hline 样品组名称: & Irz & 处理通道注释: & PDA 209.9 纳米 \\
\hline
\end{tabular}

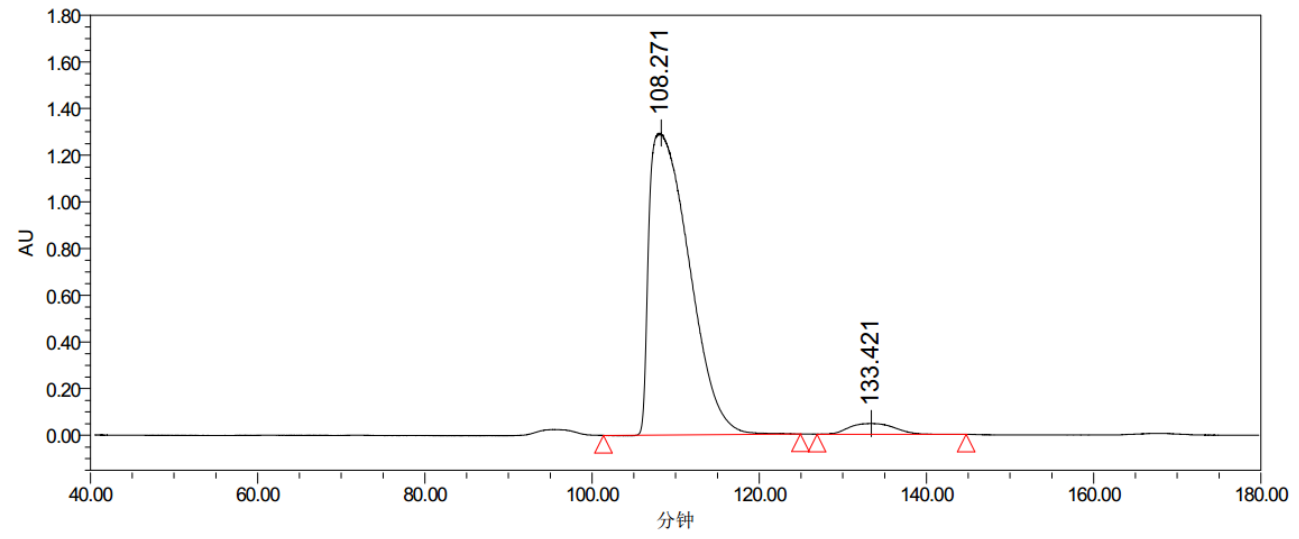

处理通道: PDA 209.9 纳米

\begin{tabular}{|r|c|c|r|r|r|}
\hline & 处理通道 & $\begin{array}{c}\text { 保留时间 } \\
\text { (分钟) }\end{array}$ & 面积 & \% 面积 & 峰高 \\
\hline 1 & PDA 209.9 纳米 & 108.271 & 433035887 & 96.13 & 1294735 \\
\hline 2 & PDA 209.9 纳米 & 133.421 & 17411839 & 3.87 & 46272 \\
\hline
\end{tabular}


Homoallylic Boronic Esters

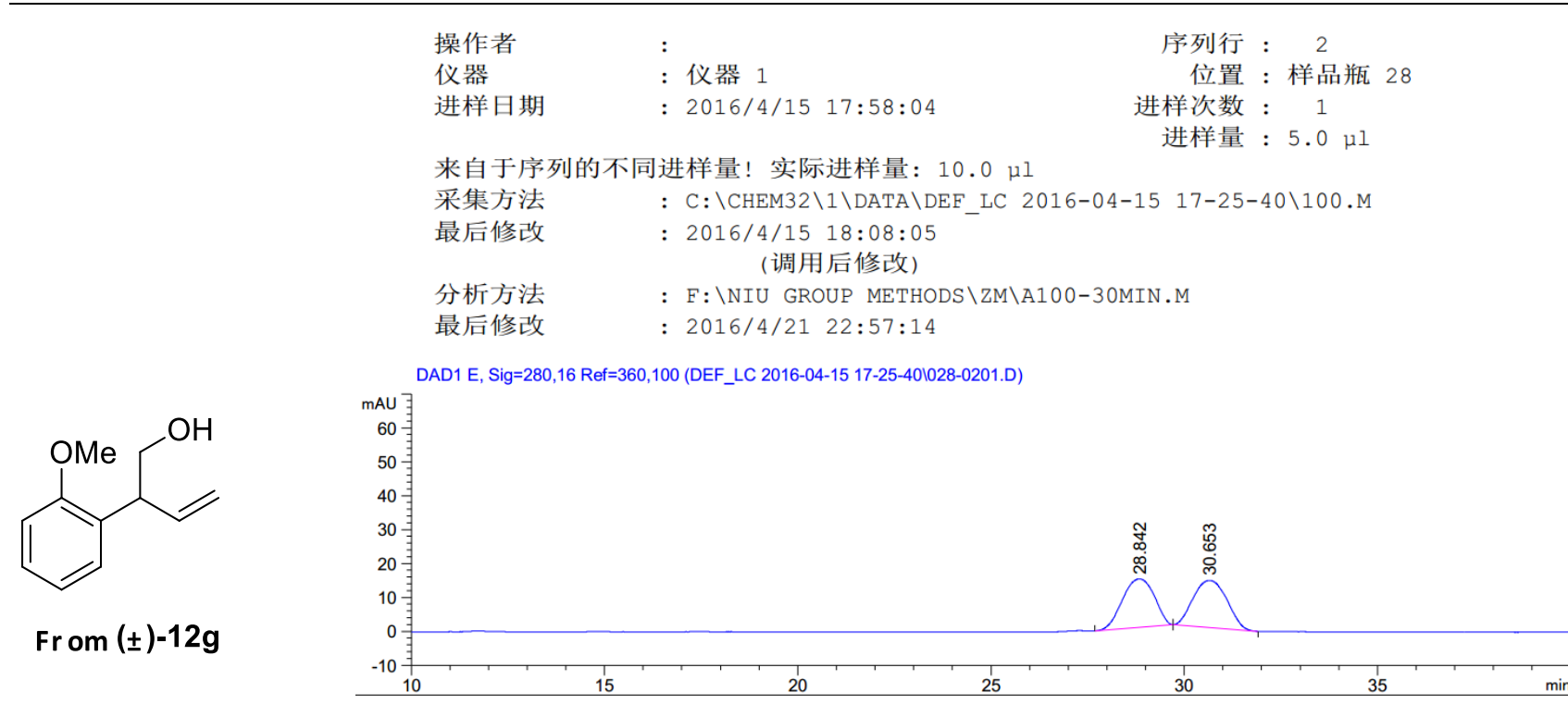

信号 5: DAD1 E, Sig=280,16 Ref=360,100

\begin{tabular}{|c|c|c|c|c|c|c|}
\hline $\begin{array}{r}\text { 峰 } \\
\text { \# }\end{array}$ & $\begin{array}{c}\text { 保留时间 } \\
\text { [min] }\end{array}$ & 类型 & $\begin{array}{l}\text { 峰宽 } \\
\text { [min] }\end{array}$ & $\begin{array}{l}\text { 峰面积 } \\
{\left[\mathrm{mAU}^{*} \mathrm{~S}\right]}\end{array}$ & $\begin{array}{c}\text { 峰高 } \\
\text { [mAU] }\end{array}$ & $\begin{array}{c}\text { 峰面积 } \\
\text { 。 }\end{array}$ \\
\hline- & -- & & 1 & ---------- & ---- & . \\
\hline 1 & 28.842 & $\mathrm{BB}$ & 0.8593 & 819.01581 & 14.27663 & 49.2527 \\
\hline 2 & 30.653 & $\mathrm{BB}$ & 0.8060 & 843.87067 & 13.90514 & 50.7473 \\
\hline
\end{tabular}

总量： $\quad 1662.88647 \quad 28.18177$

$\begin{array}{llr}\text { 操作者 } & : & \text { 序列行 : } \\ \text { 仪器 } & \text { : 仪器 } 1 & \text { 位置 : 样品瓶 } 29 \\ \text { 进样日期 } & : \text { 2016/4/15 } 18: 49: 20 & \text { 进样次数 : } 1 \\ & & \text { 进样量 : } 5.0 \mu 1\end{array}$

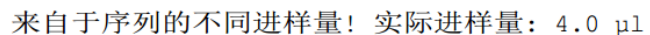

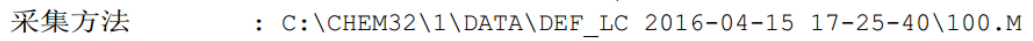

最后修改 : 2016/4/15 18:08:05 (调用后修改)

分析方法 $:$ F $: \backslash N I U$ GROUP METHODS $\backslash Z M \backslash A 100-30 M I N . M$

最后修改 : 2016/4/21 22:46:33

(OMe

From 12g
DAD1 E, Sig=280,16 Ref=360,100 (DEF LC 2016-04-15 17-25-401029-0301.D)

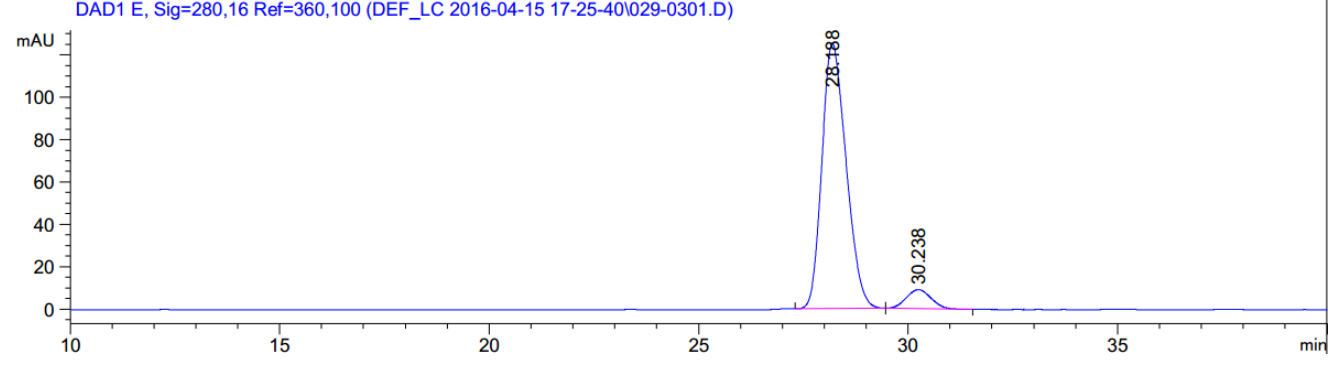

信号 5: DAD1 E, Sig=280,16 Ref=360,100

\begin{tabular}{|c|c|c|c|c|c|c|}
\hline $\begin{array}{r}\text { 峰 } \\
\text { \# }\end{array}$ & $\begin{array}{c}\text { 保留时间 } \\
\text { [min] }\end{array}$ & 类型 & $\begin{array}{l}\text { 峰宽 } \\
\text { [min] }\end{array}$ & $\begin{array}{l}\text { 峰面积 } \\
{\left[\mathrm{mAU}^{*} \mathrm{~S}\right]}\end{array}$ & $\begin{array}{l}\text { 峰高 } \\
\text { [mAU] }\end{array}$ & $\begin{array}{c}\text { 峰面积 } \\
\text { 。 }\end{array}$ \\
\hline & & & ------ & $|----------|$ & ---------- & \\
\hline 1 & 28.188 & $\mathrm{BB}$ & 0.6211 & 5020.29541 & 125.38090 & 93.0855 \\
\hline 2 & 30.238 & $\mathrm{BB}$ & 0.6313 & 372.91107 & 9.00275 & 6.9145 \\
\hline
\end{tabular}

总量 :

$5393.20648 \quad 134.38365$ 
Homoallylic Boronic Esters

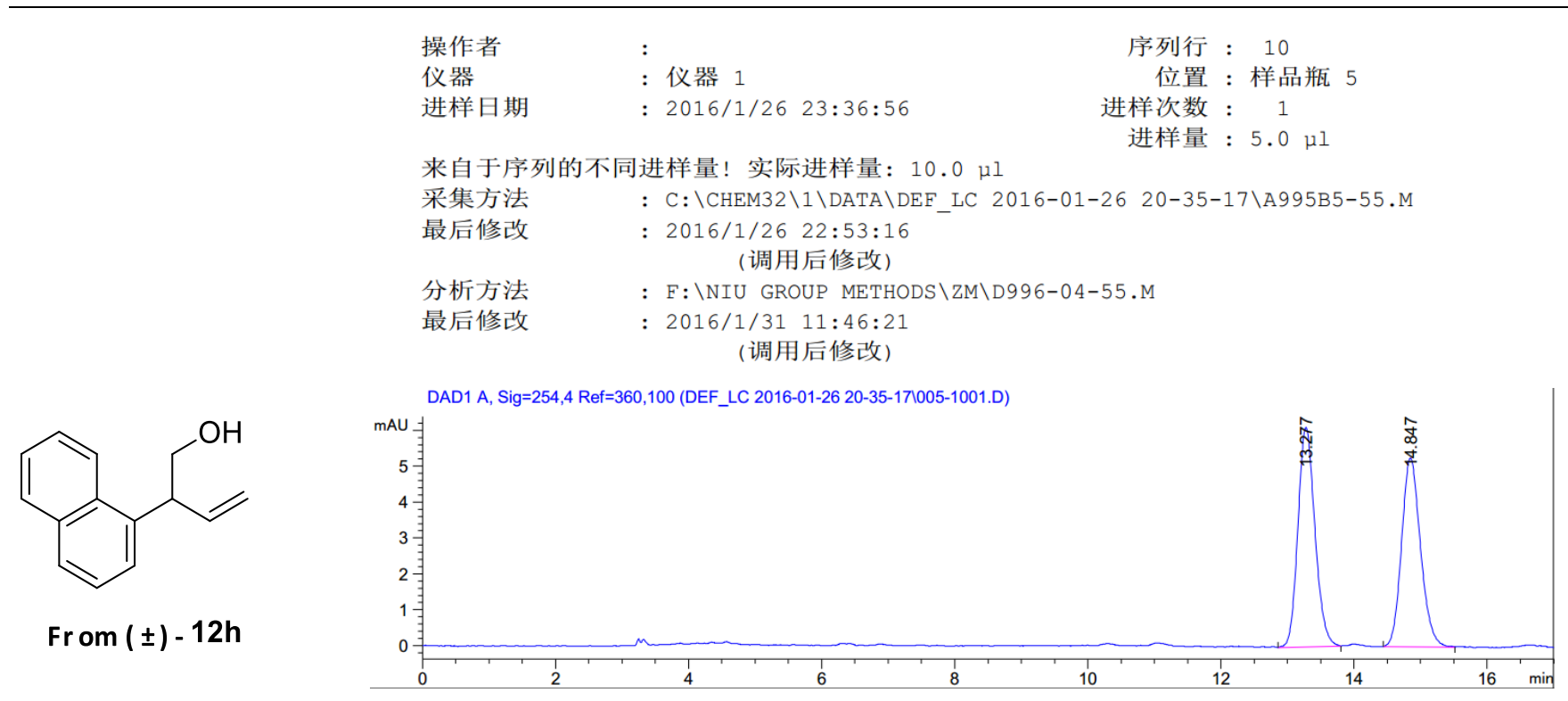

信号 1: DAD1 A， Sig=254,4 Ref=360,100

\begin{tabular}{|c|c|c|c|c|c|c|}
\hline $\begin{array}{r}\text { 峰 } \\
\text { \# }\end{array}$ & $\begin{array}{c}\text { 保留时间 } \\
\text { [min] }\end{array}$ & 类型 & $\begin{array}{l}\text { 峰宽 } \\
\text { [min] }\end{array}$ & $\begin{array}{l}\text { 峰面积 } \\
{\left[m A{ }^{*} s\right]}\end{array}$ & $\begin{array}{l}\text { 峰高 } \\
\text { [mAU] }\end{array}$ & $\begin{array}{c}\text { 峰面积 } \\
\text { 。 }\end{array}$ \\
\hline & & & -- & ------- & -- & ---1 \\
\hline 1 & 13.27 & $3 \mathrm{~B}$ & 0.2726 & 108.38966 & 6.12024 & 261 \\
\hline 2 & 14.847 & & 015 & 103.61510 & 5.26655 & 48.8739 \\
\hline
\end{tabular}

总量： $\quad 212.00476 \quad 11.38679$

$\begin{array}{llr}\text { 操作者 } & : & \text { 序列行 }: 8 \\ \text { 仪器 } & : \text { 仪器 } 1 & \text { 位置 }: \text { 样品瓶 } 8 \\ \text { 进样日期 } & : 2016 / 1 / 26 \quad 22: 54: 18 & \text { 进样次数 }: 1 \\ & & \text { 进样量 }: 5.0 \mu 1\end{array}$

来自于序列的不同进样量! 实际进样量： $10.0 \mu 1$

采集方法 ：C：\CHEM32\1\DATA \DEF_LC 2016-01-26 20-35-17\A995B5-55.M

最后修改 ：2016/1/26 22:53:16

分析方法 $\quad: \mathrm{F}: \backslash N I U$ GROUP METHODS $\backslash Z M \backslash D 996-04-55 . M$

最后修改 ：2016/1/31 11:46:21<smiles>C=C[C@H](CO)c1cccc2ccccc12</smiles>

From 12h
DAD1 A, Sig=254, 4 Ref=360, 100 (DEF LC 2016-01-26 20-35-171008-0801.D)

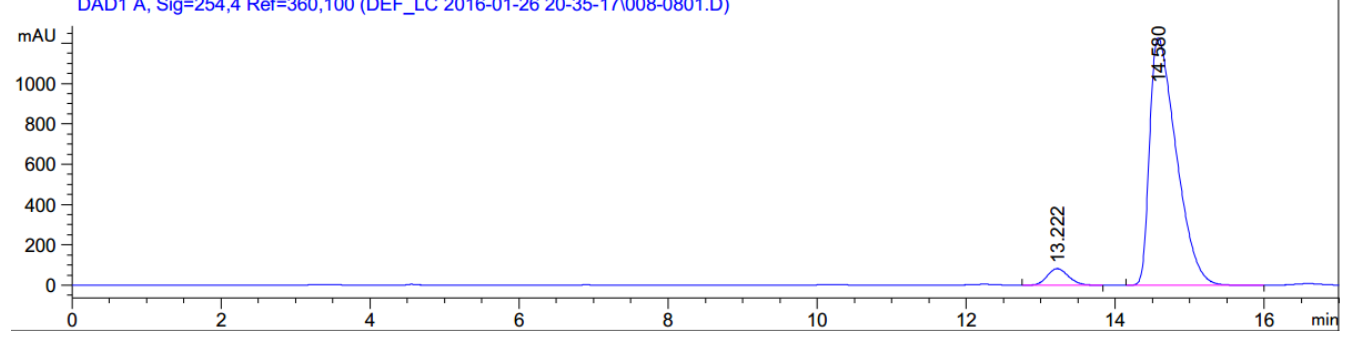

信号 1: DAD1 A, Sig=254,4 Ref=360,100

\begin{tabular}{|c|c|c|c|c|c|c|}
\hline $\begin{array}{r}\text { 峰 } \\
\#\end{array}$ & $\begin{array}{c}\text { 保留时间 } \\
\text { [min] }\end{array}$ & 类型 & $\begin{array}{l}\text { 峰宽 } \\
\text { [min] }\end{array}$ & $\begin{array}{c}\text { 峰面积 } \\
{\left[m A U^{\star} s\right]}\end{array}$ & $\begin{array}{l}\text { 峰高 } \\
\text { [mAU] }\end{array}$ & $\begin{array}{c}\text { 峰面积 } \\
\frac{\circ}{0}\end{array}$ \\
\hline- & ----- & & ------- & ---------- & ---------- & -------- \\
\hline 1 & 13.222 & BB & 0.3070 & 1628.41675 & 82.24026 & 5.0823 \\
\hline 2 & 14.580 & $\mathrm{BB}$ & 0.3853 & $3.04126 \mathrm{e} 4$ & 1226.25525 & 94.9177 \\
\hline 总量 & : & & & $3.20410 \mathrm{e} 4$ & 1308.49551 & \\
\hline
\end{tabular}


Homoallylic Boronic Esters

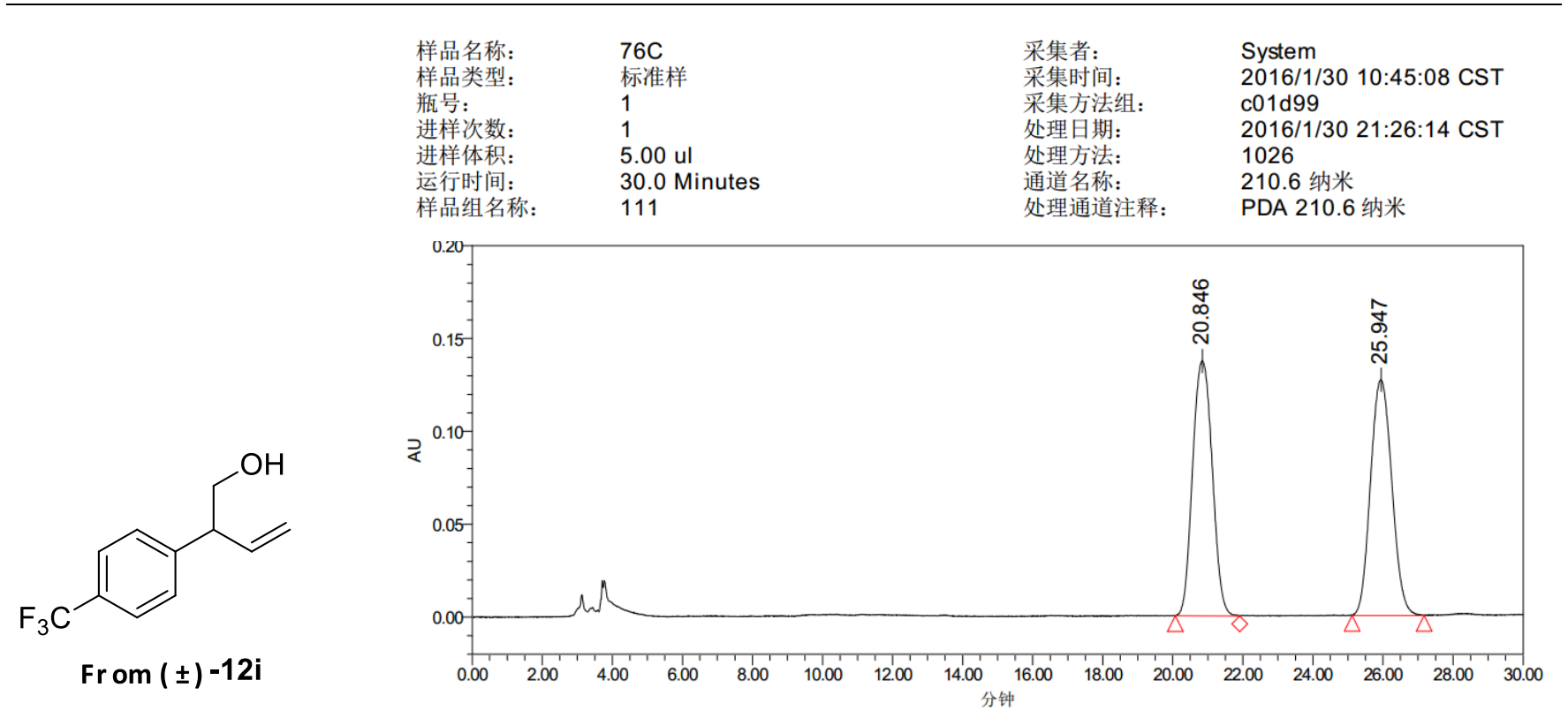

处理通道: PDA 210.6 纳米

\begin{tabular}{|l|c|c|c|r|r|}
\hline & 处理通道 & $\begin{array}{c}\text { 保留时间 } \\
\text { (分钟) }\end{array}$ & 面积 & \% 面积 & 峰高 \\
\hline 1 & PDA 210.6 纳米 & 20.846 & 5348906 & 49.96 & 137342 \\
\hline 2 & PDA 210.6 纳米 & 25.947 & 5357962 & 50.04 & 126998 \\
\hline
\end{tabular}

$\begin{array}{llll}\text { 样品名称: } & 295 & \text { 采集者: } & \text { System } \\ \text { 样品类型: } & \text { 标准样 } & \text { 采集时间: } & 2016 / 1 / 30 ~ 11: 15: 48 \text { CST } \\ \text { 瓶号: } & 2 & \text { 采集方法组: } & \text { c01d99 } \\ \text { 进样次数: } & 1 & \text { 处理日期: } & 2016 / 1 / 3021: 31: 40 \text { CST } \\ \text { 进样体积: } & 5.00 \mathrm{ul} & \text { 处理方法: } & 1 \\ \text { 运行时间: } & 30.0 \text { Minutes } & \text { 通道名称: } & 210.6 \text { 纳米 } \\ \text { 样品组名称: } & 111 & \text { 处理通道注释: } & \text { PDA 210.6 纳米 }\end{array}$<smiles>C=C[C@@H](CO)c1ccc(C(F)(F)F)cc1</smiles>

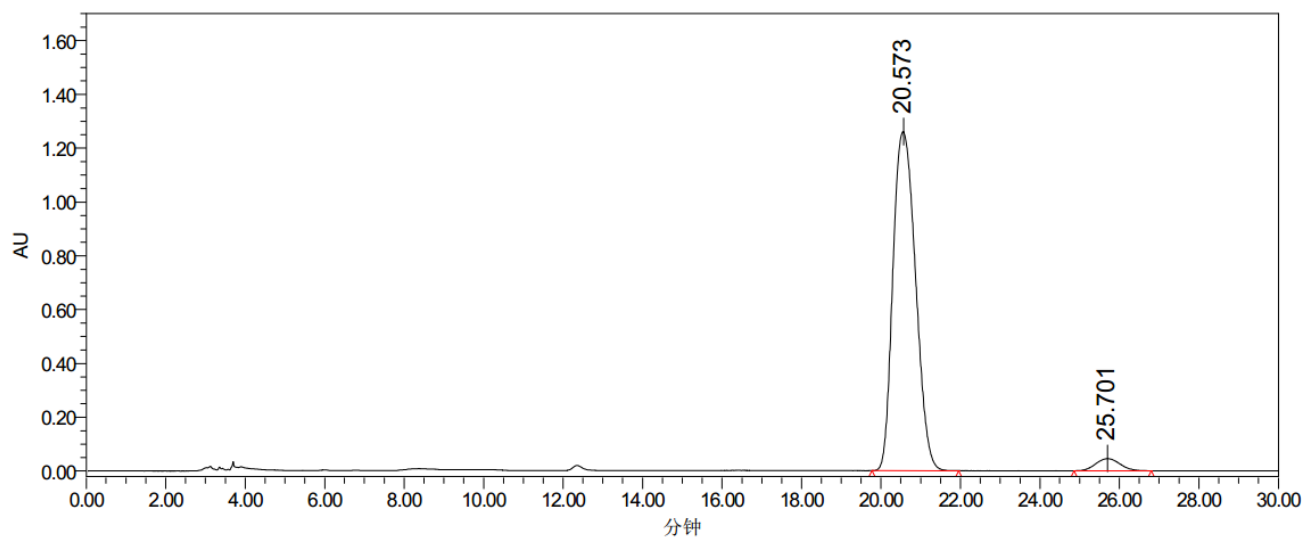

处理通道: PDA 210.6 纳米

\begin{tabular}{|r|c|r|r|r|r|}
\hline & 处理通道 & $\begin{array}{c}\text { 保留时间 } \\
\text { (分钟) }\end{array}$ & 面积 & \% 面积 & 峰高 \\
\hline 1 & PDA 210.6 纳米 & 20.573 & 49715534 & 96.28 & 1260845 \\
\hline 2 & PDA 210.6 纳米 & 25.701 & 1921831 & 3.72 & 45333 \\
\hline
\end{tabular}


Homoallylic Boronic Esters

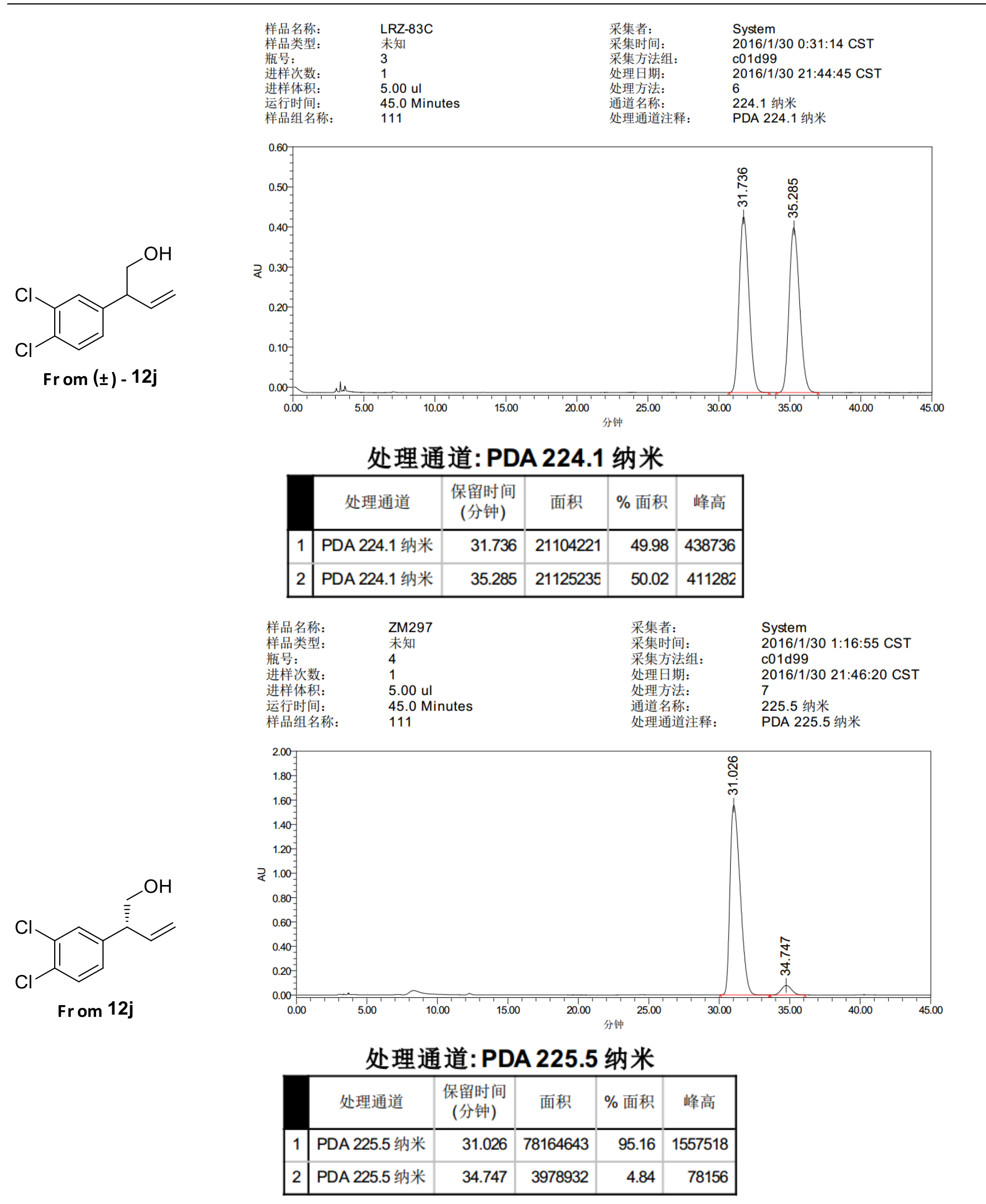


Homoallylic Boronic Esters

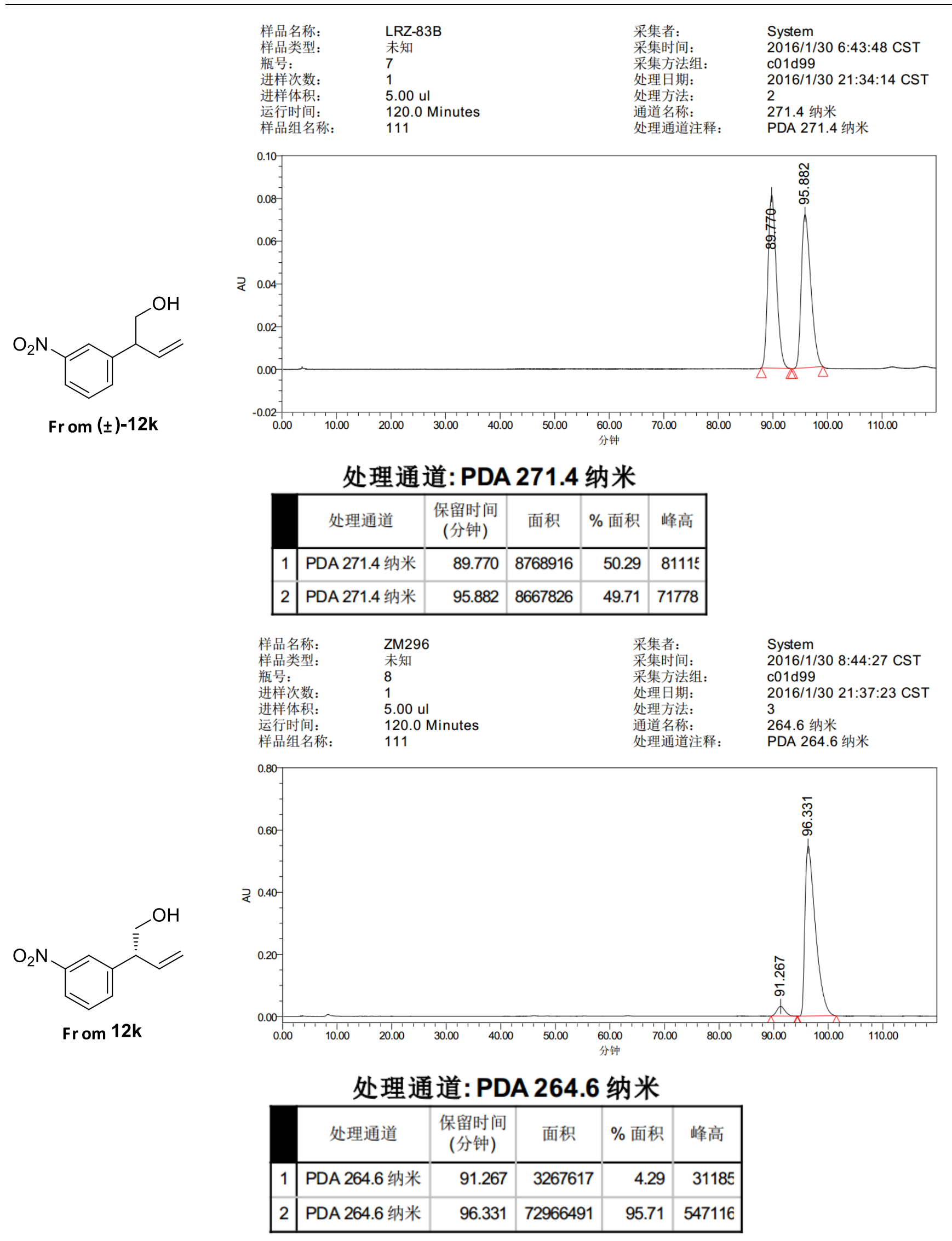


Homoallylic Boronic Esters

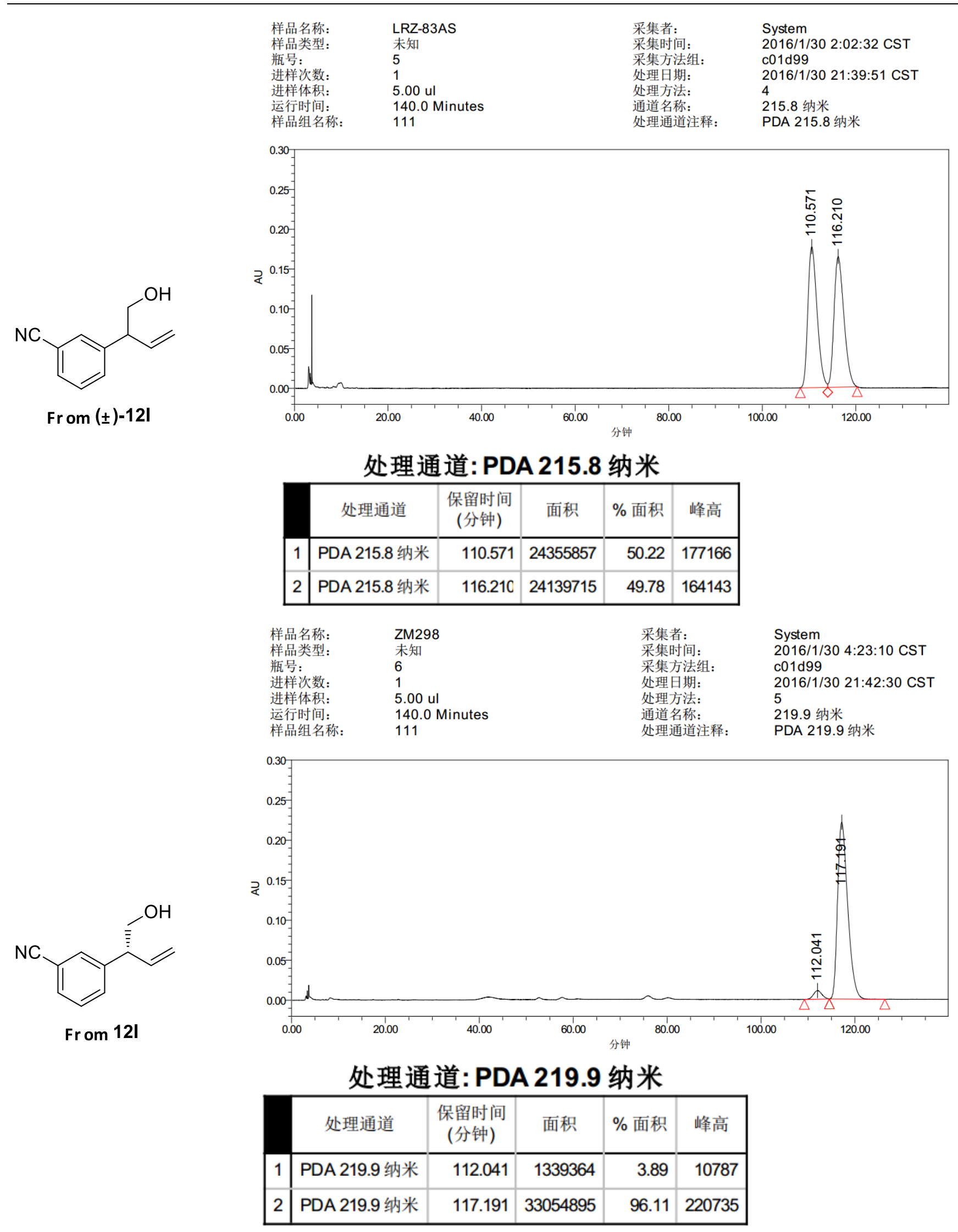


Homoallylic Boronic Esters

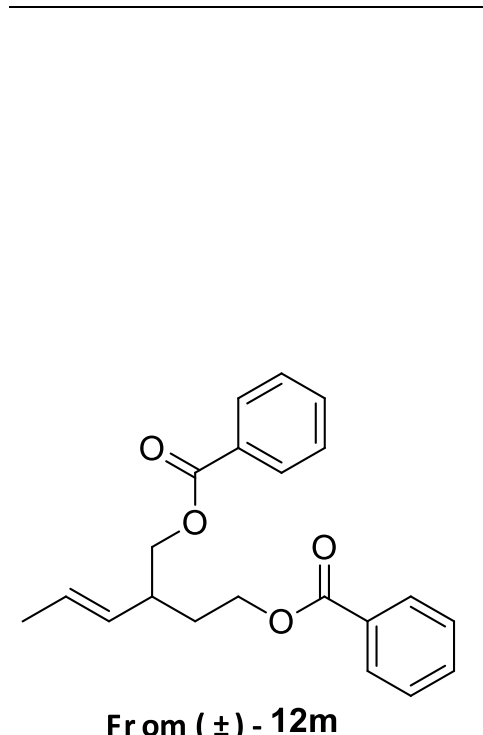

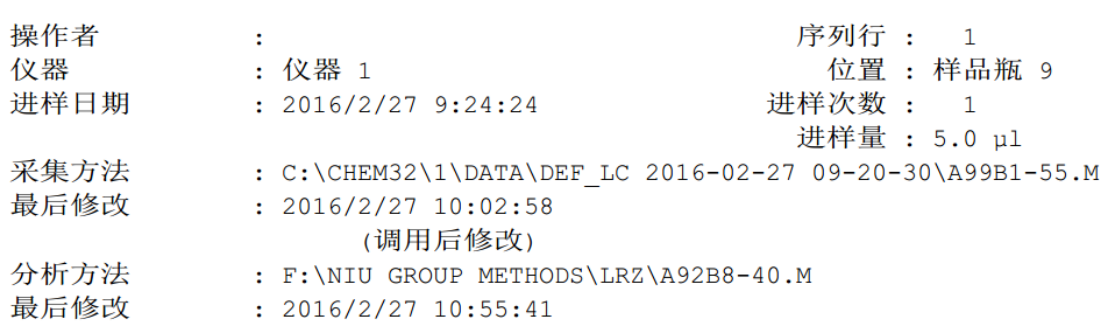

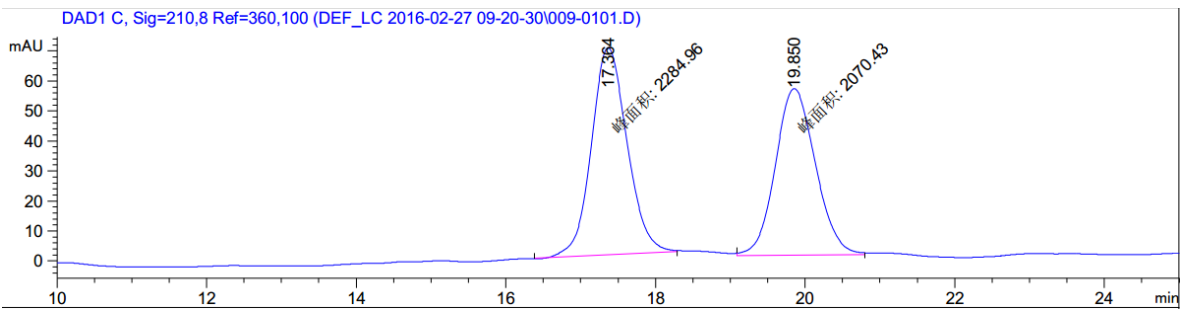

信号 3: DAD1 C, Sig=210,8 $\operatorname{Ref}=360,100$

\begin{tabular}{|c|c|c|c|c|c|c|}
\hline $\begin{array}{r}\text { 峰 } \\
\#\end{array}$ & $\begin{array}{c}\text { 保留时间 } \\
\text { [min] }\end{array}$ & 类型 & $\begin{array}{l}\text { 峰宽 } \\
\text { [min] }\end{array}$ & $\begin{array}{r}\text { 峰面积 } \\
{\left[\mathrm{mAU}^{*} \mathrm{~S}\right]}\end{array}$ & $\begin{array}{r}\text { 峰高 } \\
\text { [mAU] }\end{array}$ & $\begin{array}{c}\text { 峰面积 } \\
\text { 。 }\end{array}$ \\
\hline & ---1 & & - & - & -- & \\
\hline 1 & 17.364 & MM & 0.5519 & 2284.96216 & 69.00832 & 52.4628 \\
\hline 2 & 19.850 & MM & 0.6206 & 2070.43018 & 55.60455 & 47.5372 \\
\hline
\end{tabular}

总量 :

$4355.39233 \quad 124.61286$

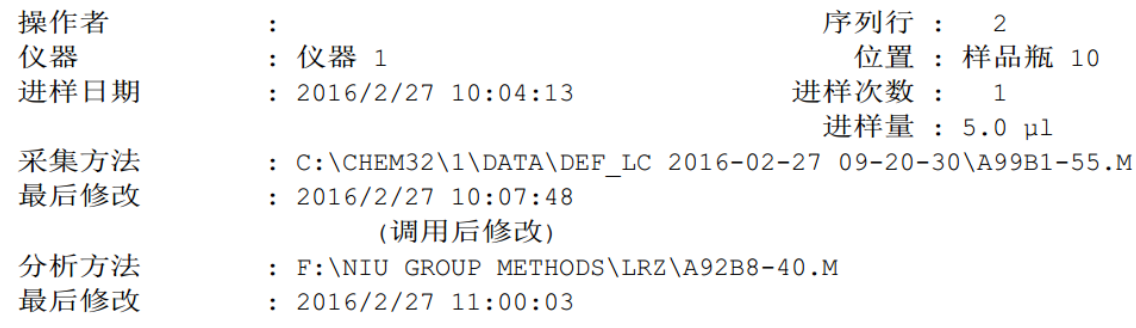<smiles>C/C=C/[C@H](CCOC(=O)c1ccccc1)COC(=O)c1ccccc1</smiles>

From 12m

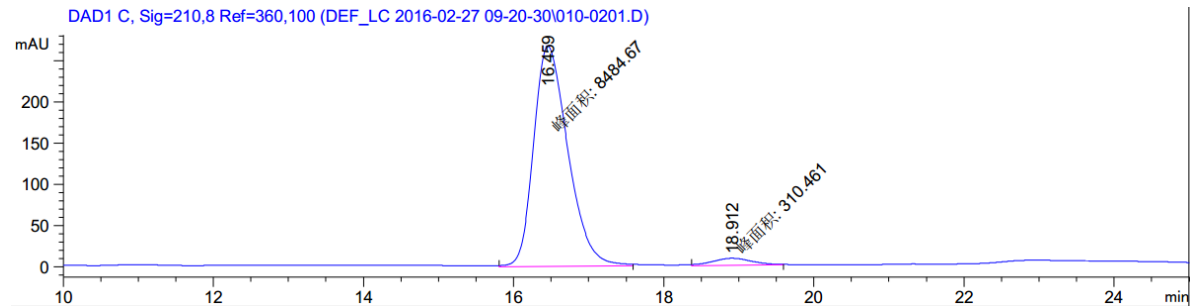

信号 3: DAD1 C, Sig=210,8 $\operatorname{Ref}=360,100$

\begin{tabular}{|c|c|c|c|c|c|c|}
\hline $\begin{array}{r}\text { 峰 } \\
\text { \# }\end{array}$ & $\begin{array}{c}\text { 保留时间 } \\
\text { [min] }\end{array}$ & 类型 & $\begin{array}{l}\text { 峰宽 } \\
\text { [min] }\end{array}$ & $\begin{array}{l}\text { 峰面积 } \\
{\left[\mathrm{mAU}{ }^{*} \mathrm{~S}\right]}\end{array}$ & $\begin{array}{l}\text { 峰高 } \\
\text { [mAU] }\end{array}$ & $\begin{array}{c}\text { 峰面积 } \\
\text { 。 }\end{array}$ \\
\hline-- & | - - - - - & & --- & ---- & ---- & --1 \\
\hline 1 & 16.459 & MM & 284 & 8484.67480 & 267.62097 & 01 \\
\hline 0 & 18.912 & MM & 106 & 310.46069 & 8.47369 & 99 \\
\hline
\end{tabular}


Homoallylic Boronic Esters

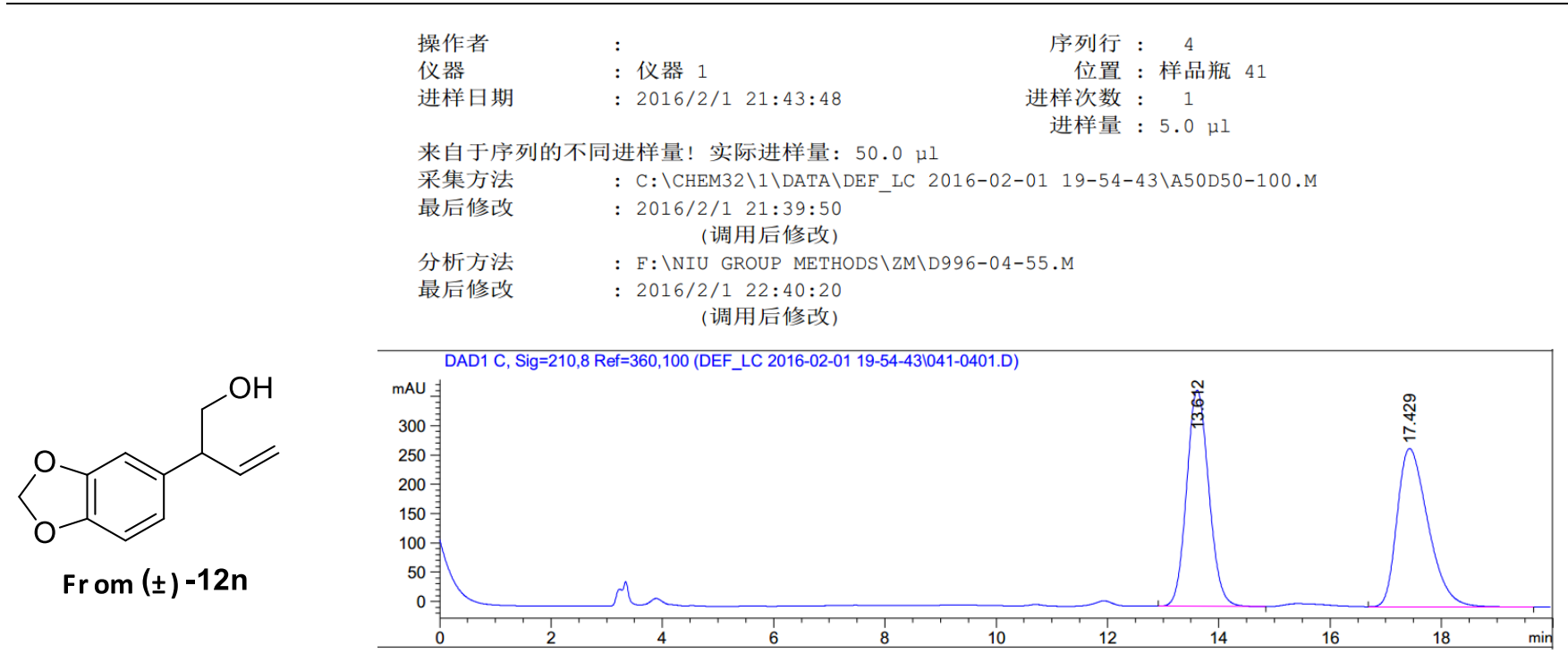

信号 3: DAD1 C, Sig=210,8 $\operatorname{Ref}=360,100$

\begin{tabular}{|c|c|c|c|c|c|c|}
\hline $\begin{array}{r}\text { 峰 } \\
\text { \# }\end{array}$ & $\begin{array}{c}\text { 保留时间 } \\
\text { [min] }\end{array}$ & 类型 & $\begin{array}{l}\text { 峰宽 } \\
\text { [min] }\end{array}$ & $\begin{array}{c}\text { 峰面积 } \\
{\left[m A{ }^{*} s\right]}\end{array}$ & $\begin{array}{l}\text { 峰高 } \\
\text { [mAU] }\end{array}$ & $\begin{array}{c}\text { 峰面积 } \\
\text { 。 }\end{array}$ \\
\hline & & & & . & --- & \\
\hline & & & & 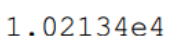 & 274 & \\
\hline ? & & & 72 & $1.04480 \mathrm{e} 4$ & 270 & \\
\hline
\end{tabular}

总量： $\quad 2.06613 e 4 \quad 638.87607$

$\begin{array}{llc}\text { 操作者 } & : & \text { 序列行 : } 4 \\ \text { 仪器 } & \text { 仪器 } 1 & \text { 位置 }: \text { 样品瓶 } 42 \\ \text { 进样日期 } & : \text { : 2016/2/1 18:26:06 } & \text { 进样次数 }: 11\end{array}$

来自于序列的不同进样量! 实际进样量： $3.0 \mu 1$

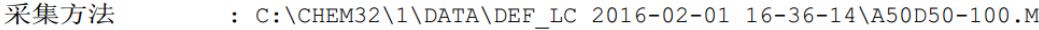

最后修改 : 2016/2/1 18:02:47

分析方法 $:$ F $: \backslash N I U$ GROUP METHODS $\backslash Z M \backslash D 996-04-55 . M$

最后修改 : 2016/2/1 22:40:20

(调用后修改)<smiles>C=C[C@@H](CO)c1ccc2c(c1)OCO2</smiles>

From 12n

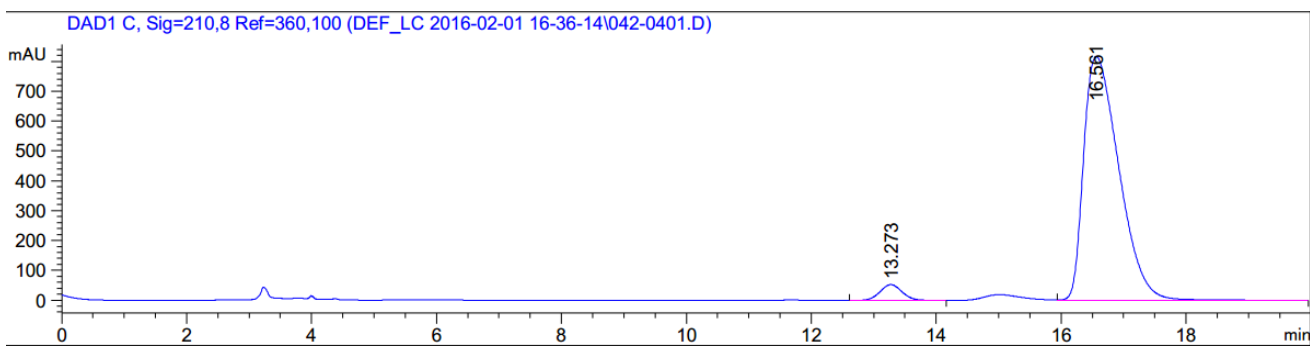

信号 3: DAD1 C, Sig=210,8 Ref $=360,100$

\begin{tabular}{|c|c|c|c|c|c|c|}
\hline $\begin{array}{r}\text { 峰 } \\
\text { \# }\end{array}$ & $\begin{array}{c}\text { 保留时间 } \\
\text { [min] }\end{array}$ & 类型 & $\begin{array}{l}\text { 峰宽 } \\
\text { [min] }\end{array}$ & $\begin{array}{l}\text { 峰面积 } \\
{\left[\mathrm{mAU}^{*} \mathrm{~s}\right]}\end{array}$ & $\begin{array}{r}\text { 峰高 } \\
\text { [mAU] }\end{array}$ & $\begin{array}{c}\text { 峰面积 } \\
\text { 。 }\end{array}$ \\
\hline &.- & & -1 & - - - - - - & --- & --- \\
\hline 1 & 3.2 & & 4 & 1307.0 & 569 & 16 \\
\hline 2 & 61 & $\mathrm{BA}$ & 158 & $3.21080 \mathrm{e} 4$ & 7914 & 96 \\
\hline
\end{tabular}

总量： $\quad 3.34150 \mathrm{e} 4 \quad 871.24483$ 
Homoallylic Boronic Esters

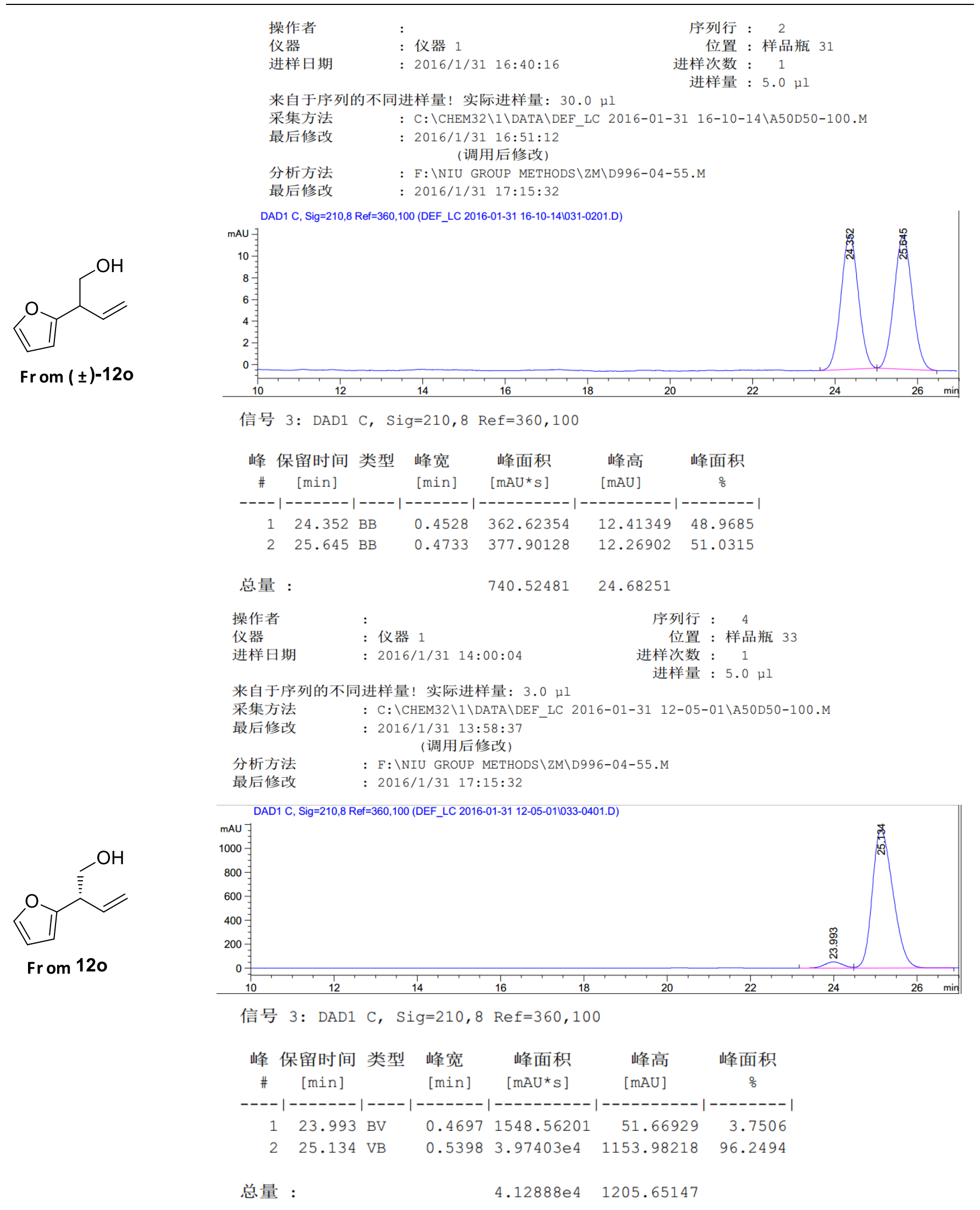


Homoallylic Boronic Esters<smiles>C=CC(CB1OC(C)(C)C(C)(C)O1)c1ccc2c(ccn2C)c1</smiles>

$( \pm)-12 p$

\begin{tabular}{|c|c|c|c|}
\hline 样品名称: & $281-R$ & 采集者: & System \\
\hline 样品类型: & 标准样 & 采集时间: & 2016/1/27 18:59:17 CST \\
\hline 瓶号: & 14 & 采集方法组: & $\mathrm{c} 01 \mathrm{~d} 99$ \\
\hline 进样次数: & 1 & 处理日期: & 2016/1/31 13:38:19 CST \\
\hline 进样体积: & $10.00 \mathrm{ul}$ & 处理方法: & 16 \\
\hline 运行时间: & 10.0 Minutes & 通道名称: & 224.0 纳米 \\
\hline 样品组名称: & LRZ & 处理通道注释: & PDA 224.0 纳米 \\
\hline
\end{tabular}

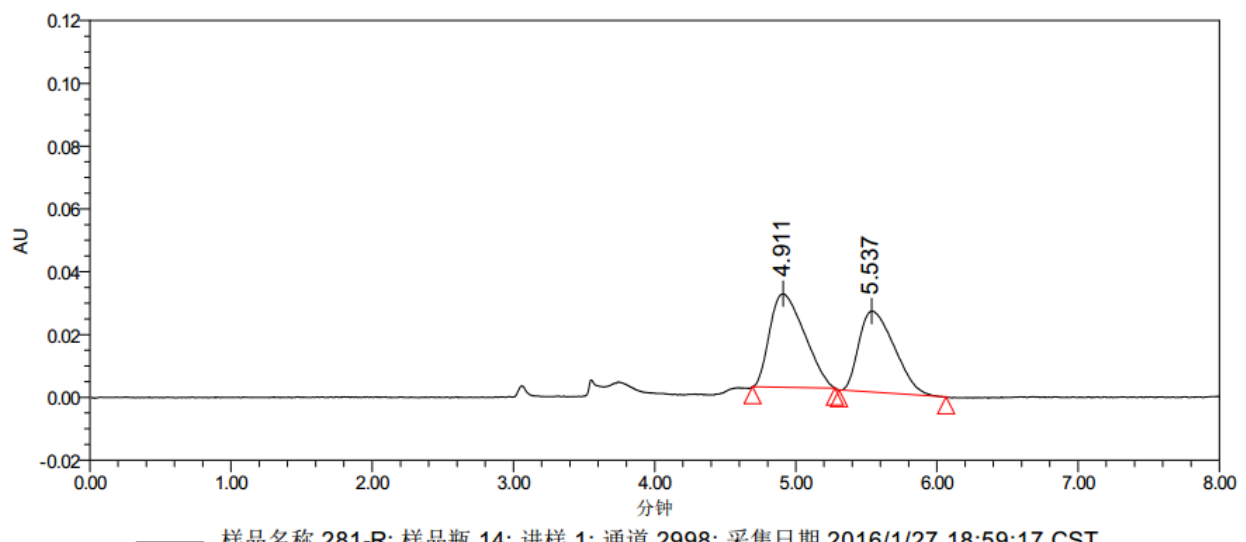

样品名称 281-R; 样品瓶 14; 进样 1; 通道 2998; 采集日期 2016/1/27 18:59:17 CST

处理通道: PDA 224.0 纳米

\begin{tabular}{|r|l|r|r|r|r|}
\hline & 处理通道 & $\begin{array}{c}\text { 保留时间 } \\
\text { (分钟) }\end{array}$ & 面积 & \% 面积 & 峰高 \\
\hline 1 & PDA 224.0 纳米 & 4.911 & 508451 & 53.03 & 29798 \\
\hline 2 & PDA 224.0 纳米 & 5.537 & 450351 & 46.97 & 25775 \\
\hline
\end{tabular}

\begin{tabular}{llll}
\hline \hline & & & \\
样品名称: & ZM-285 & 采集者: & System \\
样品类型: & 标准样 & 采集时间: & $2016 / 1 / 27$ 12:50:31 CST \\
瓶号: & 15 & 采集方法组: & c01d99 \\
进样次数: & 1 & 处理日期: & $2016 / 2 / 118: 11: 37$ CST \\
进样体积: & $5.00 \mathrm{ul}$ & 处理方法: & 22 \\
运行时间: & 10.0 Minutes & 通道名称: & 223.2 纳米 \\
样品组名称: & LRZ & 处理通道注释: & PDA 223.2 纳米
\end{tabular}<smiles>C=C[C@@H](CB1OC(C)(C)C(C)(C)O1)c1ccc2c(ccn2C(C)(C)C)c1</smiles>

$12 p$

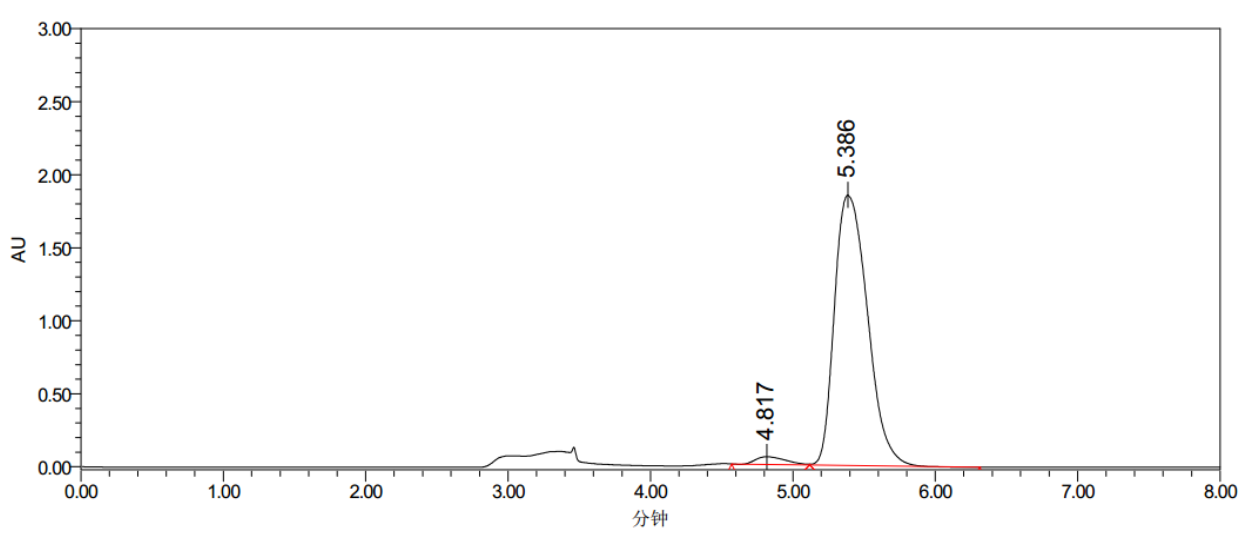

处理通道: PDA 223.2 纳米

\begin{tabular}{|r|c|r|r|r|r|}
\hline & 处理通道 & $\begin{array}{r}\text { 保留时间 } \\
\text { (分钟) }\end{array}$ & 面积 & \% 面积 & 峰高 \\
\hline 1 & PDA 223.2 纳米 & 4.817 & 771732 & 2.52 & 52476 \\
\hline 2 & PDA 223.2 纳米 & 5.386 & 29835802 & 97.48 & 1853008 \\
\hline
\end{tabular}




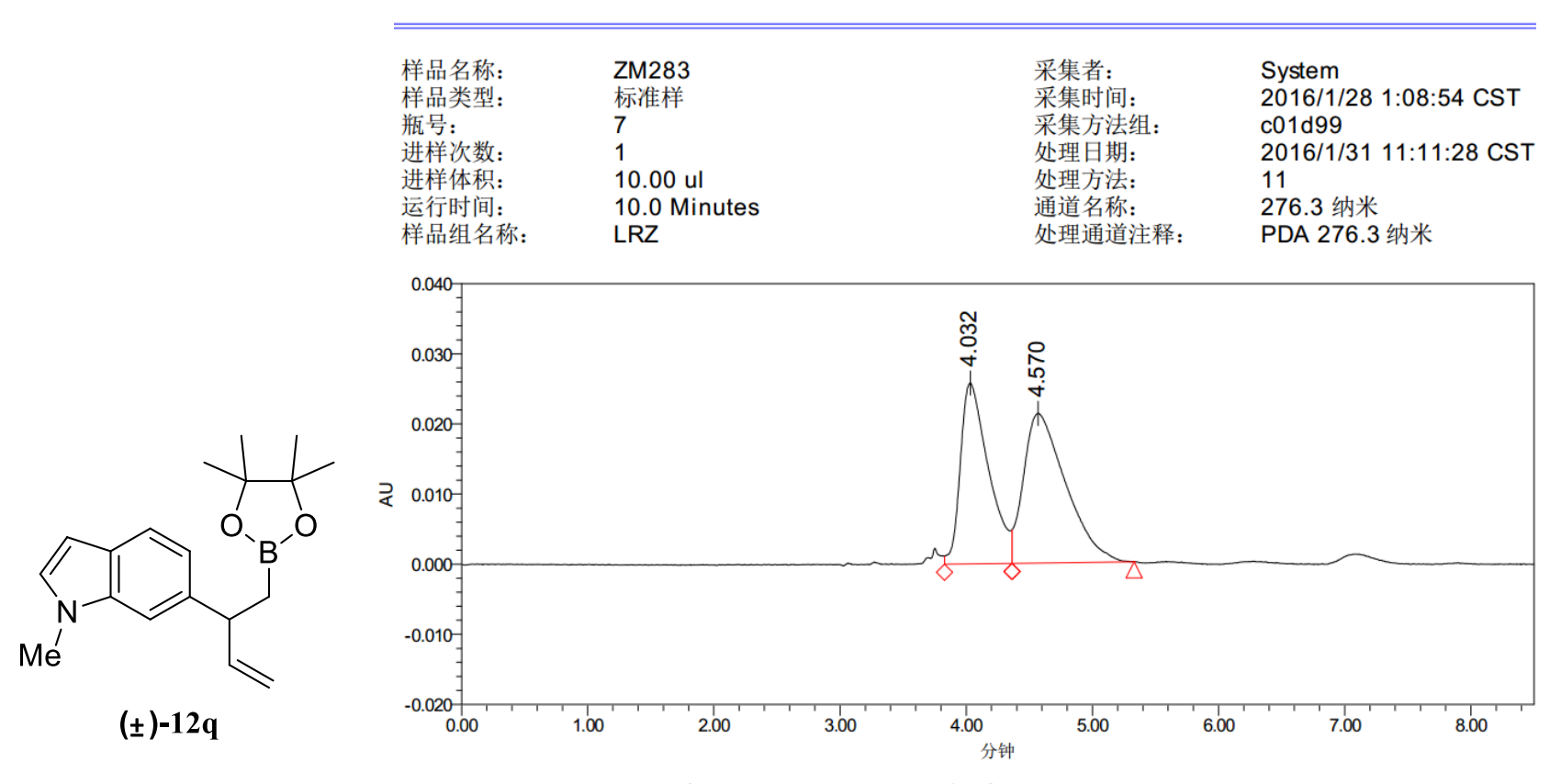

处理通道: PDA 276.3 纳米

\begin{tabular}{|r|c|r|r|r|r|}
\hline & 处理通道 & $\begin{array}{c}\text { 保留时间 } \\
\text { (分钟) }\end{array}$ & 面积 & \% 面积 & 峰高 \\
\hline 1 & PDA 276.3 纳米 & 4.032 & 400284 & 44.67 & 25771 \\
\hline 2 & PDA 276.3 纳米 & 4.570 & 495839 & 55.33 & 21331 \\
\hline
\end{tabular}

\begin{tabular}{llll}
\hline \hline & & & \\
样品名称: & ZM-287 & 采集者: & System \\
样品类型: & 未知 & 采集时间: & $2016 / 1 / 274: 24: 21$ CST \\
瓶号: & 8 & 采集方法组: & c01d99 \\
进样次数: & 1 & 处理日期: & $2016 / 1 / 3111: 25: 01$ CST \\
进样体积: & $5.00 \mathrm{ul}$ & 处理方法: & 12 \\
运行时间: & 10.0 Minutes & 通道名称: & 214.4 纳米 \\
样品组名称: & $\mathrm{Ir}$ & 处理通道注释: & PDA 214.4 纳米
\end{tabular}<smiles>C=C[C@@H](CB1OC(C)(C)C(C)(C)O1)c1ccc2ccn(C)c2c1</smiles>

$12 q$

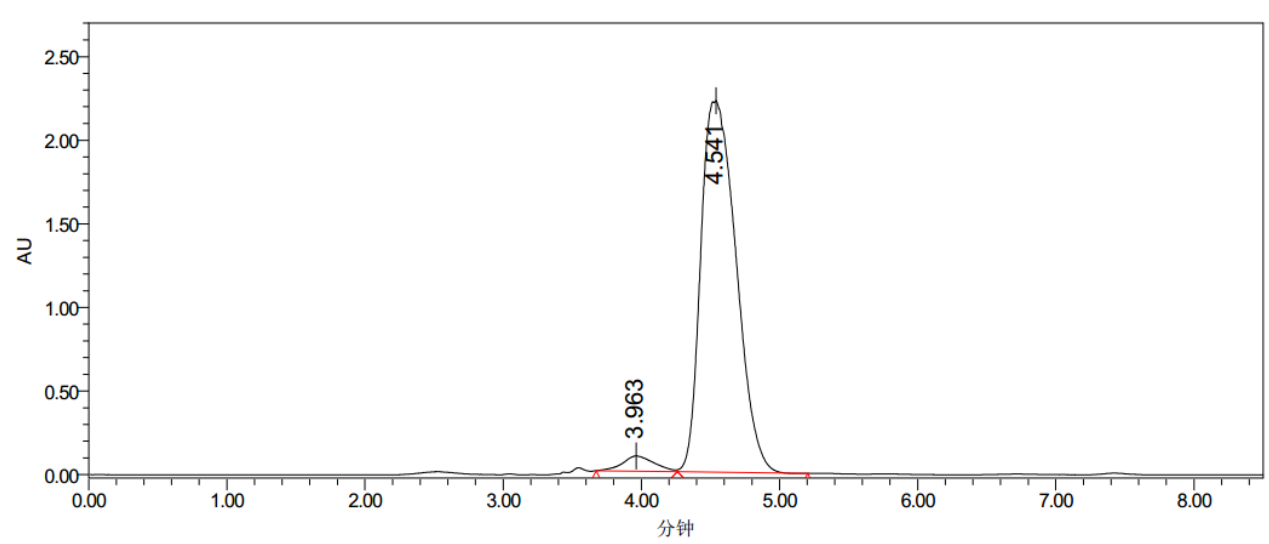

处理通道: PDA 214.4 纳米

\begin{tabular}{|r|c|r|r|r|r|}
\hline & 处理通道 & $\begin{array}{c}\text { 保留时间 } \\
\text { (分钟) }\end{array}$ & 面积 & \% 面积 & 峰高 \\
\hline 1 & PDA 214.4 纳米 & 3.963 & 1469190 & 3.59 & 91025 \\
\hline 2 & PDA 214.4 纳米 & 4.541 & 39403130 & 96.41 & 2222315 \\
\hline
\end{tabular}


Homoallylic Boronic Esters

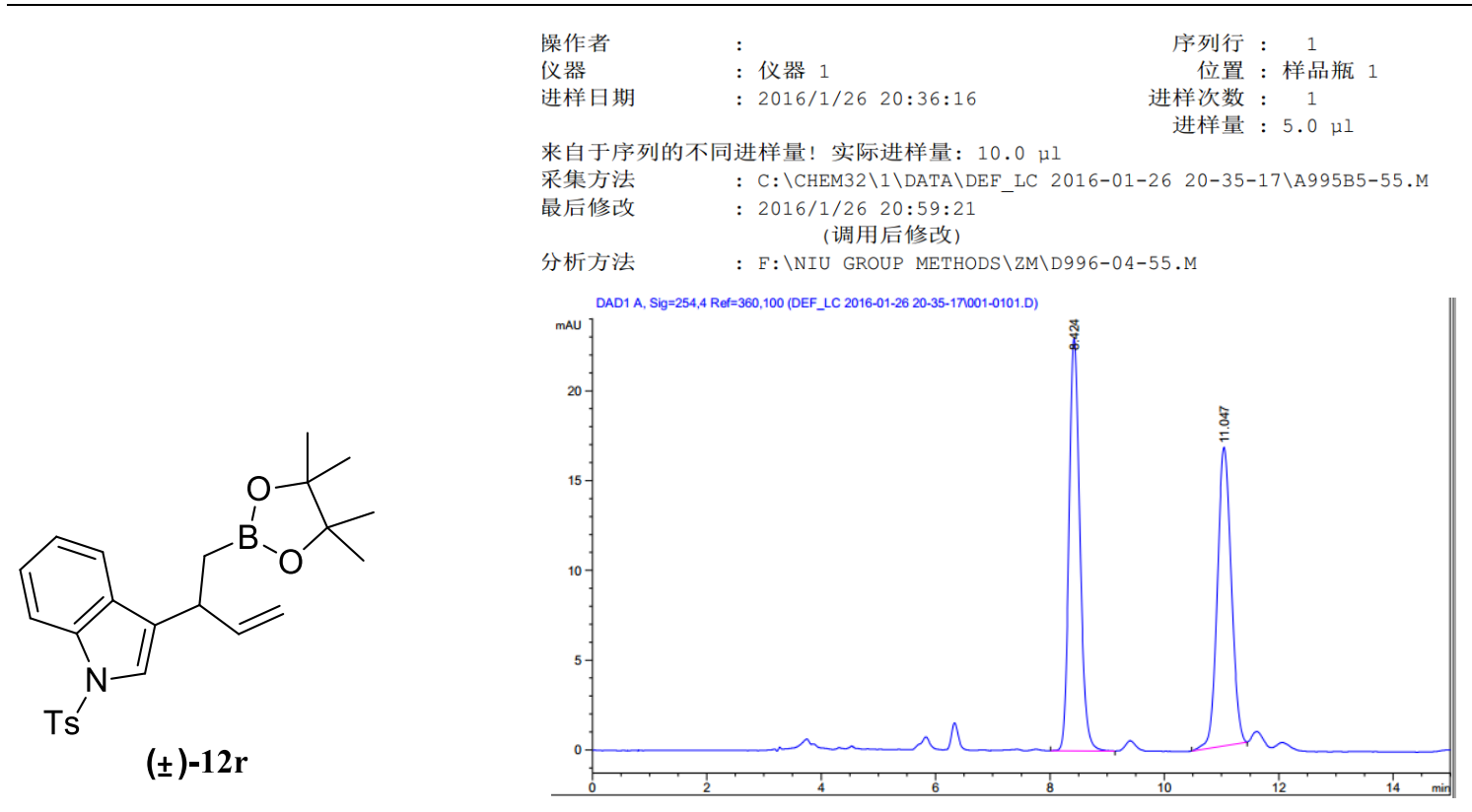

信号 1: DAD1 A, Sig=254,4 Ref=360,100

\begin{tabular}{|c|c|c|c|c|c|c|}
\hline $\begin{array}{r}\text { 峰 } \\
\text { \# }\end{array}$ & $\begin{array}{c}\text { 保留时间 } \\
\text { [min] }\end{array}$ & 类型 & $\begin{array}{l}\text { 峰宽 } \\
\text { [min] }\end{array}$ & $\begin{array}{r}\text { 峰面积 } \\
{\left[\mathrm{mAU}{ }^{*} \mathrm{~S}\right]}\end{array}$ & $\begin{array}{r}\text { 峰高 } \\
\text { [mAU] }\end{array}$ & $\begin{array}{c}\text { 峰面积 } \\
\text { 。 }\end{array}$ \\
\hline--- & ----- & & ------- & $----------\mid$ & -------- & -------- \\
\hline 1 & 8.424 & $\mathrm{BB}$ & 0.2009 & 300.95462 & 22.96175 & 51.6540 \\
\hline 2 & 11.047 & $\mathrm{BB}$ & 0.2617 & 281.68054 & 16.61944 & 48.3460 \\
\hline 总量 & : & & & 582.63516 & 39.58119 & \\
\hline
\end{tabular}

操作者背序列行： 2

仪器哭 1 仪器 1 样品瓶 2

进样日期年进样次数：1 1

来自于序列的不同进样量！实际进样量：10.0 u

采集方法 : C: \CHEM32\1\DATA \DEF_LC 2016-01-26 20-35-17 \A995B5-55.M

最后修改 : 2016/1/26 21:20:33

分析方法 $\quad: F: \backslash N I U$ GROUP METHODS $\backslash Z M \backslash D 996-04-55 . M$

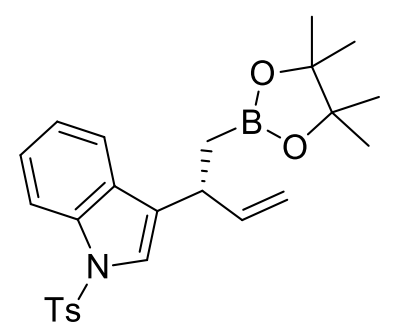

$12 r$

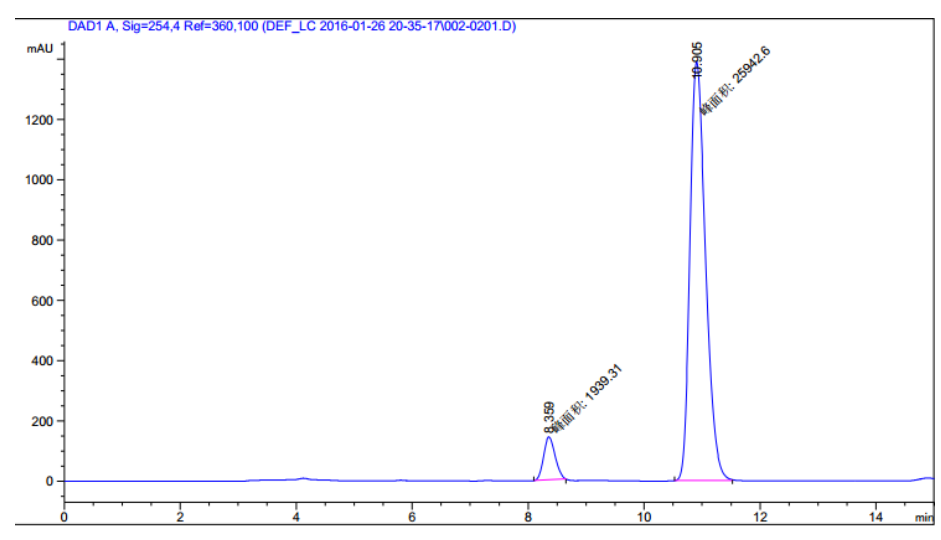

信号 1: DAD1 A, Sig=254,4 Ref=360,100

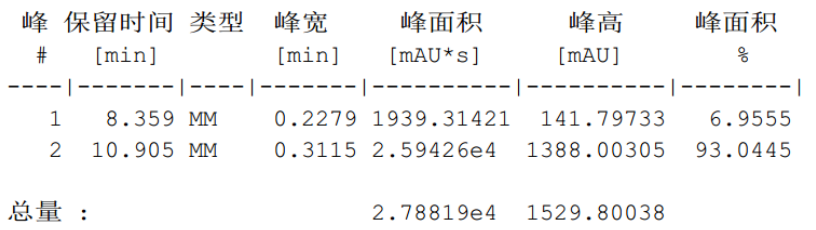


Homoallylic Boronic Esters

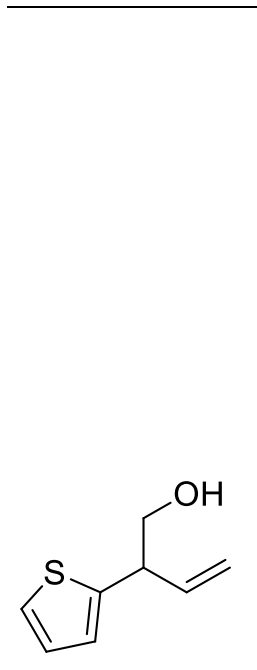

From $( \pm)-12 s$

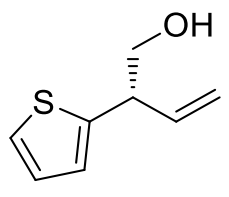

From 12s

$\begin{array}{lll}\text { 操作者 } & : \\ \text { 仪器 } & : \text { 仪器 } 1 \\ \text { 进样日期 } & : \text { : 2016/1/31 20:22:50 }\end{array}$

来自于序列的不同进样量！实际进样量：100.0 $\mu 1$

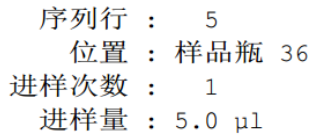

采集方法 ：C：\CHEM32\1\DATA \DEF_LC 2016-01-31 18-18-02\A50D50-100.M

最后修改 : 2016/1/31 20:20:41

(调用后修改)

分析方法 : F : \NIU GROUP METHODS \ZM \D996-04-55.M

最后修改 ：2016/1/31 20:53:56

(调用后修改)

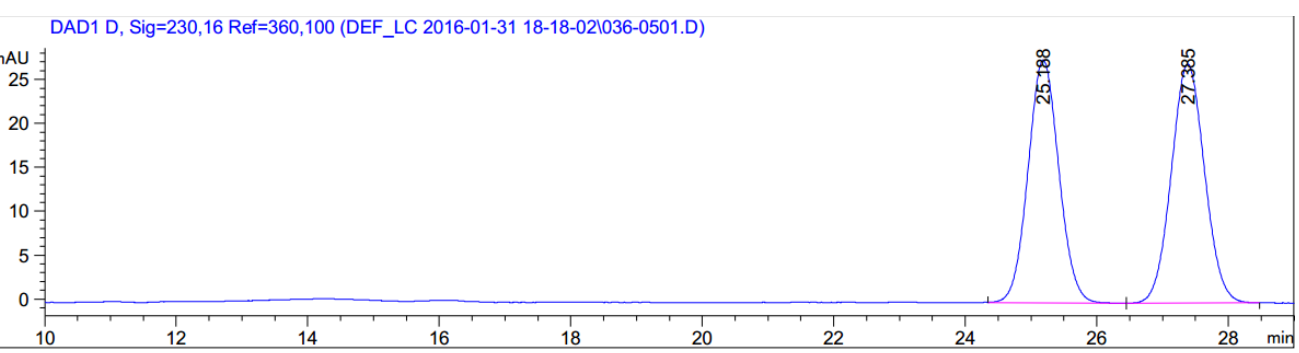

信号 4: DAD1 D, Sig=230,16 Ref=360,100

\begin{tabular}{|c|c|c|c|c|c|c|}
\hline $\begin{array}{r}\text { 峰 } \\
\text { \# }\end{array}$ & $\begin{array}{c}\text { 保留时间 } \\
\text { [min] }\end{array}$ & 类型 & $\begin{array}{l}\text { 峰宽 } \\
\text { [min] }\end{array}$ & $\begin{array}{c}\text { 峰面积 } \\
{\left[m A{ }^{\star}{ }^{*} \mathrm{~s}\right]}\end{array}$ & $\begin{array}{c}\text { 峰高 } \\
\text { [mAU] }\end{array}$ & $\begin{array}{c}\text { 峰面积 } \\
\text { 。 }\end{array}$ \\
\hline-1 & -- & & --- & --- & --- & - \\
\hline 1 & 188 & BB & 083 & 901.11456 & 27.64498 & 48. \\
\hline 2 & 385 & $\mathrm{BB}$ & 468 & 958.32776 & 27.21355 & 51.5 \\
\hline
\end{tabular}

总量 :

$1859.44232 \quad 54.85853$

操作者

仪器

进样日期 : : 仪器 1

: 2016/1/31 18:56:08
序列行 : 2

位置：样品瓶 37 进样次数 : 1

进样量 : $5.0 \mu 1$

来自于序列的不同进样量! 实际进样量： $10.0 \mu \mathrm{I}$
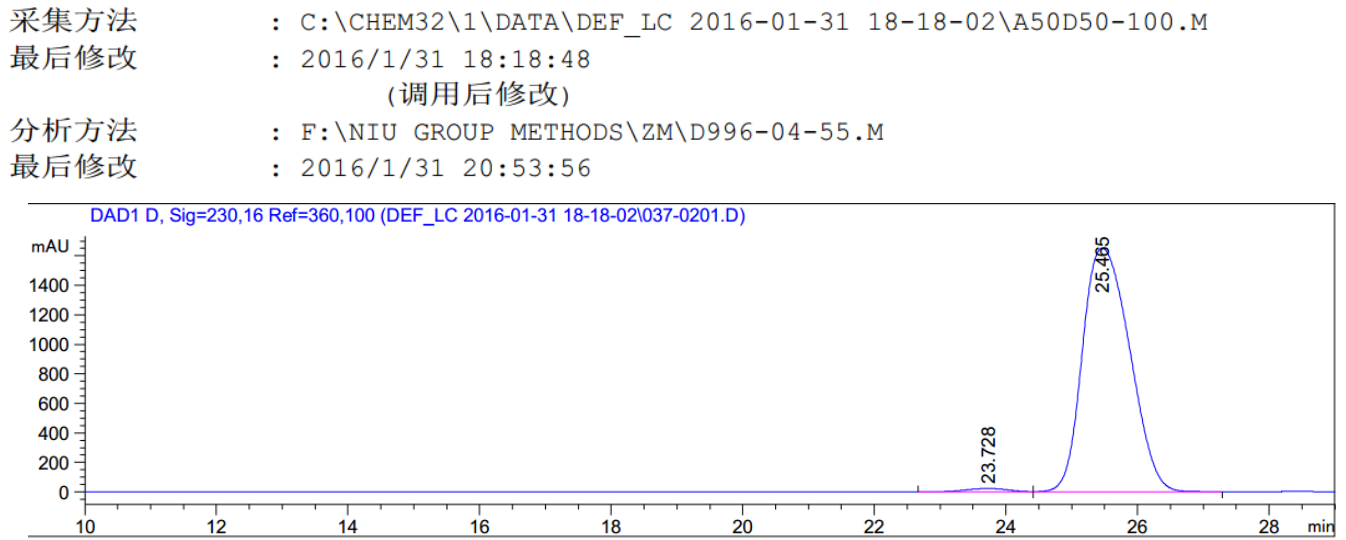

信号 4: DAD1 D, Sig=230,16 Ref $=360,100$

\begin{tabular}{|c|c|c|c|c|c|c|}
\hline $\begin{array}{r}\text { 峰 } \\
\#\end{array}$ & $\begin{array}{c}\text { 保留时间 } \\
\text { [min] }\end{array}$ & 类型 & $\begin{array}{l}\text { 峰宽 } \\
\text { [min] }\end{array}$ & $\begin{array}{l}\text { 峰面积 } \\
{\left[\mathrm{MAU}^{*} \mathrm{~s}\right]}\end{array}$ & $\begin{array}{l}\text { 峰高 } \\
\text { [mAU] }\end{array}$ & $\begin{array}{c}\text { 峰面积 } \\
\text { 。 }\end{array}$ \\
\hline & & & - & - & -- & \\
\hline & 23. & & 83 & 1009.75879 & 2808 & \\
\hline & 25.465 & & 46 & $8.21720 \mathrm{e} 4$ & 1651.21130 & 98 \\
\hline
\end{tabular}

总量：

$8.31817 \mathrm{e} 4 \quad 1673.93938$ 


\section{Homoallylic Boronic Esters}

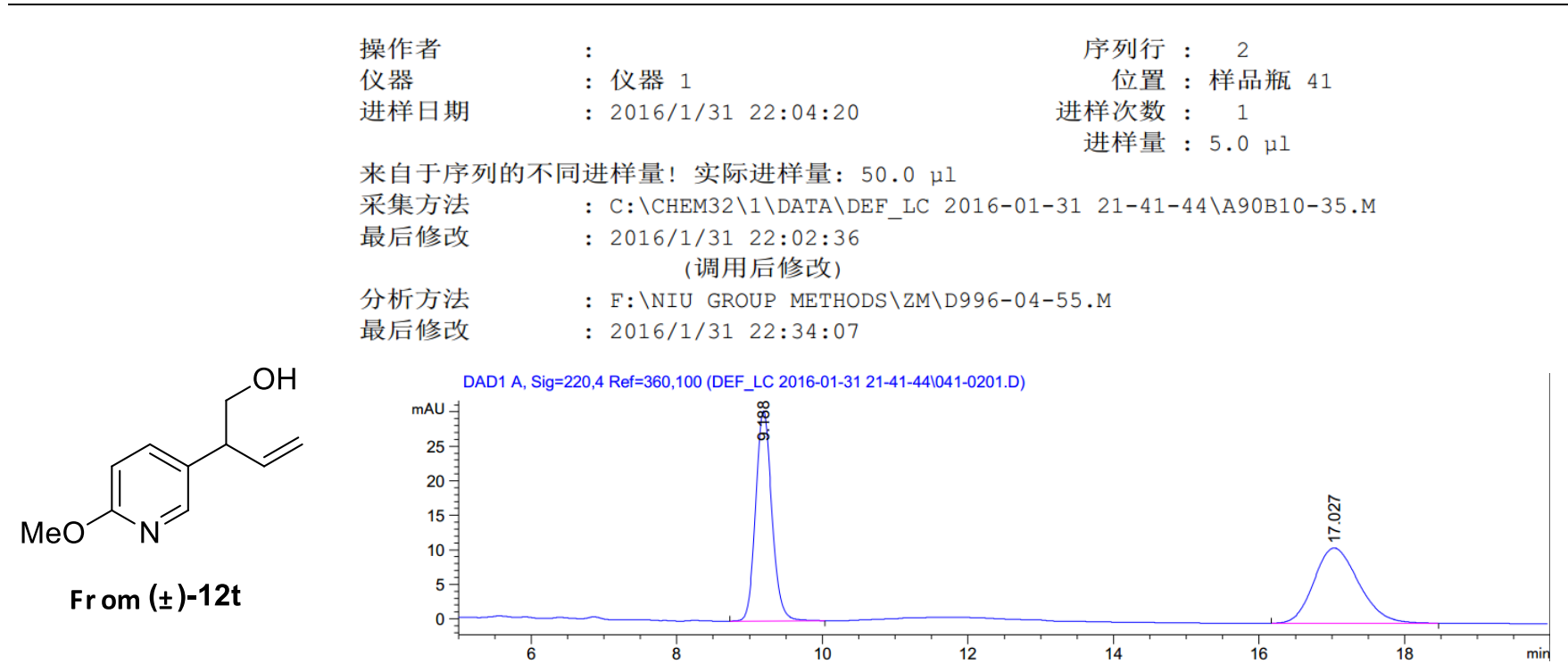

信号 1: DAD1 A, Sig=220,4 Ref=360,100

\begin{tabular}{|c|c|c|c|c|c|c|}
\hline $\begin{array}{r}\text { 峰 } \\
\text { \# }\end{array}$ & $\begin{array}{c}\text { 保留时间 } \\
\text { [min] }\end{array}$ & 类型 & $\begin{array}{l}\text { 峰宽 } \\
\text { [min] }\end{array}$ & $\begin{array}{l}\text { 峰面积 } \\
{\left[\mathrm{mAU}{ }^{*} \mathrm{~S}\right]}\end{array}$ & $\begin{array}{c}\text { 峰高 } \\
\text { [mAU] }\end{array}$ & $\begin{array}{c}\text { 峰面积 } \\
\text { 。 }\end{array}$ \\
\hline- & -- & & $=-$ & ---- & ----- & --- \\
\hline 1 & 9.188 & BB & 385 & 470.96280 & 4506 & 775 \\
\hline 0 & 17.027 & PR & 441 & 465. & 0874 & 49 \\
\hline
\end{tabular}

总量 :

$936.72647 \quad 41.35379$

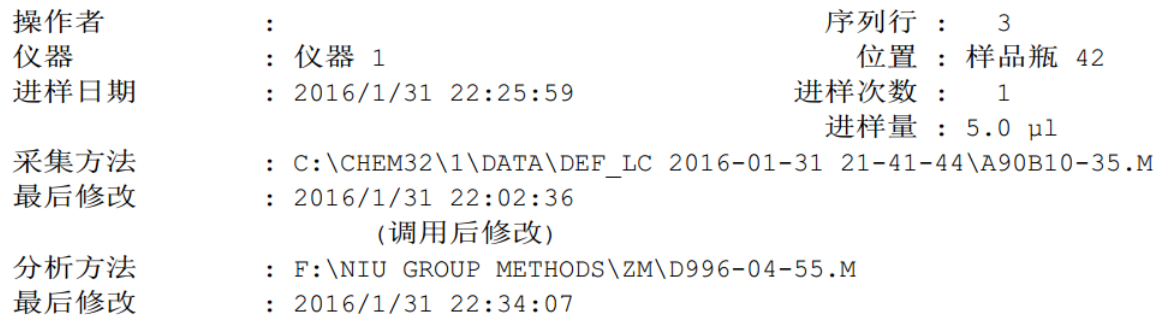<smiles>C=C[C@@H](CO)c1ccc(OC)nc1</smiles>

DAD1 A, Sig=220,4 Ref=360,100 (DEF_LC 2016-01-31 21-41-441042-0301.D)

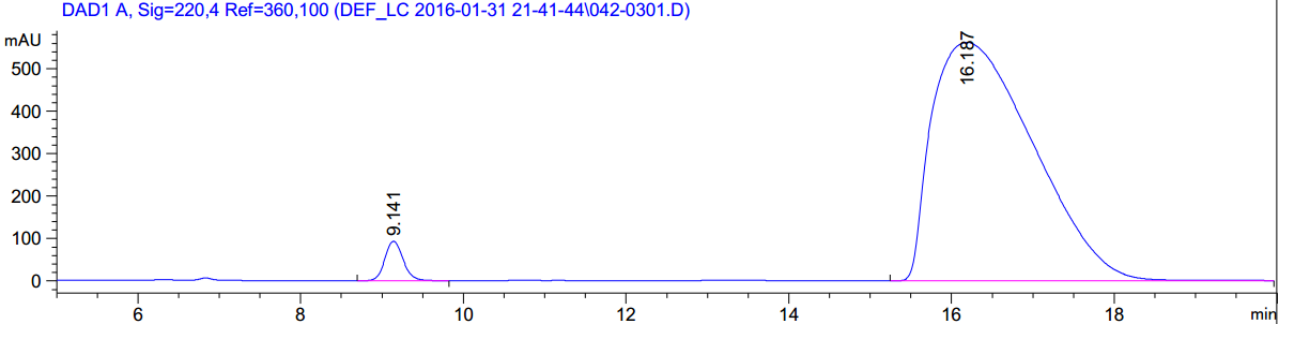

信号 1: DAD1 A, Sig=220,4 Ref=360,100

\begin{tabular}{|c|c|c|c|c|c|c|}
\hline $\begin{array}{r}\text { 峰 } \\
\text { \# }\end{array}$ & $\begin{array}{c}\text { 呆留时间 } \\
\text { [min] }\end{array}$ & 类型 & $\begin{array}{l}\text { 峰宽 } \\
\text { [min] }\end{array}$ & $\begin{array}{l}\text { 峰面积 } \\
{\left[\mathrm{mAU}^{\star} \mathrm{S} \text { ] }\right.}\end{array}$ & $\begin{array}{l}\text { 峰高 } \\
\text { [mAU] }\end{array}$ & $\begin{array}{c}\text { 峰面积 } \\
\text { 。 }\end{array}$ \\
\hline 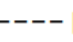 & - & & 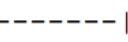 & $\mid---$ & --- & -- \\
\hline 1 & 41 & 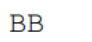 & 402 & 1450.02759 & 3096 & 2.9026 \\
\hline 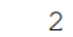 & 16.187 & $\mathrm{BA}$ & .3853 & $4.85053 e 4$ & 561.79187 & 97.0974 \\
\hline
\end{tabular}

总量： 4.99553e4 654.62283 
Homoallylic Boronic Esters

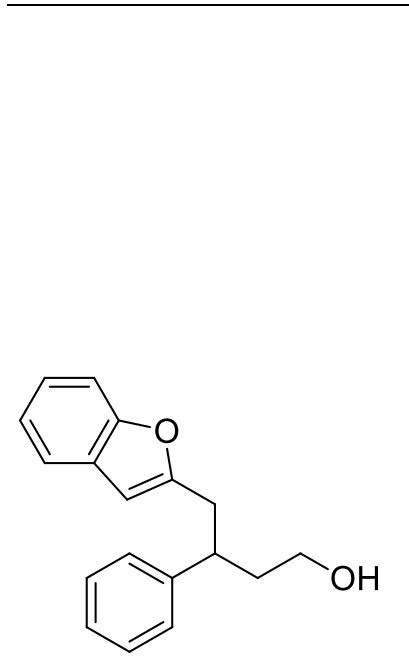

$( \pm)-23$

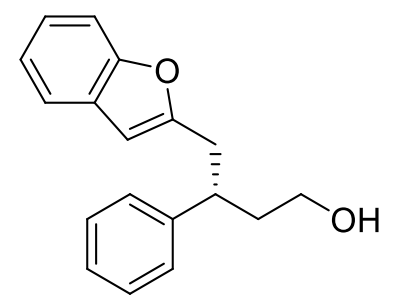

23
: 仪器 1

: 2016/1/28 22:08:34

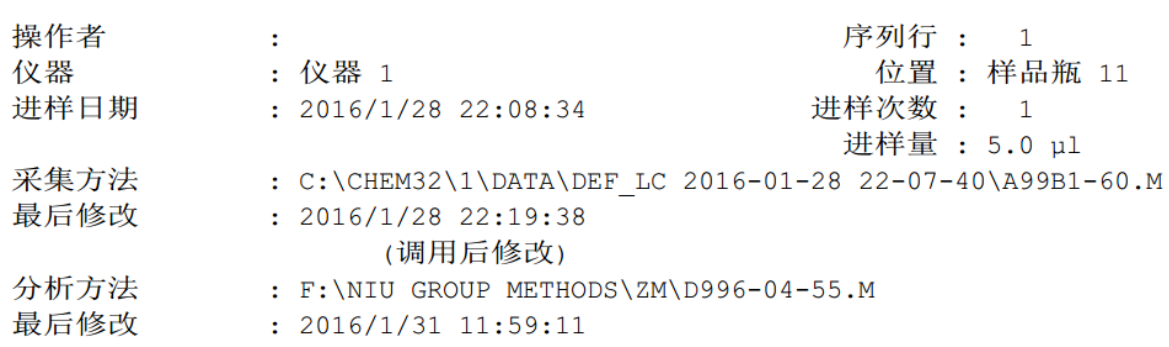

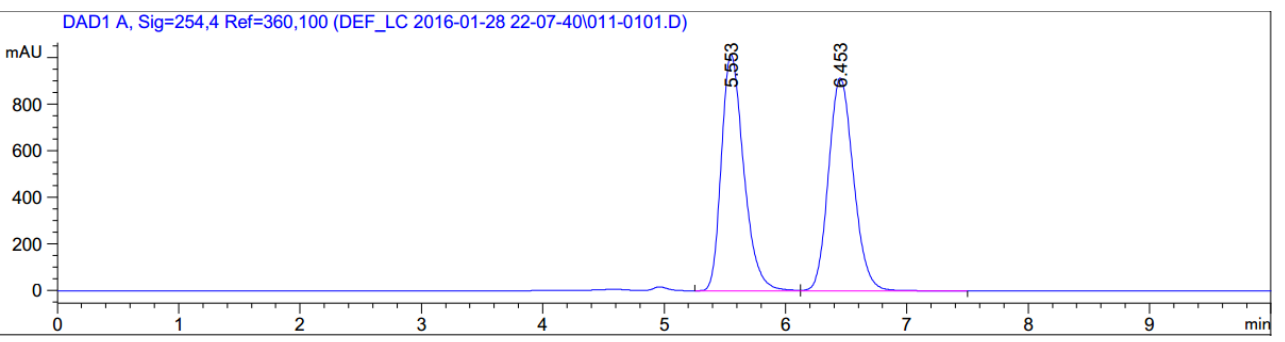

信号 1: DAD1 A, Sig=254,4 Ref=360,100

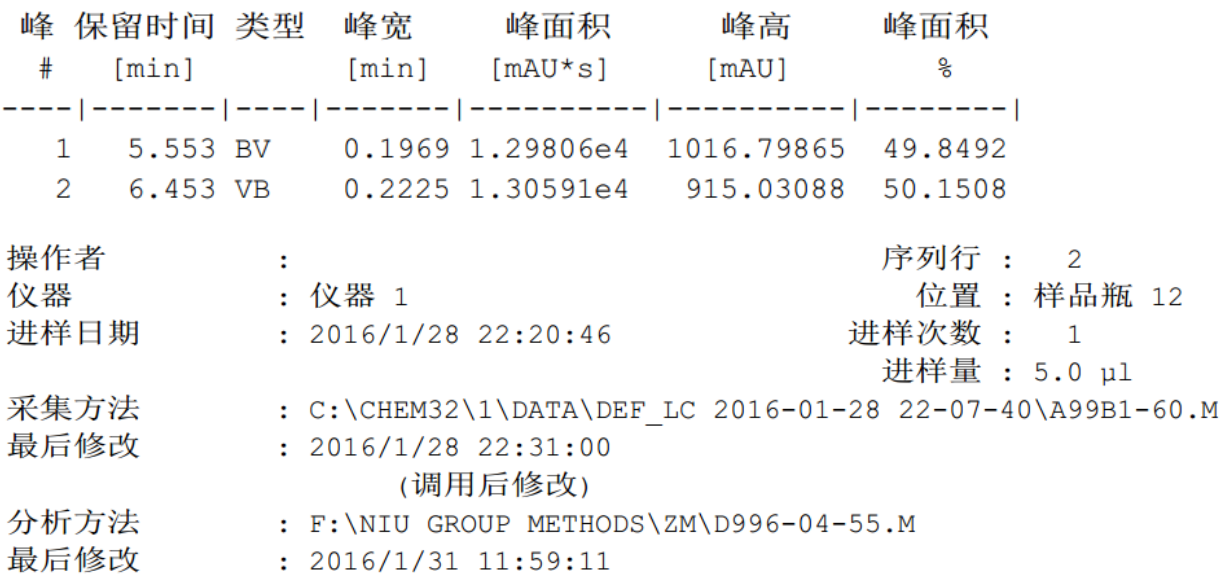

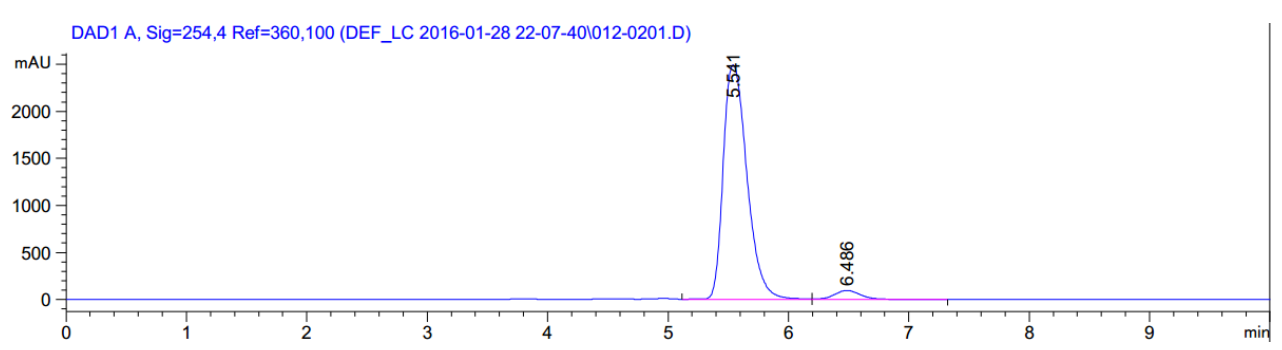

信号 1: DAD1 A, Sig=254, 4 Ref=360, 100

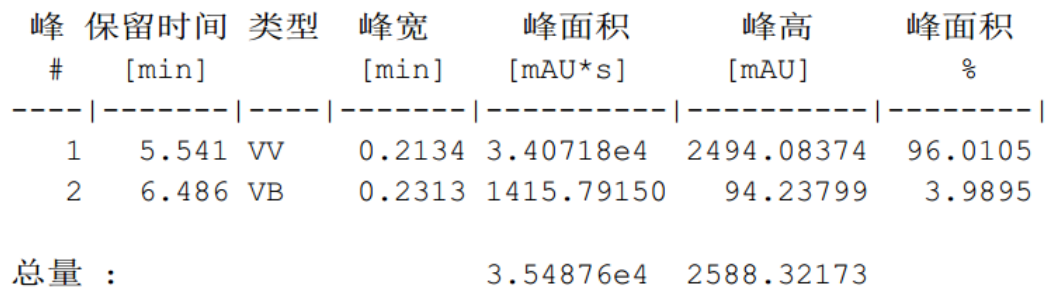


Homoallylic Boronic Esters

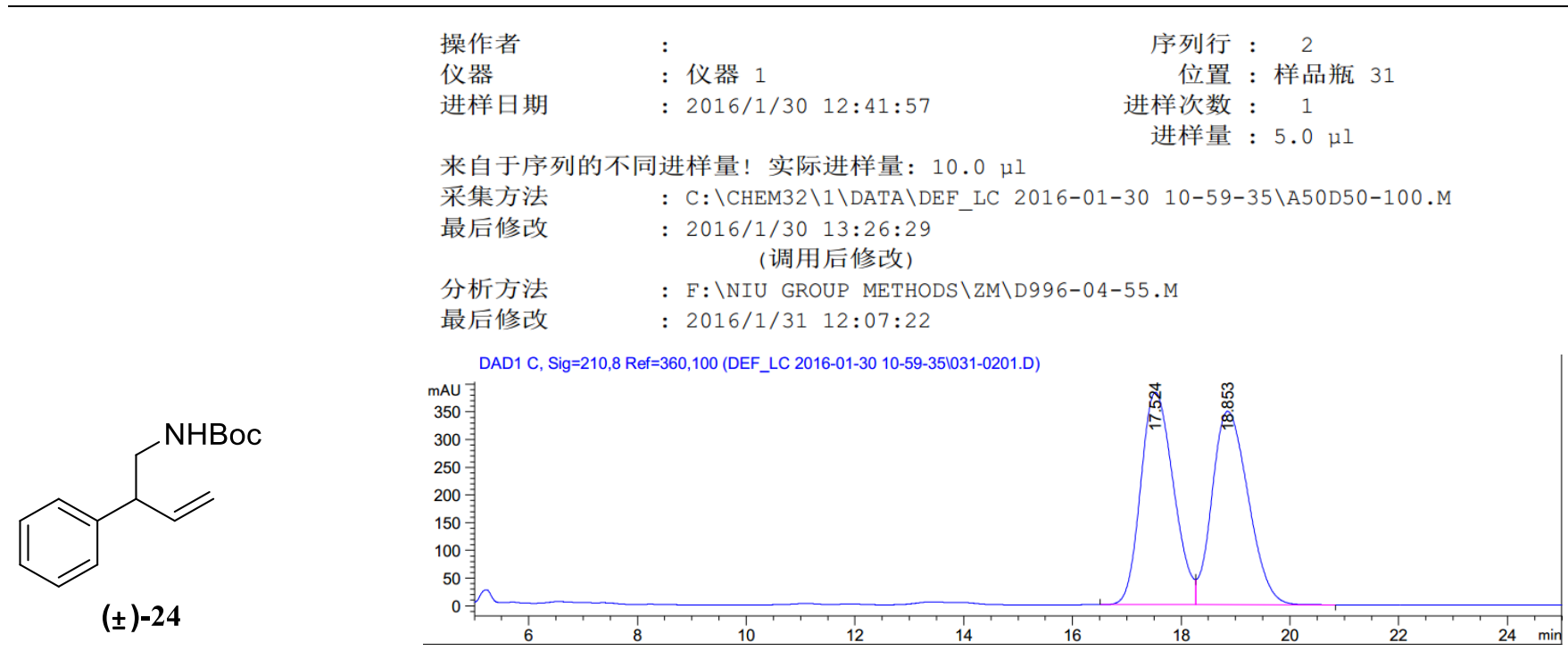

信号 3: DAD1 C, Sig=210,8 Ref $=360,100$

\begin{tabular}{|c|c|c|c|c|c|c|}
\hline $\begin{array}{r}\text { 峰 } \\
\text { \# }\end{array}$ & $\begin{array}{c}\text { 保留时间 } \\
\text { [min] }\end{array}$ & 类型 & $\begin{array}{l}\text { 峰宽 } \\
\text { [min] }\end{array}$ & $\begin{array}{l}\text { 峰面积 } \\
{\left[\mathrm{mAU}{ }^{*} \mathrm{~s}\right]}\end{array}$ & $\begin{array}{l}\text { 峰高 } \\
\text { [mAU] }\end{array}$ & $\begin{array}{c}\text { 峰面积 } \\
\text { 。 }\end{array}$ \\
\hline- & the & & ----- & --------- & -------- & ------- \\
\hline 1 & 17.524 & BV & 0.6581 & $1.60505 \mathrm{e} 4$ & 383.60754 & 49.8282 \\
\hline 2 & 18.853 & VB & 0.7362 & $1.61612 \mathrm{e} 4$ & 349.04108 & 50.1718 \\
\hline 总量 & & & & $3.22117 e 4$ & 732.64862 & \\
\hline
\end{tabular}

\begin{tabular}{|c|c|c|c|}
\hline 操作者 & : & 序列行 & : \\
\hline 仪器 & : & 仪器 1 & ：样品瓶 32 \\
\hline 进样日期 & : & $2016 / 1 / 3013: 35: 28$ & $\begin{array}{l}: \quad 1 \\
: \quad 5.0 \mu l\end{array}$ \\
\hline 采集方法 & e & C: $\backslash$ CHEM32 $\backslash 1 \backslash$ DATA \DEF_LC 2016-01-30 10-59- & $-35 \backslash A 50 D 50-100 . M$ \\
\hline 最后修改 & & $\begin{array}{c}\text { 2016/1/30 13:39:33 } \\
\text { (调用后修改) }\end{array}$ & \\
\hline 方法 & & $\mathrm{F}: \backslash N I U$ GROUP METHODS \LRZ \A90B10-60.M & \\
\hline 晃原 & & $2016 / 2 / 19$ 15:51:10 & \\
\hline
\end{tabular}

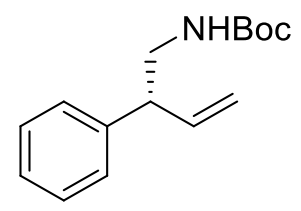

24
DAD1 C, Sig=210,8 Ref=360,100 (DEF_LC 2016-01-30 10-59-35l032-0401.D)

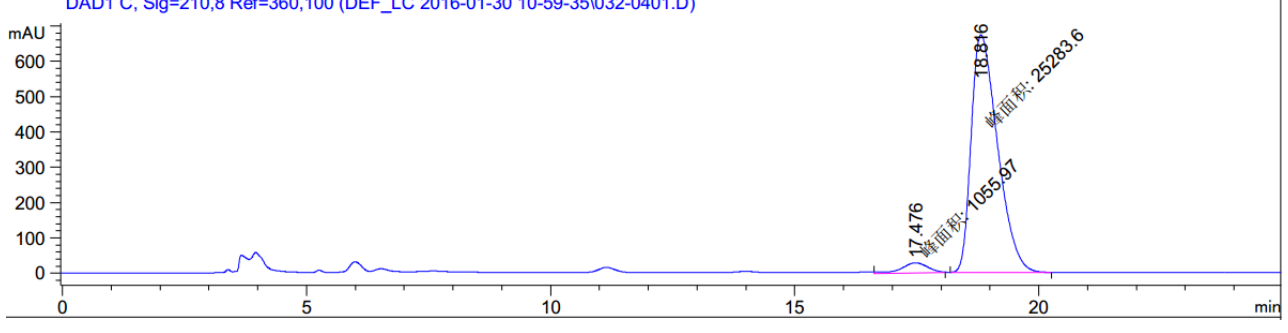

信号 3: DAD1 C, Sig=210,8 Ref=360,100

\begin{tabular}{|c|c|c|c|c|c|c|}
\hline 峰 1 & 保留时间 & 类型 & 峰宽 & 峰面积 & 峰高 & 峰面积 \\
\hline$\#$ & [min] & & [min] & {$\left[\mathrm{mAU}{ }^{*} \mathrm{~s}\right]$} & [mAU] & $\%$ \\
\hline & & & 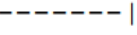 & & -7 & \\
\hline 1 & 17.476 & $\mathrm{MM}$ & 0.6178 & 1055.96899 & 28.48757 & 4 \\
\hline 2 & 18.816 & $\mathrm{D}$ & 263 & $2.52836 e^{4}$ & 012 & 95 \\
\hline
\end{tabular}

总量： $\quad 2.63396 \mathrm{e} 4 \quad 701.29769$ 
Homoallylic Boronic Esters

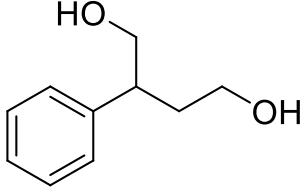

$( \pm)-26$<smiles>OCC[C@@H](CO)c1ccccc1</smiles>

26
SAMPLE INFORMATION

\begin{tabular}{ll}
\hline & \\
Date Acquired: & $4 / 14 / 2016$ 12:31:41 PM CST \\
Sample Name: & DIOL-XXT \\
Vial: & 1:E,2 \\
Injection Volume: & $5.00 \mathrm{ul}$ \\
Acq. Method Set: & $2 \mathrm{~mL} 20$ B4_C4 \\
Acquired By: & System \\
Column: & CHIRALPAK IC-3(4.6*100mm,3um) \\
Co_Solvent: & IPA \\
Column_Temperature: & 35 \\
Co_Solvent\%: & 20 \\
Back_Pressure: & $2000 \mathrm{psi}$ \\
Flow_rate: & $2 \mathrm{~mL} / \mathrm{min}$ \\
Proc. Chnl. Descr.: & PDA 210.0 nm (210-400)nm \\
PDA_Start_Wavelength: & $200 \mathrm{~nm}$ \\
PDA_Stop_Wavelength: & $400 \mathrm{~nm}$
\end{tabular}

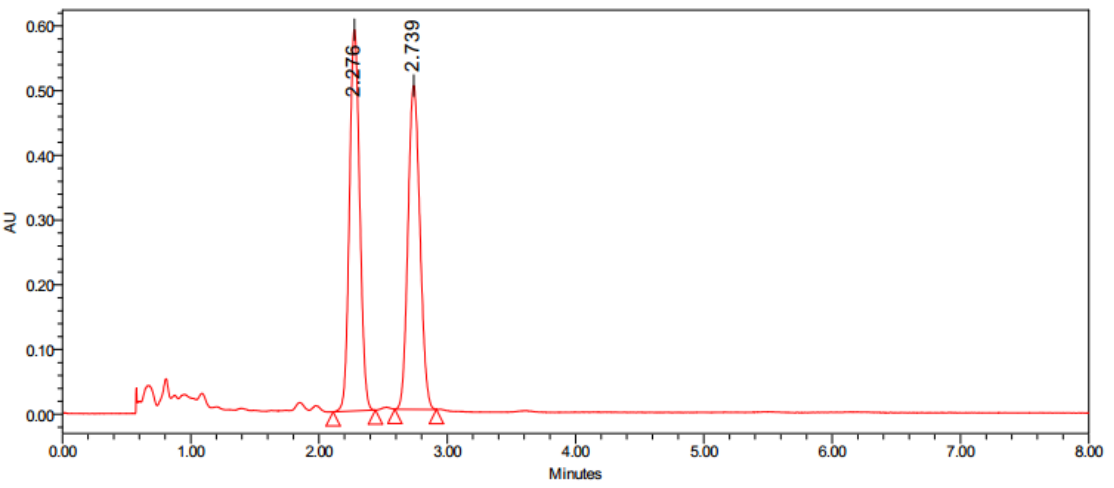

Peak Results
\begin{tabular}{|c|c|c|c|c|}
\hline & RT & Height & Area & \% Area \\
\hline 1 & 2.28 & 589674 & 3272523 & 50.31 \\
\hline 2 & 2.74 & 500495 & 3232632 & 49.69 \\
\hline
\end{tabular}

SAMPLE INFORMATION

Date Acquired: $\quad$ 4/14/2016 1:06:27 PM CST

Sample Name: $\quad$ DIOL

$\begin{array}{ll}\text { Vial: } & 1: E, 3 \\ \text { Injection Volume: } & 5.00 \mathrm{ul}\end{array}$

$\begin{array}{ll}\text { Injection Volume: } & 5.00 \mathrm{ul} \\ \text { Acq. Method Set: } & 2 \mathrm{~mL}-20 \mathrm{~B} 4 \text { CC4 }\end{array}$

Acquired By: $\quad$ System

CHIRALPAK IC-3(4.6*100mm,3um)

Co_Solvent: IPA

Column_Temperature: $\quad 35$

Co_Solvent\%: $\quad 20$

Back_Pressure: 2000psi

Flow_rate: $\quad 2 \mathrm{~mL} / \mathrm{min}$

Proc. Chnl. Descr.: $\quad$ PDA $210.0 \mathrm{~nm}(210-400) \mathrm{nm}$

PDA_Start_Wavelength: $\quad 200 \mathrm{~nm}$

PDA_Stop_Wavelength: $\quad 400 \mathrm{~nm}$

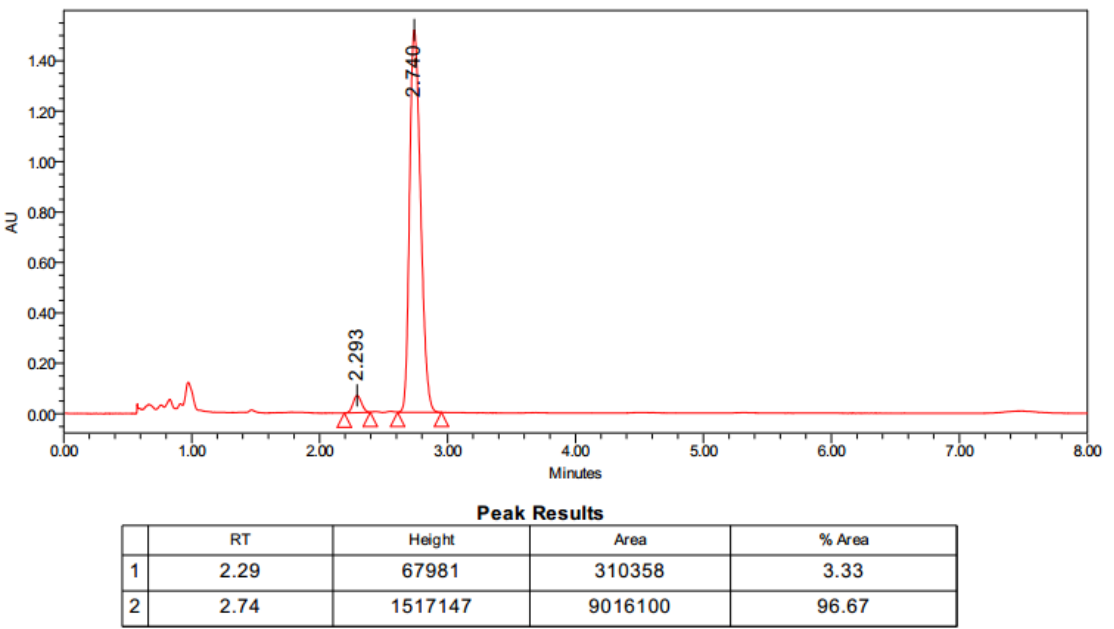


in $\mathrm{CDCl}_{3}, 400 \mathrm{MHz}$
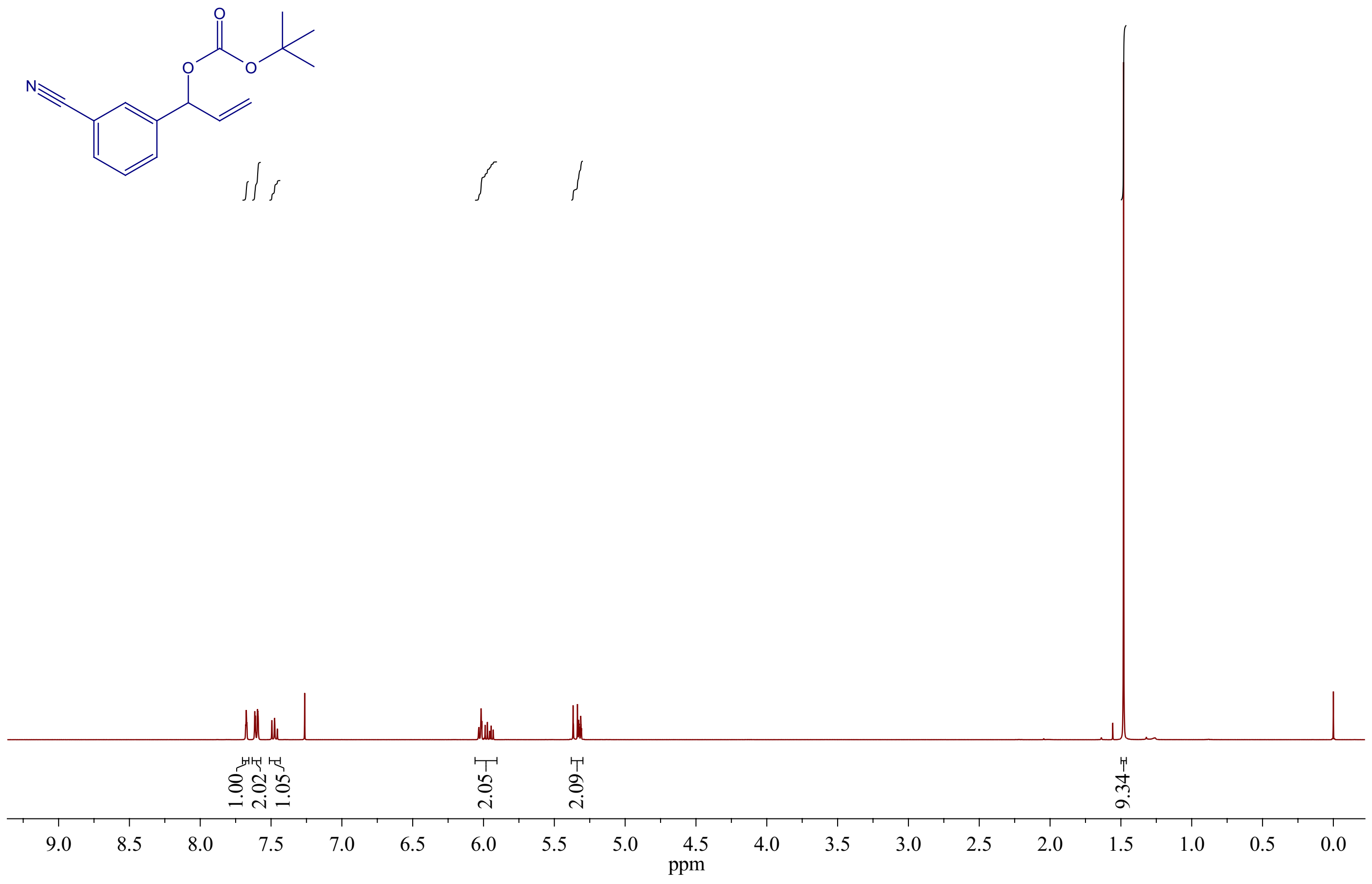


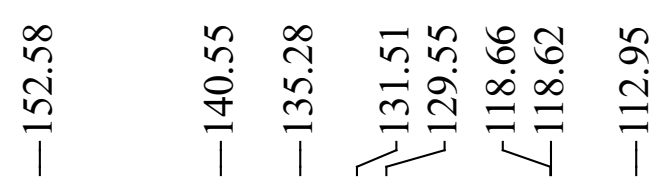

in $\mathrm{CDCl}_{3}, 101 \mathrm{MHz}$
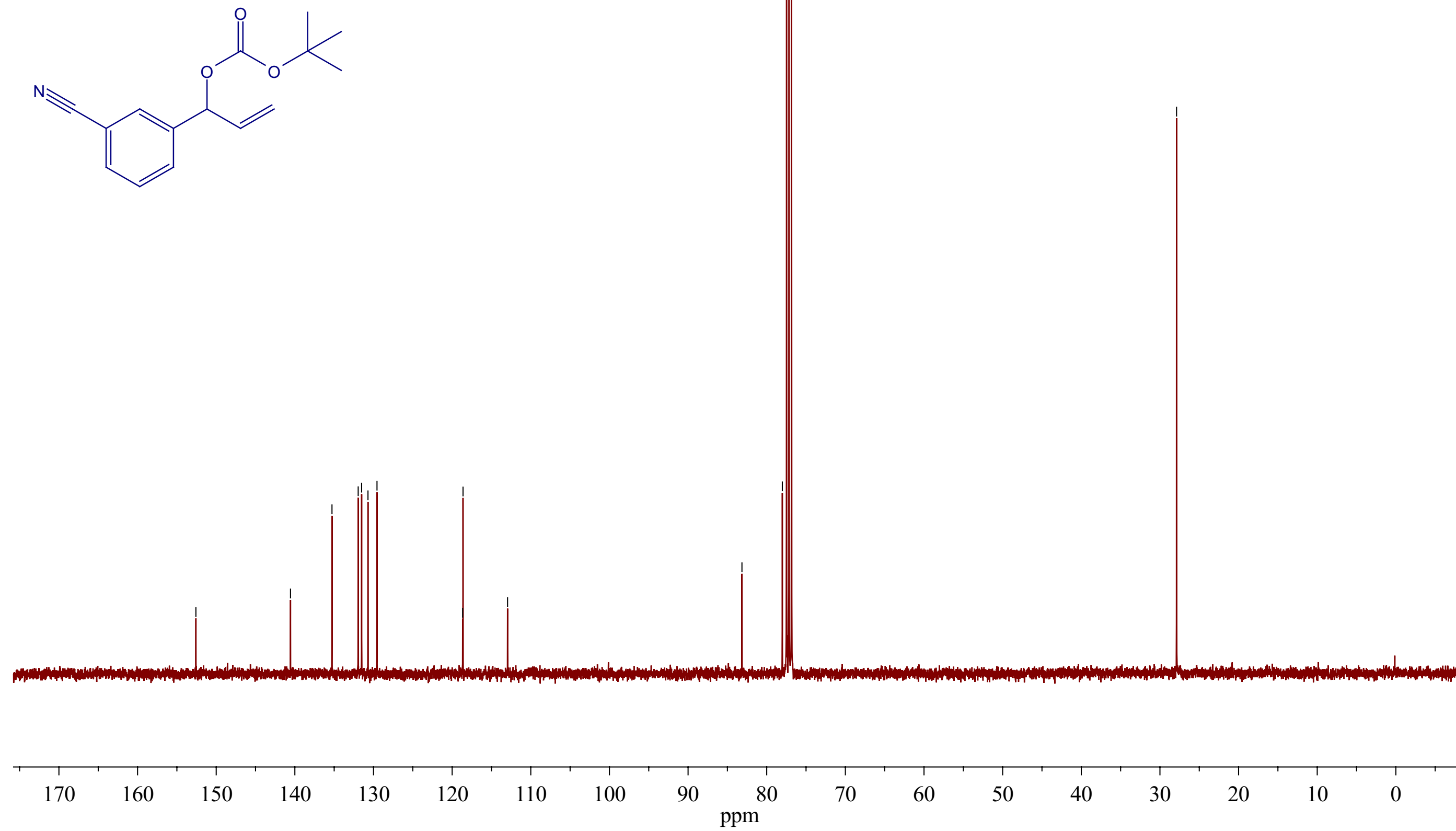
in $\mathrm{CDCl}_{3}, 400 \mathrm{MHz}$
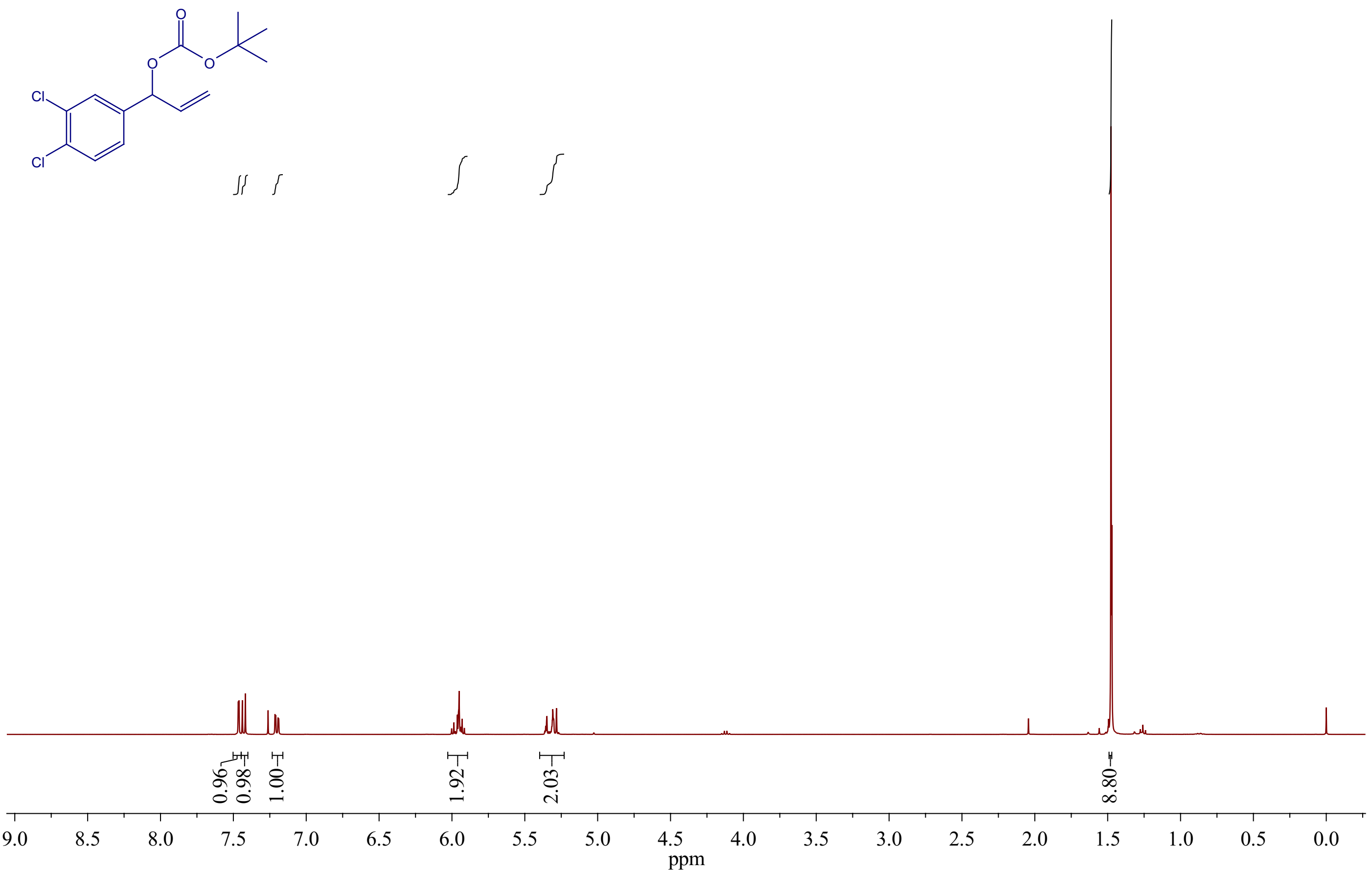


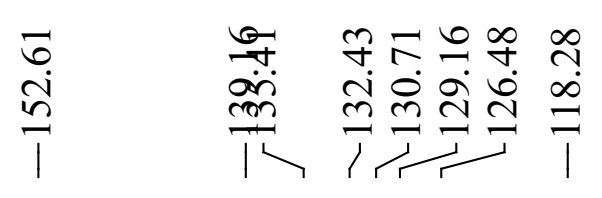

$\begin{array}{ll}\overline{0} & \hat{N} \\ \infty & \hat{\gamma} \\ 1 & 1\end{array}$

in $\mathrm{CDCl}_{3}, 101 \mathrm{MHz}$
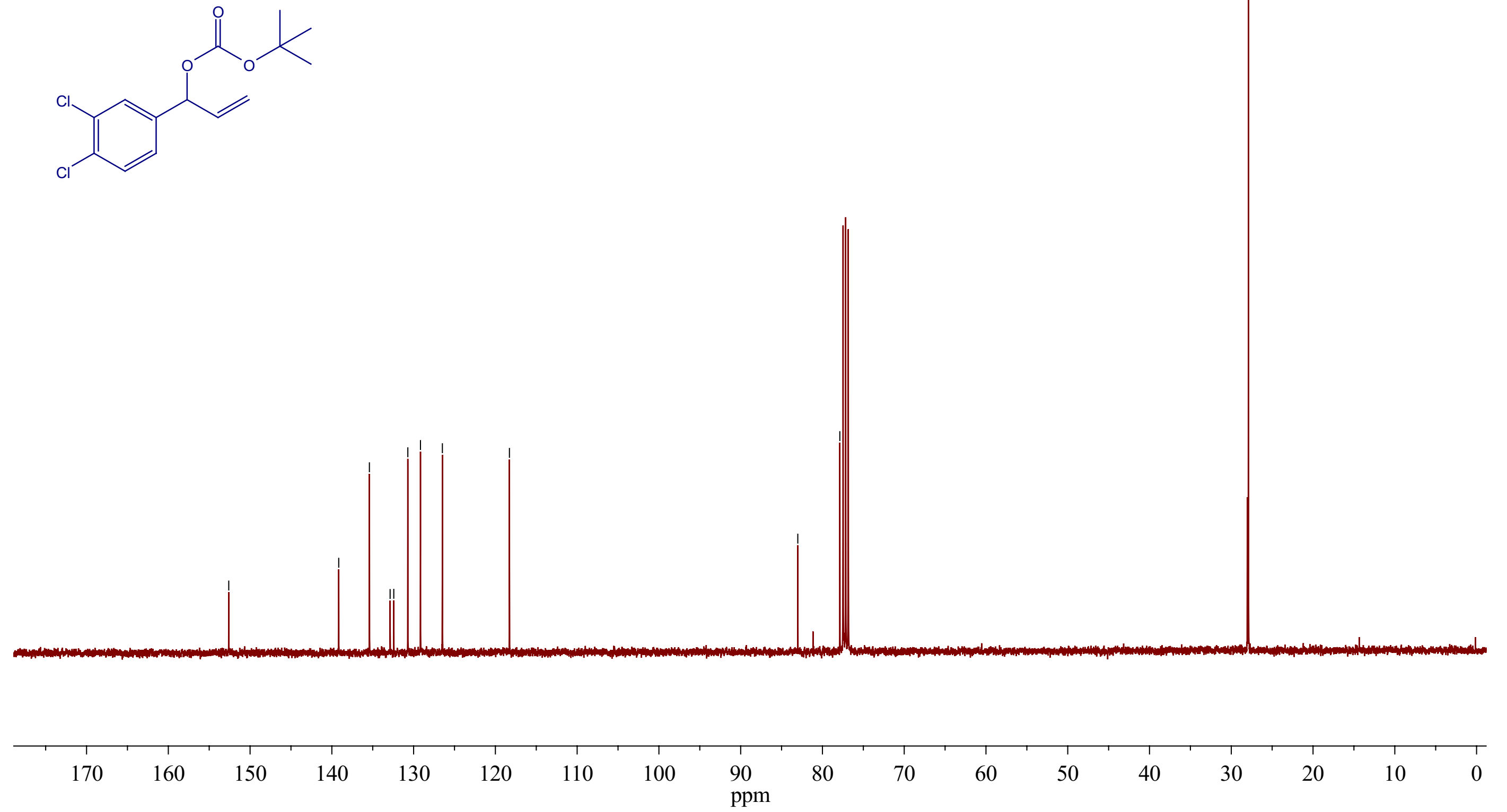


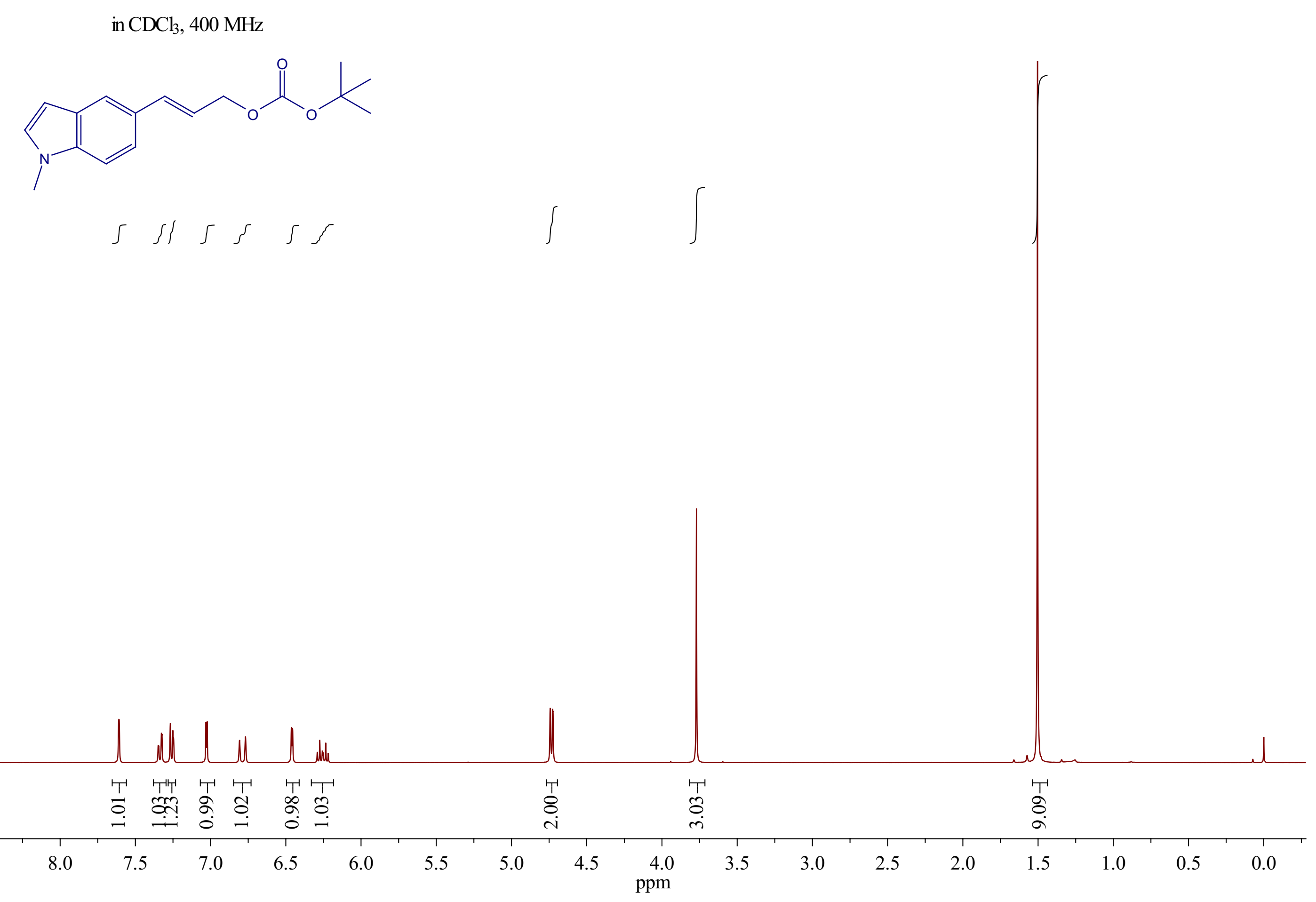




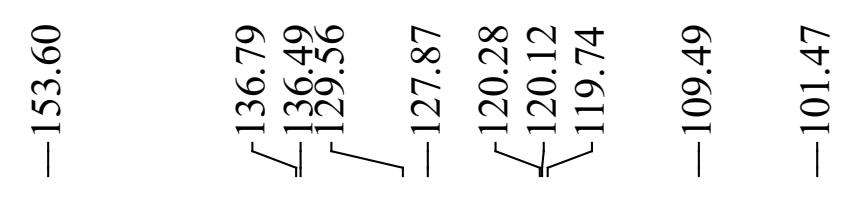

\begin{tabular}{ll}
$\infty$ & 0 \\
\multicolumn{1}{c}{} & $\infty$ \\
$\infty$ & $\infty$ \\
$\infty$ & 0 \\
0 & 1
\end{tabular}

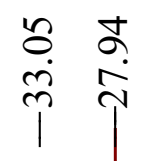

in $\mathrm{CDCl}_{3}, 101 \mathrm{MHz}$

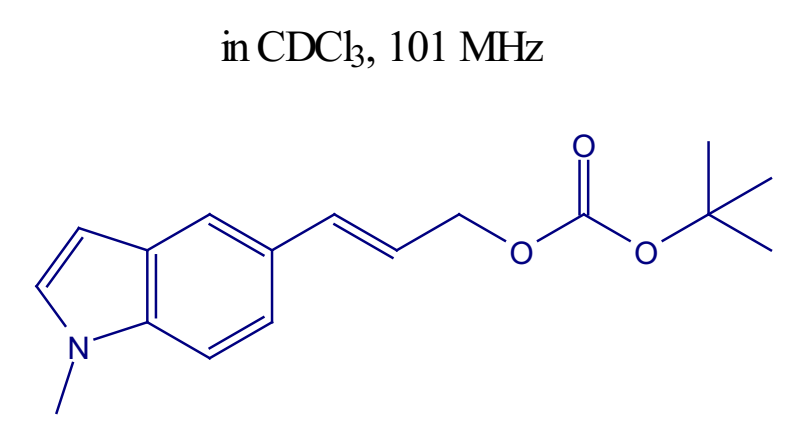

象 
in $\mathrm{CDCl}_{3}, 400 \mathrm{MHz}$
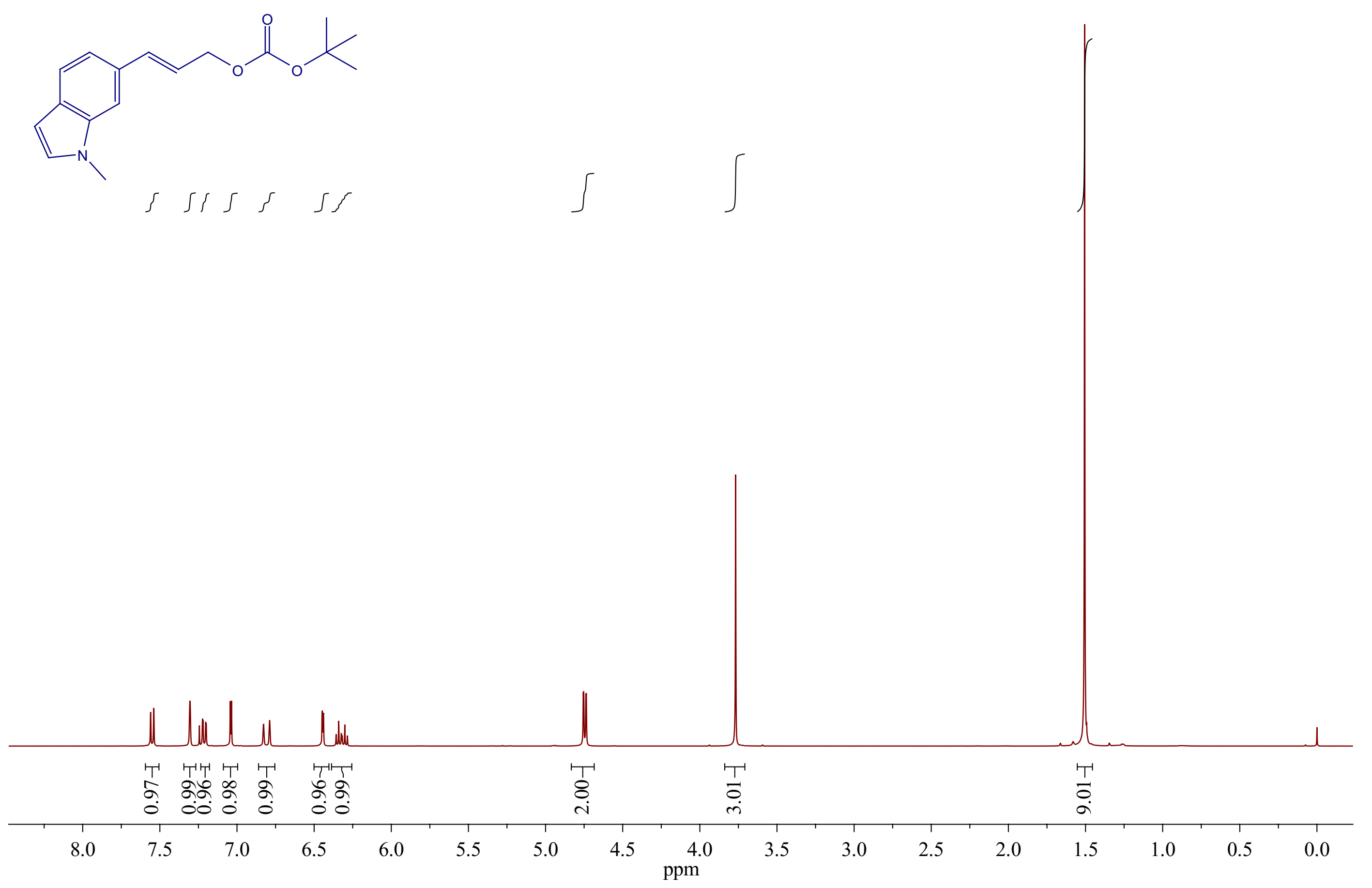


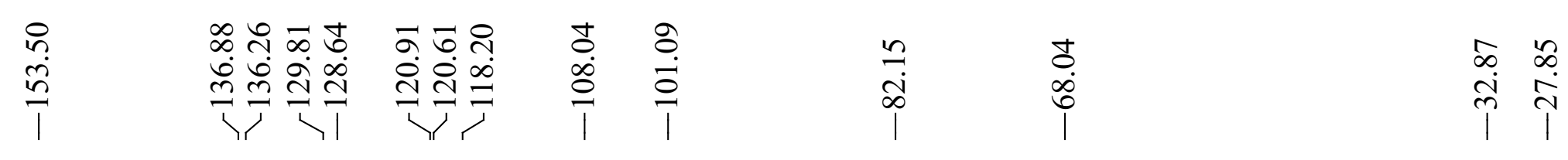

in $\mathrm{CDCl}_{3}, 101 \mathrm{MHz}$
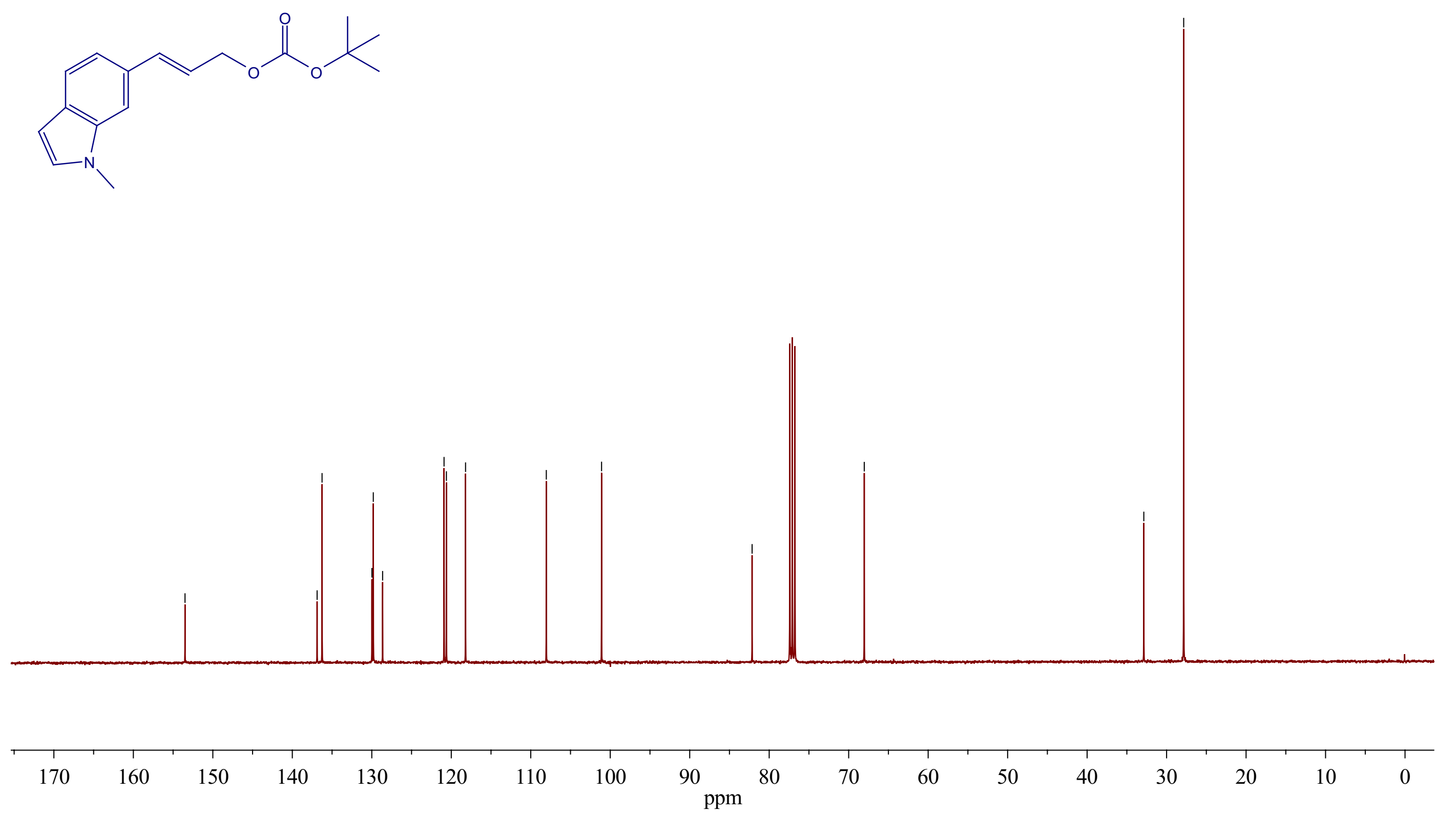
in $\mathrm{CDCl}_{3}, 400 \mathrm{MHz}$
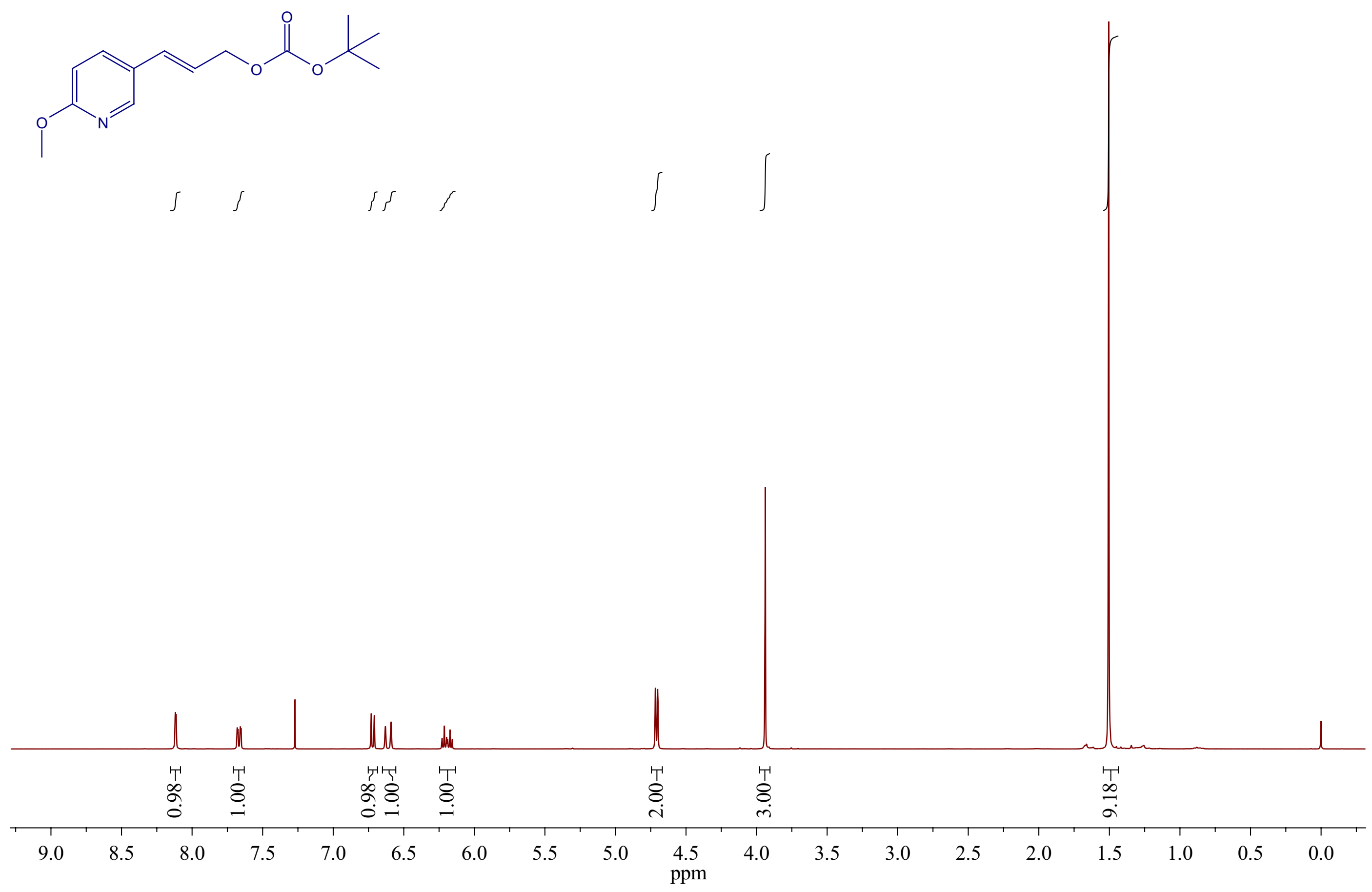
in $\mathrm{CDCl}_{3}, 101 \mathrm{MHz}$
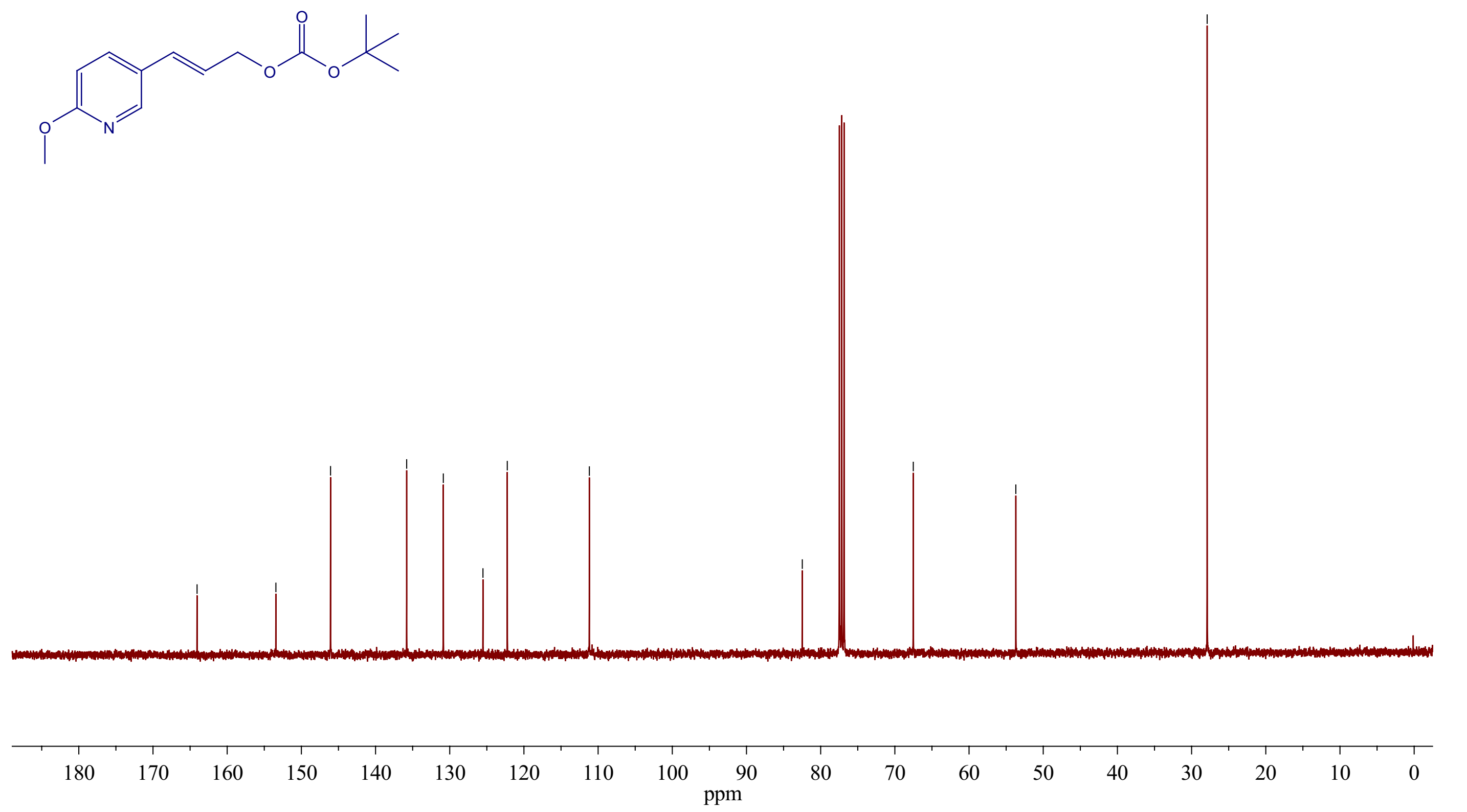


\section{in $\mathrm{CDCB}, 400 \mathrm{MHz}$}
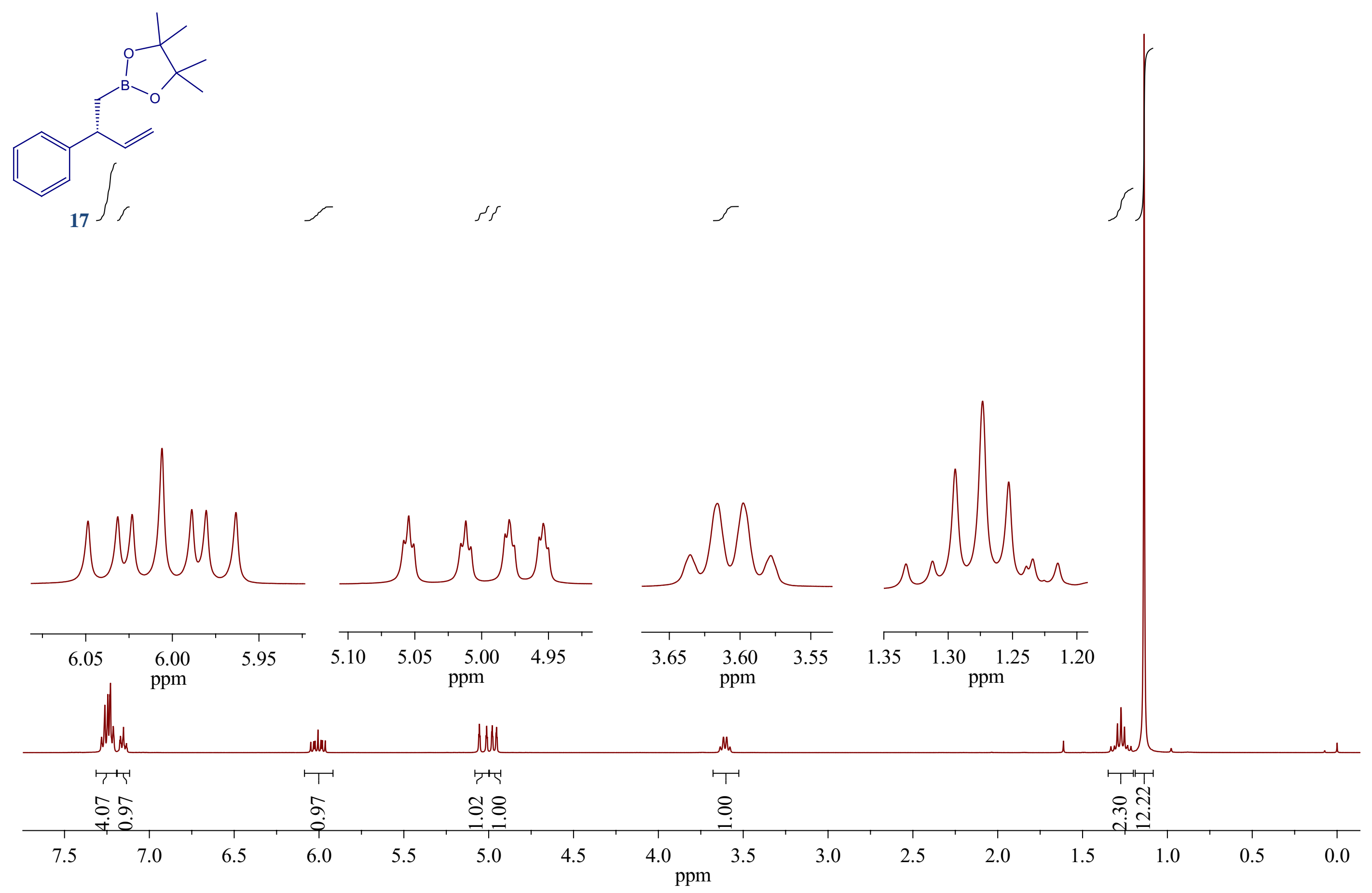

앙
守守

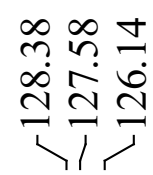
$\stackrel{8}{i}$

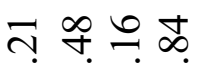
क숭요

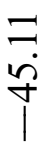
$\infty$
$\stackrel{\infty}{+}$
$\stackrel{+}{\sim}$

in $\mathrm{CDCl}_{3}, 101 \mathrm{MHz}$

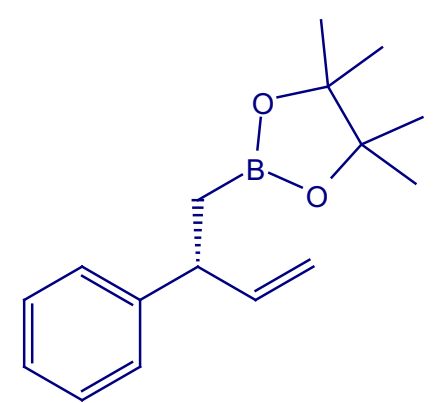

17

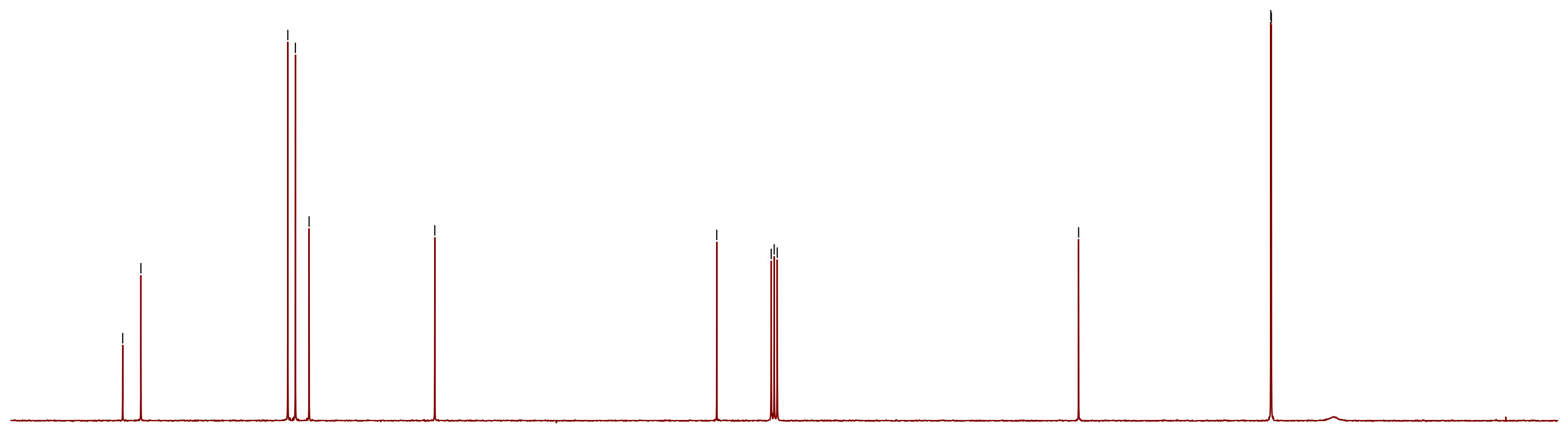


in $\mathrm{CDCl} 3,400 \mathrm{MHz}$

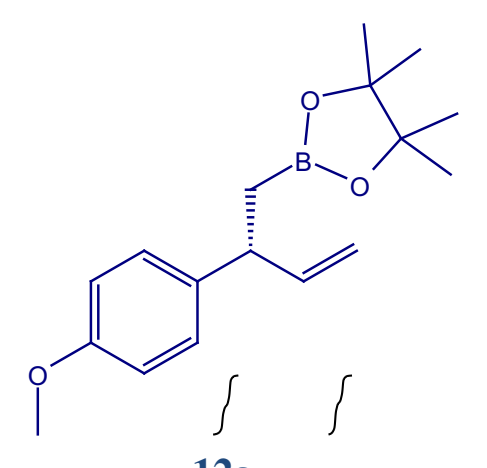

S

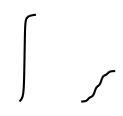

$\int$

12a

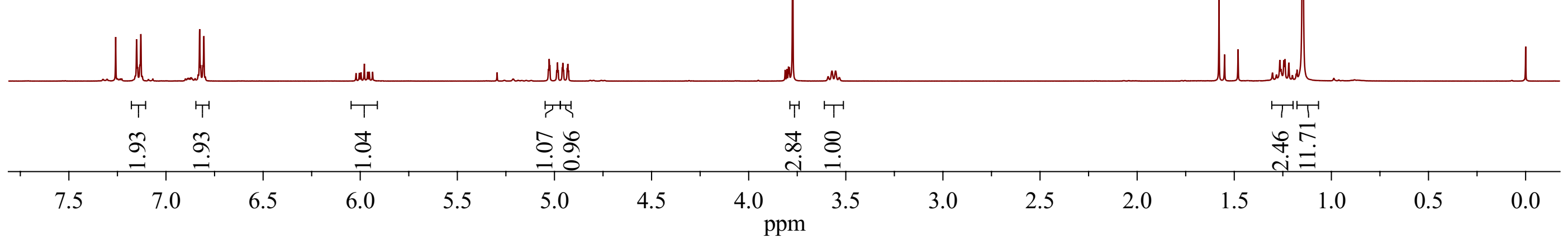




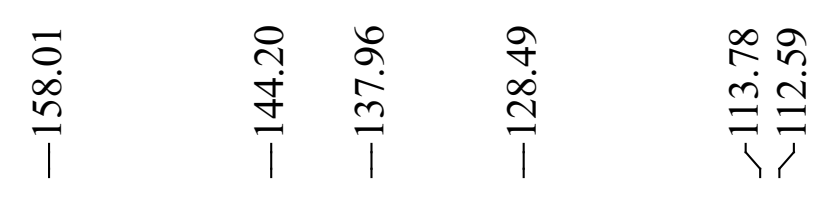

in $\mathrm{CDCl}_{3}, 101 \mathrm{MHz}$
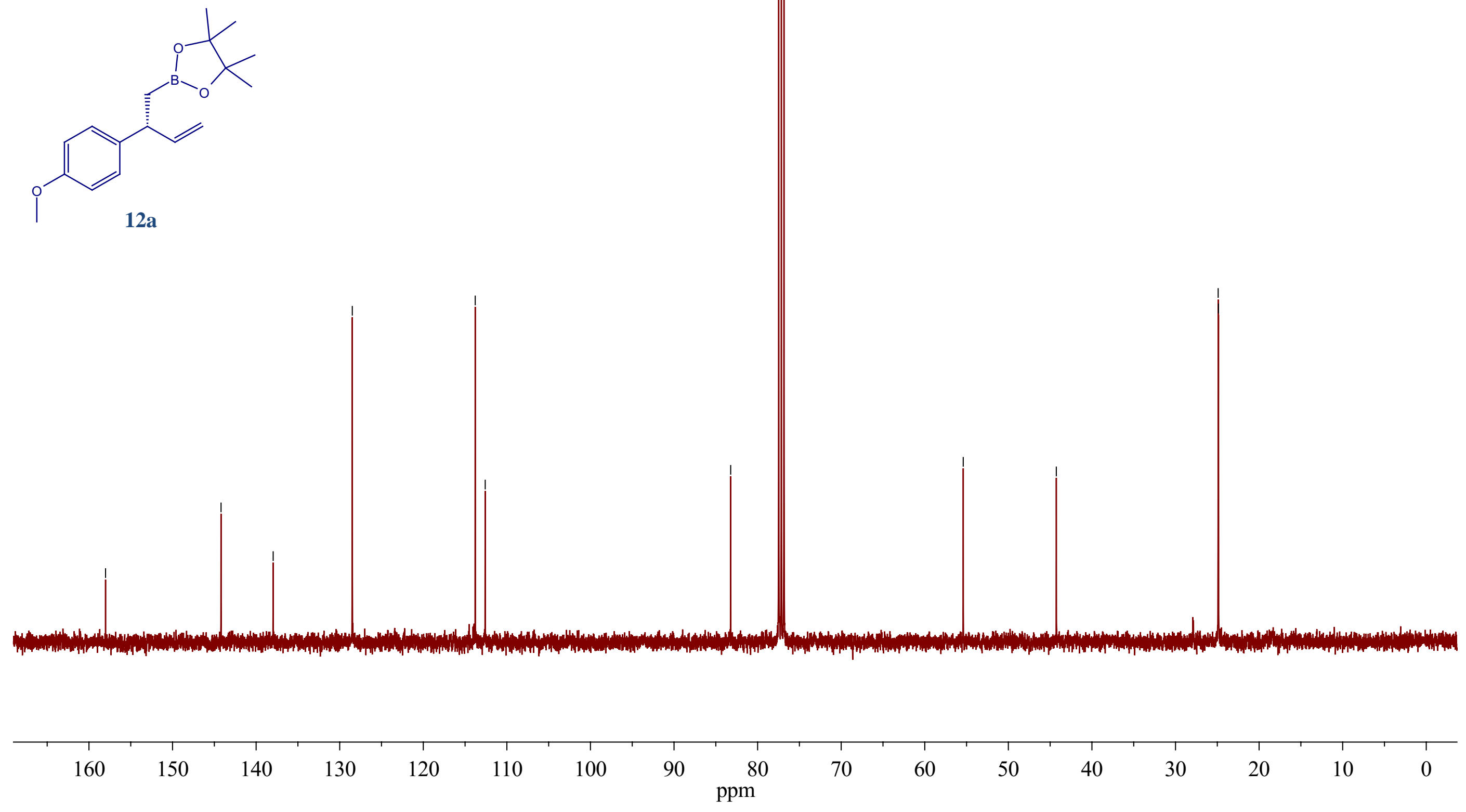


\section{in $\mathrm{CDCB}, 400 \mathrm{MHz}$}
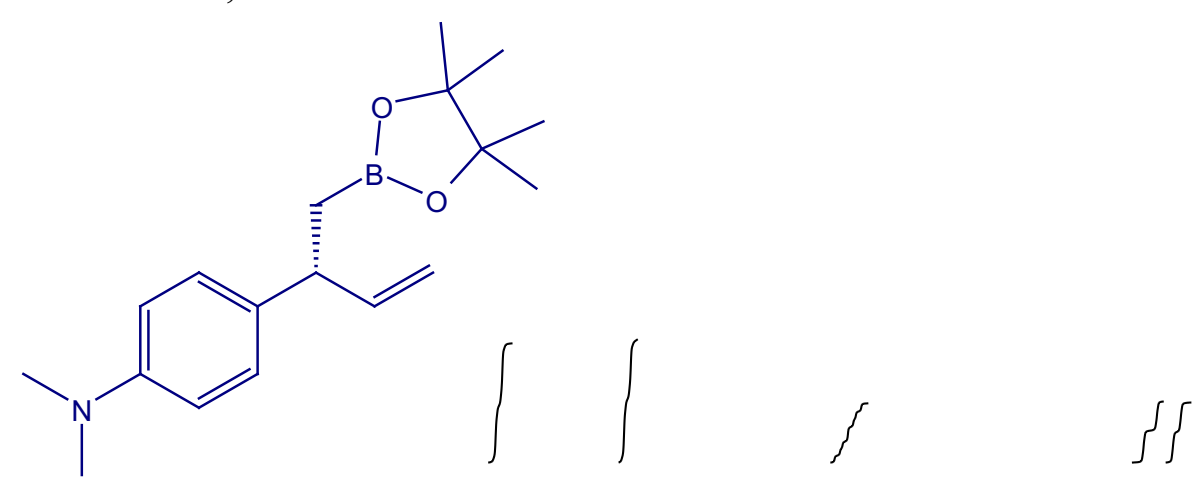

12b

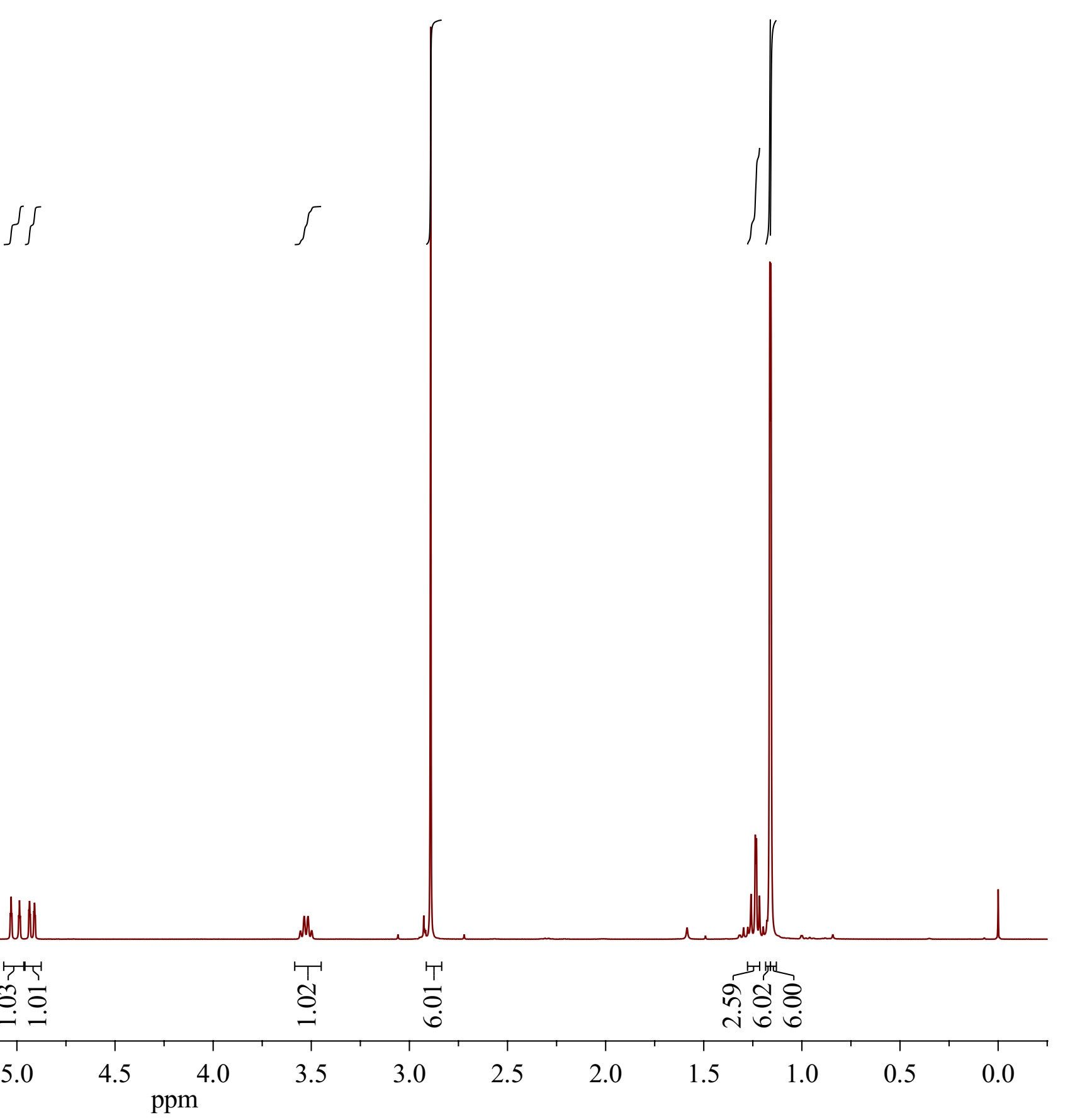




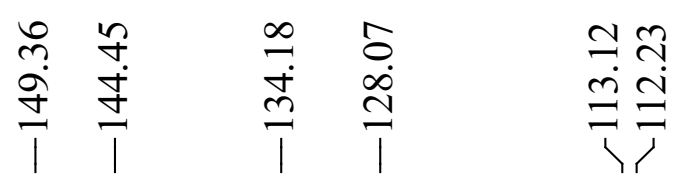
$\stackrel{n}{\infty}$

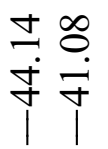

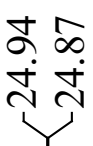

in $\mathrm{CDCB}, 101 \mathrm{MHz}$

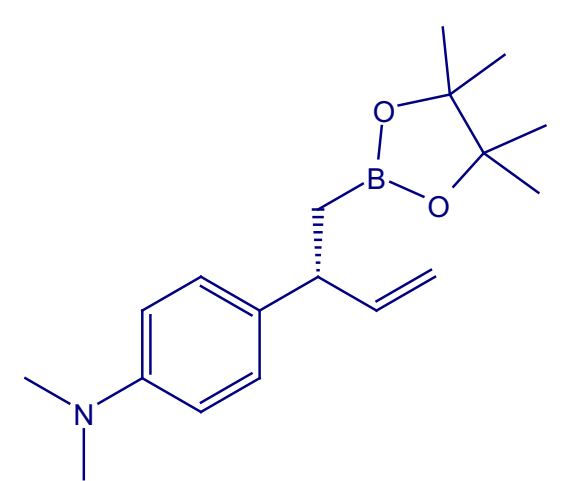

12b

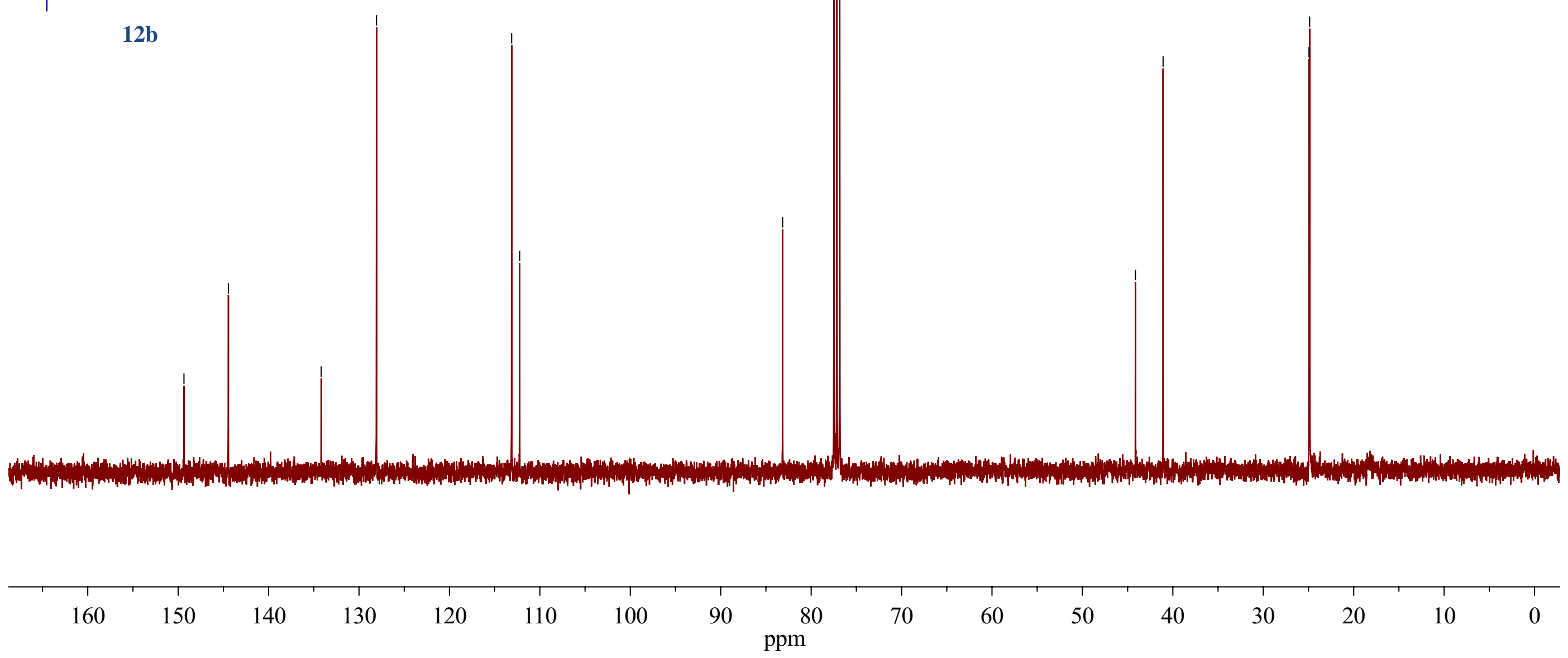


in $\mathrm{CDCB}, 400 \mathrm{MHz}$

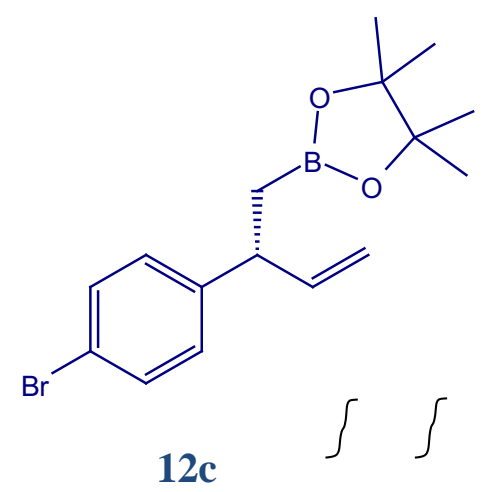

$12 \mathrm{c}$

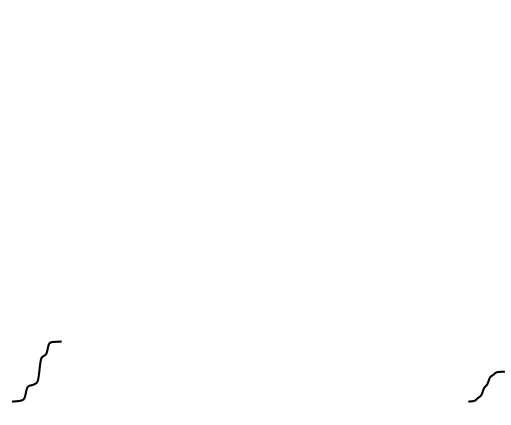

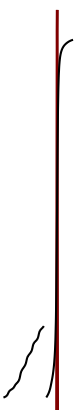

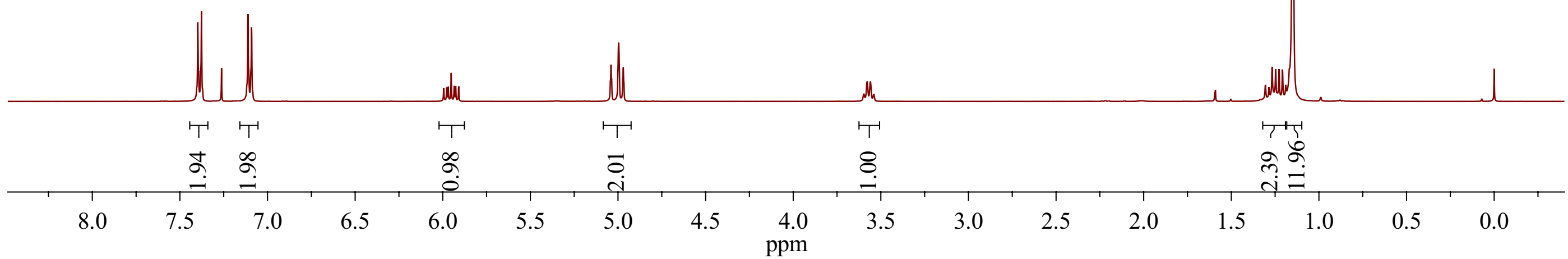




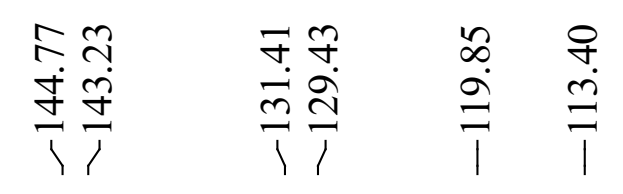

$\dot{H}$
$\infty$
$\infty$
$\infty$

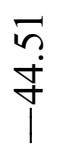

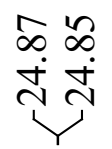

in $\mathrm{CDCB}, 101 \mathrm{MHz}$

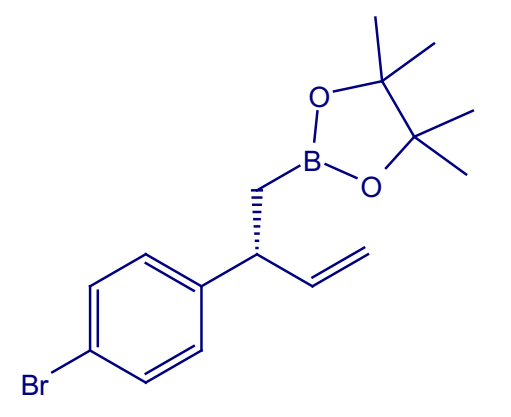

$12 \mathrm{c}$
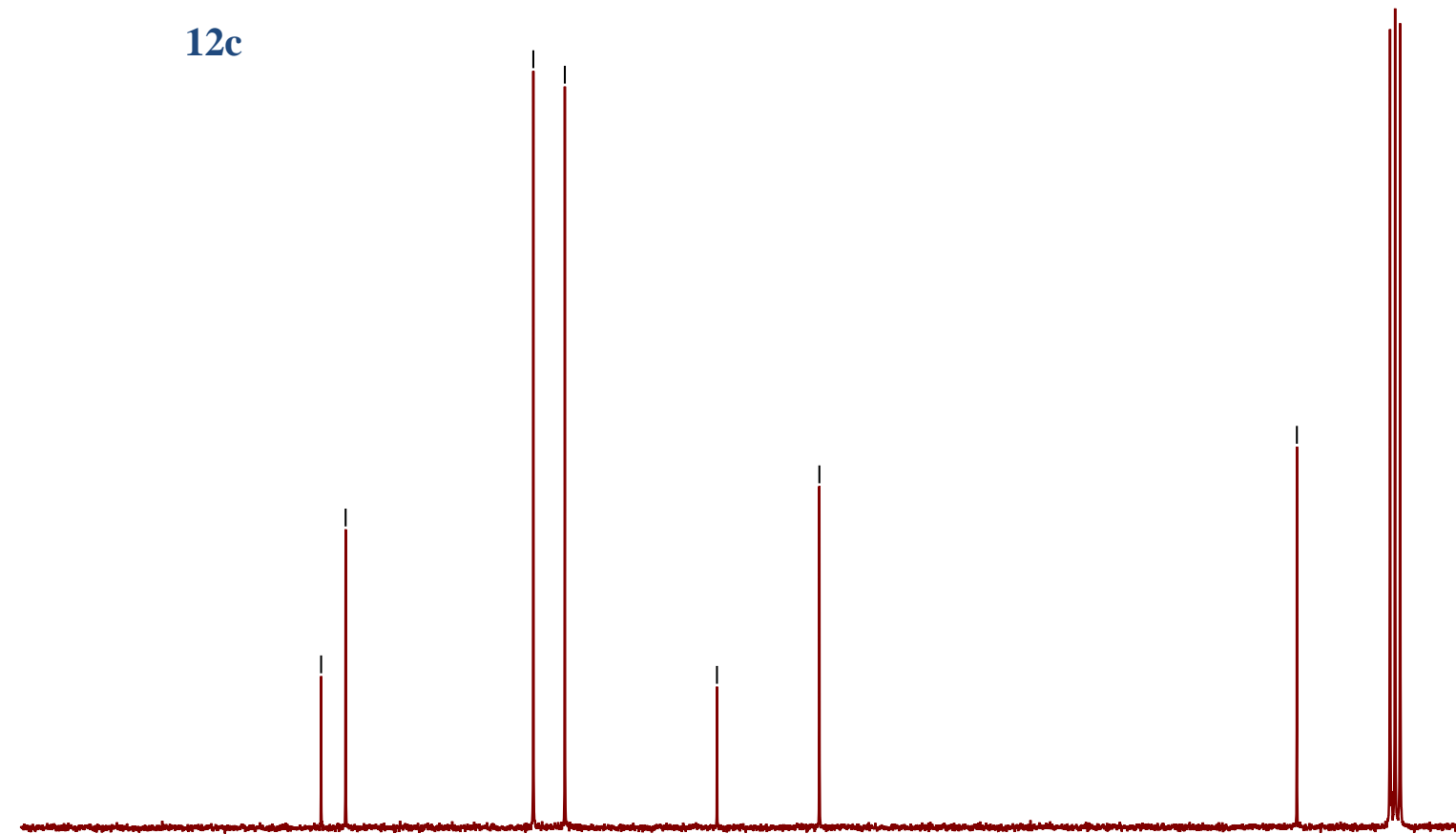


\section{in $\mathrm{CDCl}$, $400 \mathrm{MHz}$}

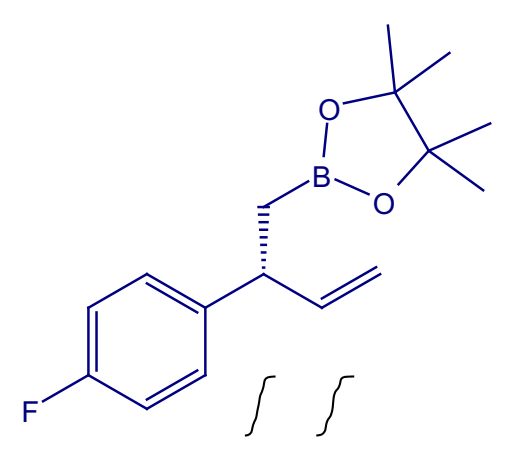

r

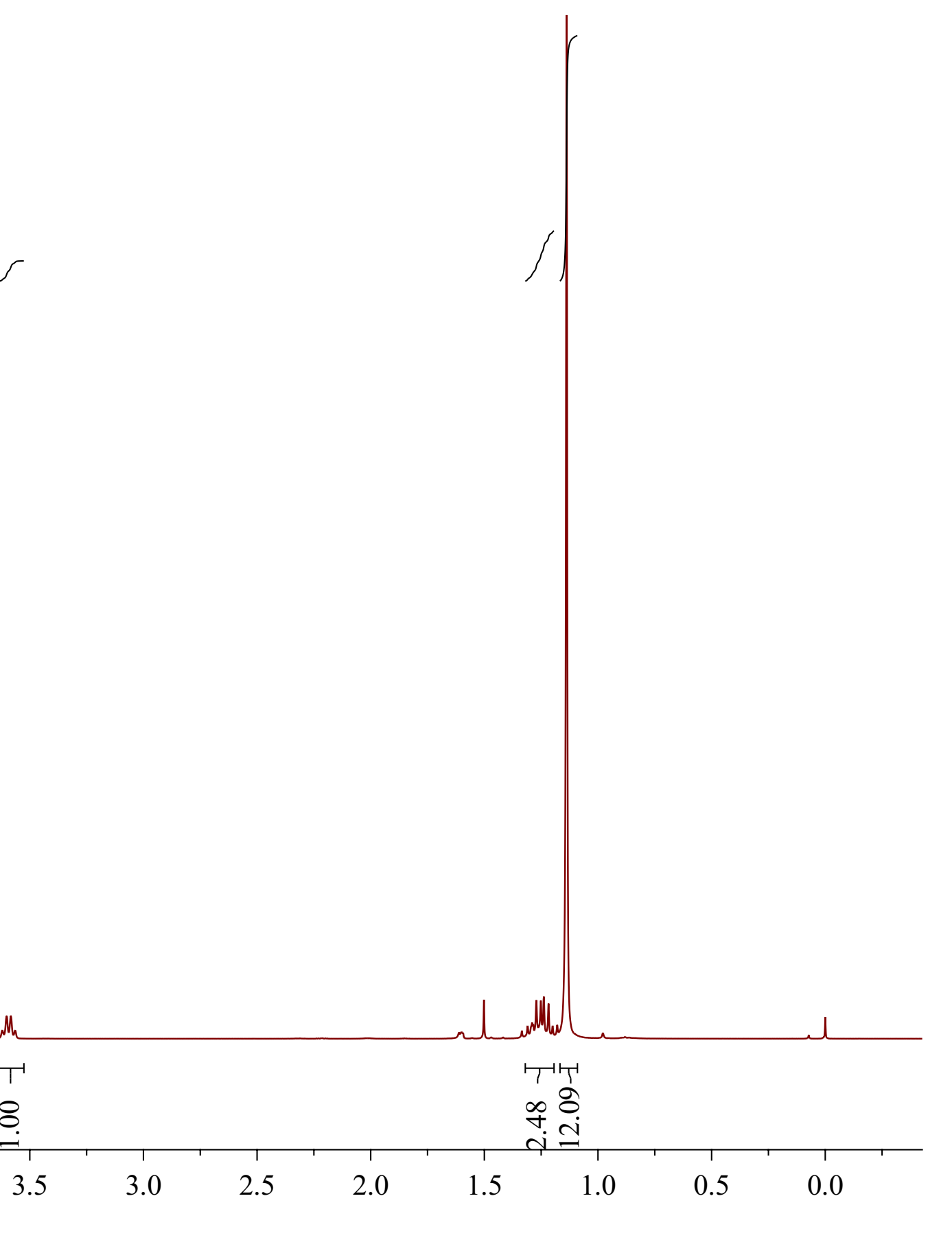




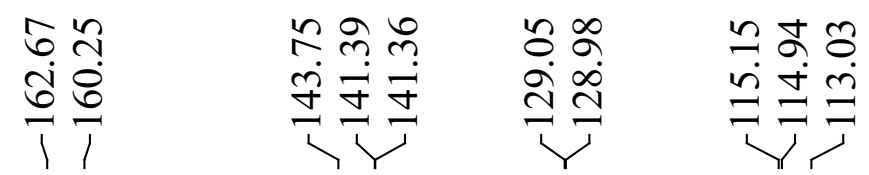

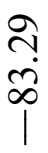

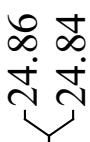

\section{in $\mathrm{CDCl}_{3}, 101 \mathrm{MHz}$}

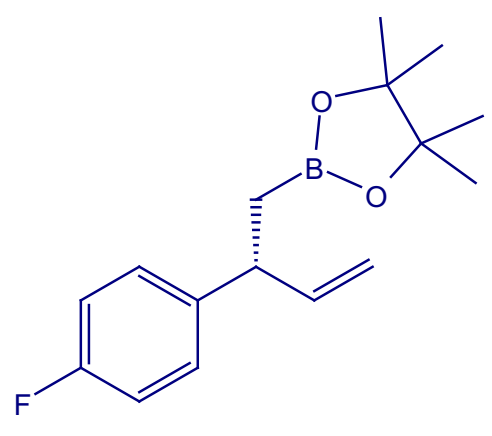

12d
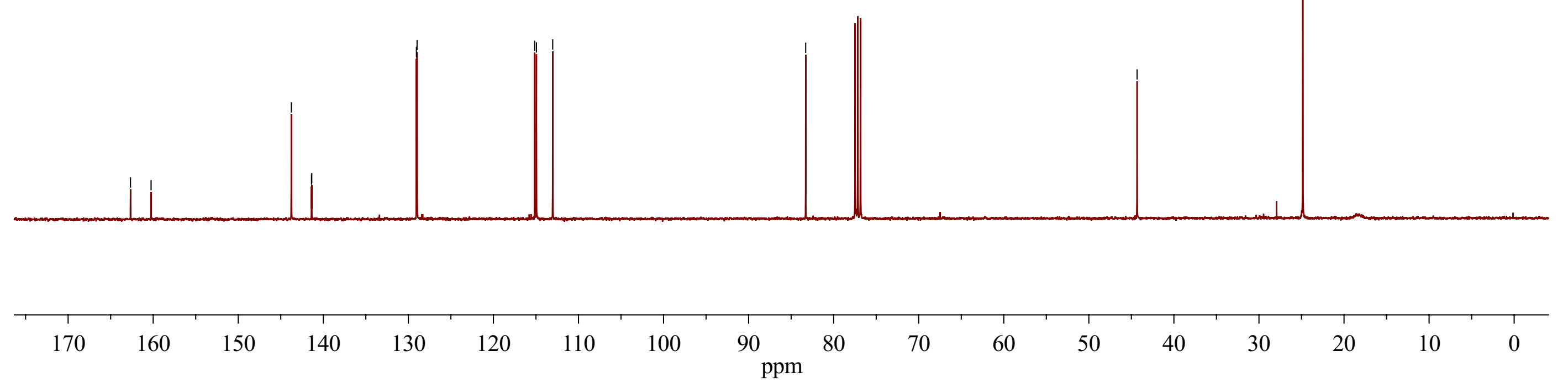

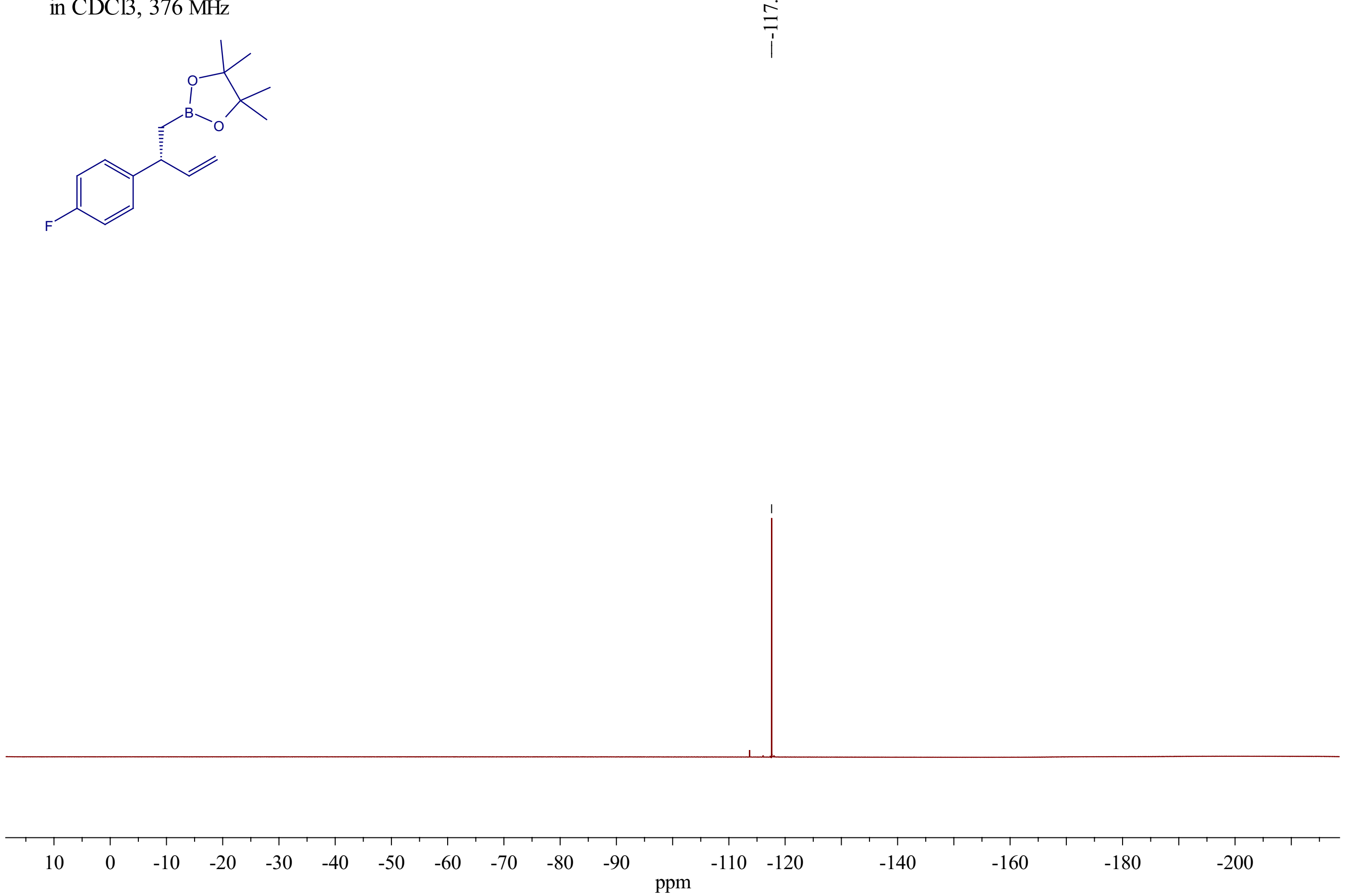
in $\mathrm{CDCl}_{3}, 400 \mathrm{MHz}$
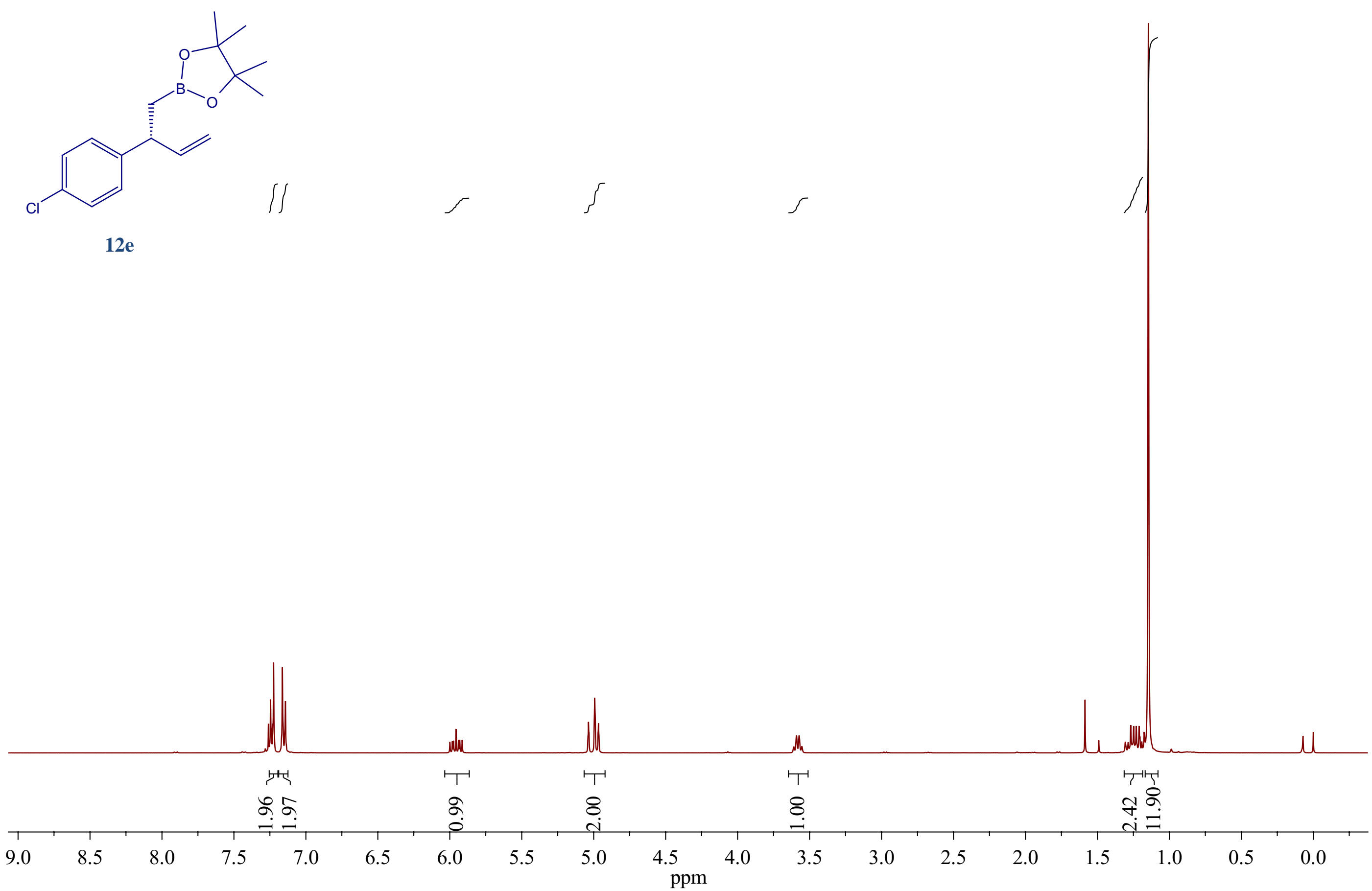


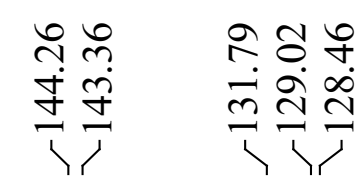

$\stackrel{m}{m}$

in $\mathrm{CDCl}_{3}, 101 \mathrm{MHz}$

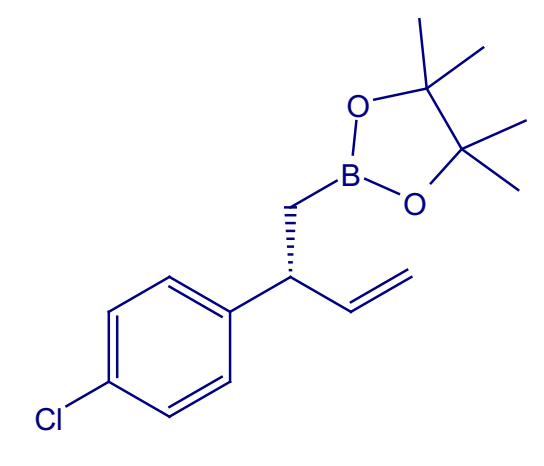

$12 \mathrm{e}$ $\stackrel{+}{\infty}$

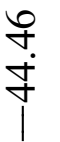

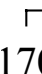

160

150

140

130

120

110

100

90 
in $\mathrm{CDCB}$, $400 \mathrm{MHz}$
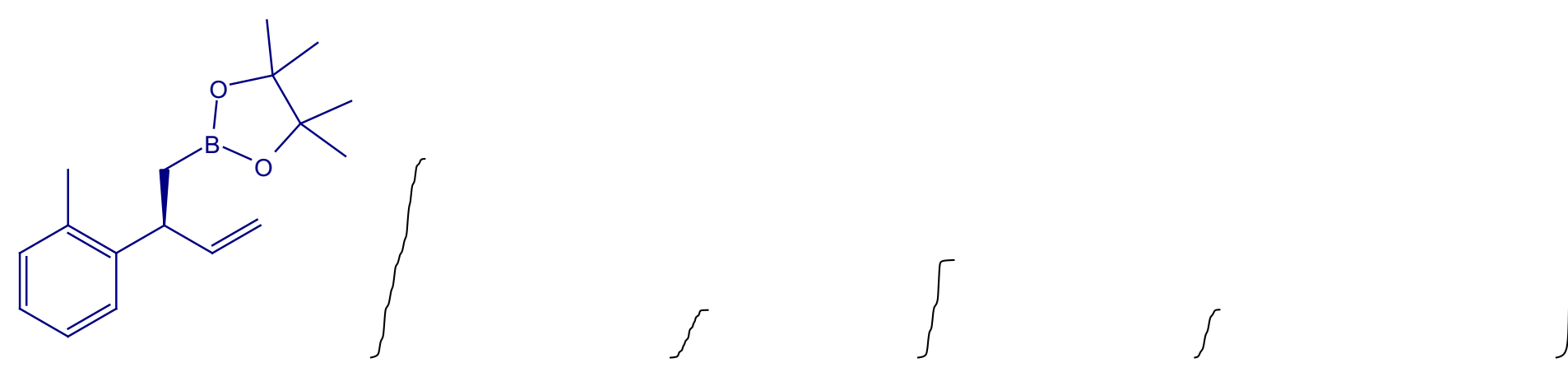

$12 f$
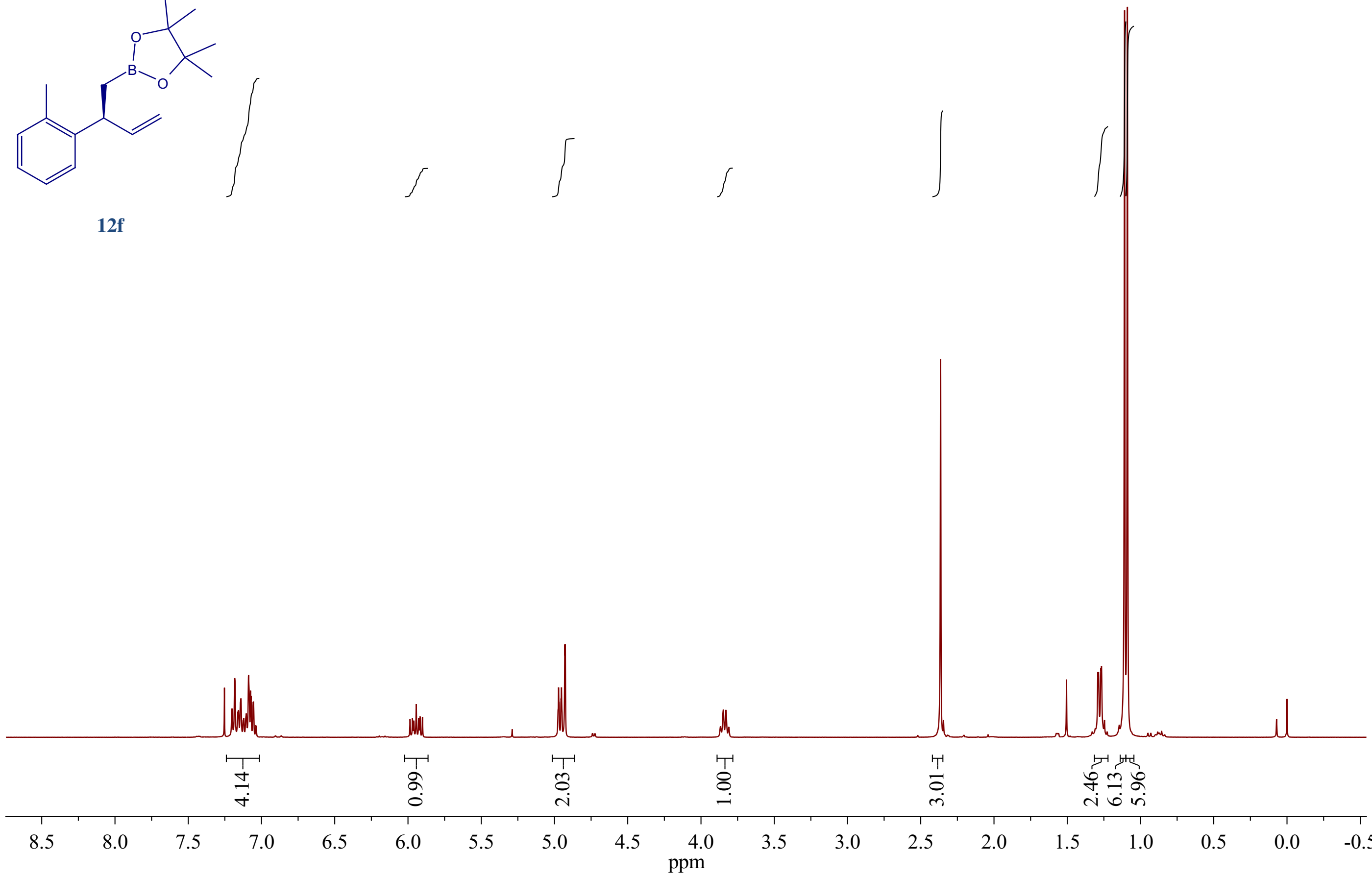
in CDCB, $101 \mathrm{MHz}$

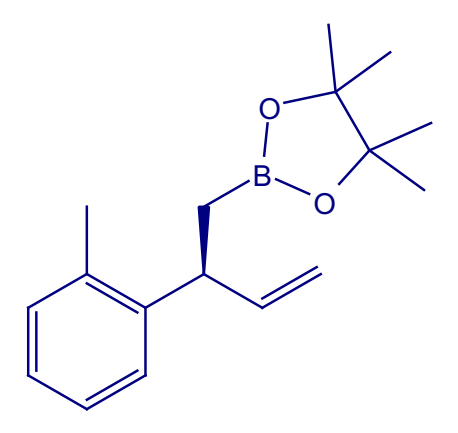

$12 \mathrm{f}$

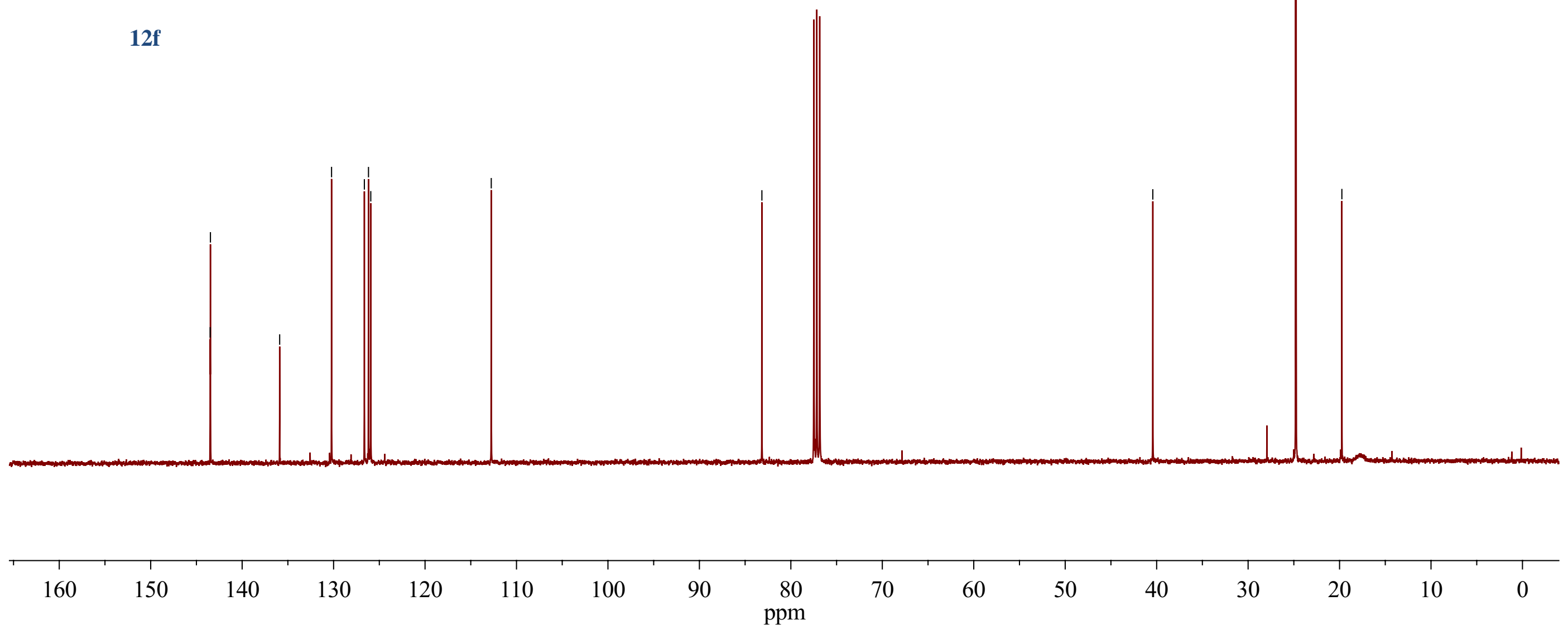




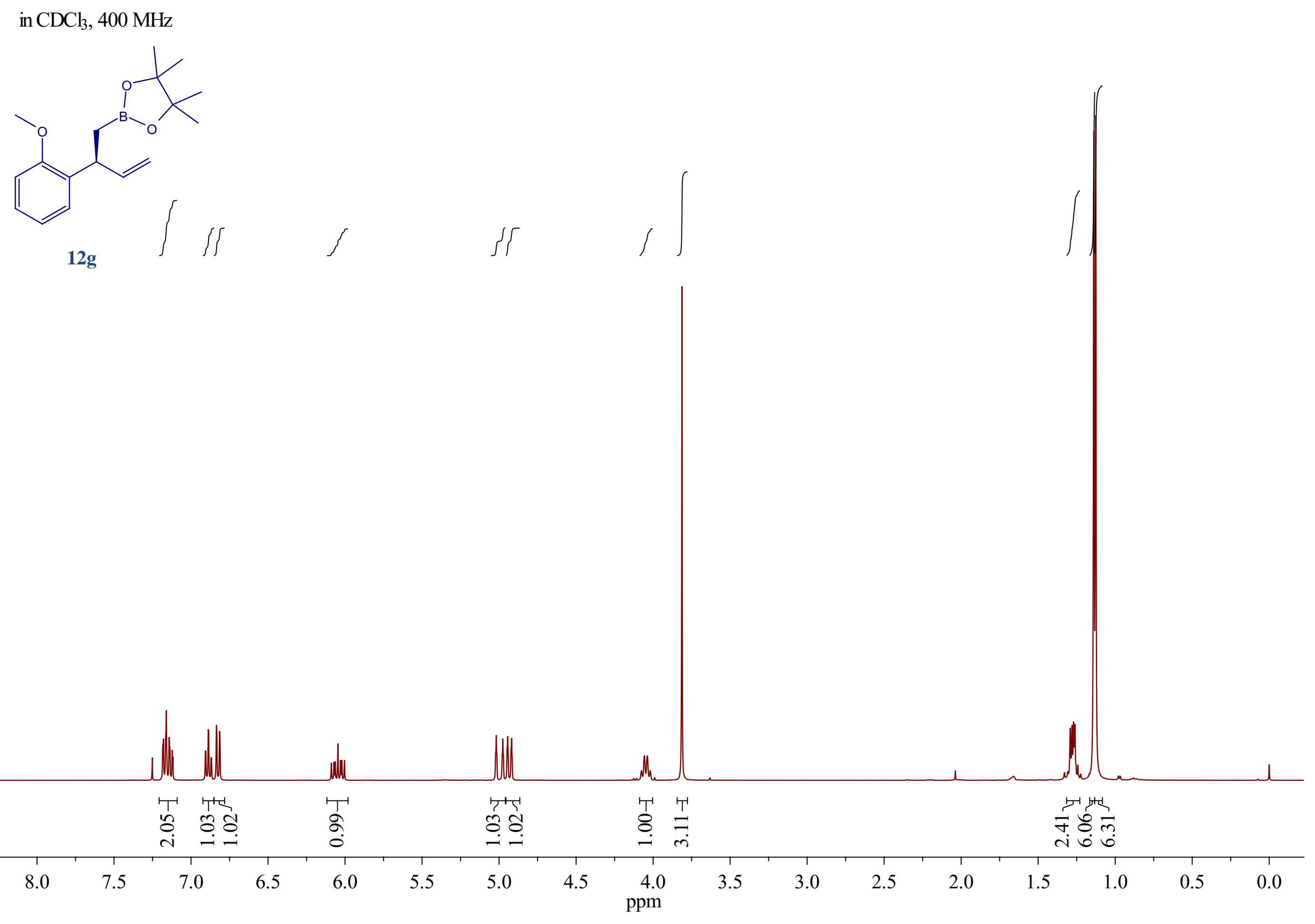




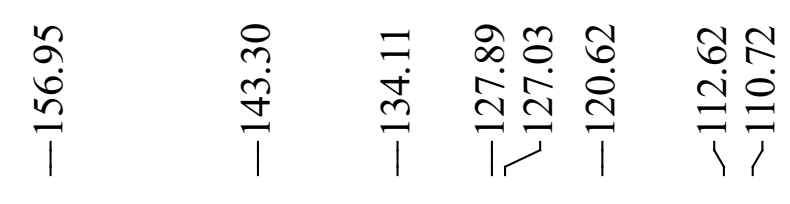

$\underset{\substack{2 \\ \infty \\ \infty \\ \infty}}{0}$

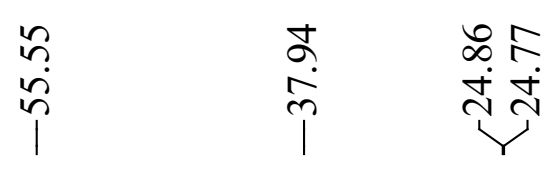

in $\mathrm{CDCl}_{3}, 101 \mathrm{MHz}$

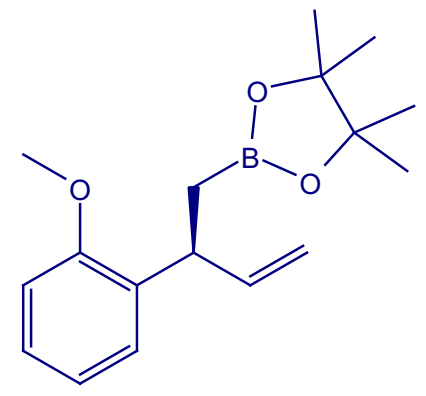

$12 \mathrm{~g}$

i $\quad$ in

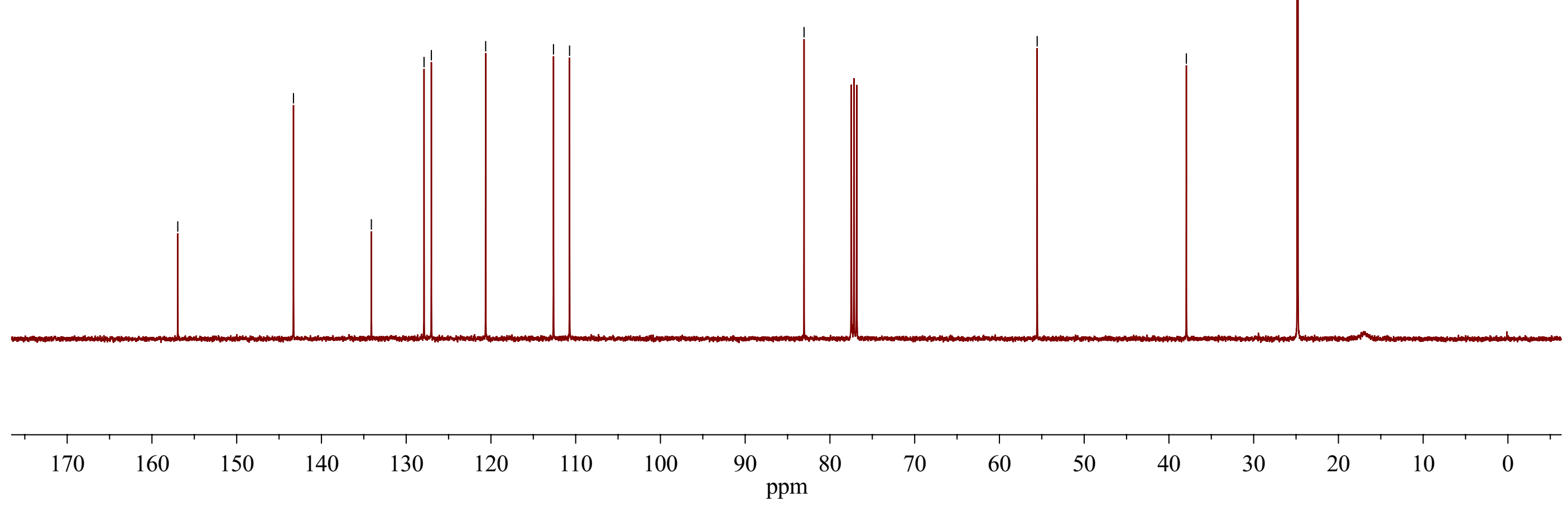




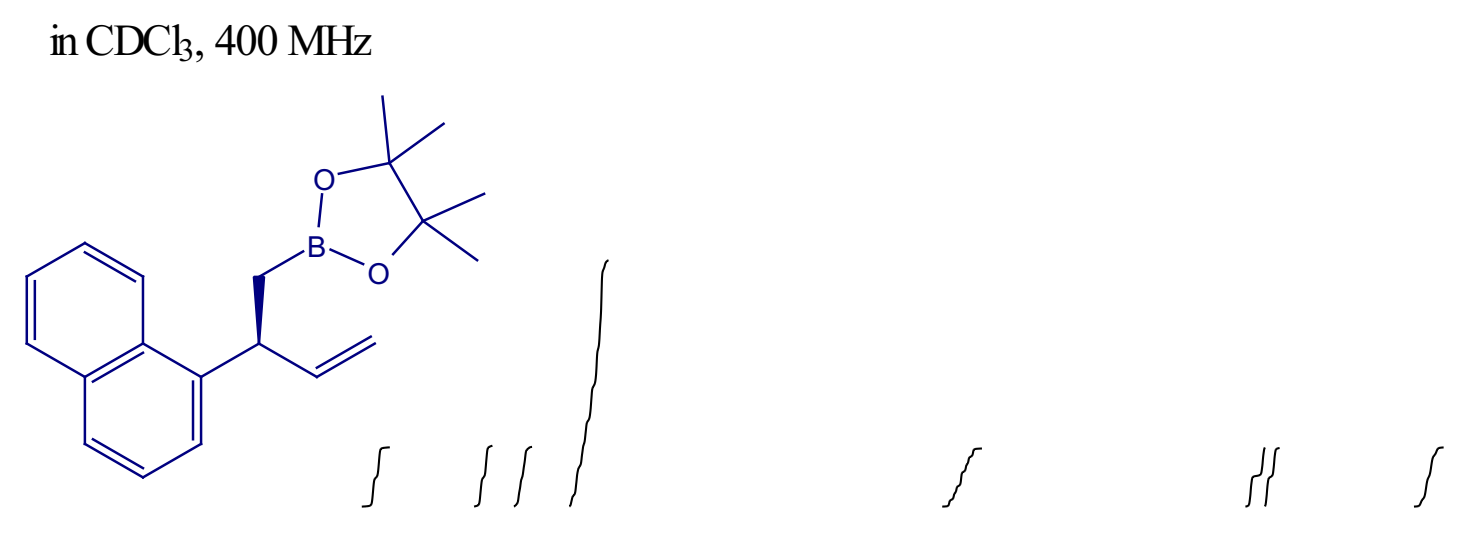

$12 \mathrm{~h}$

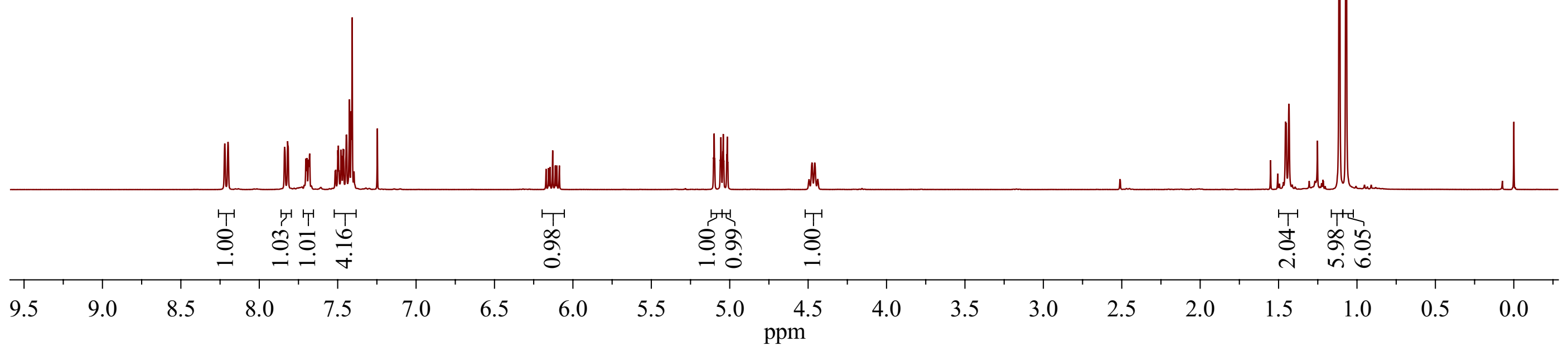




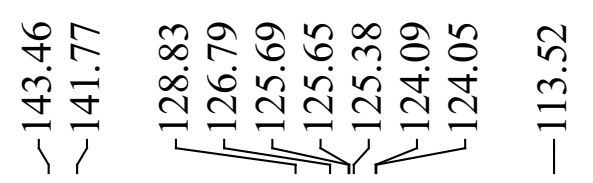

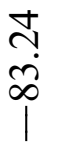

ลั๋

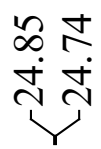

in $\mathrm{CDCl}_{3}, 101 \mathrm{MHz}$

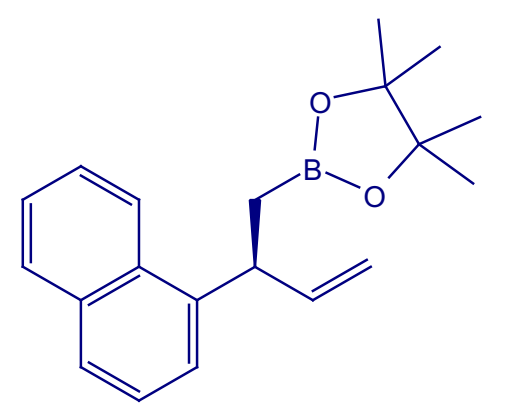

$12 \mathrm{~h}$

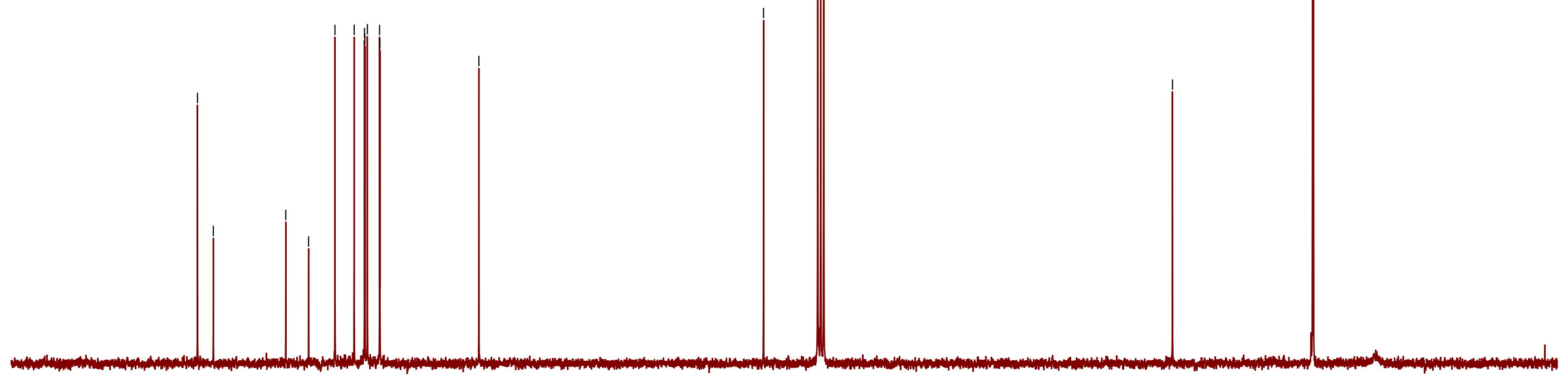


in $\mathrm{CDCl}_{3}, 400 \mathrm{MHz}$

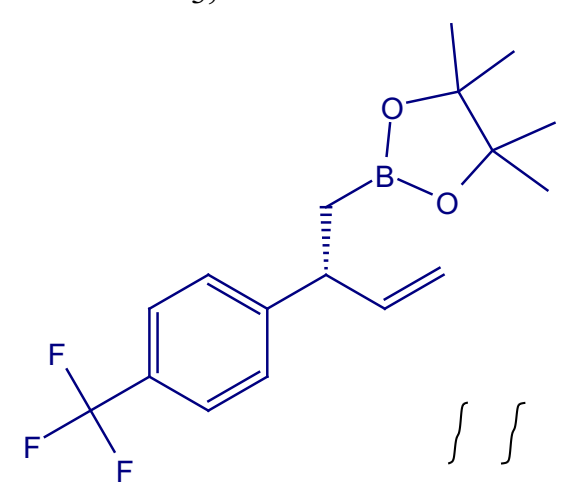

$12 \mathrm{i}$

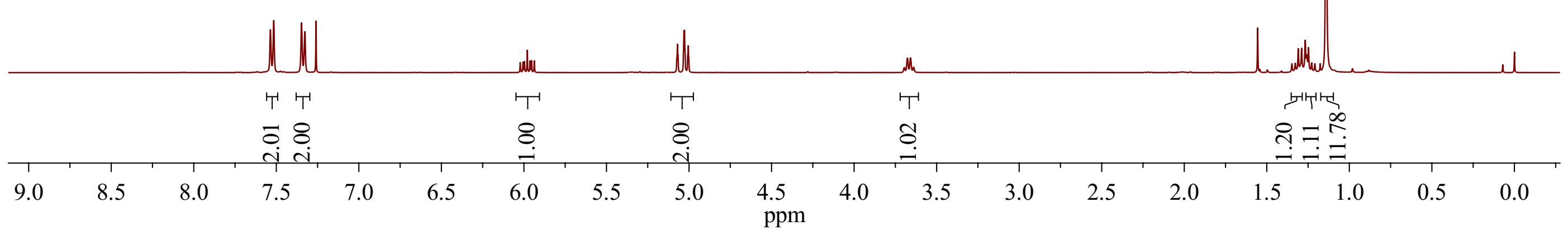




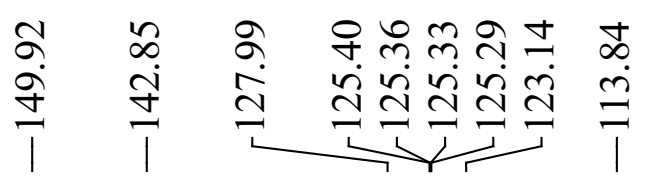

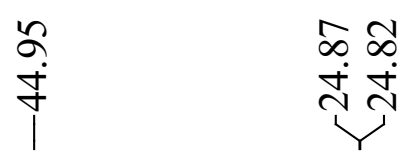

in $\mathrm{CDCl}_{3}, 101 \mathrm{MHz}$

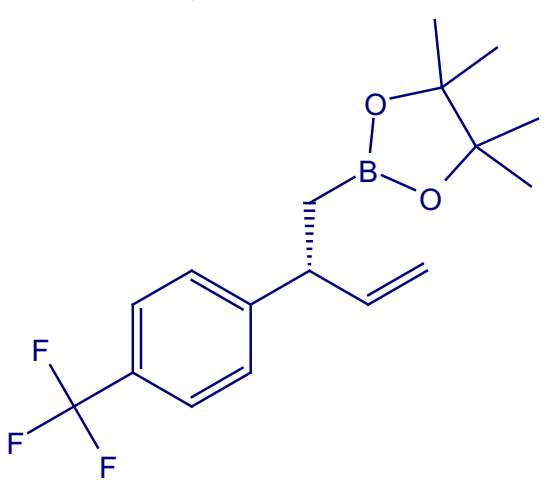

$12 \mathrm{i}$

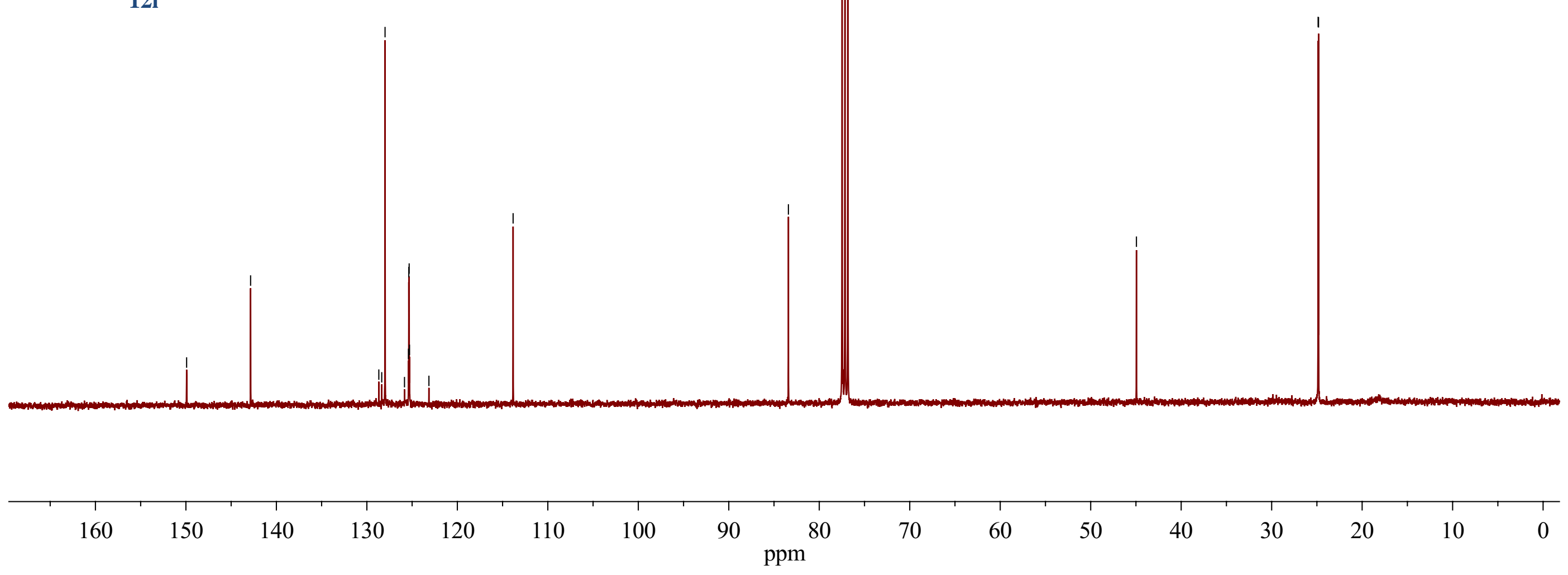



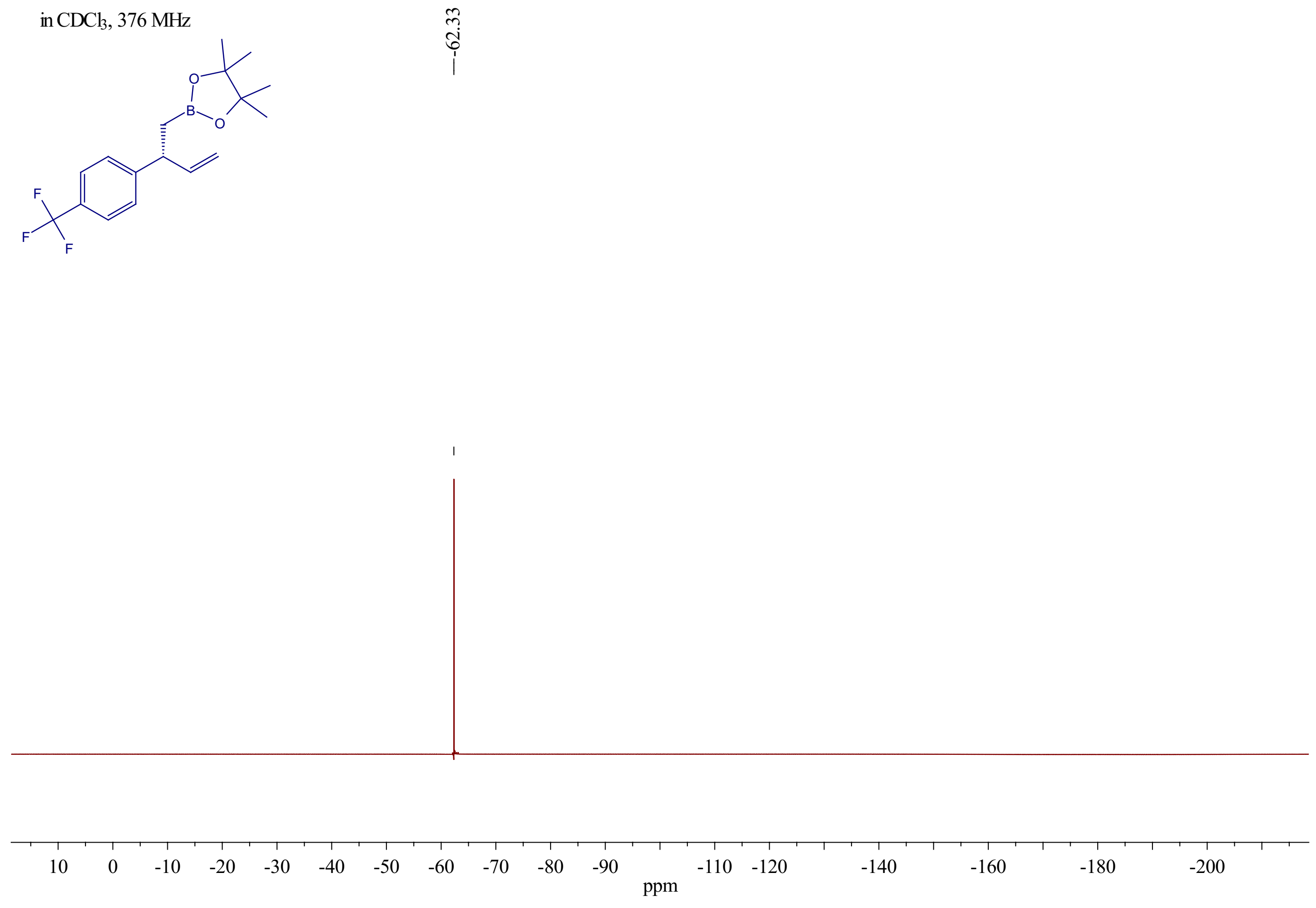
in $\mathrm{CDCl}_{3}, 400 \mathrm{MHz}$
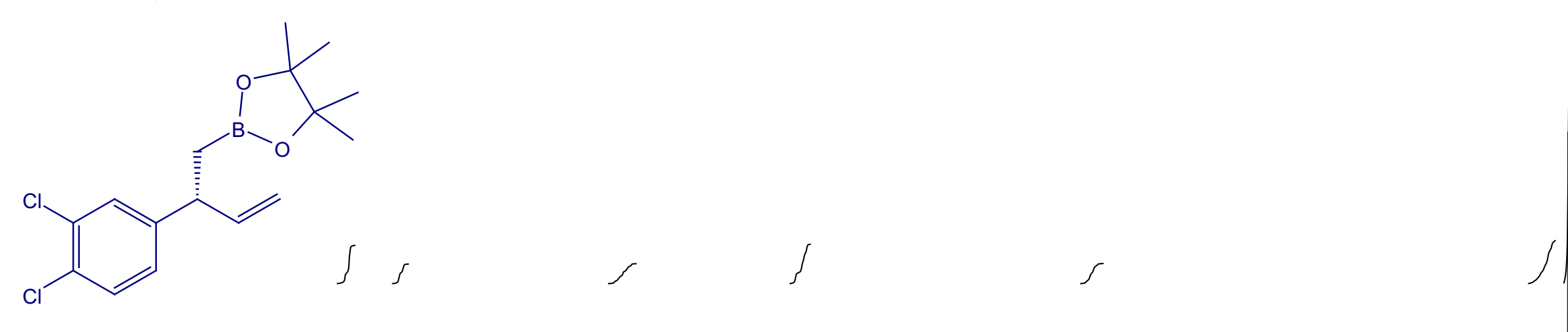

12j

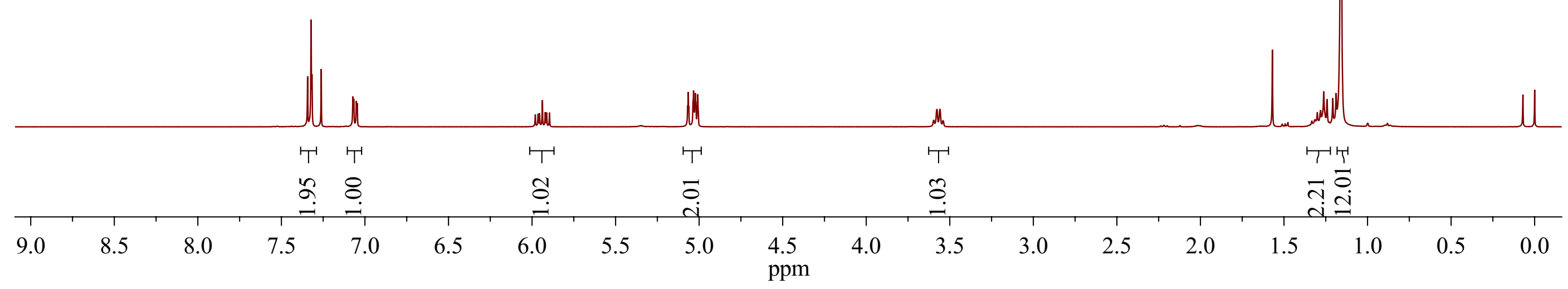


in $\mathrm{CDCl}_{3}, 101 \mathrm{MHz}$

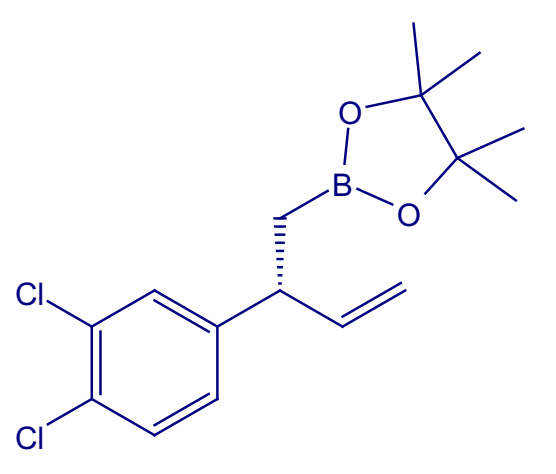

12j
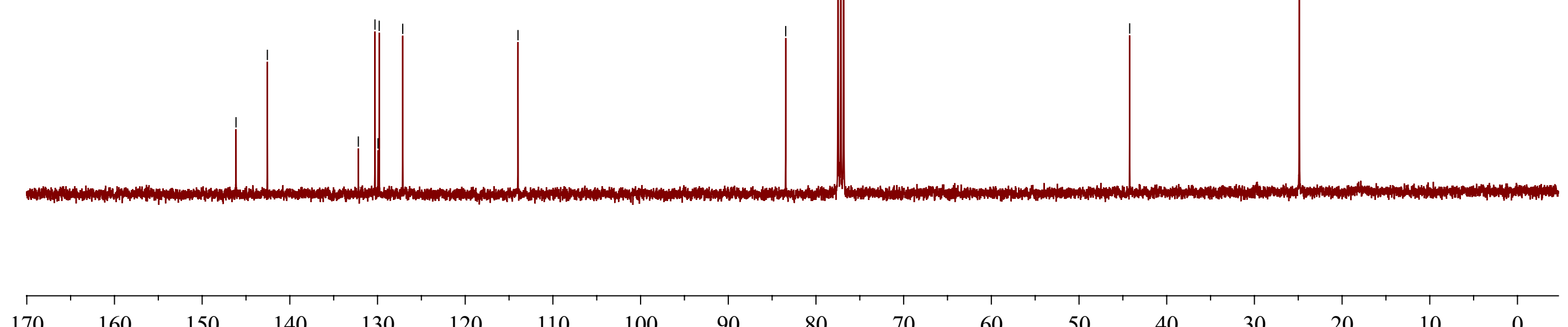
in $\mathrm{CDCl}_{3}, 400 \mathrm{MHz}$
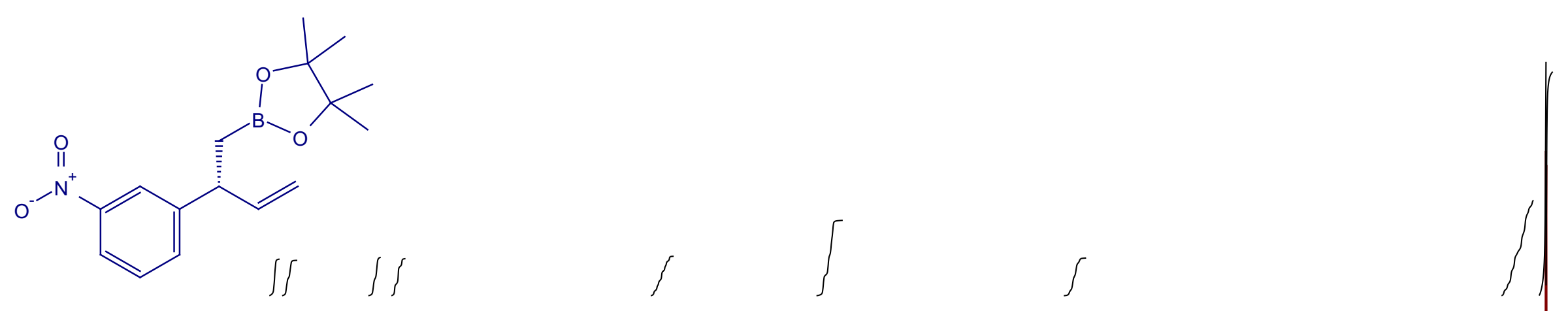

$12 k$

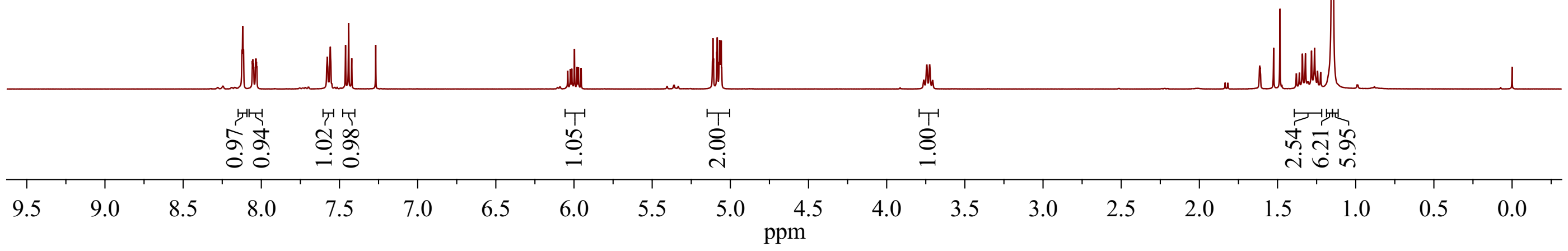




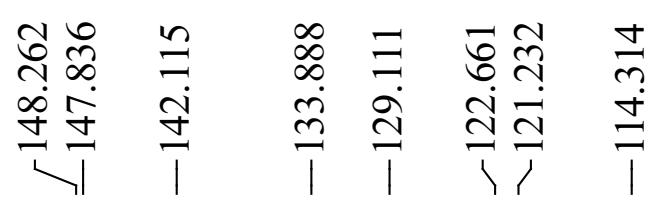

in $\mathrm{CDCl}_{3}, 101 \mathrm{MHz}$

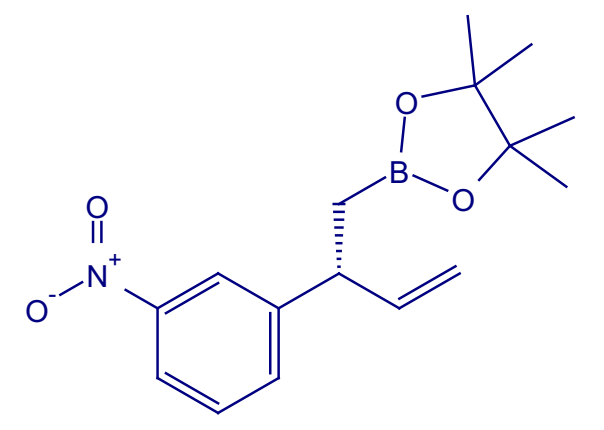

$12 k$
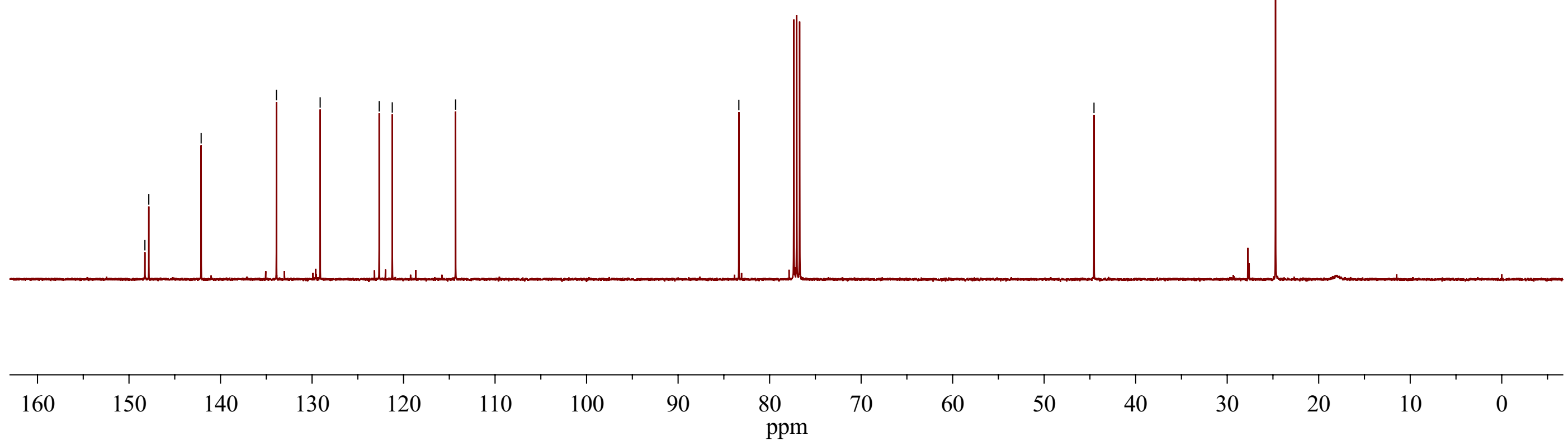

80
ppm

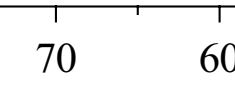

60

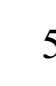

$50 \quad 40$

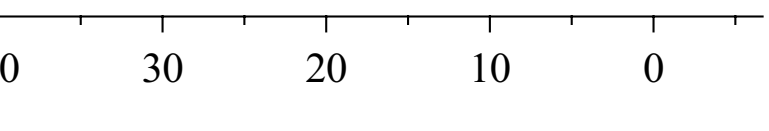


in $\mathrm{CDCl}_{3}, 400 \mathrm{MHz}$
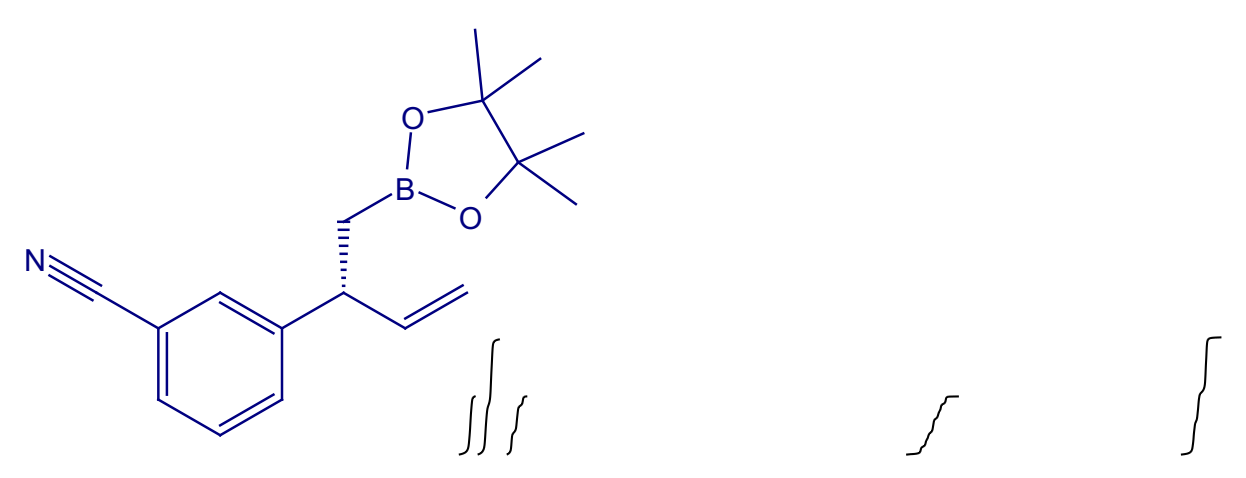

121

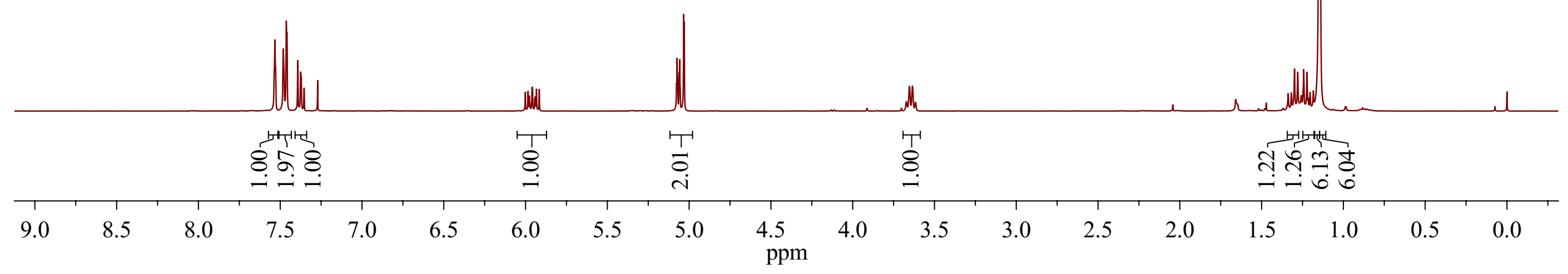




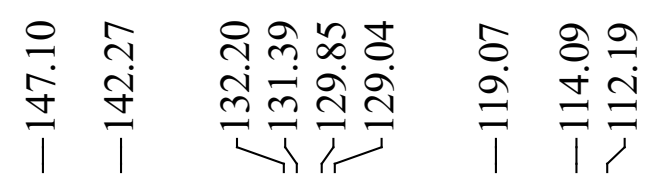

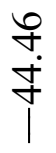

in $\mathrm{CDCl}_{3}, 101 \mathrm{MHz}$
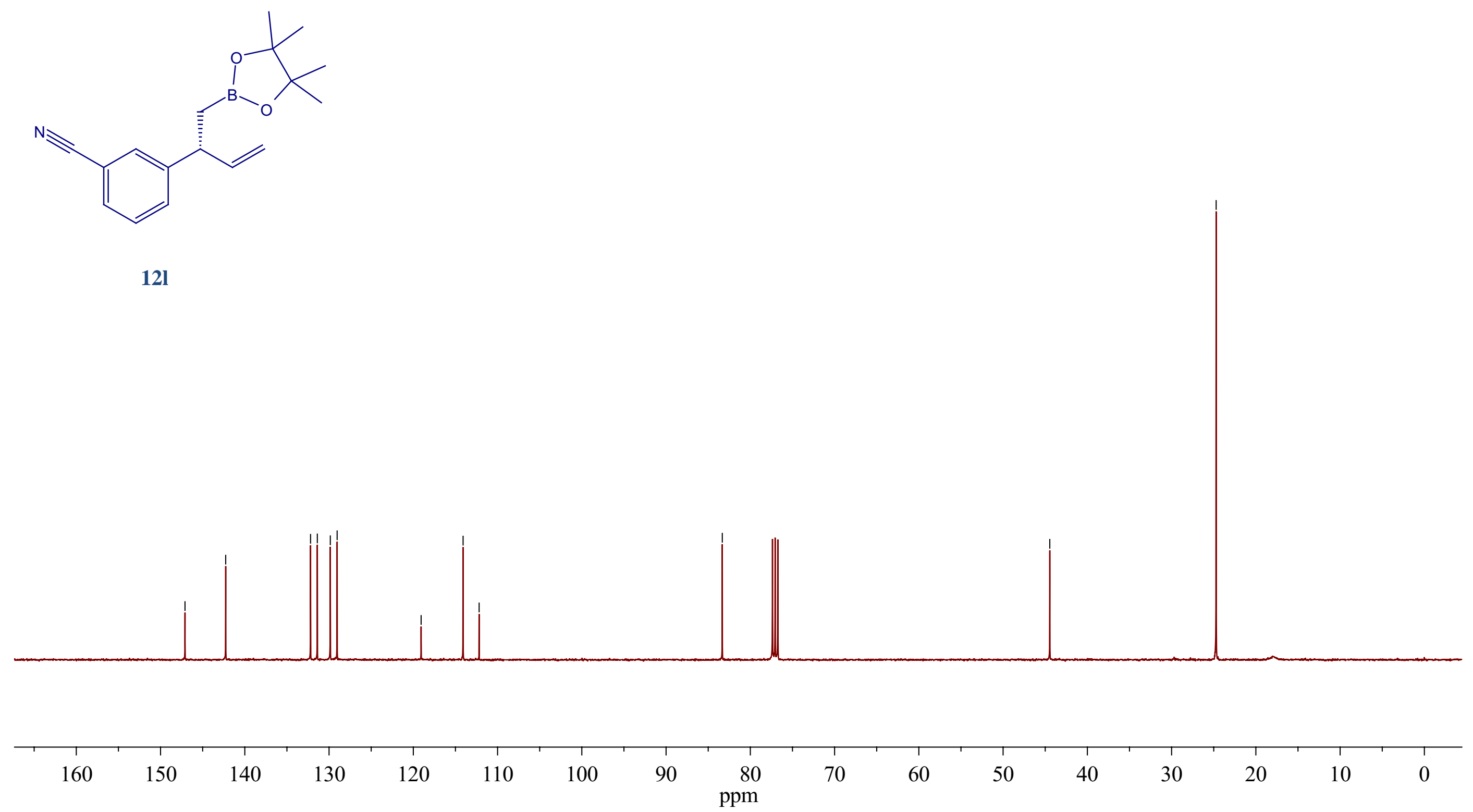


\section{in $\mathrm{CDCl}_{3}, 400 \mathrm{MHz}$}

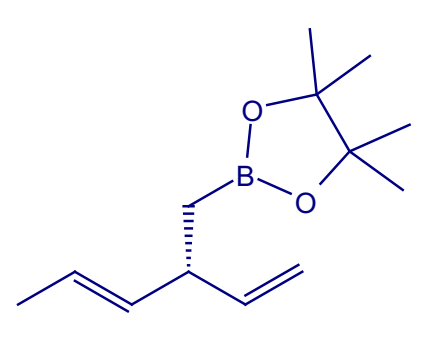

$12 \mathrm{~m}$
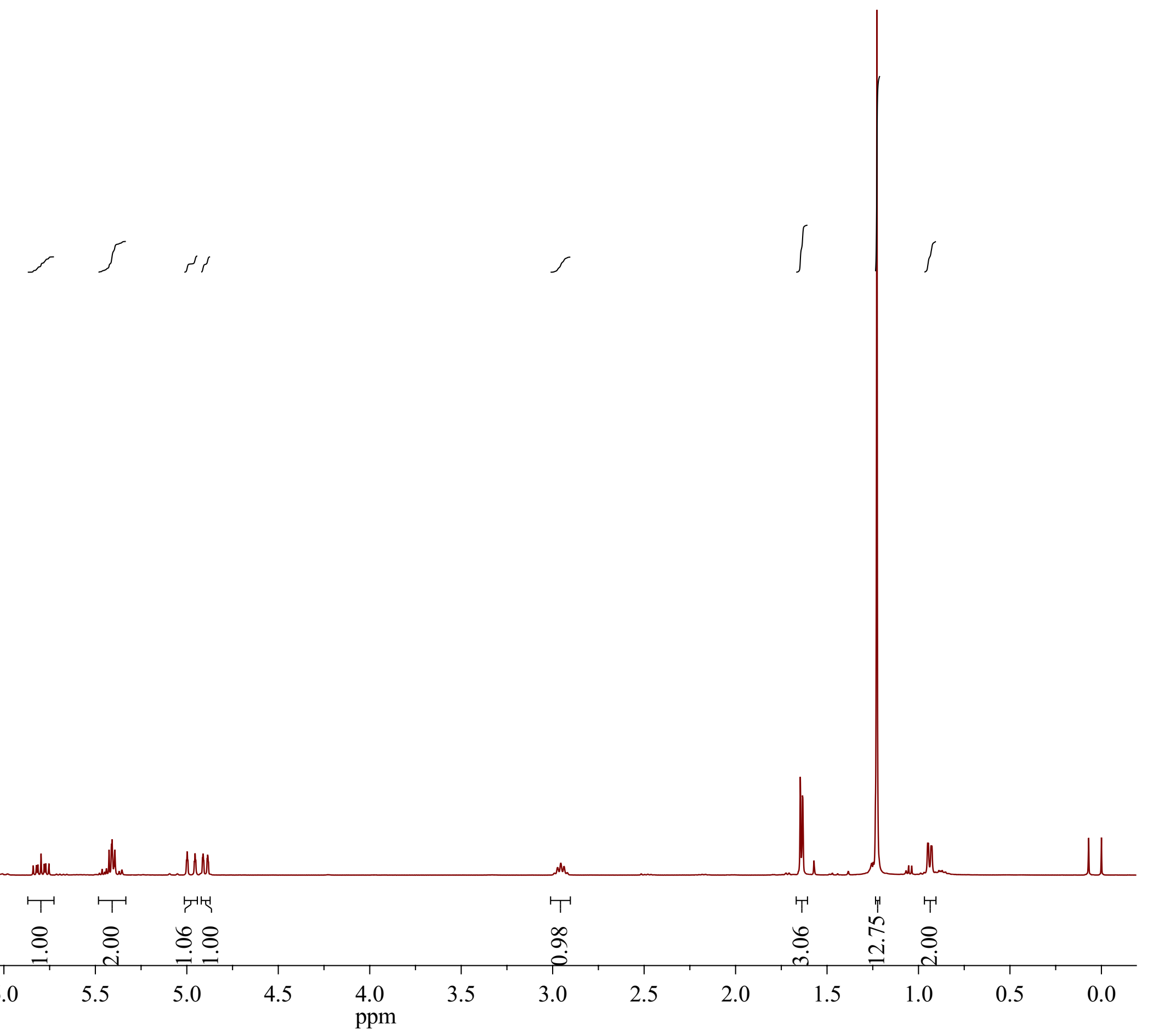


\begin{tabular}{|c|c|c|c|c|c|}
\hline $\begin{array}{l}\stackrel{q}{d} \\
\stackrel{n}{1}\end{array}$ & $\begin{array}{l}\infty \\
\infty \\
\\
\end{array}$ & 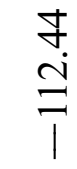 & 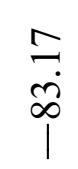 & 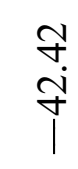 & 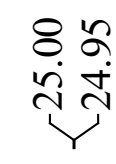 \\
\hline
\end{tabular}

in $\mathrm{CDCl}_{3}, 101 \mathrm{MHz}$

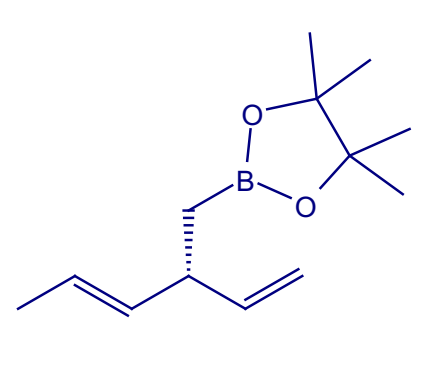

$12 m$

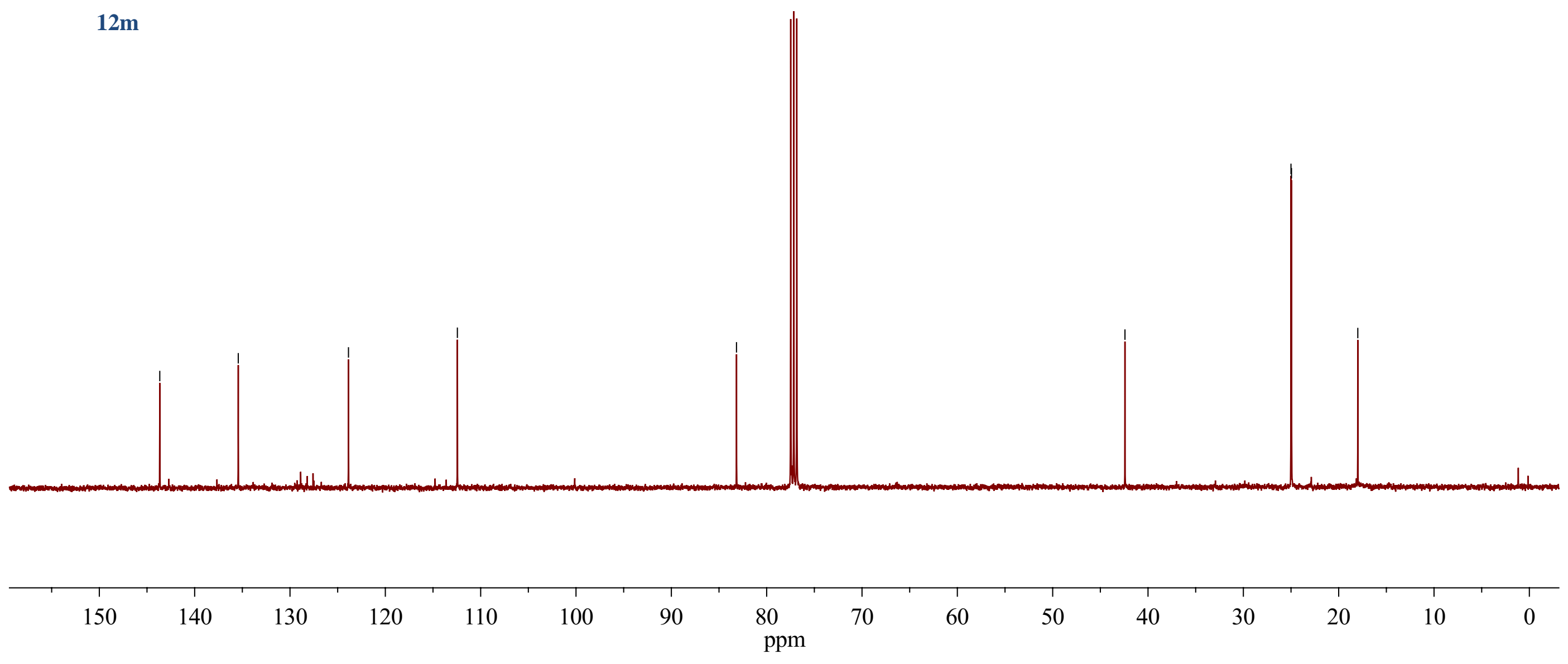




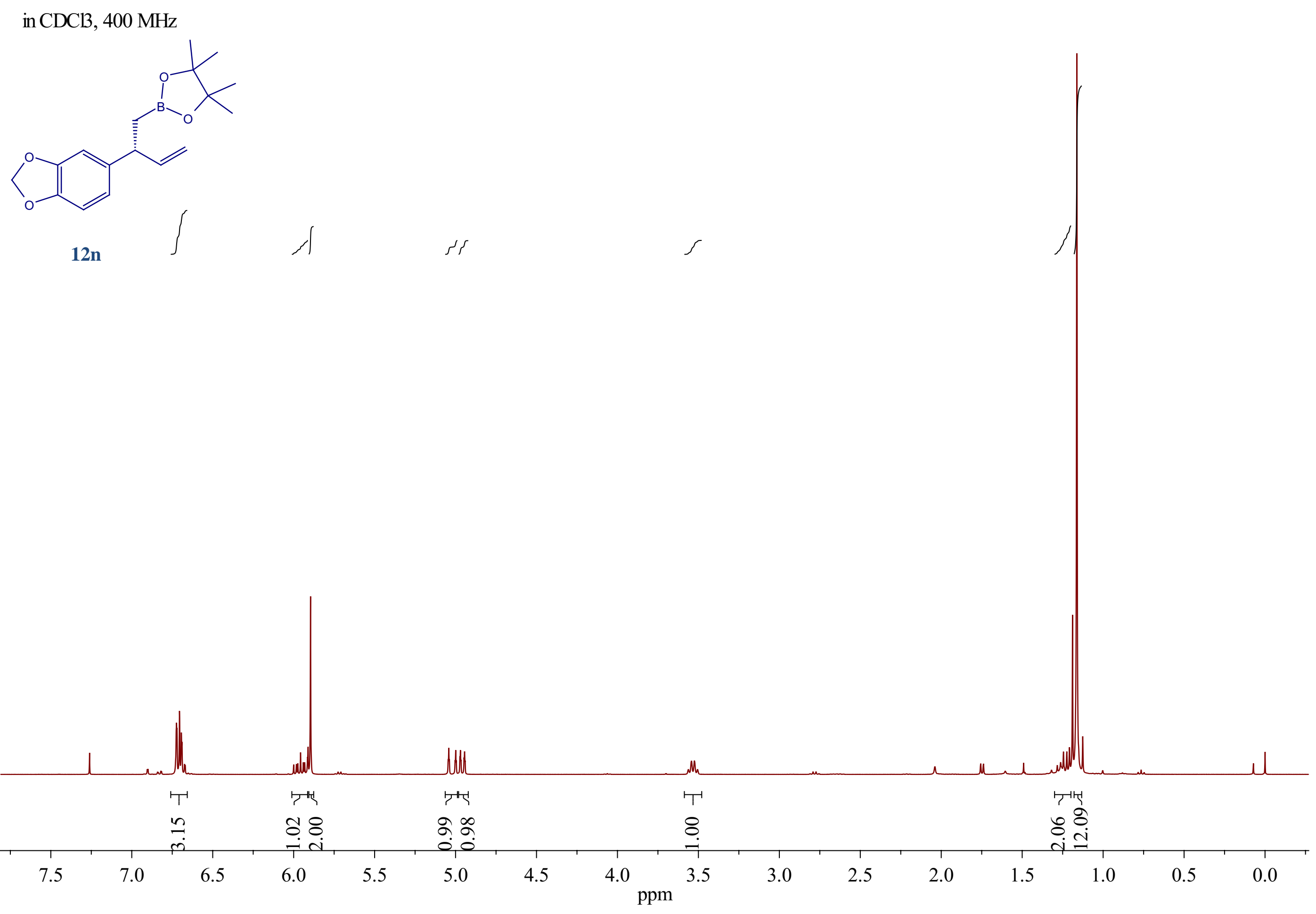


ஸิ?

守守离

寺吉

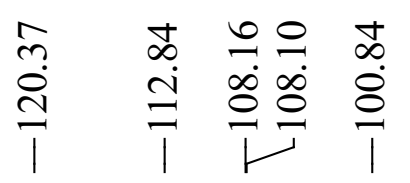

ñ

$8 \infty$
$\stackrel{8}{+}$
$\stackrel{+}{j}$

in CDCB, $101 \mathrm{MHz}$

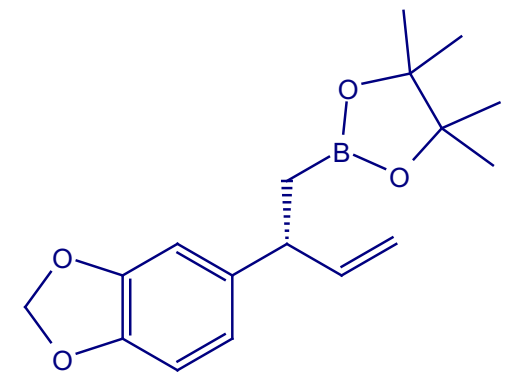

12n

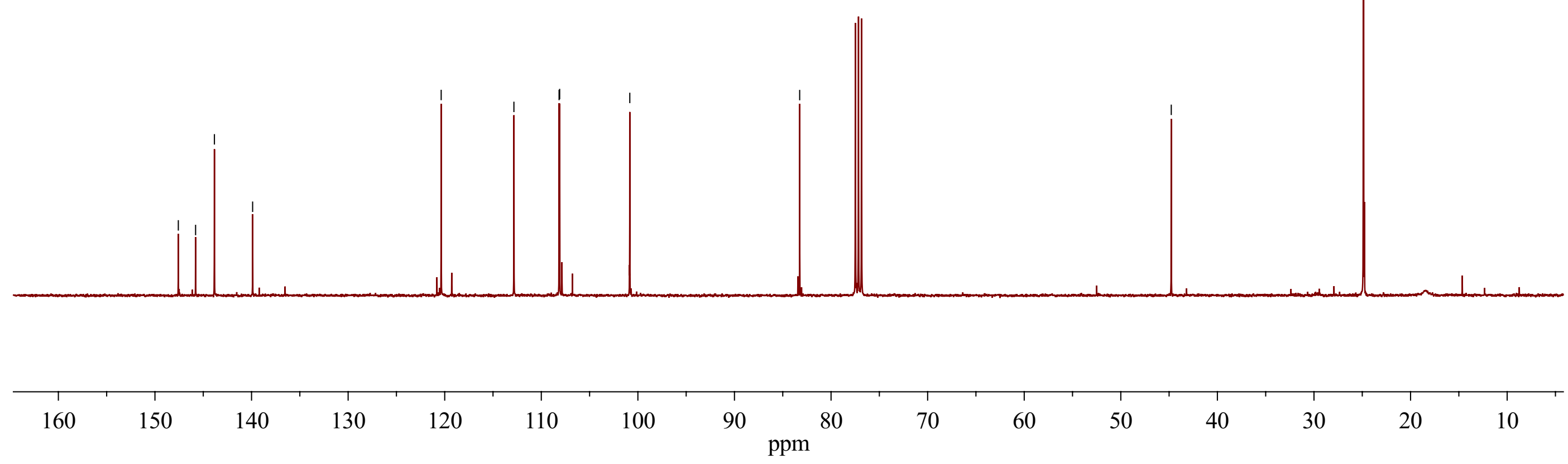




\section{in $\mathrm{CDCl}_{3}, 400 \mathrm{MHz}$}
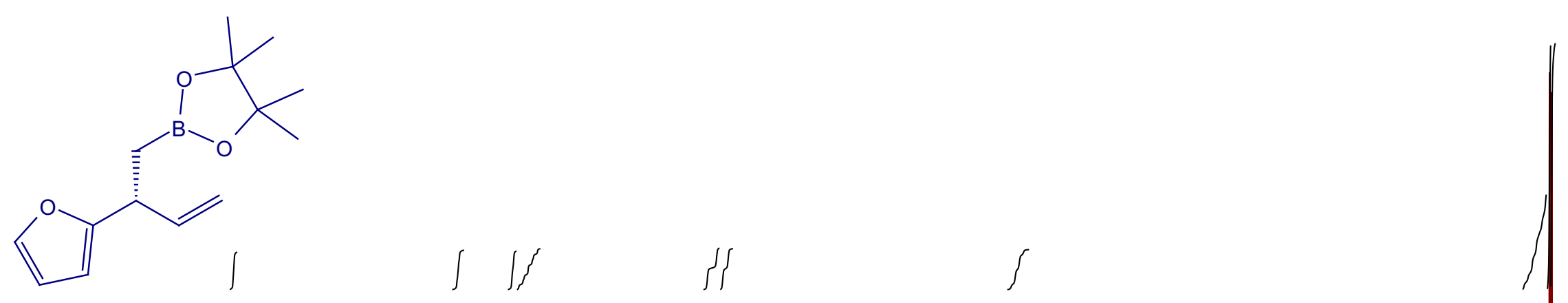

120

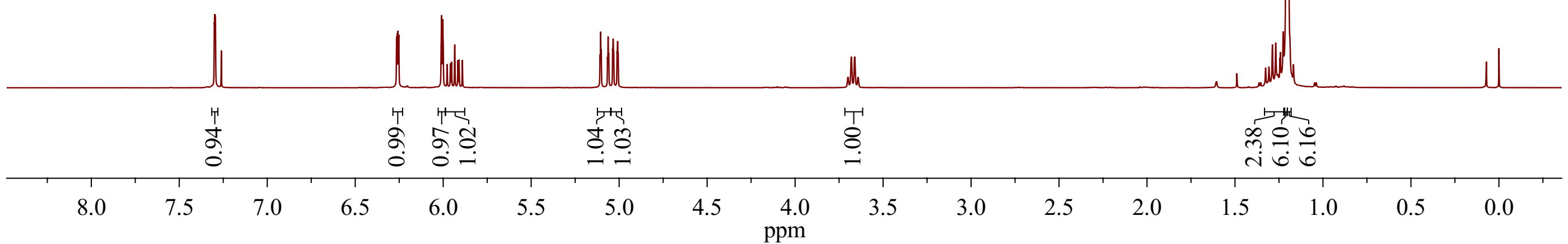




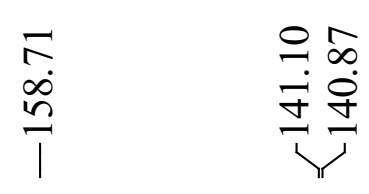

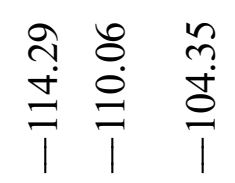

$\underset{n}{m}$

in $\mathrm{CDCl}_{3}, 101 \mathrm{MHz}$

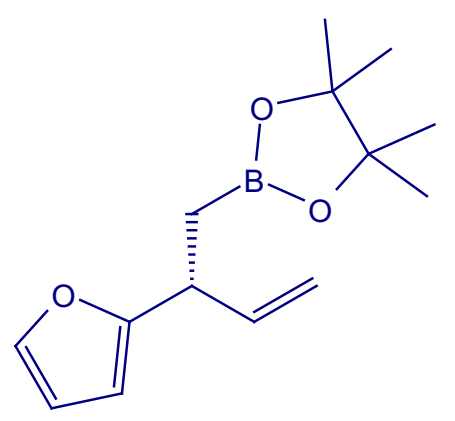

120

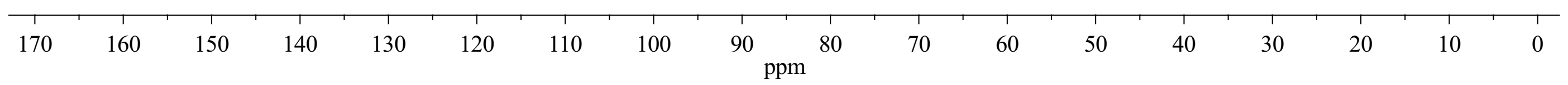


in $\mathrm{CDCl}, 400 \mathrm{MHz}$
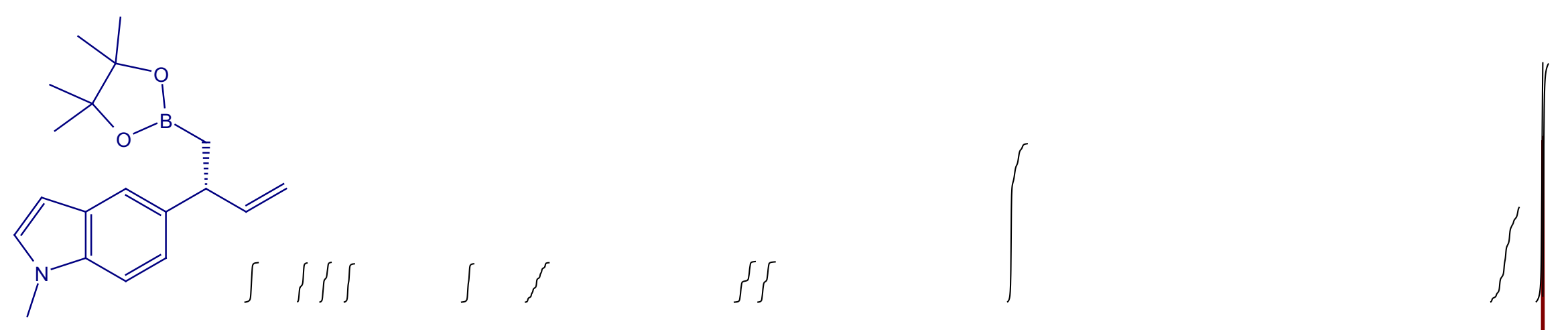

$12 p$

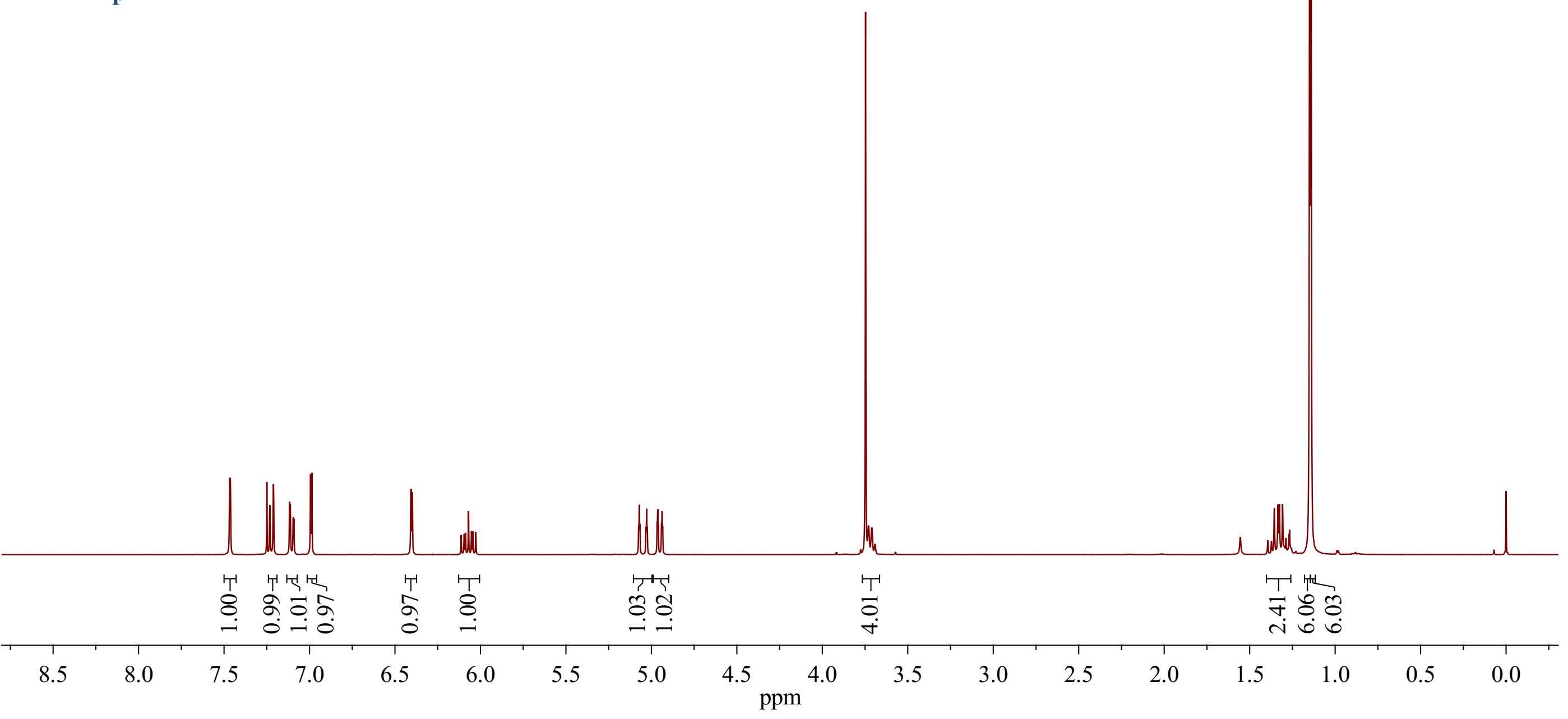


in CDCB, $101 \mathrm{MHz}$

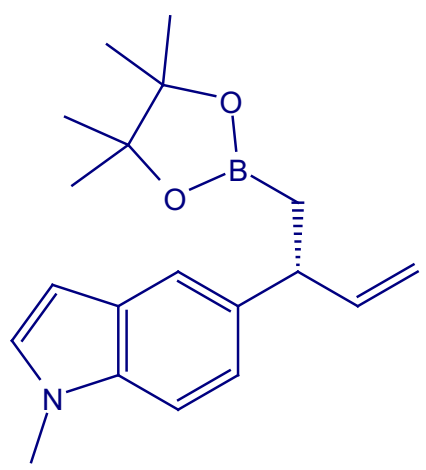

$12 p$

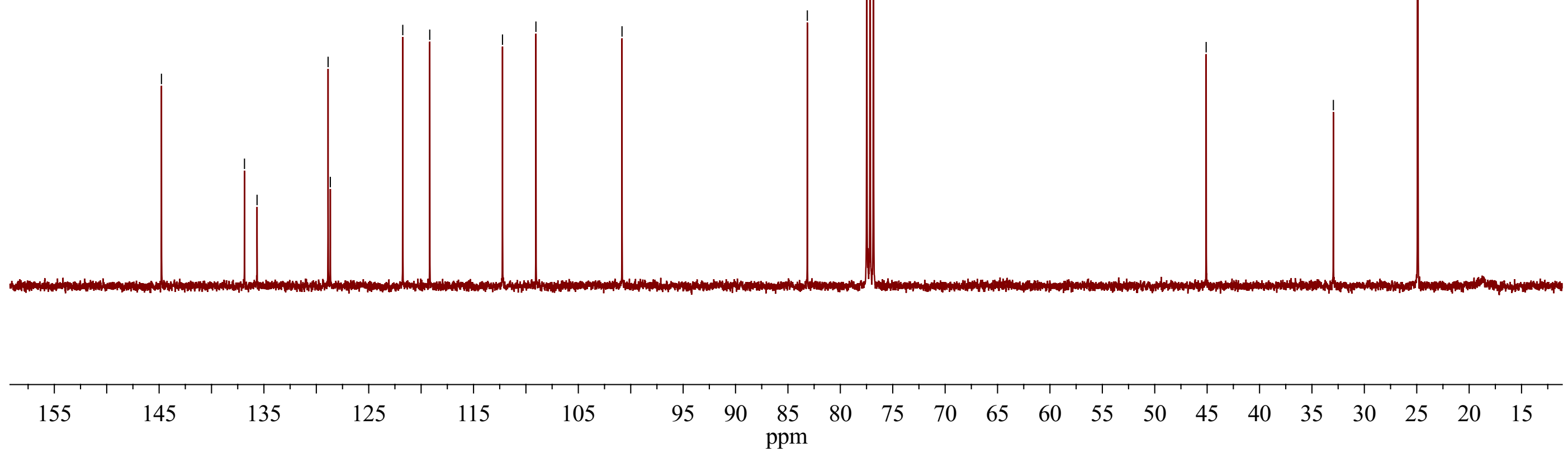


in $\mathrm{CDCB}, 400 \mathrm{MHz}$
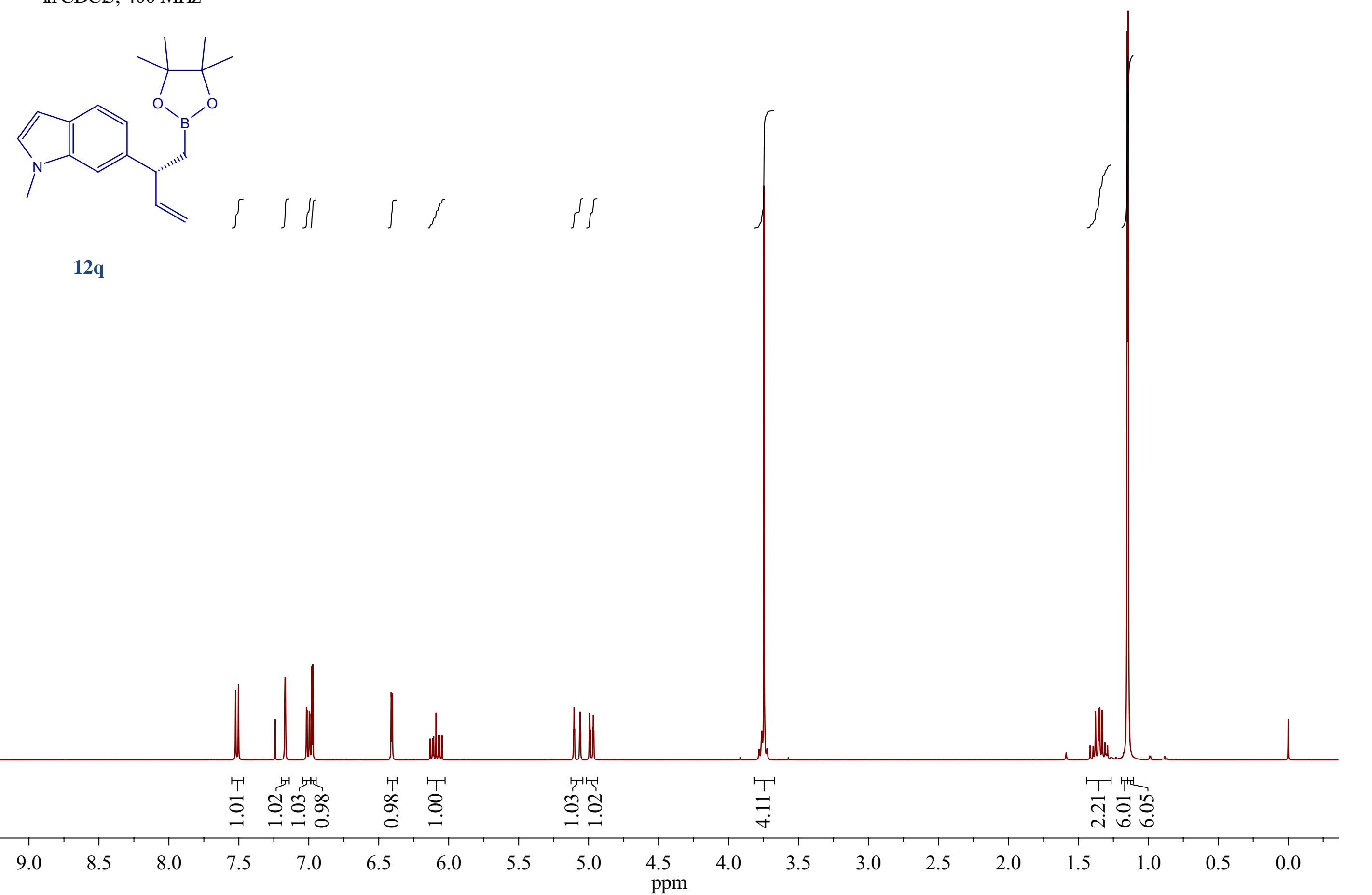


\begin{tabular}{|c|c|c|c|c|c|c|c|}
\hline $\begin{array}{l}30 \\
\dot{0} \\
\dot{m} \\
1\end{array}$ & 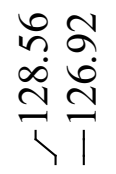 & 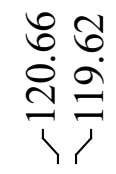 & 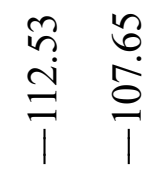 & $\begin{array}{l}\stackrel{8}{0} \\
\stackrel{8}{1}\end{array}$ & $\frac{1}{\infty}$ & 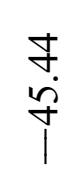 & $\begin{array}{l}\infty \\
\infty \\
i \\
i\end{array}$ \\
\hline
\end{tabular}

in $\mathrm{CDCB}, 101 \mathrm{MHz}$

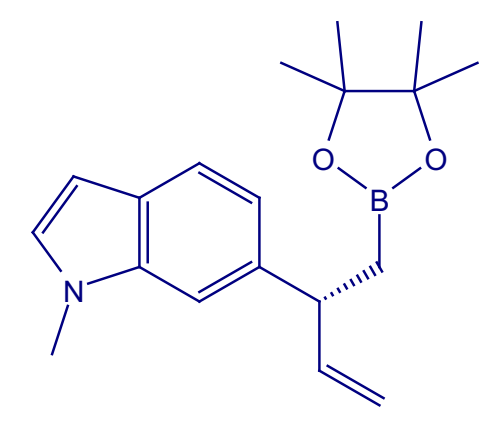

$12 q$

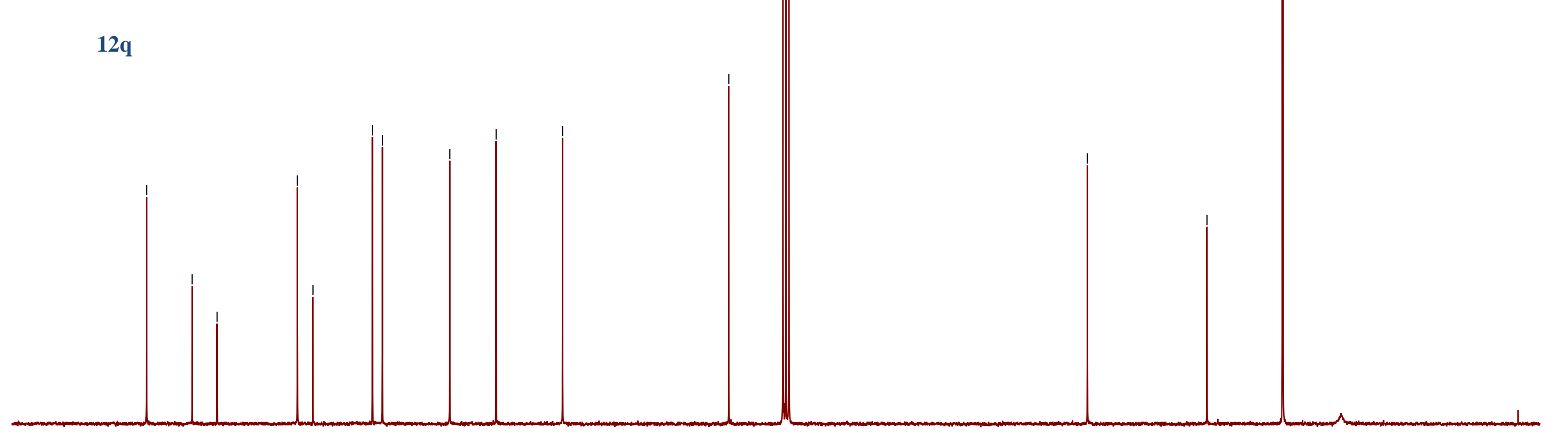


in $\mathrm{CDCB}$, $400 \mathrm{MHz}$
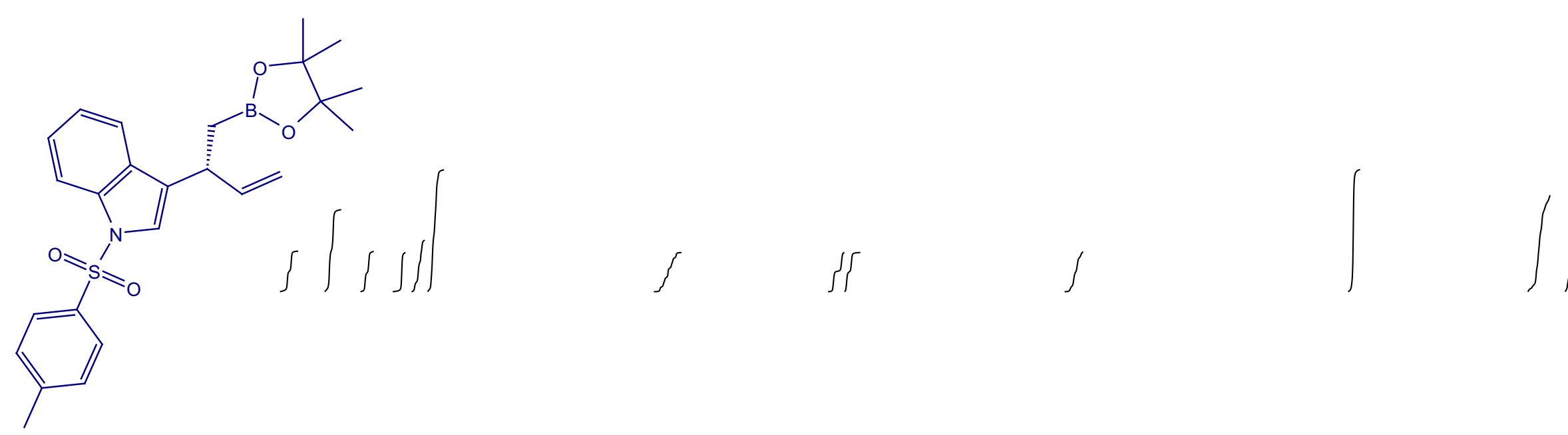

$12 r$

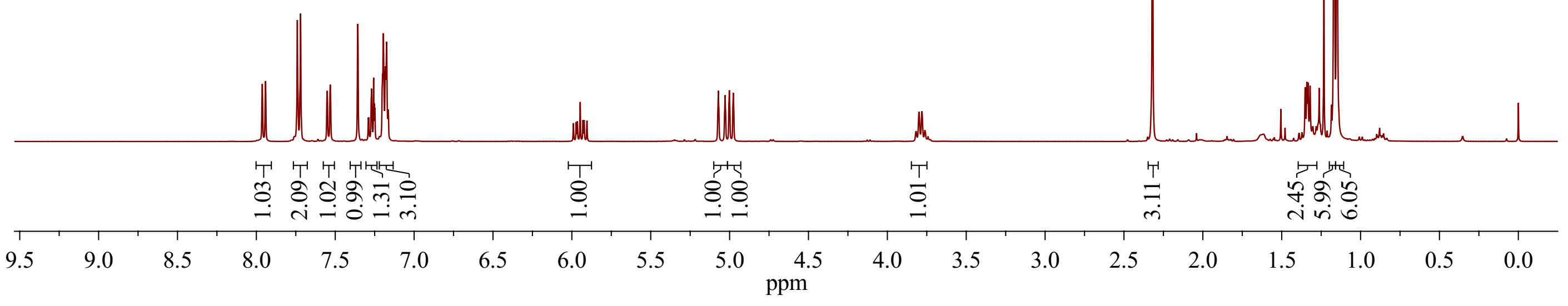




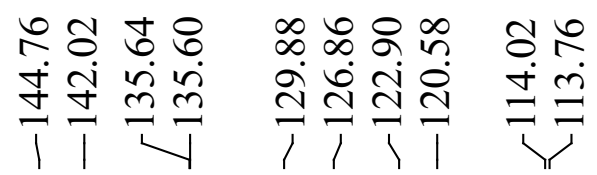

in $\mathrm{CDCl}, 101 \mathrm{MHz}$
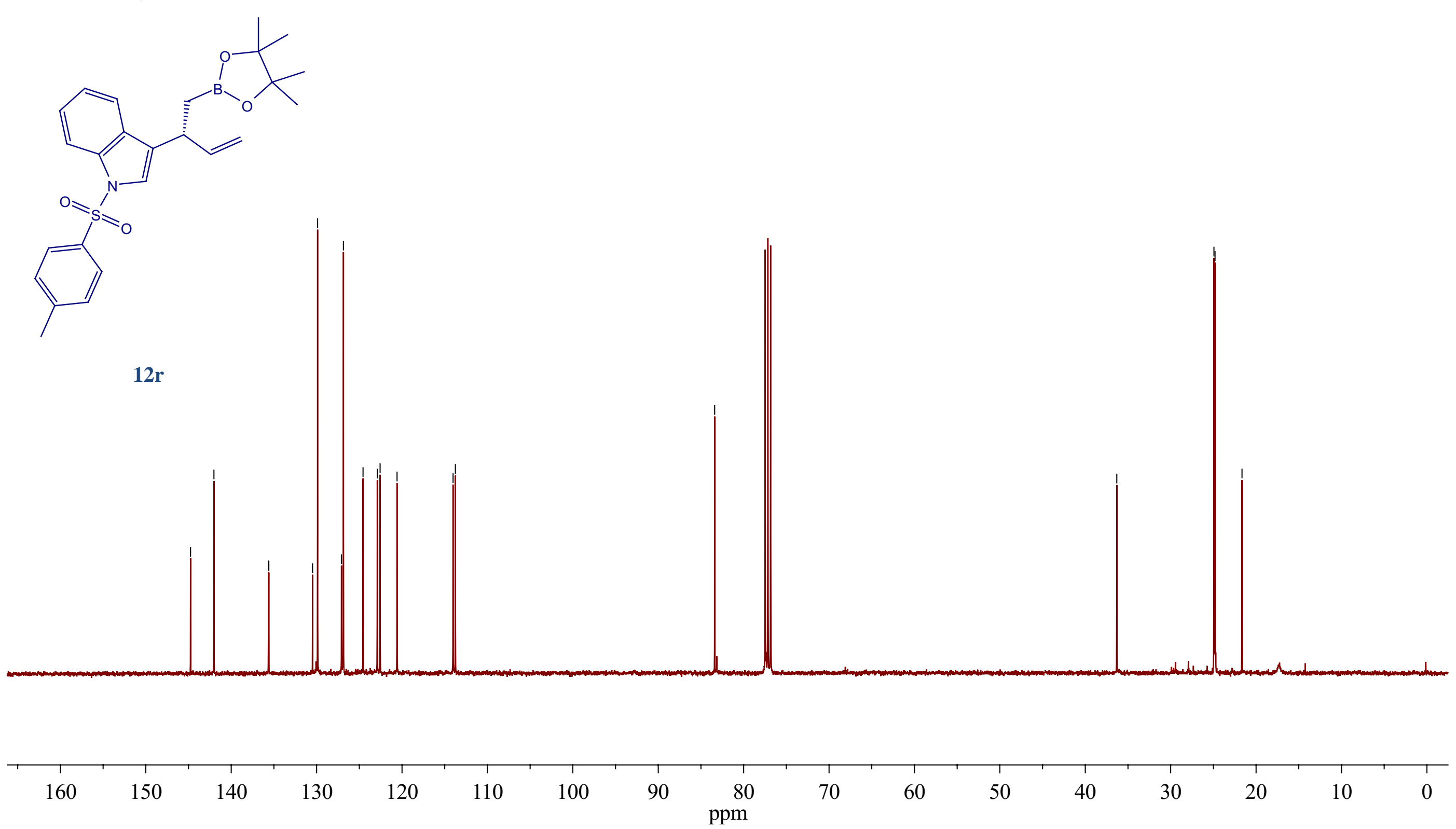
in CDCB, $400 \mathrm{MHz}$
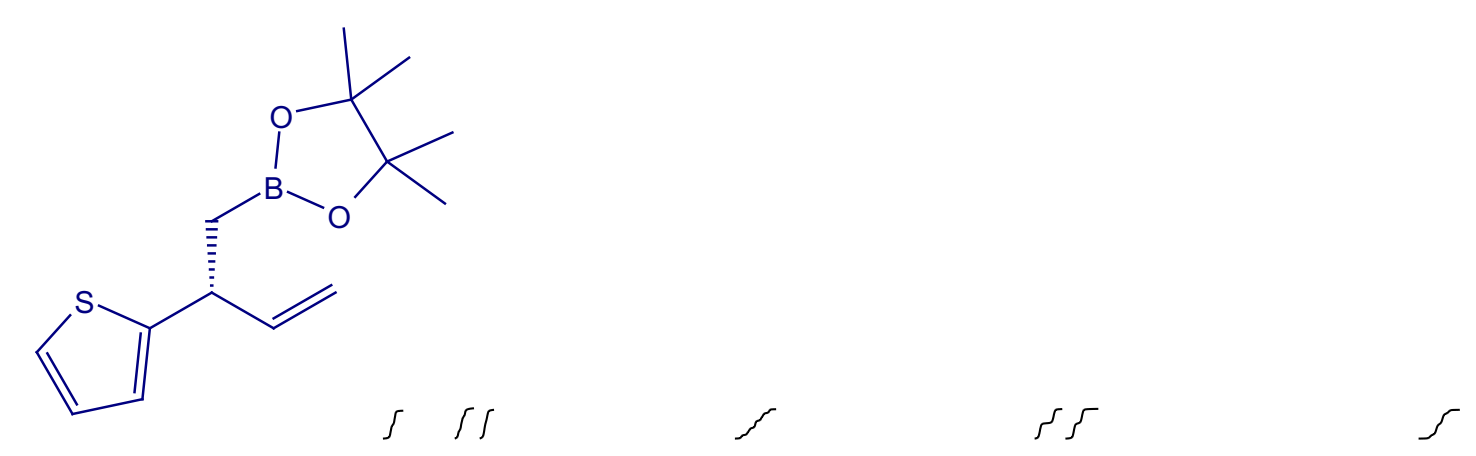

$12 \mathrm{~s}$

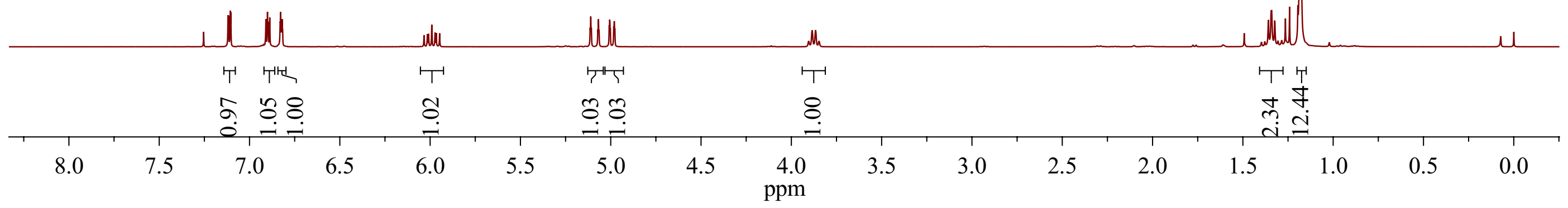




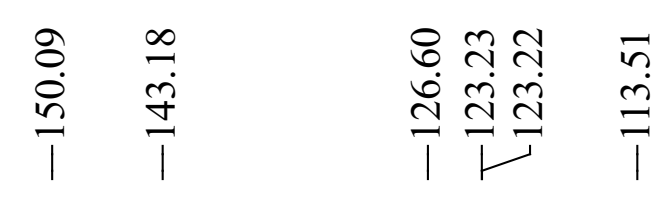

in $\mathrm{CDCl}$, $101 \mathrm{MHz}$

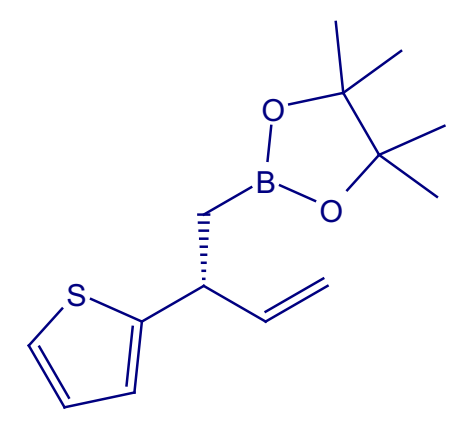

12s

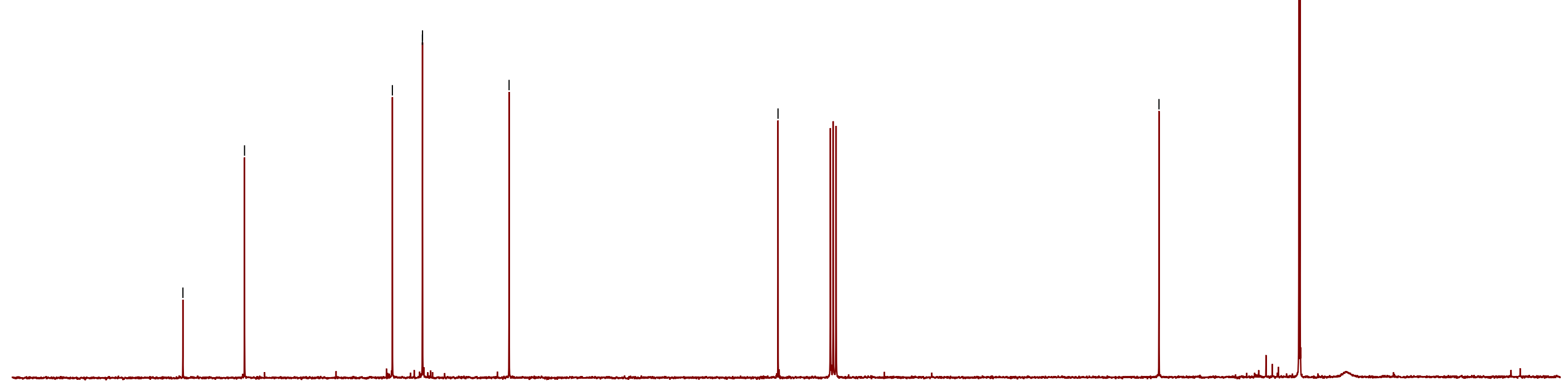


in $\mathrm{CDCB}, 400 \mathrm{MHz}$
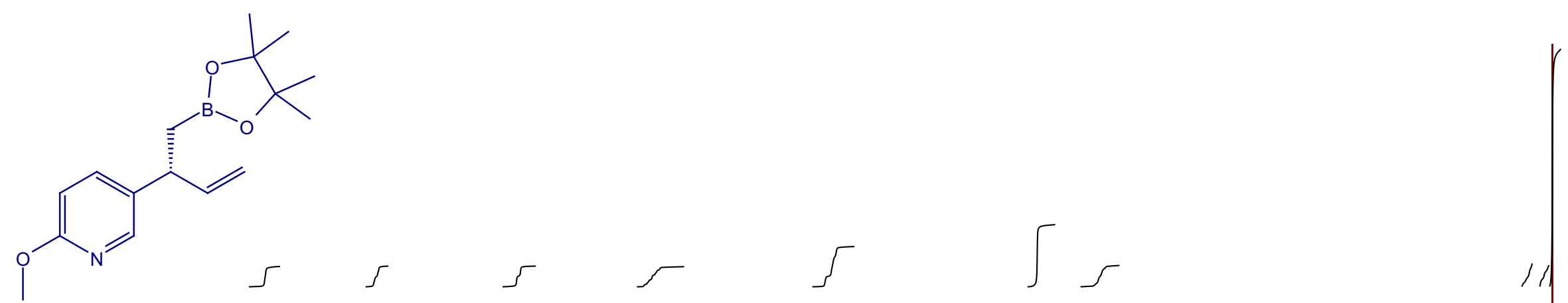

$12 t$

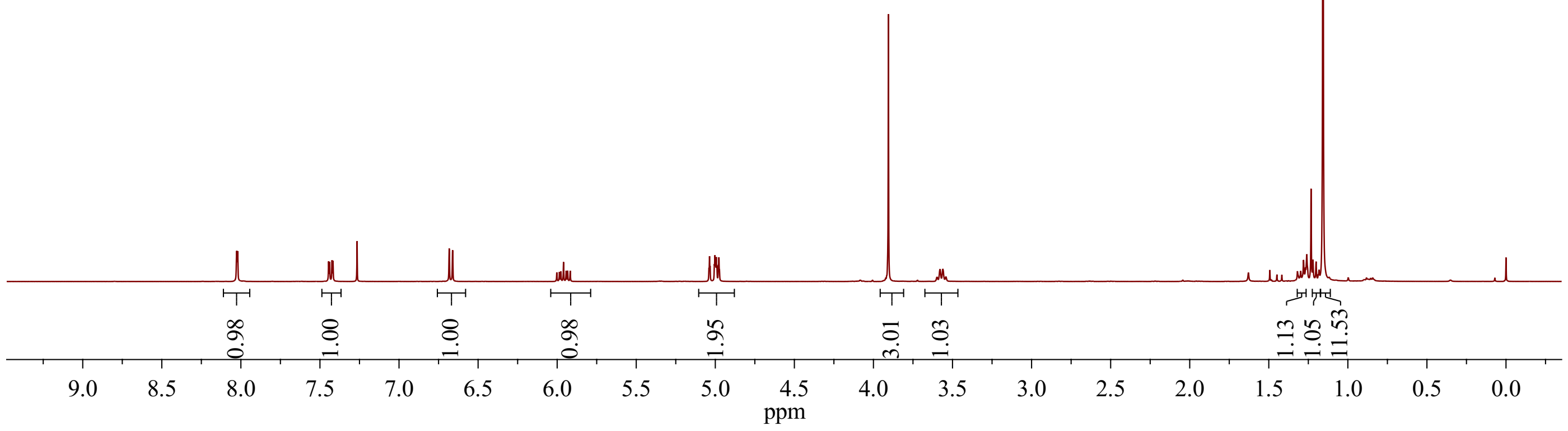




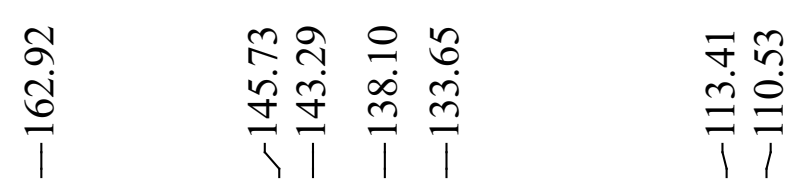

in $\mathrm{CDCB}, 101 \mathrm{MHz}$

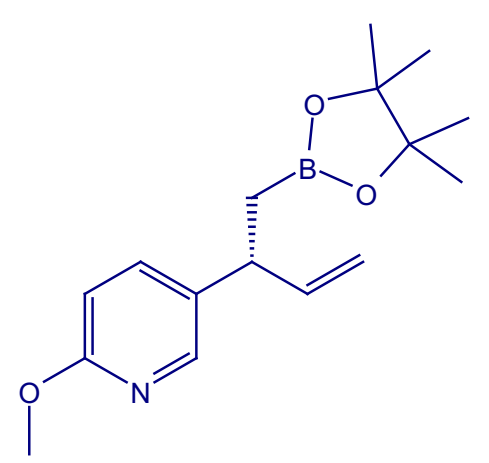

$12 t$
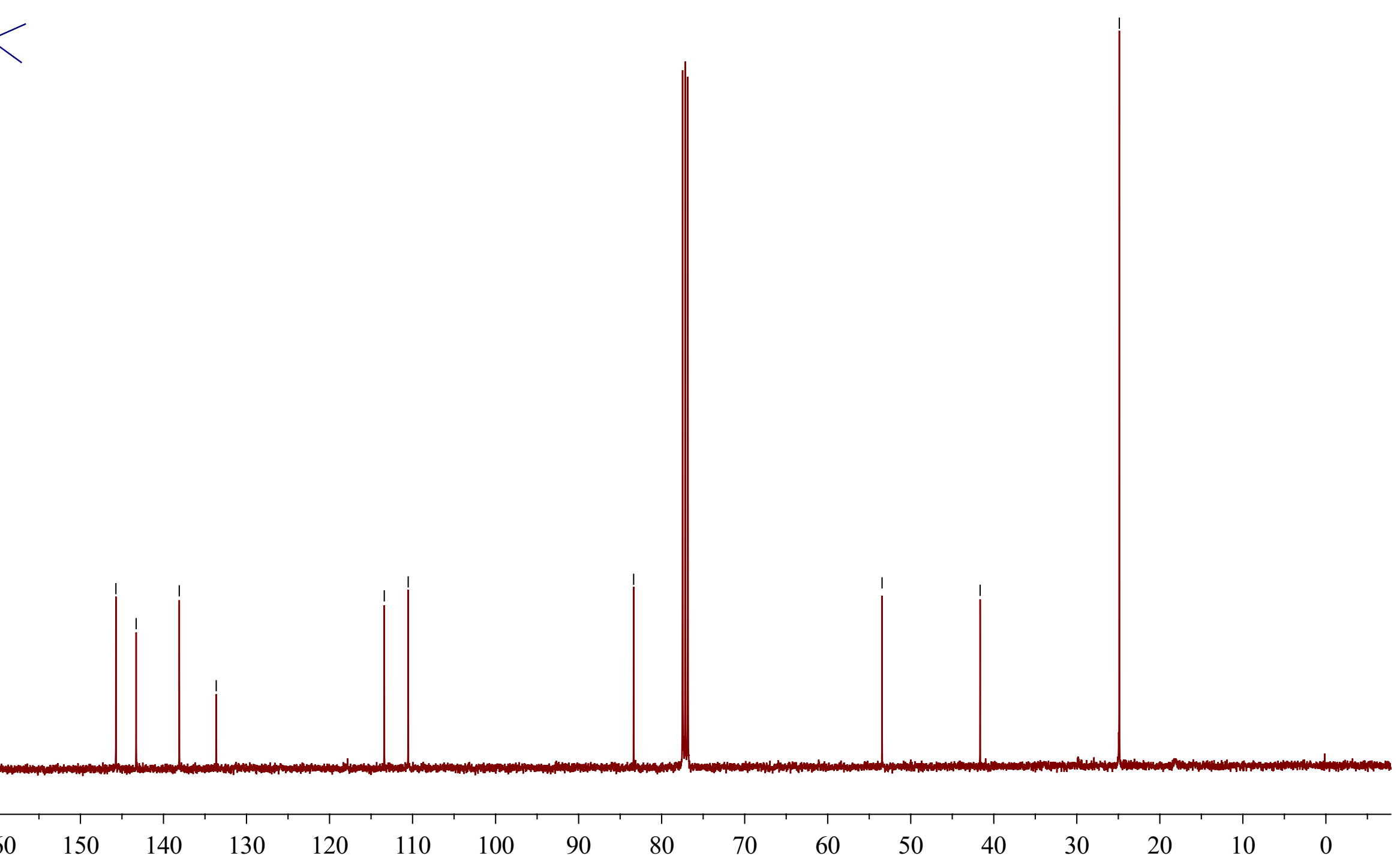

150

140

130 
in $\mathrm{CDCl}_{3}, 400 \mathrm{MHz}$
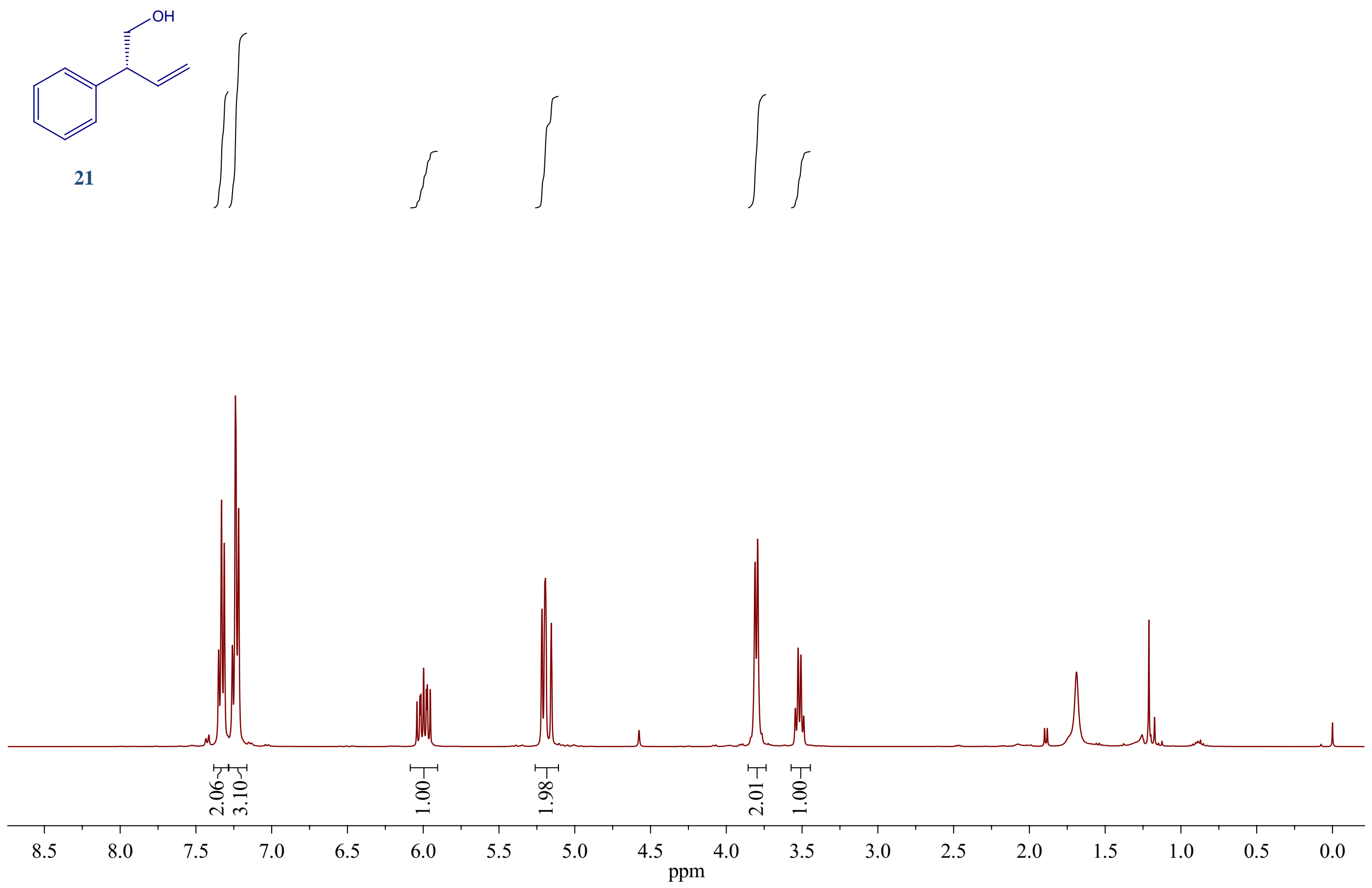


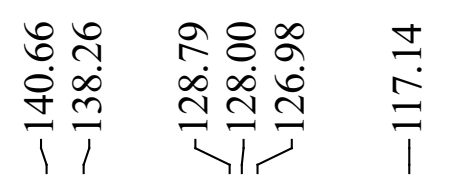

$\begin{array}{ll}\text { l } & \text { in } \\ 0 & \text { in } \\ 0 & \text { in }\end{array}$

in $\mathrm{CDCl}_{3}, 101 \mathrm{MHz}$

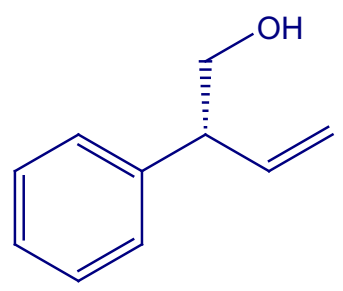

21

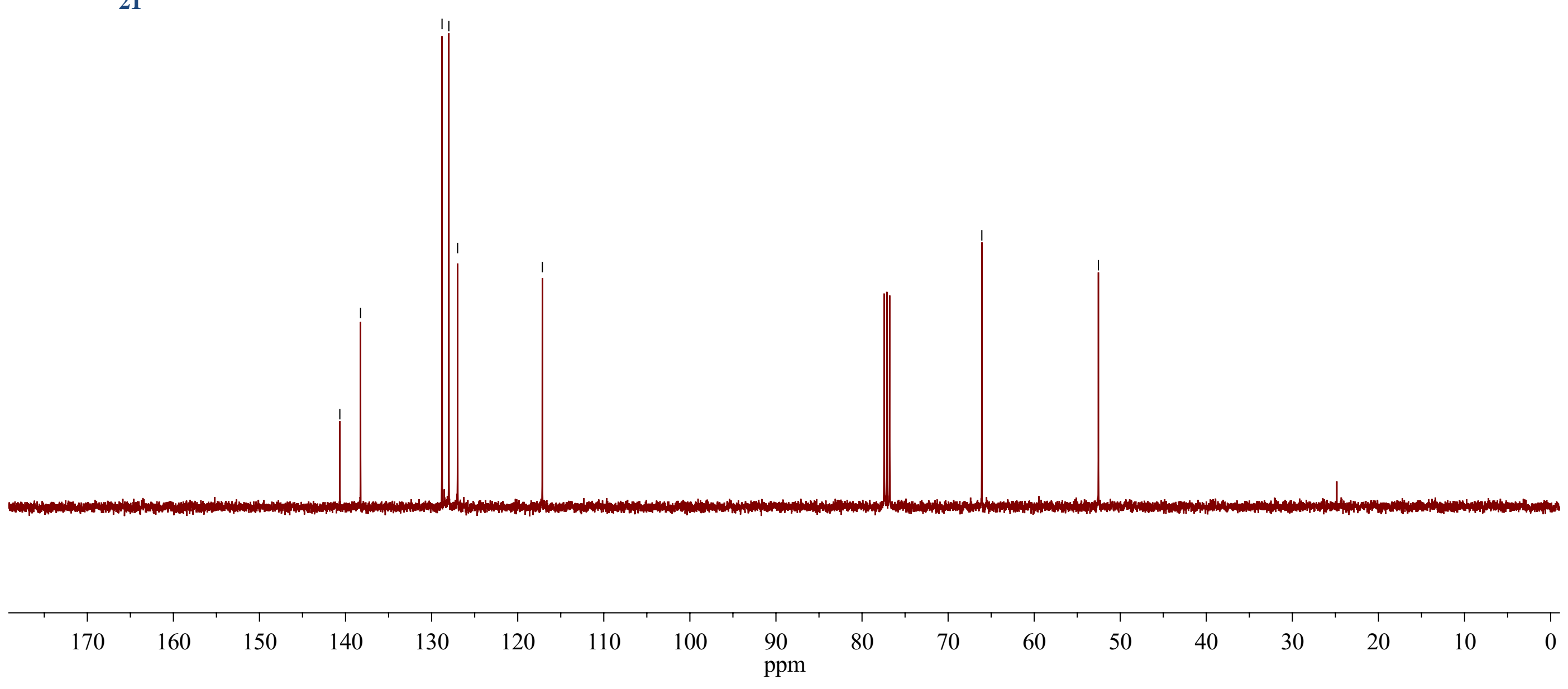


in $\mathrm{CDCl}_{3}, 400 \mathrm{MHz}$
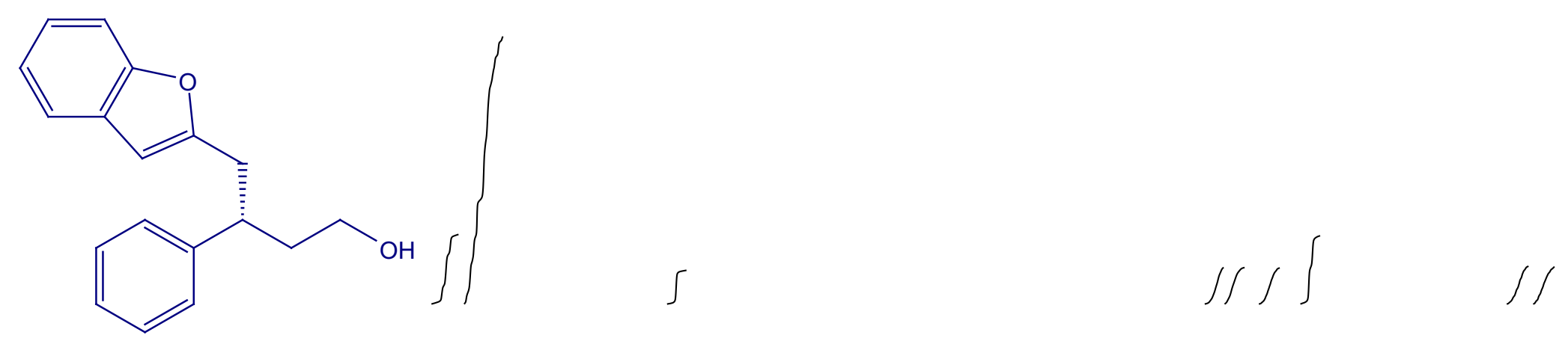

23

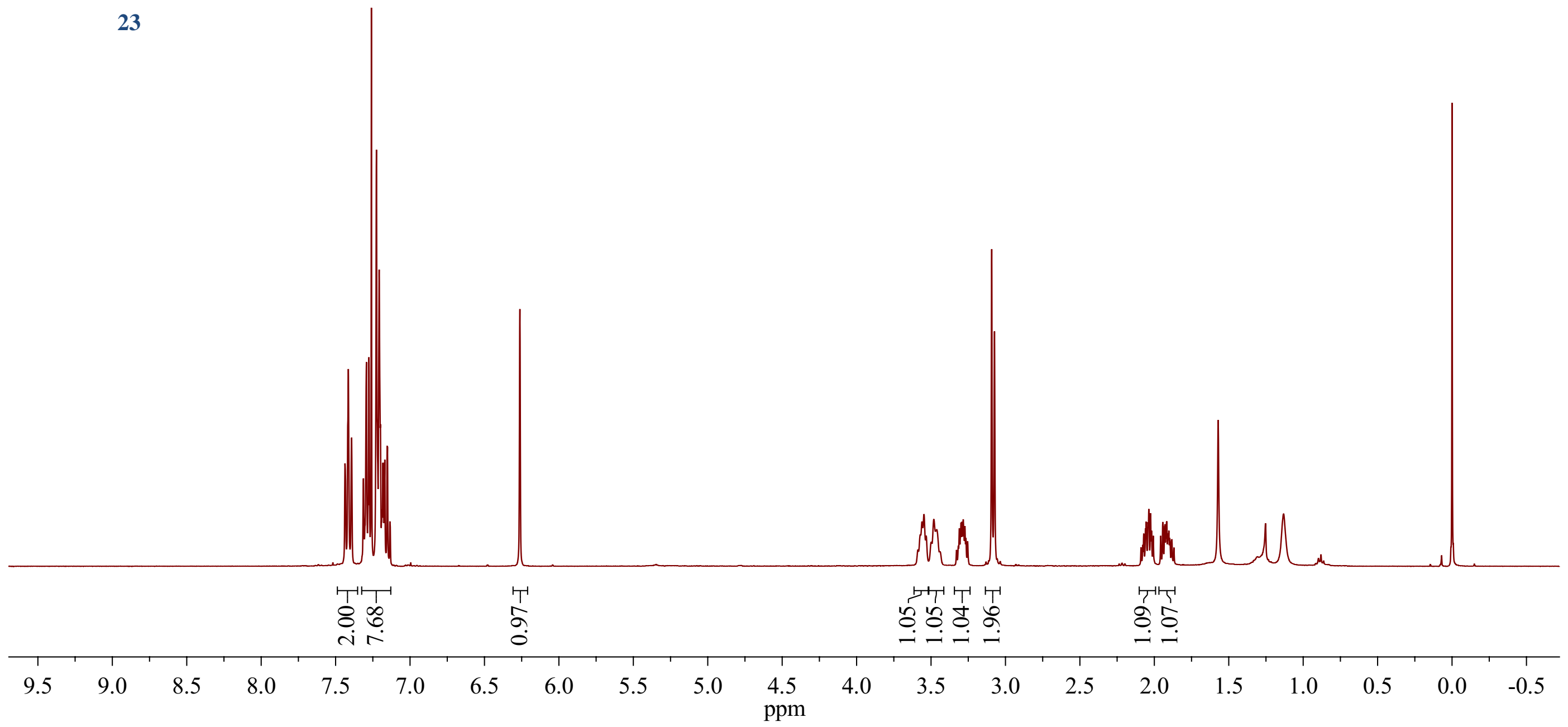




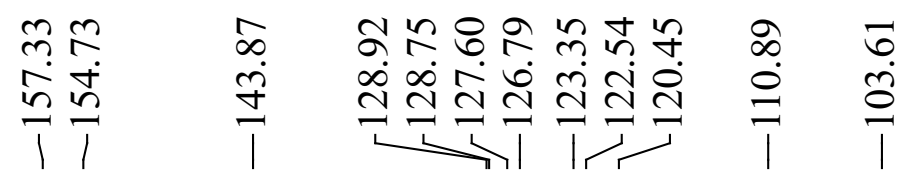

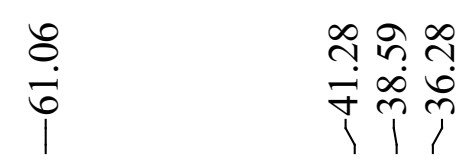

in $\mathrm{CDCl}_{3}, 101 \mathrm{MHz}$

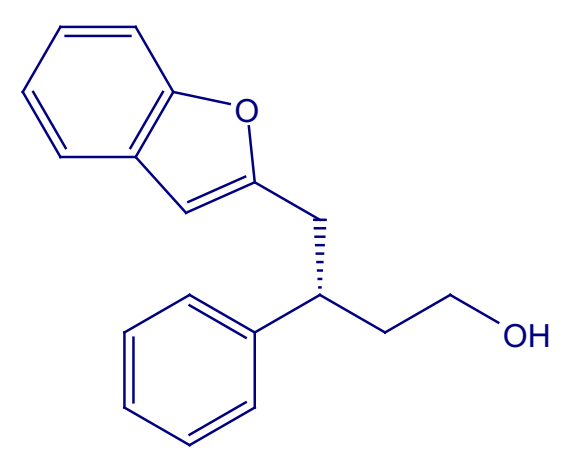

23

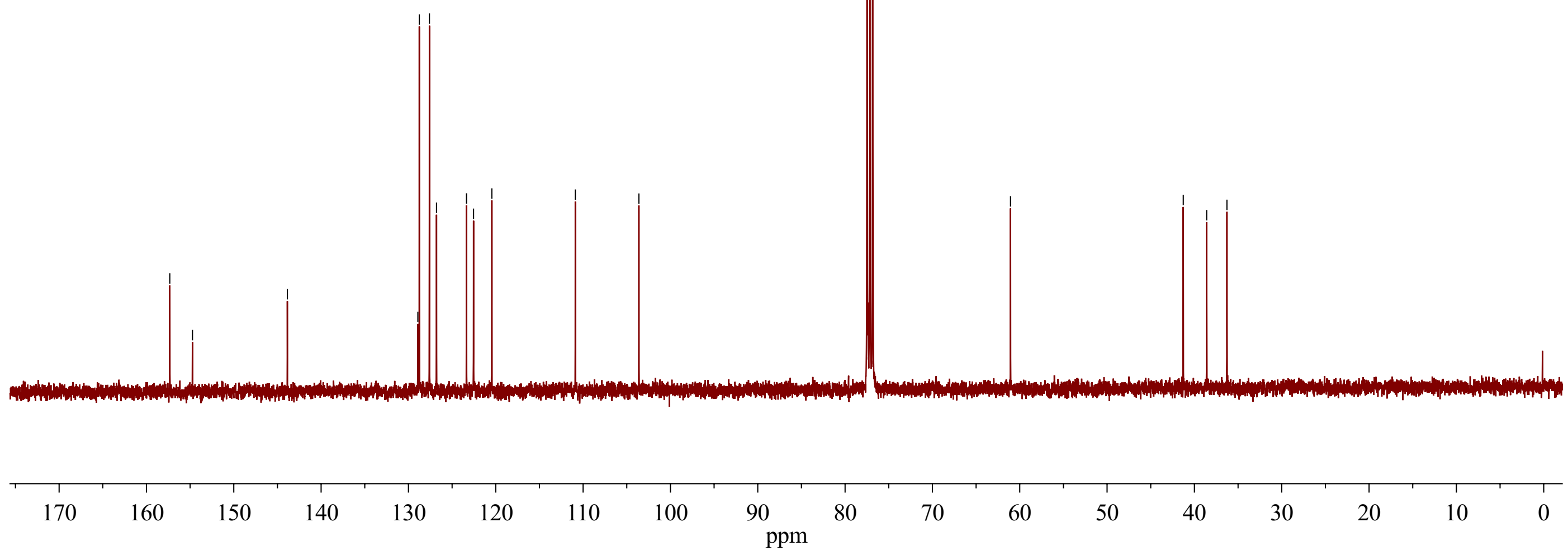


in $\mathrm{CDCl}_{3}, 400 \mathrm{MHz}$
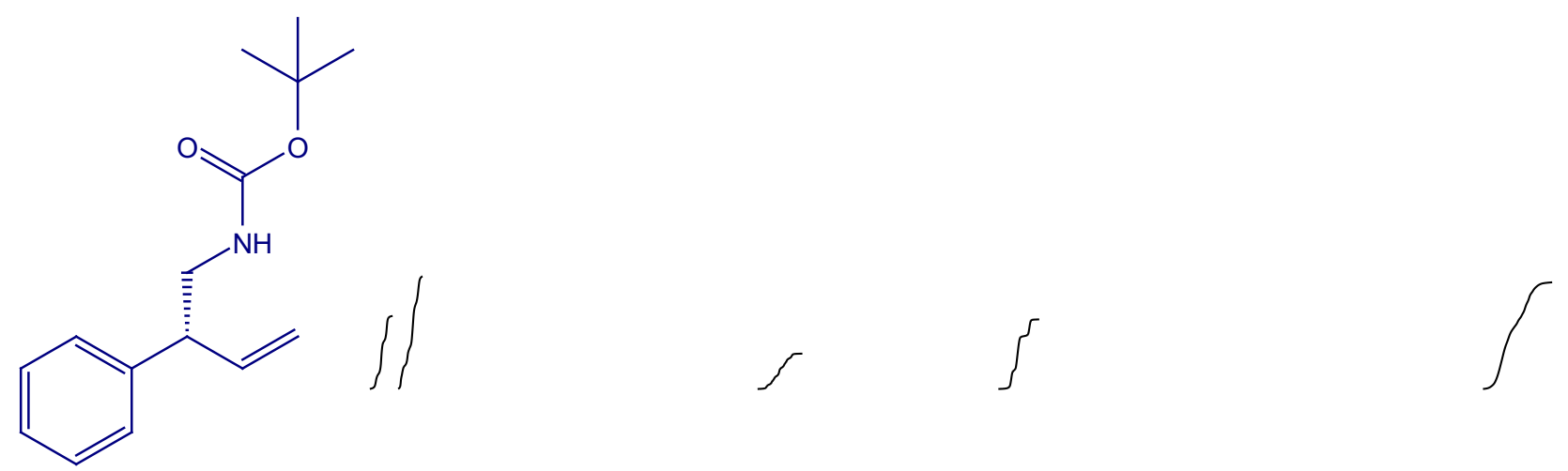

24

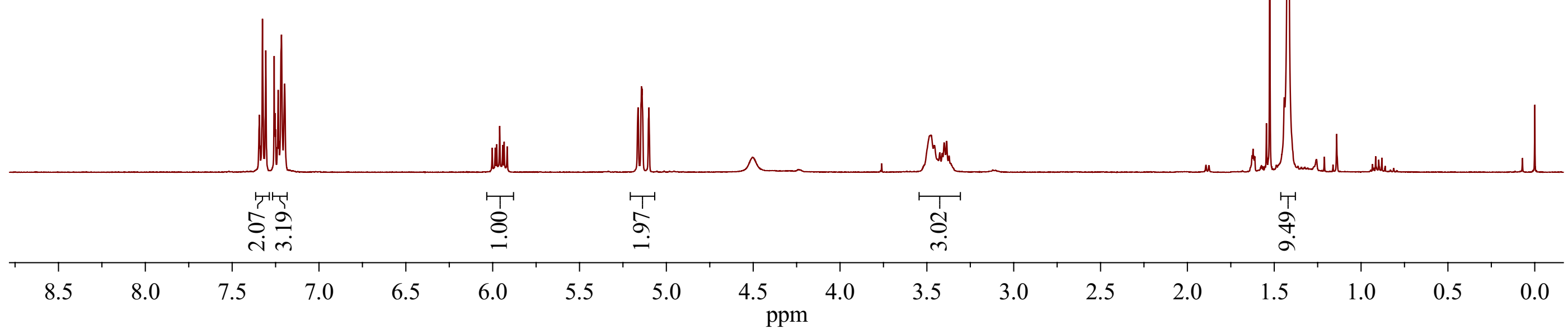




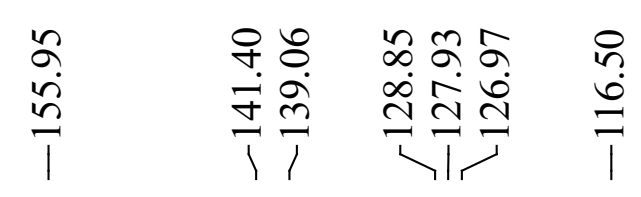

in $\mathrm{CDCl}_{3}, 101 \mathrm{MHz}$

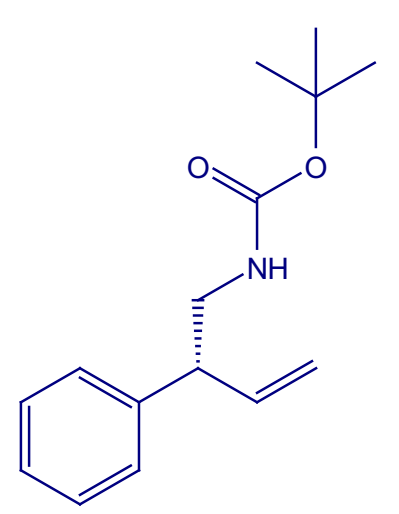

24
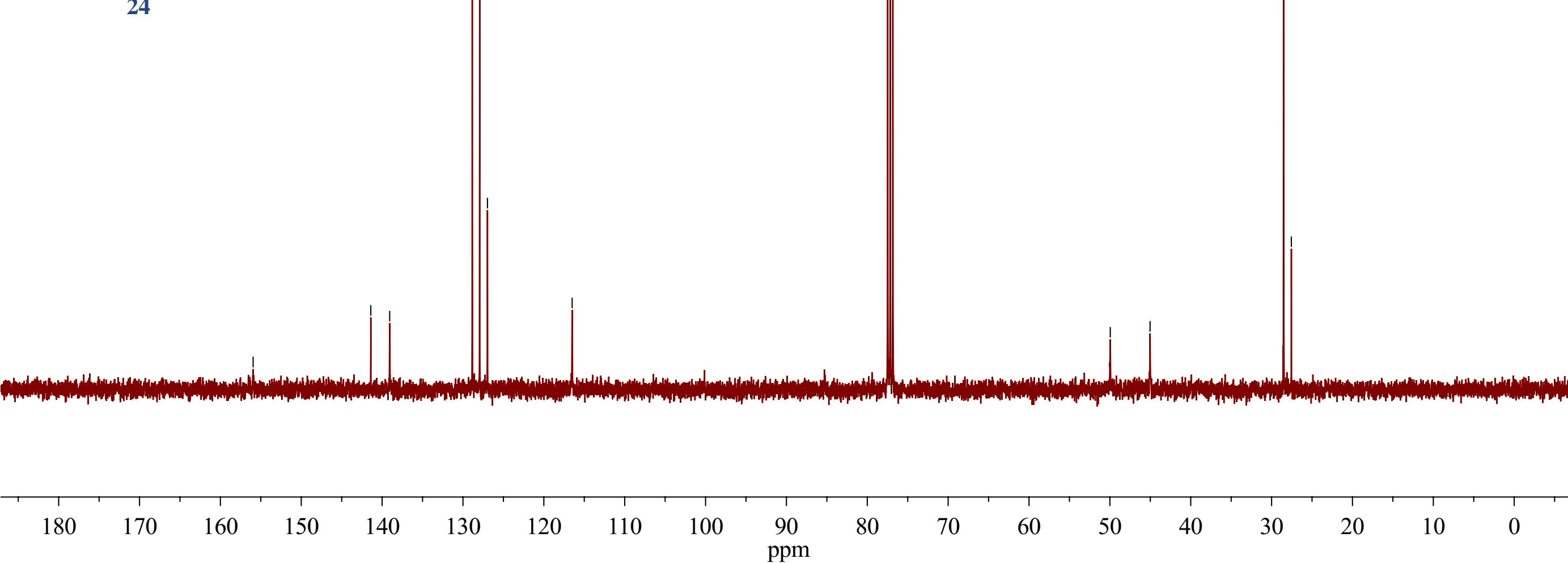

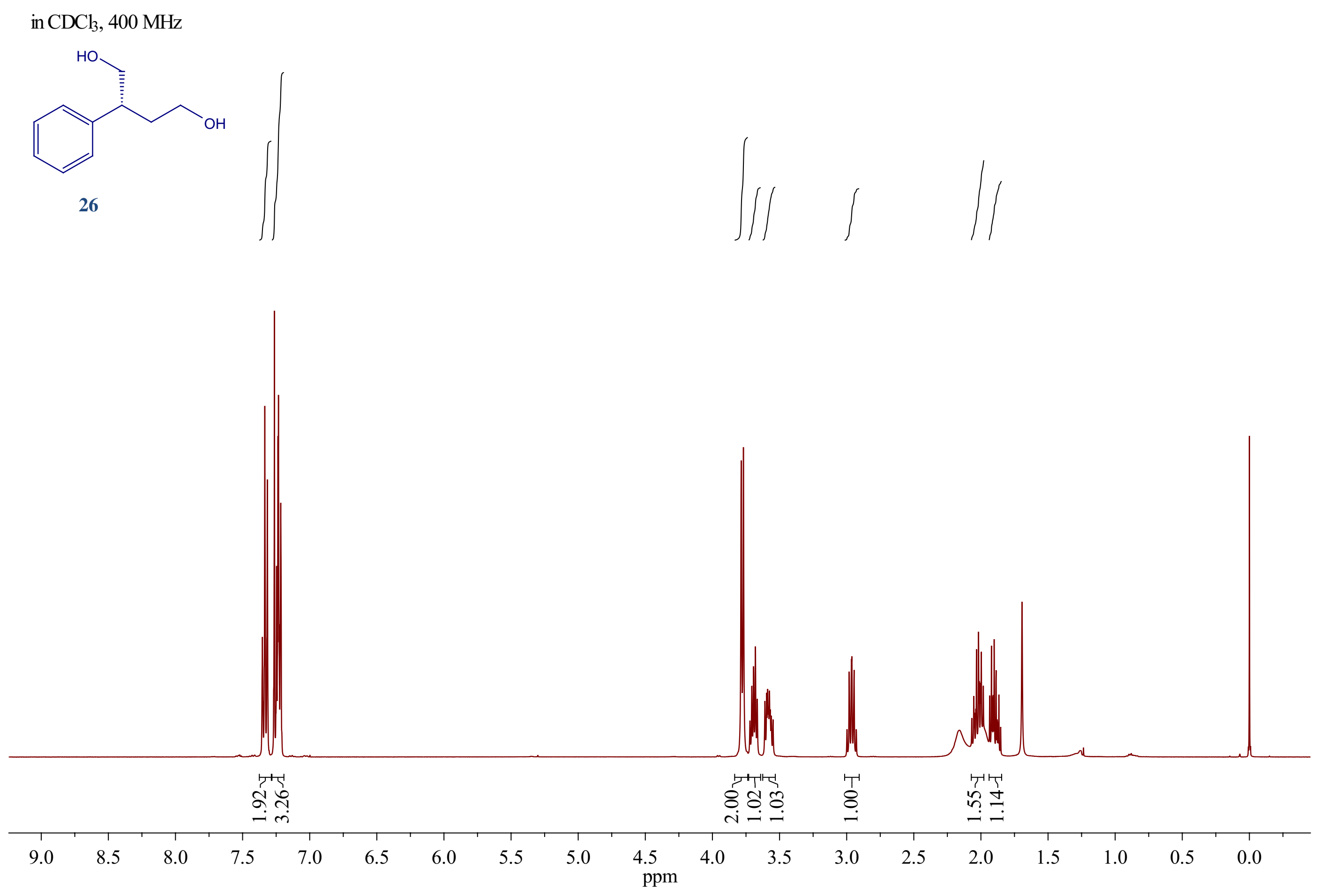


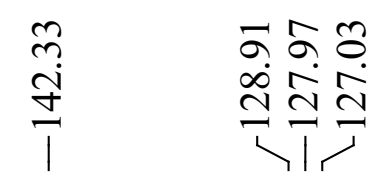

in $\mathrm{CDCl}_{3}, 101 \mathrm{MHz}$
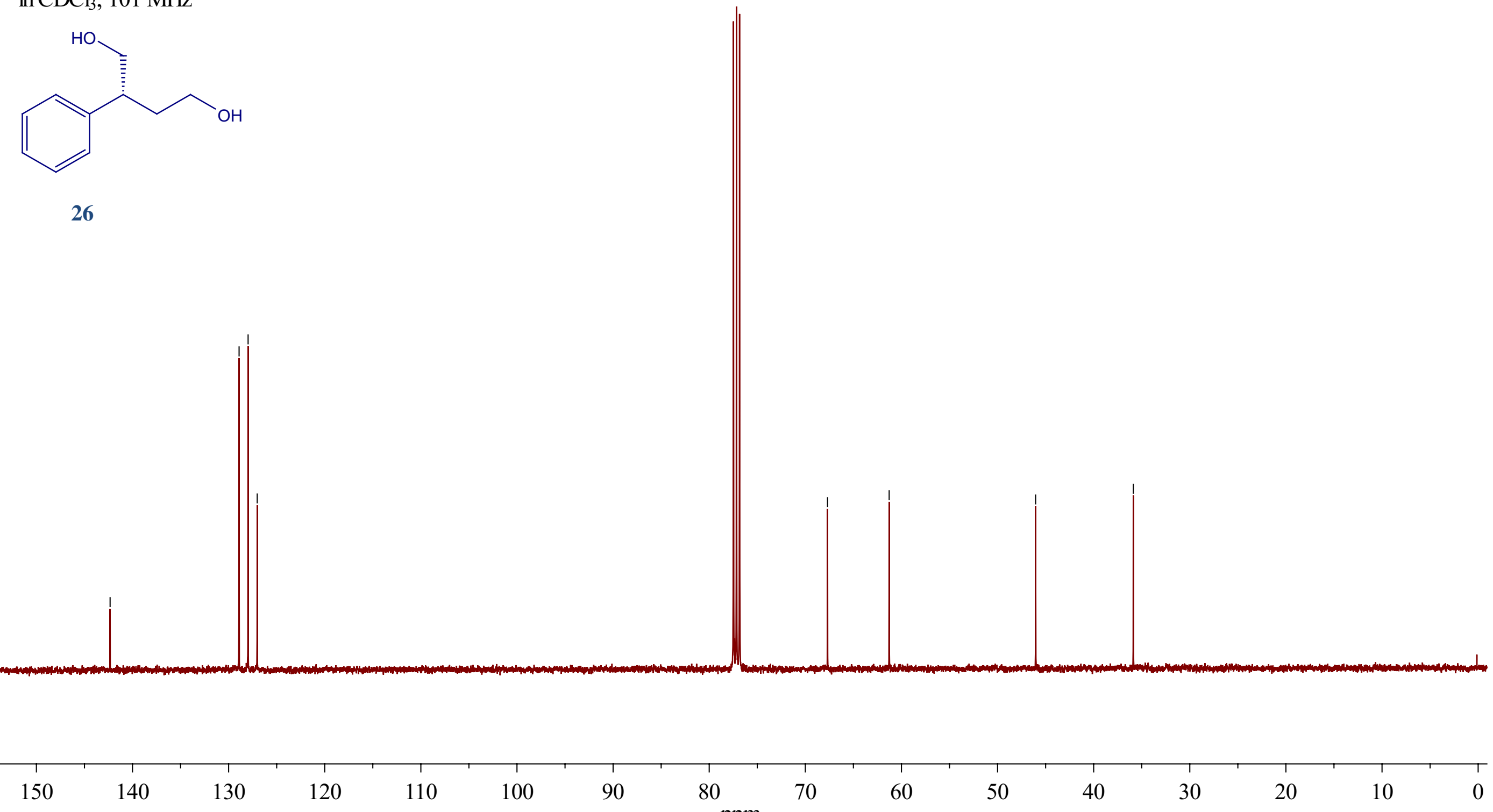


\section{References:}

1 W.-B. Liu, C. Zheng, C.-X. Zhuo, L.-X. Dai, S.-L. You, J. Am. Chem. Soc. 2012, 134, 4812; b) C. Defieber, M. A. Ariger, P. Moriel, E. M. Carreira, Angew. Chem. Int. Ed. 2007, 46, 3139; c) L. A Arnold, R. Imbos, A. Mandoli, A. H. M. de Vries, R. Naasz, B. L Feringa, Tetrahedron 2000, 55, 2865; d) W.-B. Liu, C. Zheng, C.X. Zhuo, L.-X. Dai, S.-L. You, J. Am. Chem. Soc. 2012, 134, 4812.

2 Z.-Q. Zhang, C.-T. Yang, L.-J. Liang, B. Xiao, X. Lu, J.-H. Liu, Y.-Y. Sun, T. B. Marder, Y. Fu, Org. Lett. 2014, 16, 6342.

3 D. J. Weix, D. Markovic, M. Ueda, J. F. Hartwig, Org. Lett. 2009, 11, 2944.

4 Q. Yuan, K. Yao, D. Liu, W. Zhang, Chem.Commun. 2015, 51, 11834.

5 P. Zhang, L. A. Brozek, J. P. Morken, J. Am. Chem. Soc. 2010,132, 10686.

6 L. M. Stanley, J. F. Hartwig, Angew. Chem. Int. Ed. 2009, 48, 7841.

7 B. M. Trost, N. G. Andersen, J. Am. Chem. Soc. 2002, 124, 14320.

8 Y. Sumida, S. Hayashi, K. Hirano, H. Yorimitsu, K. Oshima, Org. Lett. 2008, 10, 1629.

9 Y. Shi, A. H. Hoveyda, Angew. Chem. Int. Ed. 2016, 55, 3455.

10 a) T. Ito, L. E. Overman, J. Wang, J. Am. Chem. Soc. 2012, 134, 16449; b) Z. D. Aron, T. Ito, T. L. May, L. E. Overman, J. Wang, J. Org. Chem. 2013, 78, 9929.

11 S. N. Mlynarski, A. S. Karns, J. P. Morken, J. Am. Chem. Soc. 2012, 134, 16449.

12 Y.-C. Chung, D. Janmanchi, H.-L. Wu, Org. Lett. 2012, 14, 2766. 